\title{
Analysis of Internal Wave Induced Mode Coupling Effects on The 1995 SWARM Experiment Acoustic Transmissions
}

by

Robert Hugh Headrick

B.S. Chem. Eng., Oklahoma State University, 1983

M.S. Ocean Eng., M.I.T. / Woods Hole Oceanographic Institution, 1990

O.E. Ocean Eng., M.I.T. / Woods Hole Oceanographic Institution, 1990

Submitted in partial fulfillment of the

requirements for the degree of

DOCTOR OF PHILOSOPHY

at the

MASSACHUSETTS INSTITUTE OF TECHNOLOGY

and the

WOODS HOLE OCEANOGRAPHIC INSTITUTION

June 1997

(c) Robert Hugh Headrick, 1997. All rights reserved.

The author hereby grants to the United States Government, MIT, and WHOI

permission to reproduce and to distribute publicly paper and electronic copies of this thesis document in whole or in part.

Signature of Author .....

Joint Program in Applied Ocean Science and Engineering Massachusetts Institute of Technology/

- Woods Hole Oceanographic Institution

Certified by

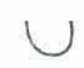

Dr. James F. Lynch

Associate Scientist, Woods Hole Oceanographic Institution Thesis Supervisor

Accepted by

Professor Henrik Schmidt

Chairman, Joint Committee for Applied Ocean Science and Engineering Massachusetts Institute of Technology-Woods Hole Oceanographic Institution

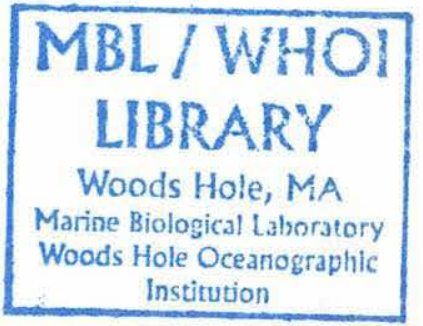




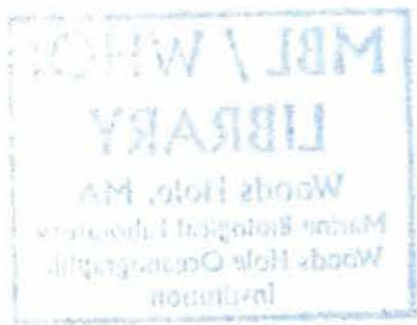




\title{
Analysis of Internal Wave Induced Mode Coupling Effects on The 1995 SWARM Experiment Acoustic Transmissions by

\author{
Robert Hugh Headrick
}

\author{
Submitted to the Massachusetts Institute of Technology/ \\ Woods Hole Oceanographic Institution \\ Joint Program in Oceanographic Engineering \\ on April 28, 1997 in partial fulfillment of the \\ Requirements for the Degree of Doctor of Philosophy in \\ Oceanographic Engineering
}

\begin{abstract}
As part of the Shallow Water Acoustics in a Random Medium (SWARM) experiment [1], a sixteen element WHOI vertical line array (WVLA) was moored in 70 meters of water off the New Jersey coast. This array was sampled at $1395 \mathrm{~Hz}$ or higher for the seven days it was deployed. Tomography sources with carrier frequencies of 224 and $400 \mathrm{~Hz}$ were moored about $32 \mathrm{~km}$ shoreward, such that the acoustic path was anti-parallel to the primary propagation direction for shelf generated internal wave solitons. Two models for the propagation of normal modes through a 2-D waveguide with solitary internal wave (soliton) scattering included are developed to help in understanding the very complicated mode arrivals seen at the WVLA. The simplest model uses the Preisig and Duda [2] sharp interface approximation for solitons, allowing for rapid analysis of the effects of various numbers of solitons on mode arrival statistics. The second model, using SWARM thermistor string data to simulate the actual SWARM waveguides, is more realistic, but much slower. The analysis of the actual WVLA data yields spread, bias, wander, and intensity fluctuation signals that are modulated at tidal frequencies. The signals are consistent with predicted relationships to the internal wave distributions in the waveguides.
\end{abstract}

Thesis Supervisor: Dr. James F. Lynch

Associate Scientist

Woods Hole Oceanographic Institution 


\section{Acknowledgments}

I am indebted to the United States Navy for giving me the opportunity to once again pursue graduate studies at MIT and WHOI. The funds for my education were provided by the Office of Naval Research through an ONR Fellowship (MIT award 002734-001); the funds for SWARM were also provided by the Office of Naval Research through ONR Grant N00014-95-0051.

I am very grateful to my thesis advisor Dr. James F. Lynch for his patience, guidance, and support. I thank him for allowing me to be a part of the SWARM experiment.

I would like to thank all the fellow SWARM conspirators, without whom much of this research would not have been possible. A particular thanks goes to Arthur Newhall; his knowledge of computers and close proximity (seated adjacent to me for over two years) were of immeasurable worth.

Thanks also goes to the rest of my thesis committee: Prof. Henrik Schmidt, Dr. Timothy Duda, and Dr. John Colosi. Their observations and comments were put to good use. Several "coupled-mode" discussions I had with Dr. Richard Evans were also very beneficial. I also thank Dr. Glen Gawarkiewicz for serving as Chairman of the Defense.

I thank my wife, Carolyn, and our three children, Bobby, Paul, and Sarah, for their enduring love and support.

Soli Deo Gloria 


\section{Contents}

1 Background $\quad 25$

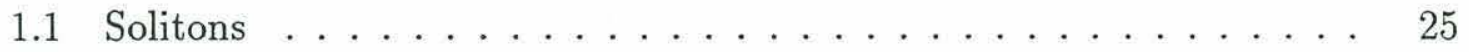

1.2 Acoustic Impact of Solitons . . . . . . . . . . . . . . . 28

1.2.1 Time Spreading . . . . . . . . . . . . . . . . 28

1.2.2 Attenuation . . . . . . . . . . . . . 30

1.3 Outline of Thesis ........................ 31

2 Data Collection $\quad 33$

2.1 Experiment Geometry . . . . . . . . . . . . . 33

2.2 Source Signals . . . . . . . . . . . . . . . . . . 33

2.3 Receiving Array . . . . . . . . . . . . . . . . . 34

2.4 Signal Isolation . . . . . . . . . . . . . . . 36

2.4 .1 Sequence Capture . . . . . . . . . . . . . . 36

2.4.2 Pulse Compression ................. 37

3 Mode Filtering $\quad 41$

3.1 Normal Modes. . . . . . . . . . . . . . . . . . . . . . 41

3.2 Array Tilt .......................... 44

3.3 Mode Filtering . . . . . . . . . . . . . . . . 45

3.3.1 Errors from Array Tilt . . . . . . . . . . . . 45

3.3.2 Time Dependence of Filter Performance . . . . . . . . . . . 47 
3.3.3 Mode Filter Outputs . . . . . . . . . . . . . . . . . 51

4 A Simple Scattering Model $\quad \mathbf{5 7}$

4.1 Normal Mode Pulse Propagation . . . . . . . . . . . . 57

4.1 .1 Total Field Summation . . . . . . . . . . . . 58

4.1 .2 Step Summation .................. 63

4.2 Incorporation of Field Measured SSPs . . . . . . . . . . . 65

4.2.1 Validity of $400 \mathrm{~Hz}$ Narrowband Models for a Broadband Pulse $\quad 67$

4.2.2 Adding Modal Attenuation . . . . . . . . . . . . 70

4.2.3 The Effects of Bottom Topography . . . . . . . . . . 73

4.3 Incorporation of a Background Internal Wave Spectrum . . . . . . . . 73

4.3.1 EOF Soundspeed Perturbation Modes . . . . . . . . . 75

4.4 Fine Scale Coupled Mode Computations . . . . . . . . . . 76

4.5 Putting it All Together . . . . . . . . . . . . . . 79

5 Model Analysis $\quad 81$

5.1 Monte Carlo Analysis of the SIA Model . . . . . . . . . . . . . 81

5.1.1 Relevant Statistics ................... 81

5.1.2 Simulation Parameters ................. 84

5.2 Analysis of the "Propagated Thermistor String Model" . . . . . . . . 102

5.2.1 An Example Waveguide . . . . . . . . . . . . . 102

5.2.2 Model Results . . . . . . . . . . . . . . . 102

5.3 The Transition to Real Data . . . . . . . . . . . . . 112

6 Analysis of Real Data $\quad 115$

$6.1400 \mathrm{~Hz}$ Mode 1 Arrival Analysis . . . . . . . . . . . . . 115

6.1 .1 Peak Arrival Time . . . . . . . . . . . . . 115

6.1 .2 Arrival Spread . . . . . . . . . . . . 121

6.1 .3 Peak Arrival Intensity . . . . . . . . . . . 126

6.1 .4 Higher Mode Statistics . . . . . . . . . . . . . 129 
$6.2224 \mathrm{~Hz}$ Arrival Analysis . . . . . . . . . . . . . . . . . . 133

6.2 .1 Peak Arrival Time . . . . . . . . . . . . . . . 133

$6.2 .2 \quad$ Spread Statistics . . . . . . . . . . . . . . . 139

6.2.3 Utility of Further $224 \mathrm{~Hz}$ Analysis . . . . . . . . . . . . 142

7 Conclusions 143

7.1 Conclusions . . . . . . . . . . . . . . . . . . . . 143

7.1 .1 Modeling . . . . . . . . . . . . . . . . . . . 143

7.1 .2 Data Analysis . . . . . . . . . . . . . . . . 145

7.2 Proposed Directions of Future Investigation $\ldots \ldots \ldots \ldots$

7.2 .1 Modeling . . . . . . . . . . . . . . . . . 146

7.2 .2 Data Analysis . . . . . . . . . . . . . . . . 146

7.2 .3 Experiments . . . . . . . . . . . . . . 146

7.3 Contributions . . . . . . . . . . . . . . . . . . 147

7.3 .1 Modeling . . . . . . . . . . . . . . . 147

7.3 .2 Data Analysis . . . . . . . . . . . . . . . . . 148

$\begin{array}{ll}\text { A Array Navigation } & 149\end{array}$

A.1 Navigation Set-Up . . . . . . . . . . . . . . . . . . . . 149

A.2 Expected Motions of the Array . . . . . . . . . . . . . . 149

A.3 Navigation Data Analysis . . . . . . . . . . . . . 153

A.3.1 Inversion for Mooring Motion . . . . . . . . . 153

A.3.2 Inversion with Temperature Correction Applied to Data . . 158

A.3.3 Inversion for Sound Speed Only . . . . . . . . . . . . 161

A.4 Conclusions . . . . . . . . . . . . . . . . . . . . 162

B Comparison of the Pulse Propagation Model with Pulse Synthesis using KRAKEN Complex Pressure Fields $\quad 167$

C Incorporation of Background Internal Wave Spectrum through Dozier 
and Tappert Coupling $\quad 173$

C.1 Coupled Mode Formulation . . . . . . . . . . . . . . . 173

C.1.1 EOF Sound-speed Perturbation Modes . . . . . . . . . . 175

C.1.2 Numerical Trials . . . . . . . . . . . . . . . . . . 178

C.2 Limitations of Dozier and Tappert Coupling . . . . . . . . . . . 180

C.2.1 Fine Scale Application . . . . . . . . . . . . . . . . . 182

$\begin{array}{ll}\text { D Modal Reciprocity } & 189\end{array}$ 


\section{List of Figures}

1-1 A simple two-layer model of an internal soliton, with the upper layer, $h_{1}=15 \mathrm{~m}$, lower layer, $h_{2}=55 \mathrm{~m}$, and reduced gravity, $g \Delta \rho / \rho=.01$ $\mathrm{m} / \mathrm{s}^{2}$. The $20 \mathrm{~m}$ depression propagates at a phase speed, $V=.8 \mathrm{~m} / \mathrm{s}$.

1-2 Time and space interpolation of four thermistor records. The thermistors were attached to the WVLA at water depths of $12.5,20.5,30.5$, and 40.5 meters and sampled at 30 second intervals. . . . . . . . .

1-3 Space transformation of the time series for the prominent soliton in the lower frame of Figure 1-2. All six available thermistors are used, so the depth spans 12.5 to 60.5 meters. The dimensions shown are properly scaled for soliton with a combined phase and advection speed of $.8 \mathrm{~m} / \mathrm{s} .29$

2-1 Source positions are noted by "*". The $400 \mathrm{~Hz}$ source is the shallower of the two. WVLA hydrophone positions are noted by "o" and the thermistors on the array are marked as "+". . . . . . . . . 34

2-2 Circular convolution of an ideal delayed $400 \mathrm{~Hz}$ transmitted sequence with an ideal non-delayed transmitted sequence. Both sequences have been zero-padded from length 7129 to length 8192 . The lower frame is a window of the full output above. . . . . . . . . . . . . 38

2-3 Typical $400 \mathrm{~Hz}$ arrivals at the WVLA hydrophones. The sets of 16 arrivals are separated by 6 minutes. . . . . . . . . . . . . . 39 
3-1 Mode rejection levels from mode-filter outputs, $A_{1}(*), A_{2}(\mathrm{x}), A_{3}(\mathrm{o})$, and $A_{4}(+)$, as a function of array tilt. The four plots represent tilted inputs of pure mode 1 , mode 2 , mode 3 , and mode 4 arrivals. These plots use mode filters and mode arrivals generated from the same typical SWARM SSP. . . . . . . . . . . . . . . . .

3-2 Mode rejection levels from mode-filter outputs, $A_{1}(*), A_{2}(\mathrm{x}), A_{3}(\mathrm{o})$, and $A_{4}(+)$, as a function of transmission number. The four plots are based on a series of untilted inputs of pure mode 1, mode 2, mode 3 , and mode 4 arrivals, where the mode filters and mode arrivals are generated from the same series of SSPs. . . . . . . . . . . .

3-3 Mode rejection levels from mode-filter outputs, $A_{1}(*), A_{2}(\mathrm{x}), A_{3}(\mathrm{o})$, and $A_{4}(+)$, as a function of transmission number. The four plots are based on 1 degree tilted inputs of pure mode 1 , mode 2 , mode 3 , and mode 4 arrivals, using mode filters and mode arrivals generated from the SSPs separated by 30 seconds. . . . . . . . . . . . . . .

3-4 Mode Coefficient outputs, $A_{1}$ (solid), $A_{2}$ (dashed), $A_{3}$ (dot-dash), and $A_{4}$ (dotted), as a function of time. The ten plots depict the arrival structure for the first of 22 captured sequences out of ten sequential

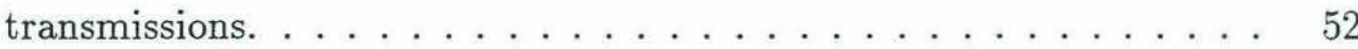

3-5 Mode coefficient outputs, $A_{1}$ (solid), $A_{2}$ (dashed), $A_{3}$ (dot-dash), and $A_{4}$ (dotted), as a function of time. The ten plots depict the arrival structure for every other sequence from the first to the 19th of 22 captured sequences from the time zero transmission in Figure 3-4. . . 54

3-6 Average correlation coefficients for outputs, $A_{1}$ (solid), $A_{2}$ (dashed), $A_{3}$ (dotdash), and $A_{4}$ (dotted), as a function of elapsed time. The average is taken over the ten transmissions of Figure $3-4 \ldots \ldots \ldots \ldots$ 
4-1 An illustration of the SIA approximation for solitons (left), and the idealized SSPs (right) used to model the interior and exterior regions of the SIA soliton. The dotted curve represents the exterior or background region, and the solid curve represents the interior. . . . . . . .

4-2 Propagation of four modes through $32 \mathrm{~km}$ of an idealized wave guide with one 200 meter soliton at the $20 \mathrm{~km}$ point. The solid curves are the absolute value of coherent sums of the $J^{2}=16$ dotted individually scattered arrivals. Starting amplitudes for mode 1 through 4 were 1.0, $0.8,0.6$, and 0.4 respectively. . . . . . . . . . . .

4-3 Propagation of four modes through $32 \mathrm{~km}$ of an idealized wave guide with 200 meter solitons at the 10 and $20 \mathrm{~km}$ points. The solid curves are the coherent sums of the $N^{2} \times N^{2}=256$ dotted individually scattered arrivals. Initial mode 1 through 4 amplitudes were 1.0, 0.8, 0.6, and 0.4 respectively. . . . . . . . . . . . . . . .

4-4 Simulated mode coefficient outputs, $A_{1}$ (solid), $A_{2}$ (dashed), $A_{3}$ (dotdash), and $A_{4}$ (dotted), for propagation through seven idealized solitons. The solitons propagated toward the receiver in lock step at 0.8 $\mathrm{m} / \mathrm{s}$. The ten plots depict the arrival structures for sequential realizations at six minute intervals. . . . . . . . . . . . . . . . 64

4-5 Time-series of temperature at a depth of 12.5 meters on the WVLA. .

4-6 Field measured SSPs for use in the pulse-propagation model. The dashed line is representative of a standard SSP with no soliton. The solid line represents the soliton SSP . . . . . . . . . . . . .

4-7 Mode shapes for field measured (SWARM) SSPs. The dashed lines are the $355 \mathrm{~Hz}$ (thin lines) and $445 \mathrm{~Hz}$ (thick lines) mode shapes corresponding to the standard SSP with no soliton. The solid lines are the same modes for the soliton SSP. The dotted line at $68 \mathrm{~m}$ indicates the sediment interface. . . . . . . . . . . . . . . 
4-8 Phase and group speeds for the standard field measured SSP. Mode 1 has the lowest phase speed and the highest group speed. The remaining phase speeds increase monotonically with mode number, and the group speeds decrease monotonically with mode number. . . . . . . . . . .

4-9 Effects of mode and frequency dispersion on single mode transmissions over $32 \mathrm{~km}$. The transmissions include no soliton scattering or intrinsic mode attenuation. The attenuation in the higher modes is due solely to frequency dispersion. . . . . . . . . . . . . .

4-10 Effects of mode and frequency dispersion combined with intrinsic mode attenuation on single mode transmissions over $10 \mathrm{~km}$. The transmissions include no soliton scattering. . . . . . . . . . . . . .

4-11 Mode coupling effects of the bottom topography shown in Figure 2-1 when the SSP is a modified version the "non-soliton" SSP of Figure 4-6. The "No Ducting" modification consists of imposing the $50.5 \mathrm{~m}$ temperature from $50.5 \mathrm{~m}$ to the bottom depth, and the "Ducting" modification consists of imposing the $60.5 \mathrm{~m}$ temperature from 50.5 $\mathrm{m}$ to the bottom depth. The thinner lines in this figure represent predicted arrival shapes for modes that propagate adiabatically. . . .

4-12 Comparison of fine scale coupled mode arrivals using $24 \mathrm{~m}$ (solid) and $48 \mathrm{~m}$ (dotted) step sizes. The $32 \mathrm{~km}$ wave guide under evaluation was generated from range propagated WVLA thermistor records. . . . . .

5-1 The mean (-), median $(\cdots)$, standard deviation $(-\cdot)$, and interquartile range (- -) of an unscattered mode 1 arrival plotted as a function of noise cutoff level. The median reaches its correct value of zero at a noise cutoff level of $-38 \mathrm{~dB}$ relative to the peak of the arrival. . . . . . 
5-2 The solid line shows the mode number space history of one of the $6^{20}$ subarrivals that would make up the mode 1 arrival from a six mode transmission through the 10 SIA soliton waveguide that is indicated by the dotted lines. . . . . . . . . . . . . . .

5-3 Random realizations of test waveguides with from 10 to $35200 \mathrm{~m}$ SIA solitons over $32 \mathrm{~km}$. The first soliton is placed randomly about $2.5 \mathrm{~km}$ from the source, and the remainder are added randomly from there, with an average of $450 \mathrm{~m}$ between leading edges. . . . . . . . . .

5-4 Single realizations of mode 1 (solid), mode 2 (dashed), mode 3 (dotdash), and mode 4 (dotted) arrivals for 0 to $35200 \mathrm{~m}$ SIA solitons over $32 \mathrm{~km}$. The simulated waveguides used are similar to those shown in Figure 5-3. The simulated source depth is $27 \mathrm{~m}$ in $68 \mathrm{~m}$ of water, and the compressional wave attenuation in the bottom is $.046 \mathrm{~dB} / \mathrm{m}$ at 400 Hz. Note: Mode 4 arrivals are not visible at this level of attenuation.

5-5 The same scenario as Figure 5-4, except the compressional wave attenuation in the bottom has been reduced from .046 to $0.0115 \mathrm{~dB} / \mathrm{m}$. . .

5-6 Histograms for peak position of mode 1 arrivals for 5 through 40 random solitons. The solitons start about $2.5 \mathrm{~km}$ from the source with an average separation of $450 \mathrm{~m}$ between soliton leading edges. Time zero corresponds to the PAM1 arrival. . . . . . . . . . . . .

5-7 The average mean (-), median (- $)$, and peak $(\cdots)$ lag with respect to the pseudo-adiabatic arrival, and standard deviation (-.) of scattered mode 1 arrivals plotted as a function of the number of solitons placed between the source and the receiver, where the solitons are added starting from the source end. The upper three lines are all bias measures, and the lower line is a measure of spread. . . . . . . . . . . 
5-8 The average mean $(-)$, median $(--)$, and peak $(\cdots)$ lag with respect to the pseudo-adiabatic arrival, and standard deviation (-.) of scattered mode 1 arrivals plotted as a function of the number of solitons placed between the source and the receiver. The upper curves, averages over 50 realizations, represent the case where the solitons are added starting at the receiver end. The lower set of curves is replotted from figure 5-5. 60 solitons is essentially saturation, and the same points are used in both sets of curves. . . . . . . . . . . . . . .

5-9 Time dependent waveguide with two sets of six SIA solitons. The upper frame shows the starting positions, the middle frame shows the positions five hours later, and the lower frame is plot of peak position bias (re PAM1) as a function of time. The solitons propagate in lockstep, traversing $48 \mathrm{~m}$ per minute, the time between each peak position data point (o). The mean arrival data is also in one minute increments, but the points are connected by a solid line. . . . . . . . . .

5-10 Waterfall of mode 1 arrival patterns from the time dependent waveguide with two sets of six SIA solitons. The 300 peak positions in the lower frame of Figure 5-9 were picked from these arrival patterns. . .

5-11 Snapshots of the arrival shapes for all eight modes propagated in the Figure 5-10 simulation. The left hand column shows the minute 1 arrival shapes and the right column shows the minute 300 arrival shapes. The thicker lines are the scattered arrival shapes, and the thinner lines show the corresponding unscattered mode arrivals. The eighth mode is only minimally excited by the source at $27 \mathrm{~m}$ depth, and it undergoes the most attenuation, so it is not even visible as an unscattered arrival. 98 
5-12 A comparison of mean (-) and peak (o) arrival time as function of the number of evenly spaced solitons in a $32 \mathrm{~km}$ waveguide. The first soliton is always $200 \mathrm{~m}$ from the source and the last soliton is roughly $32 \mathrm{~km}$ divided by the number of solitons away from the receiver. . .

5-13 The top graph shows $12.5 \mathrm{~m}$ depth temperature readings from the 2 hrs of thermistor data used to simulate modal excitation levels. The middle graph shows the resulting modal excitation levels for mode 1 (solid), mode 2 (dashed), mode 3 (dot-dash), and mode 4 (dotted). Mode 5 is similar in magnitude and used as an input to the model, but it is omitted from this figure to avoid clutter. The lower graph shows the output bias levels corresponding to the excitation inputs shown in the middle graph. The solid line is the mode 1 arrival envelope peak arrival time in excess of the PAM1 arrival time and the dotted line is the mode 1 arrival envelope mean arrival time in excess of the PAM1 arrival time. . . . . . . . . . . . . . . . . . . 10

5-14 Pycnocline displacements from an Orr backscatter record compared to a thermistor string record using a propagation speed of $.62 \mathrm{~m} / \mathrm{s}$ over the distance between the back scatter instrument and the thermistor string at each backscatter reading. . . . . . . . . . . . . . . 103

5-15 Distribution of peaks for the mode 1 arrivals of simulated transmissions using sequential WVLA thermistor string generated waveguides. The sequential 32160 by $68 \mathrm{~m}$ waveguides are separated by six minutes. The size of each arrival dot is scaled by the amplitude of the peak arrival. The upper frame includes a plot of the PAM1 arrival time in conjunction with the individual peak arrival times; the zero reference on the arrival time axis corresponds to 21.6 seconds. The lower frame plots the bias or offset of the peak arrivals relative to the PAM1 arrival time for each realization. . . . . . . . . . . . . . . . . . 
5-16 Distribution of peaks for the mode 2 arrivals corresponding to the mode 1 arrivals of Figure 5-15. The upper frame includes a plot of both the PAM1 arrival time (dotted line) and PAM2 arrival time (solid line), in conjunction with the individual peak arrival times; the zero reference on the arrival time axis corresponds to 21.6 seconds. The lower frame plots the bias or offset of the mode 2 peak arrivals relative to the PAM2 arrival time for each realization. . . . . . . . . . . . . . 106

5-17 30 minute bin averaged IQR measurements and the WVLA temperature profile for the simulation test period. The profiled temperatures range from 7.2 to 25.9 degrees $\mathrm{C}$. The top frame is for mode 1 and the bottom is for mode2. . . . . . . . . . . . . . . . . . . 108

5-18 A comparison of 1 hour bin averaged IQR levels for the simulated mode 2-4 data (solid lines) and a $1 \mathrm{hr}$ bin average of the Standard Deviation of the Temperature at $22.5 \mathrm{~m}$ depth over the previous 4.3 hrs (dashed lines). The standard deviation of the temperature is in tenths of a degree centigrade to facilitate comparison with the IQR levels in milliseconds. . . . . . . . . . . . . . . . . . . . . . 110

5-19 Cross-correlations of $1.4 \mathrm{hr}$ averaged peak height fluctuations for mode 1 (solid), 2 (dashed), 3 (dot-dashed), and 4 (dotted) with the corresponding $1.4 \mathrm{hr}$ averaged mode $1 \mathrm{IQR}$ (spread) levels. . . . . . . . .

6-1 Distribution of SWARM Mode 1 Peak arrival times. The zero reference is at an arrival time of 21.985 seconds. The plotted transmissions occurred every six minutes, with each transmission producing 22 sequence arrivals over a two minute period. The location of the highest peak in each sequence is plotted. Though not evident in most cases, there are gaps in the record that range from single lost transmissions to occasional larger gaps of up to two hours in duration. 
6-2 Predicted current effects on PAM1 arrival time plotted with the distribution of peak arrival times shown in Figure 6-1. The solid curve is based on the mean current between 39 and $59 \mathrm{~m}$ depth and the dotted curve is based on the average current between 11 and $67 \mathrm{~m}$ depth. . . 118

6-3 Predicted temperature fluctuation effects on PAM1 arrival time (solid curve) plotted with the distribution of "current-corrected" peak arrival times. The solid curve is zero mean (by definition) and offset from the data for convenience. . . . . . . . . . . . . . . . . 11

6-4 Mode 1 peak arrival time bias histograms for the data shown in Figure 6-1 and the model distribution shown Figure 5-15. The bias measurements are relative to estimated PAM1 arrival times (i.e. the leading edge) for the data and calculated PAM1 arrival times for the model. .

6-5 Mode 1 arrival spread, as measured by the IQR of the arrival envelope (unscattered IQR is about $7.2 \mathrm{~ms}$ ). The upper frame is plot of the spread when averaged over each transmission (22 sequences per transmission). The lower frame shows bin averaged spreads for five bin widths ranging from 1.4 to $4.1 \mathrm{hrs} . \ldots \ldots \ldots \ldots \ldots$

6-6 Power Spectral Density of the bin averaged spreads shown in the lower frame of Figure 6-5 . . . . . . . . . . . . . . . . . . . 124

6-7 WVLA temperature as function of time and depth overlaid by the 1.4 hr bin averaged IQR of mode 1 arrivals at the WVLA. The temperatures in this figure range from 7.2 to 26.5 degrees C. . . . . . . . 125

6-8 A comparison of 1 hour bin averaged IQR levels for the SWARM data (solid line), the corresponding propagated thermistor string model (dotted line), and the Standard Deviation of the Temperature at $22.5 \mathrm{~m}$ depth over the previous $4.3 \mathrm{hrs}$ (dashed line). The standard deviation of the temperature is in tenths of a degree $\mathrm{C}$ to facilitate comparison with the IQR levels in milliseconds. . . . . . . . . . . . . . 127 
6-9 A comparison of $1.4 \mathrm{hr}$ averaged peak intensity fluctuations and the corresponding $1.4 \mathrm{hr}$ averaged IQR levels for the SWARM data. The peak intensity fluctuations are plotted in terms of relative loss to provide a signal that is in phase with the spread fluctuations. . . . . . . 128

6-10 A comparison of $1.4 \mathrm{hr}$ averaged peak-height fluctuations for the first four modes of the SWARM data. Mode 1 is solid, mode 2 is dot-dashed, mode 3 is dashed, and mode 4 is dotted. . . . . . . . . . . . . . . 130

6-11 Cross-correlations of $1.4 \mathrm{hr}$ averaged peak height fluctuations for mode 1 (solid), 2 (dashed), 3 (dot-dashed), and 4 (dotted) with the corresponding $1.4 \mathrm{hr}$ averaged mode $1 \mathrm{IQR}$ (spread) levels for the SWARM

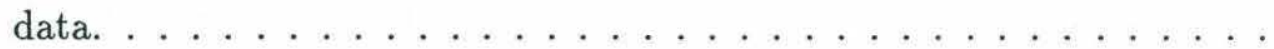

6-12 Mode 1 (solid), mode 2 (dashed), mode 3 (dot-dashed), and Mode 4 (dotted) peak arrival time leading edge (connects earliest peak arrivals in $1.1 \mathrm{hr}$ bins) and $1.4 \mathrm{hr}$ bin average mean peak arrival times. The leading edges for modes 2-4 are bracketed between the two mode 1 solid lines, and the mean arrivals for modes 2-4 are positioned above the upper solid line. . . . . . . . . . . . . . . . .

6-13 Distribution of SWARM $224 \mathrm{~Hz}$ Mode 1 Peak arrival times. The zero reference is at an arrival time of 22.453 seconds. The plotted transmissions occurred every five minutes. Only 8 sequences (every 4 th from 1 to 29$)$ are shown of the 29 available. An upshifted leading edge of the $400 \mathrm{~Hz}$ peak arrival distribution is also shown for comparison. The later zero reference time, relative to Figure 6-1, stems from the difference in source mooring locations. . . . . . . . . . . . . 135

6-14 Average arrival time difference between the $224 \mathrm{~Hz}$ PAM1, PAM2, and PAM3 arrivals and the $400 \mathrm{~Hz}$ PAM1 arrival. The arrival times are based on 12 hour average SSPs at the WVLA employed in a range independent fashion over a distance of $32 \mathrm{~km}$. 
6-15 $224 \mathrm{~Hz} 1.4 \mathrm{hr}$ bin averaged peak arrival times and peak heights. Mode 1 is solid, mode 2 is dashed, and mode 3 is dotted. . . . . . . . . . 139

6-16 Power spectral densities for the $224 \mathrm{~Hz} 1.4 \mathrm{hr}$ bin averaged peak arrival time and peak height time series of Figure 6-15. Mode 1 is solid, mode 2 is dashed, and mode 3 is dotted. . . . . . . . . . . . . . . . . 140

6-17 Comparison of $224 \mathrm{~Hz}$ and $400 \mathrm{~Hz} 1.4 \mathrm{hr}$ bin averaged IQR (spread). The IQR of an unscattered $224 \mathrm{~Hz}$ pulse would be about $37 \mathrm{~ms}(7 \mathrm{~ms}$ for a $400 \mathrm{~Hz}$ pulse) . . . . . . . . . . . . . . . .

A-1 Relative positions of transponder balls around the WHOI Vertical Line Array (WVLA). Transponders were interrogated every four minutes by a pinger at the base of the array. Round trip travel-time to the top hydrophone of the array was recorded. . . . . . . . . . 150

A-2 A simple form drag model for deflection of the WVLA where the drag force on the cable is all applied at the midpoint. The $20 \mathrm{~cm} / \mathrm{s}$ current shown would create a displacement of about $.5 \mathrm{~m}$ at the top hydrophone.151

A-3 The first (..) and fourteenth (-) depth bins of a 16 channel bottommounted acoustic Doppler current meter (ADCP) moored about $3 \mathrm{~km}$ from the WVLA. . . . . . . . . . . . . . . . . . . . 154

A-4 Travel-time fluctuation data-vectors associated with the three transponder ball paths used to navigate the WVLA . . . . . . . . . 155

A-5 Model vectors of an over-determined least-squares inversion of the navigation data for north and east displacement of the hydrophone array. 156

A-6 Model vectors of a least-squares inversion of the navigation data for north displacement, east displacement, and an average deviation in sound speed common to all three transponder return path integrals. . 159

A-7 A least-squares inversion for sound speed deviation produces fluctuations similar those observed in a 12.5 meter depth thermistor record that has been converted to sound speed. . . . . . . . . . 160 
A-8 A least-squares inversion for sound speed deviation produces fluctuations similar those observed in a 12.5 meter depth thermistor record that has been converted to sound speed. . . . . . . . . . . . 163

A-9 A least-squares inversion for sound speed deviations yields an 11.0/11.5 $\mathrm{kHz}$ path sound speed fluctuation (solid line) that is very similar those observed in a 12.5 meter depth thermistor record (dashed line). . . . 164

A-10 $12.0 \mathrm{kHz}$ path travel-time fluctuations (upper line) are very similar those calculated by taking the range to the transponder and back divided the average of the WVLA soundspeeds at 40, 50, and 60 meters

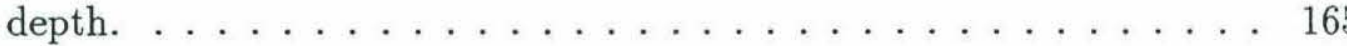

B-1 A comparison of single hydrophone pulse arrivals computed from the PPM (solid) and KFS (dotted) methods. The modeled waveguide uses the SWARM SSPs shown in Figure 4-6, with coupling from one SSP to the other occurring at 13,000 and $18,000 \mathrm{~m}$. The source depth is 27 $\mathrm{m}$ and the receiver depth at all five ranges is $50 \mathrm{~m} \ldots \ldots \ldots \ldots$

B-2 An eight soliton waveguide (upper panel) and two corresponding arrivals computed from the PPM (solid) and KFS (dotted) methods. The modeled waveguide uses the SWARM SSPs shown in Figure 4-6 to simulate the solitons. The source $\left(^{*}\right)$ depth is $27 \mathrm{~m}$ and the receiver (o) depth at both ranges is $50 \mathrm{~m} \ldots \ldots \ldots \ldots \ldots \ldots$

B-3 Single hydrophone RIP pulse arrivals computed from the PPM (solid) and KFS (dotted) methods using various source depths with the receiver depth fixed at $50 \mathrm{~m} . \ldots \ldots \ldots \ldots 171$ 
C-1 Effects of linear internal wave scattering on mode propagation. The results shown are the average of 50 trials using a sound-speed perturbation model with a range and depth averaged $\left\langle\left(\delta c / c_{0}\right)^{2}\right\rangle$ of $1.4 \times 10^{-5}$. The mode shapes used in the coupling equations were from the standard field measured SSP at $400 \mathrm{~Hz}$. . . . . . . . . . . . 179

C-2 Asymptotic behavior of $S I+1$ for mode 1 . The plotted quantity decreases to the asymptotic value of the growth rate factor $\chi$. The mode shapes and attenuation parameters used in the coupling equations were from the standard field measured SSP at $400 \mathrm{~Hz} . \ldots \ldots$. . . . 18

C-3 Mean sound speed profiles obtained from the WVLA thermistor records. The solid line is an average over yearday 211-217, and the dashed line is averaged over yearday 211 only. The dotted SSP is taken from early in yearday 211 when no solitons were at the WVLA. All three are extrapolated for depths above $12.5 \mathrm{~m}$ and below $60.5 \mathrm{~m} . \ldots \ldots 183$

C-4 Magnitudes of discrete coupling matrix coefficients using a $48 \mathrm{~m}$ step size with three different background profiles. The solid lines are computed using the yearday 211-217 mean profile, the dashed lines are from the yearday 211 mean profile, and the dotted lines are computed using the yearday 211 "no soliton" SSP. . . . . . . . . . . . . 184

C-5 Predictions of mode 1 arrivals made with three different background profiles. The solid lines are computed using the yearday 211-217 mean profile, the dashed lines are from the yearday 211 mean profile, and the dotted lines are computed using the yearday 211 "no soliton" SSP. 185

C-6 Twelve hours of predicted mode 1 arrivals using the yearday 211 mean profile and the yearday 211-217 mean profile. . . . . . . . . 186 
D-1 A comparison of reciprocal transmissions through an asymmetric SIA soliton waveguide (upper frame). Mode 1 through 4 arrivals as well the time domain pulse at a single transducer $(27 \mathrm{~m})$ are shown for both the forward and reciprocal transmissions. The source/receiver depth is $27 \mathrm{~m}$ for both transducers. . . . . . . . . . . . . 190

D-2 A comparison of reciprocal transmissions through a strong front (upper frame). Mode 1 through 4 arrivals as well the time domain pulse at the receiver are shown for both the forward and reciprocal transmissions. The source/receiver depth is $50 \mathrm{~m}$ at $0 \mathrm{~km}$ and $27 \mathrm{~m}$ at $32 \mathrm{~km}$. . . . 191 


\section{List of Tables}

2.1 Data set outline for the WVLA. Tapes 1 through 8 contain telemetered data that is sometimes broken up when the ship was out of range. Tapes 9 and 10 contain internally recorded data that fills in most of the gaps in the telemetered data. . . . . . . . . . . . .

4.1 Intrinsic mode attenuation parameters, $\gamma$, for various mode numbers. Values for both a soliton SSP and the standard field measured SSP without a soliton present are given. . . . . . . . . . . . .

5.1 Subarrival statistics for the simple two mode $50 / 50$ scattering scenario where there are four equally spaced increments and the difference between the PAM1 and PAM2 arrival times is $\Delta T=1 \ldots \ldots \ldots 94$

5.2 Zero-lag cross correlations between 1.4 hour bin averaged mode 1-4 peak heights and mode 1-4 mean spread levels. . . . . . . . . . . 112

6.1 Zero-lag cross correlations of 1.4 hour bin averaged peak heights for the SWARM data and the simulated data of Chapter 5. The unmixed data (modes 1 and 2) are shown in Figures 5-14 and 5-15; the cross-talk contaminated is from the same source, with incoherent mixing based on the "Average" filter performance matrix shown in section 3.3.2. . . 
6.2 Zero-lag cross correlations between 1.4 hour bin averaged mode 1-4 peak heights and mode 1-4 mean spread levels. The spread measurement employed for these cross-correlations is the standard deviation of peak arrival times over a transmission. . . . . . . . . . . . . 132

6.3 Hypothetical range independent travel times corresponding to daily averaged SSPs at the WVLA. The travel time differences between 224 $\mathrm{Hz}$ and $400 \mathrm{~Hz}$ modes are also listed. . . . . . . . 136 


\section{Chapter 1}

\section{Background}

The Shallow Water Acoustics in a Random Medium (SWARM) experiment [1], conducted off the coast of New Jersey near the continental shelfbreak in July and August of 1995, was dedicated to furthering our understanding of acoustic mode scattering effects of both linear internal waves and nonlinear solitary internal waves (solitons) in shallow water.

\subsection{Solitons}

A stratified ocean will support a variety of internal waves, the most common being the internal equivalent of a linear surface gravity wave. In the deep ocean, these waves are generally isotropic in direction and have frequency/vertical mode power spectra that are red and dominated by the first few modes. The canonical Garrett-Munk (GM) spectrum [3] normally provides a good model for the observed power spectra in deep water. The story is quite different along the United States East Coast continental shelf and other shallow regions, where the linear waves are non-isotropic and the internal wave field can also be heavily influenced by nonlinear solitary waves.

Internal solitons appear to be generated in groups or packets at the continental shelfbreak when the tide shifts from an ebb to a flood. Each "developed" packet usu- 
ally contains six to twelve solitons that are large enough to have a surface expression on radar, so the term "solitary wave" is something of a misnomer in this case. An account of the first observation of a surface solitary wave, made by John Scott Russell in 1834, is provided by Strang [4]:

I was observing the motion of a boat which was rapidly drawn along a narrow channel by a pair of horses, when the boat suddenly stopped... not so the mass of water in the channel which it had put in motion; it accumulated round the prow of the vessel in a state of violent agitation, then suddenly leaving it behind, rolled forward with great velocity, assuming the form of a large solitary elevation, a rounded, smooth and well-defined heap of water, which continued its course along the channel apparently without change of form or diminution of speed. I followed it on horseback, and overtook it still rolling at a rate of some eight to ten miles an hour, preserving its original figure some thirty feet long and a foot to a foot and a half in height. Its height gradually diminished, and after a chase of one or two miles I lost it in the windings of the channel.

This surface soliton can be modeled by the Korteweg-de Vries (KdV) equation,

$$
\eta_{t}+6 \eta \eta_{x}+\eta_{x x x}=0
$$

where the wave solution,

$$
\eta(x-c t)=\frac{c}{2} \operatorname{sech}^{2}\left(\frac{1}{2} \sqrt{c}(x-c t)\right)
$$

has a single hump whose height, $\frac{1}{2} c$, is proportional to the speed at which it travels to the right [4].

In contrast to the surface soliton's hump, internal solitons normally appear as a $\operatorname{sech}^{2}$ shaped depression in the pycnocline. In covering some theoretical aspects of internal solitons, Apel et al. [5] provide a solution for the following internal wave 
KdV equation,

$$
\eta_{t}+c \eta_{x}+\alpha \eta \eta_{x}+\beta \eta_{x x x}=0
$$

namely,

$$
\eta(x, t)=\eta_{0} \operatorname{sech}^{2}\left(\frac{x-V t}{\Delta}\right),
$$

where the soliton phase speed, $V$, is,

$$
V=c+\alpha \eta_{0} / 3
$$

and the characteristic width, $\Delta$, is given by,

$$
\Delta^{2}=\frac{12 \beta}{\alpha \eta_{0}}
$$

For a simple two-layer ocean density model, the constants are

$$
\begin{aligned}
c & =\left[\frac{g\left(\rho_{2}-\rho_{1}\right) h_{1} h_{2}}{\rho_{2} h_{1}+\rho_{1} h_{2}}\right]^{\frac{1}{2}} \\
\alpha & =\frac{3 c}{2 h_{1} h_{2}}\left(\frac{\rho_{2} h_{2}^{2}-\rho_{1} h_{2}^{2}}{\rho_{2} h_{1}+\rho_{1} h_{2}}\right) \\
\beta & =\frac{c h_{1} h_{2}}{6}\left(\frac{\rho_{1} h_{1}+\rho_{2} h_{2}}{\rho_{2} h_{1}+\rho_{1} h_{2}}\right) .
\end{aligned}
$$

Where $h_{1}$ and $h_{2}$ are the thicknesses of the upper and lower layers, and $\rho_{1}$ and $\rho_{2}$ are the respective densities. From the characteristic width formulation, we see that $\eta_{0}$ will have the same sign as $\alpha$, so for the most common case of a shallow pycnocline over a deeper layer (i.e. $\rho_{2} h_{1}^{2}<\rho_{1} h_{2}^{2}$ ), $\eta$ will be negative (i.e. downgoing). Employing this model with reasonable 2-layer approximations for a large SWARM soliton, $\eta_{0}=20$ $\mathrm{m}, h_{1}=15 \mathrm{~m}, h_{2}=55 \mathrm{~m}$, and $g \Delta \rho / \rho=.01 \mathrm{~m} / \mathrm{s}^{2}$, we predict a rather typical soliton phase speed of $V=.8 \mathrm{~m} / \mathrm{s}$ and the wave shape shown in Figure 1-1. The linear phase speed, $c$, for this case is $0.34 \mathrm{~m} / \mathrm{s}$ for comparison.

The internal solitary wave packets are often so numerous and closely spaced on the New Jersey shelf in summer that a nearly continuous train of solitons can pass a fixed instrument over the course of several hours. This is illustrated in Figure 1-2, where an 


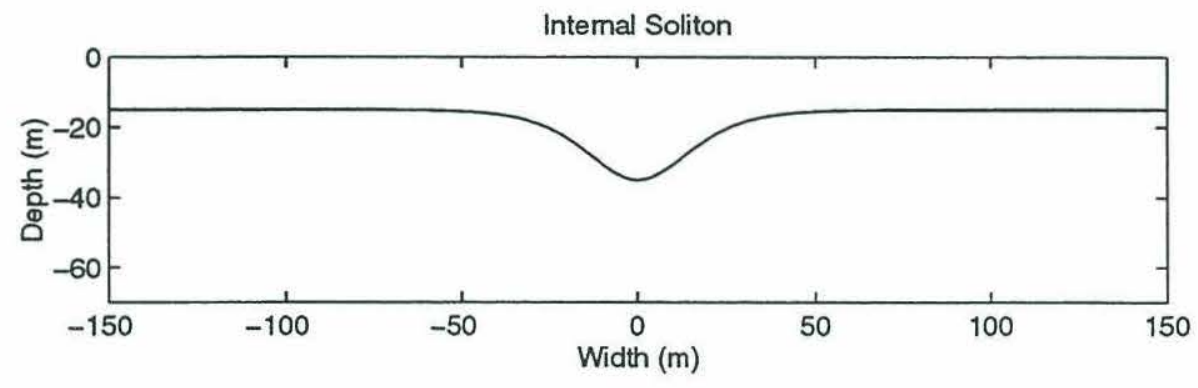

Figure 1-1: A simple two-layer model of an internal soliton, with the upper layer, $h_{1}=15 \mathrm{~m}$, lower layer, $h_{2}=55 \mathrm{~m}$, and reduced gravity, $g \Delta \rho / \rho=.01 \mathrm{~m} / \mathrm{s}^{2}$. The 20 $\mathrm{m}$ depression propagates at a phase speed, $V=.8 \mathrm{~m} / \mathrm{s}$.

array of thermistors attached to the WHOI Vertical Line Array (WVLA) records the passage of several packets over a six hour period. The mixture of time and space in this figure creates a distorted view of the soliton shapes, but this can be approximately corrected by applying a constant phase speed to a selected soliton, thus transforming the time coordinate into a space coordinate. This has been accomplished in Figure 1-3, where the time series for the prominent soliton in the lower frame of Figure 1-2 has been transformed into a properly scaled space representation.

\subsection{Acoustic Impact of Solitons}

The first investigation of the acoustic impact of solitons was reported by Zhou et al. [6] in 1991. This paper generated interest in the subject, and further strides have been made in the several investigations that have followed $[1,2,7,8,9]$.

\subsubsection{Time Spreading}

Internal solitons often have first mode displacements at the pycnocline ranging from 10 to 20 meters. These large displacements occur over relatively short wavelengths (Figure 1-3), creating strong horizontal gradients in soundspeed, which thus produces coupling between acoustic normal modes. Differences in the acoustic modal group velocities cause time spreading of pulses as energy is transferred between different 

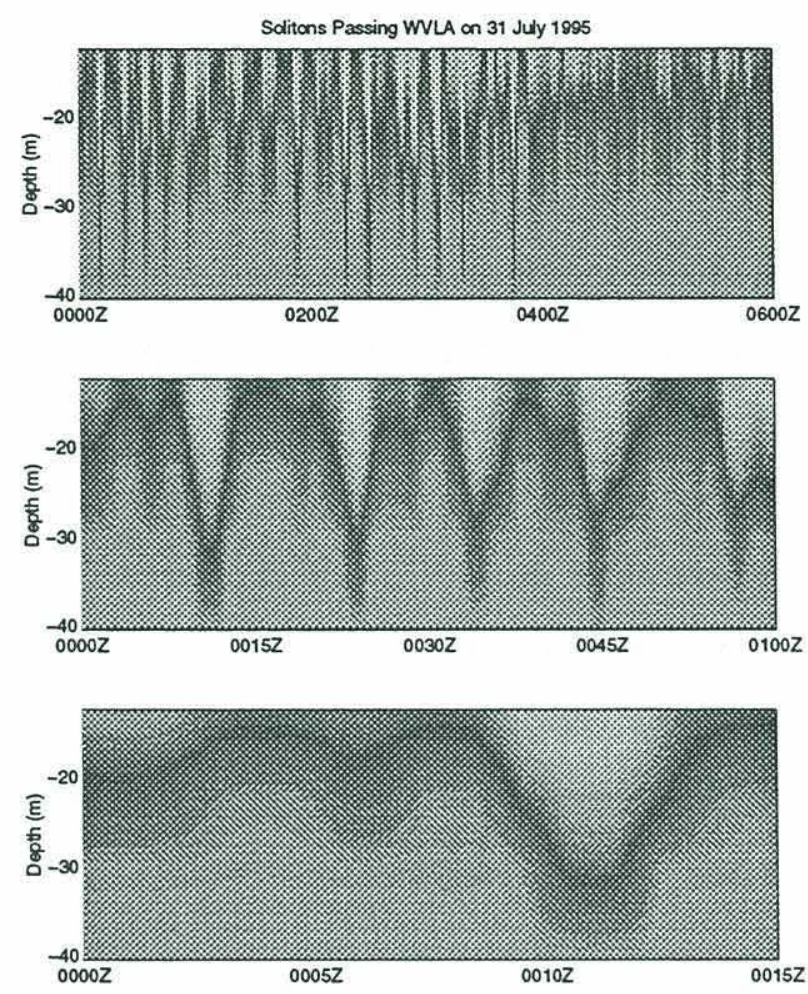

Figure 1-2: Time and space interpolation of four thermistor records. The thermistors were attached to the WVLA at water depths of $12.5,20.5,30.5$, and 40.5 meters and sampled at 30 second intervals.

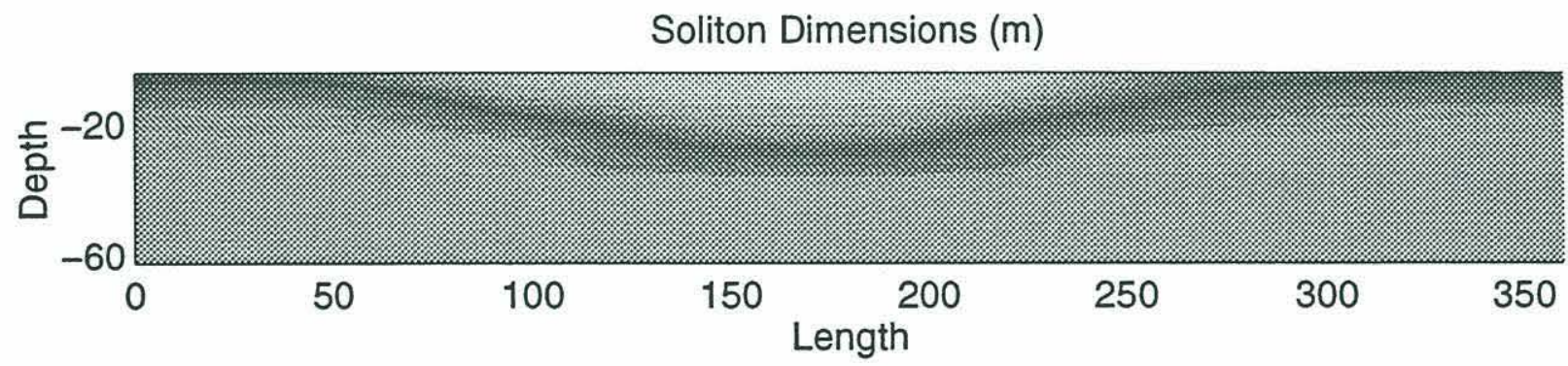

Figure 1-3: Space transformation of the time series for the prominent soliton in the lower frame of Figure 1-2. All six available thermistors are used, so the depth spans 12.5 to 60.5 meters. The dimensions shown are properly scaled for soliton with a combined phase and advection speed of $.8 \mathrm{~m} / \mathrm{s}$. 
modes. In the SWARM waveguide, mode 1 has the highest group speed, so the energy that transits in other modes for a portion of the trip is delayed with respect to energy that traveled exclusively in mode 1 . This causes the spread of mode 1 energy to be all in one direction (i.e. there is a positive bias in the mean and median traveltime of mode 1 energy relative to an unscattered mode 1 arrival time). Higher modes may have some energy coming in earlier (from traveling partially in lower modes) and some coming in later (from traveling partially in higher modes), so there may be negative, positive, or even zero bias in travel-time for these modes. As shown by Colosi and Flatté [10] the coupling caused by linear internal waves is similar, but reversed, in deep water where mode 1 generally has the lowest group speed.

Repeated coupling processes create mode arrivals that are sums of numerous "mode multipaths" that propagate through the range and wavenumber (mode number) space between the source and the receiver. Constructive and destructive interference between "mode multipaths" creates peaks and nulls in the arrival pattern. The detailed interference patterns are very sensitive to the spacing between the coupling events. Thus, given the tidal period variations in the size and numbers of solitons between the source and receiver and the continuous variations in soliton position and spacing (caused by their differing phase speeds), significant temporal variations in arrival-time patterns are to be expected.

\subsubsection{Attenuation}

Another effect of the coupling of mode 1 into higher modes is the apparent increased attenuation of mode 1 discussed by Zhou et al. [6]. In general modal attenuation is greater for higher mode numbers, so coupling is an energy sink for low modes and a source for higher modes. This mode-stripping along with long-term variations in the amount of coupling and short-term variations in interference patterns creates significant variability in the apparent attenuation of mode 1 energy. 


\subsection{Outline of Thesis}

Chapter 2 discusses SWARM data that are relevant to this thesis, along with other details such as experiment geometry, signal characteristics, data storage requirements, and pulse-compression schemes. Several issues associated with mode-filtering are then addressed in Chapter 3: a basic theory of normal mode propagation; analysis of possible errors in the mode filters used; and the accuracy of the filter outputs relative to the actual mode arrivals.

A model for the propagation of normal modes through a 2-D waveguide with solitons is developed in Chapter 4. Chapter 5 discusses pulse propagation simulations using two different forms of the model. The simplest form of the model, which uses Preisig and Duda's [2] sharp interface approximation (an inverted square-wave) for solitons, was tested by varying the numbers and locations of solitons and the amount of bottom attenuation. In observing the sensitivity of mode arrival statistics to changes in the model parameters, candidate statistics for quantifying the spread and bias of mode arrivals were evaluated. The second, more complicated, form of the model is based on range propagated thermistor string records, so it models the effects of both linear and nonlinear internal waves. It is used to try to model the temporal variations in the SWARM waveguide during the time of the actual experiment.

Chapter 6 discusses the analysis of mode 1-4 arrivals for the $400 \mathrm{~Hz}$ source and the mode 1-3 arrivals for the $224 \mathrm{~Hz}$ source. The narrow pulse width of the $400 \mathrm{~Hz}$ source provided much better data, and it receives a greater share of our attention. Chapter 7 lists the conclusions reached and proposes outlines of future investigation. 


\section{Chapter 2}

\section{Data Collection}

\subsection{Experiment Geometry}

The sixteen element WVLA, moored in 70.5 meters of water off the New Jersey coast, operated continuously for seven days. Tomography sources with carrier frequencies of 224 and $400 \mathrm{~Hz}$ were moored about $32 \mathrm{~km}$ shoreward on a compass heading of $300^{\circ}$ and transmitted to the WVLA. The resulting acoustic path was anti-parallel to the primary propagation direction for shelf generated internal waves. The temperature profile in the vicinity of the WVLA was monitored by six thermistors attached to the array at various depths. Figure 2-1 illustrates the 2-D geometry of the experiment.

\subsection{Source Signals}

The $400 \mathrm{~Hz}$ (100 Hz bandwidth) tomography source produced a 511 digit, phasemodulated signal sequence with a digit length of 4 cycles, for a total of 5.11 seconds per sequence. The sequence was repeated 23 times during each transmission, for a transmission time of 117.53 seconds. The $400 \mathrm{~Hz}$ transmissions started on the hour and were repeated every six minutes. They were received at the WVLA about 22 seconds later. 


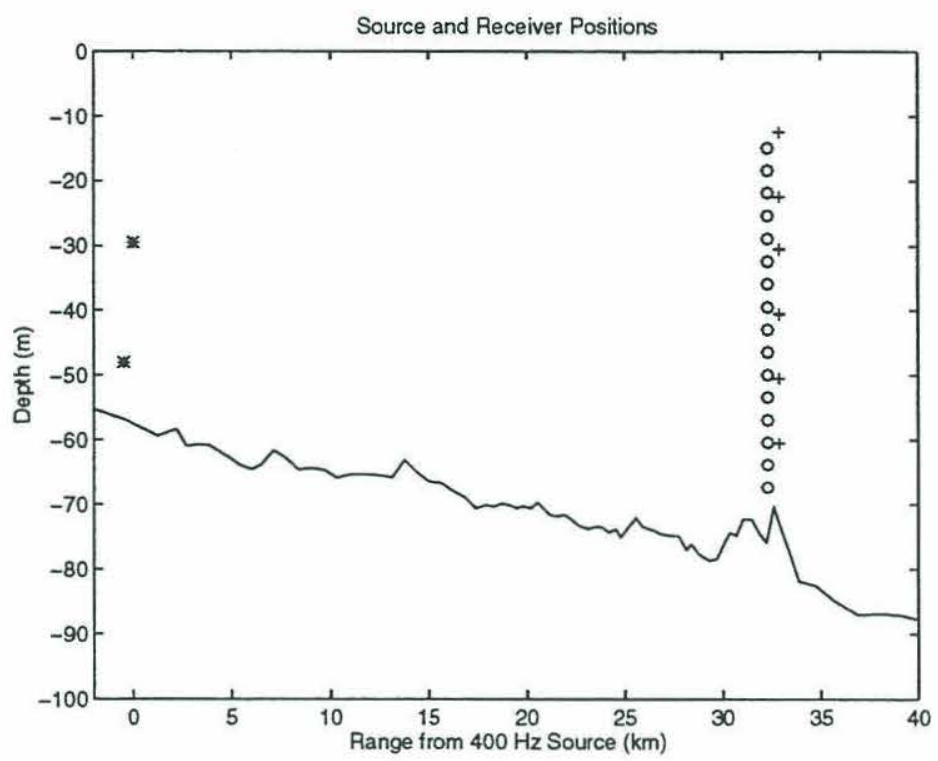

Figure 2-1: Source positions are noted by "*". The $400 \mathrm{~Hz}$ source is the shallower of the two. WVLA hydrophone positions are noted by "o" and the thermistors on the array are marked as "+".

The $224 \mathrm{~Hz}$ (16 Hz bandwidth) tomography source produced a 63 digit sequence, phase-modulated signal with a digit length of 14 cycles, for a total of 3.9375 seconds per sequence. The sequence was repeated 30 times during each transmission, for a transmission time of 118.125 seconds. The transmissions were repeated every five minutes, starting on the hour, five after, etc. They were received at the WVLA about 22.5 seconds later.

\subsection{Receiving Array}

The WVLA had hydrophones spanning water depths from 14.9 to $67.4 \mathrm{~m}$. The hydrophones could not be placed any higher in the water column without experiencing surface wave effects. The sixteen hydrophones were sampled at $1395.089286 \mathrm{~Hz}$ six of the seven days the array was deployed. The sample rate for the remaining period was $1953.1250 \mathrm{~Hz}$. The data were normally telemetered back to a moored research vessel ( $\mathrm{R} / \mathrm{V}$ Oceanus) stationed several kilometers away, but a limited amount of internal 


\begin{tabular}{|l|c|c|c|}
\hline $\begin{array}{l}\text { Tape } \\
\text { Number }\end{array}$ & $\begin{array}{c}\text { Number } \\
\text { of Files }\end{array}$ & $\begin{array}{c}\text { First } \\
\text { File }\end{array}$ & $\begin{array}{c}\text { Last } \\
\text { File }\end{array}$ \\
\hline 1 & 16 & $210-19: 54$ & $211-10: 48$ \\
2 & 7 & $211-16: 43$ & $211-22: 58$ \\
3 & 2 & $212-01: 09$ & $212-01: 11$ \\
4 & 1 & $212-01: 50$ & $212-01: 50$ \\
5 & 14 & $212-03: 27$ & $212-17: 01$ \\
6 & 32 & $213-02: 02$ & $215-06: 01$ \\
7 & 29 & $215-06: 27$ & $216-11: 41$ \\
8 & 18 & $216-13: 04$ & $217-06: 49$ \\
9 & 34 & $210-19: 50$ & $214-05: 13$ \\
10 & 30 & $214-00: 34$ & $217-17: 50$ \\
\hline
\end{tabular}

Table 2.1: Data set outline for the WVLA. Tapes 1 through 8 contain telemetered data that is sometimes broken up when the ship was out of range. Tapes 9 and 10 contain internally recorded data that fills in most of the gaps in the telemetered data.

storage was available and automatically utilized when the ship was out of range. The non-integer sample rates were dictated by the system's analog to digital converter. The equipment provided a very accurate sample rate, but the sample frequencies were limited to

$$
\text { Sample Rate }=\frac{5 \mathrm{MHz}}{256 * n_{1} * 2^{n_{2}}}
$$

In our case, $n_{1}=7$ or 5 , and $n_{2}=1$.

The data were stored on tapes, with about 27 files per tape. Each file contains up to 160 records which are approximately 1 megabyte each. The records consist of 32768 two byte digitized voltage samples for each of the sixteen hydrophones. The sample rate of $1395.089286 \mathrm{~Hz}$, yields about 23 seconds of data per record and 62 minutes of data per file. Seven days of data required over 25 gigabytes of storage, and an outline of the entire data set is given in Table 2-1.

The combination of the $1395 \mathrm{~Hz}$ sample rate and sequence length for the 400 $\mathrm{Hz}$ source yields 7128.9063 samples per sequence, and the $224 \mathrm{~Hz}$ arrivals require 5493.1641 samples per sequence. Both of these numbers are rather cumbersome to 
deal with. An ideal combination of sequence length and sample rate would produce a radix-2 integer number of samples per sequence. During the period from about $1600 \mathrm{Z}$ day 213 to $1600 \mathrm{Z}$ on day 214, the sample rate was shifted from 1395 to $1953 \mathrm{~Hz}$, and the resulting files only cover 44 minutes. Seven transmissions are removed from each $160 \mathrm{Mb}$ file during this period.

There are two further data considerations. First is the location of the header on each file. The telemetered data files have headers at the beginning of the files. The internally recorded data have their headers are at the end. Second, as a consequence of the shifts between telemetered and internally recorded data, some files with less than $160 \mathrm{Mb}$ were created during both the 1395 and $1953 \mathrm{~Hz}$ sampling periods. These files obviously contain fewer transmissions.

\subsection{Signal Isolation}

\subsubsection{Sequence Capture}

The $400 \mathrm{~Hz}$ arrivals at the WVLA can be analyzed one sequence at a time, but of the 23 sequences in a transmission, only 22 full sequences can be captured for analysis. The loss of one sequence is required to compensate for the uncertainty in the actual arrival time of the beginning of the first sequence in a transmission. The transmitted sequences have an ideal circular autocorrelation property that is unaffected by the starting point in a captured sequence.

In our case, sequence capture is accomplished by starting the first capture at a fixed time $(26.85$ secs $)$ after the beginning the transmission. The fixed time for the first sample was chosen through a series of trials to be about 400 samples (depending on the particular transmission) or $280 \mathrm{msec}$ prior to the start of the arrival of the second sequence. The earlier parts of the first sequence are discarded, and the last 400 samples are clipped from the second sequence to provide the data buffer for the beginning of the third sequence. This process continues through the sequences until 
the last 400 samples of the $23 \mathrm{rd}$ sequence are clipped and discarded. The 22 sequences obtained in this manner are rounded to 7129 samples long, and have basically been circularly shifted to the right (i.e. delayed) by about 400 samples with respect to the transmitted sequence. In a similar manner, 29 sequences of the 30 transmitted can be captured from each $224 \mathrm{~Hz}$ transmission. The $224 \mathrm{~Hz}$ sequences are rounded to 5493 samples for analysis.

Changes in travel-time are estimated by capturing sequences using a fixed time delay after transmission and detecting the resulting circular shifts in the captured sequences. There are a non-integer number of samples between transmissions, so choosing the first sample to capture in a sequence creates an error between the desired fractional sample number and the allowable integer sample numbers. A correction for the time and phase error introduced by the sample error can be made by multiplying

the base-band signal in the frequency domain by $e^{i 2 \pi\left(f+f_{c}\right) \Delta t}$, where $f$ is the base-band frequency, $f_{c}$ is the carrier frequency, and $\Delta t$ is the sample error divided by the sample rate. This correction step is accomplished in conjunction with pulse-compression.

\subsubsection{Pulse Compression}

The captured $400 \mathrm{~Hz}$ sequences are demodulated to baseband and then low-pass filtered by windowing in the frequency domain with a $90 \mathrm{~Hz}$ pass-band Blackman window. Pulse compression is also accomplished in the frequency domain through multiplication with the conjugate spectrum of the transmitted sequence. This is equivalent to a matched-filter circular convolution in the time domain [11].

If sequence lengths of 5493 and 7129 are maintained, mixed-radix DFTs must be used in the transforms between the time and frequency domains. These mixed-radix DFTs are too slow for practical application, so the data was zero-padded to a length of 8192 in order to employ a radix-2 FFT.

The circular convolutions of delayed and zero-padded signals with zero-padded transmitted sequences are not ideal. The resulting sidelobes (Figure 2-2) stem from 

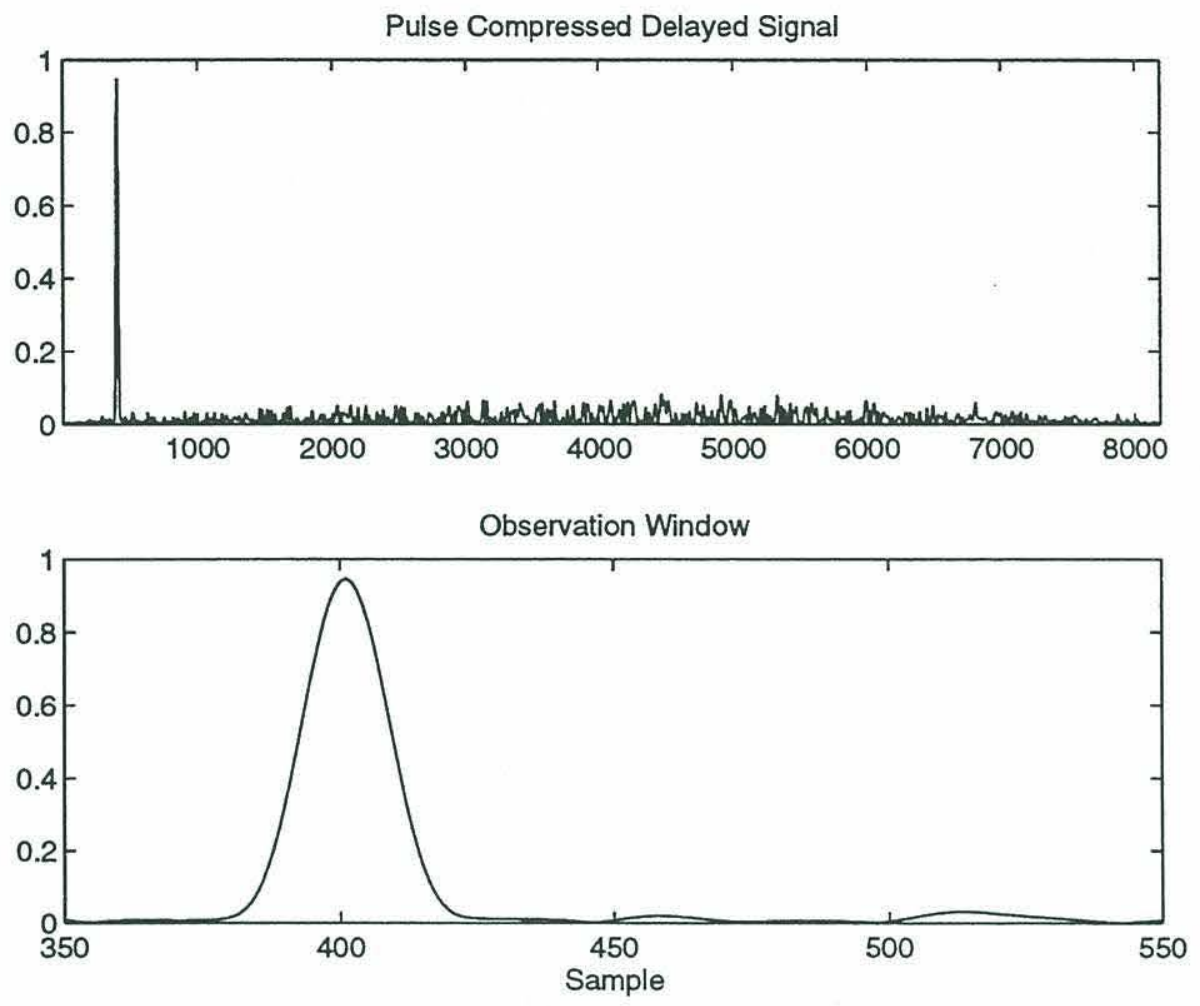

Figure 2-2: Circular convolution of an ideal delayed $400 \mathrm{~Hz}$ transmitted sequence with an ideal non-delayed transmitted sequence. Both sequences have been zero-padded from length 7129 to length 8192 . The lower frame is a window of the full output above.

offsets in the location of the zeros. The sidelobe effects were eliminated from all analyses presented through SNR discrimination.

It is not necessary to save the entire output of the pulse compressed data. The relevant portions of the data are limited to an area around the peak arrival that encompasses the spread of energy caused by mode dispersion and coupling. A 200 sample ( $143 \mathrm{msec}$ ) observation window like that shown in Figure 2-2 is sufficient to capture the spread expected from modal dispersion over $32 \mathrm{~km}$, but an additional $70 \mathrm{msec}$ is required to accommodate the wander. Figure 3-3 shows some typical saved outputs of $400 \mathrm{~Hz}$ pulse-compressed arrival patterns for the hydrophones at the WVLA. Due to its wider pulse width, $358 \mathrm{msec}$ are saved for the $224 \mathrm{~Hz}$ arrivals. 

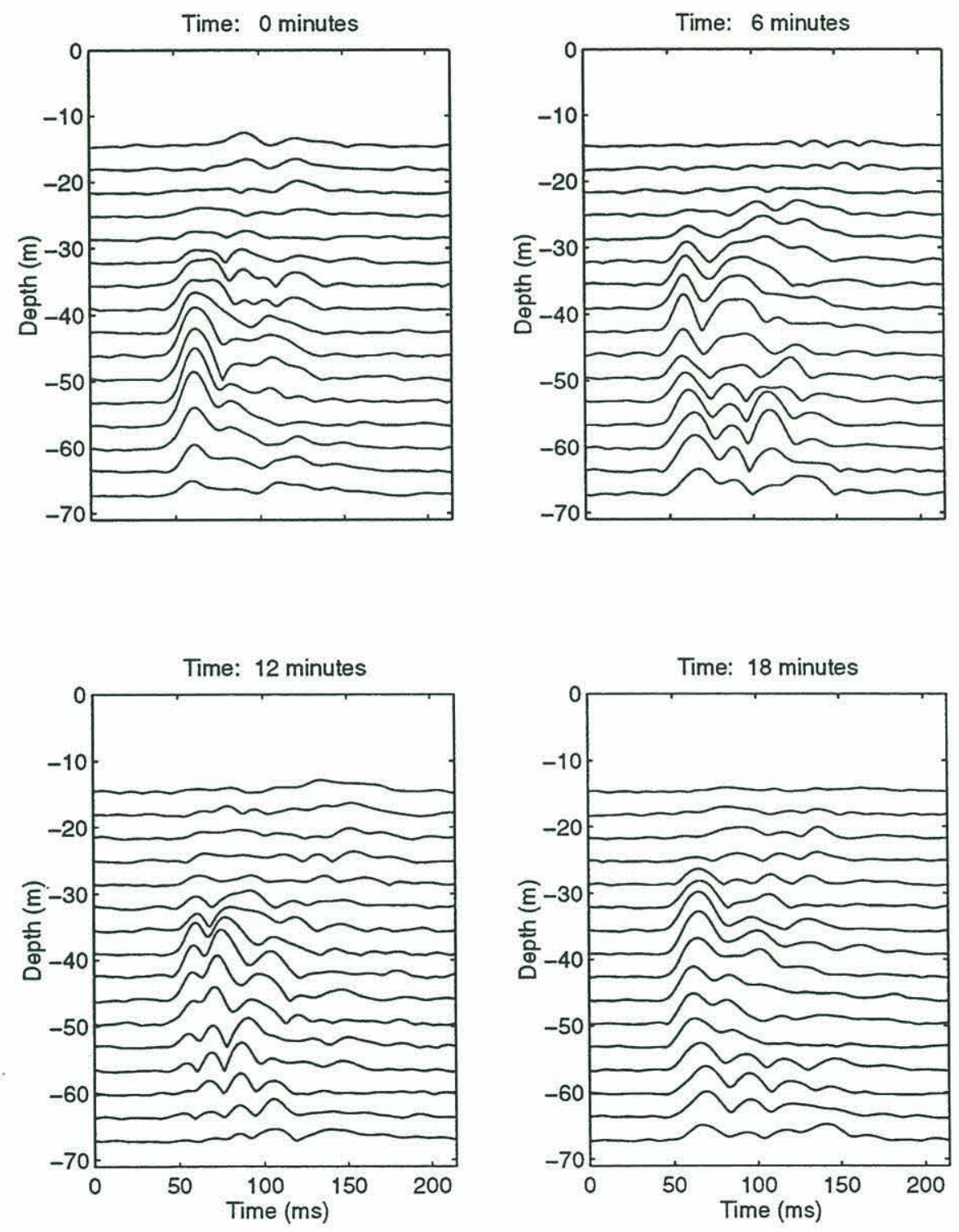

Figure 2-3: Typical $400 \mathrm{~Hz}$ arrivals at the WVLA hydrophones. The sets of 16 arrivals are separated by 6 minutes. 


\section{Chapter 3}

\section{Mode Filtering}

\subsection{Normal Modes}

The two most frequently used representations of sound propagation in ocean acoustic waveguides are rays and normal-modes. Ray theory is perhaps more intuitive, but it is a high frequency approximation that is better suited to situations where the acoustic wavelength is small compared to the dimensions of the sound channel. Our low frequencies and shallow waveguide are better suited to a modal analysis than ray theory.

The modal analysis of $400 \mathrm{~Hz}$ acoustic arrivals at the WVLA begins by treating the acoustic signal at each hydrophone as a sum of vertical modes

$$
p_{h}(t)=\sum_{n=1}^{N} A_{n}(t) \phi_{n}\left(z_{h}\right)
$$

where $\phi_{n}(z)$ is the mode shape, $A_{n}(t)$ is the mode coefficient, and $z_{h}$ is the depth of the hydrophone in question. The number of propagating normal modes, $N$, as well as the mode shapes themselves are frequency dependent. The mode shapes are analogous to the eigenmodes of a vibrating string, and are formed by a constructive vertical interference pattern caused by a pair of up and down going plane-waves with the 
same horizontal wave number. The highest possible normal mode number is roughly equivalent to the sound channel depth divided by the acoustic wavelength, where the up and down going pair of plane-waves would be propagating with a horizontal wave number approaching zero.

The six thermistors on the WVLA, which are sampled every 30 seconds, provided the soundspeed profiles needed for computing a time-dependent set of mode shapes using the KRAKEN [12] normal-mode code. The basis for this code, a numerical normal-mode solution of the Helmholtz equation at frequency $\omega$,

$$
\left[\nabla^{2}+k^{2}(x, y, z, \omega)\right] p(x, y, z, \omega)=0
$$

where $p(r, \omega)$ is the Fourier component of the pressure field, can be found in Jensen et al. [13].

The KRAKEN [12] mode shapes are orthonormal in the sense that,

$$
\int \frac{\phi_{m}(z) \phi_{n}(z)}{\rho(z)} d z=\delta_{m n}
$$

so given $p(z, t), A_{n}(t)$ can be readily computed by applying the operator

$$
\int(\cdot) \frac{\phi_{n}(z)}{\rho(z)} d z
$$

Since the pressure field, $p(z, t)$, is only sampled at the sixteen hydrophone locations, the integral computation of the mode coefficients, $A_{n}(t)$, must be approximated by

$$
A_{n}(t) \simeq \sum_{h=1}^{16} \frac{p\left(z_{h}, t\right) \phi_{n}\left(z_{h}\right) \Delta z}{\rho\left(z_{h}\right)}
$$

where $\Delta z$ is the spacing between hydrophones. This discrete sum can be easily implemented through matrix multiplication of mode shape vectors with 16 channel hydrophone data vectors. This process of converting the 16 channels of hydrophone data into $\mathrm{N}$ normal mode arrivals is referred to as narrowband direct-projection (NBDP) 
mode-filtering.

An alternative, broadband direct-projection (BBDP) mode-filtering operates in the frequency domain, approximating the Fourier components of the mode arrivals as

$$
A_{n}\left(f_{j}\right) \simeq \sum_{h=1}^{16} \frac{p\left(z_{h}, f_{j}\right) \phi_{n}\left(z_{h}, f_{j}\right) \Delta z}{\rho\left(z_{h}\right)}
$$

where

$$
p\left(z_{h}, f_{j}\right)=\sum_{m} p\left(z_{h}, t_{m}\right) e^{-i 2 \pi\left(f_{j}\right) t_{m}} \Delta t .
$$

The time domain mode coefficients can then be recovered through

$$
A_{n}\left(t_{m}\right)=\sum_{j} A_{n}\left(f_{j}\right) e^{i 2 \pi\left(f_{j}\right) t_{m}} \Delta f
$$

The benefits of this method over NBDP depend upon the frequency dependence of the mode shapes over the bandwidth of the arriving signal. The cost of BBDP is in computation time; over 4000 different sets of narrowband mode filters are required for each frequency to cover the time-dependence seen in six days of data, so in the ideal extreme of a different set of $\phi_{n}\left(f_{j}\right)$ s for each of the 1000 frequency bins that cover the bandwidth of the signal, 4 million sets of mode filters would be have to be computed! The extreme case is not feasible, but there are compromises that can be explored.

Either DP method should work very well for the low modes that are adequately sampled by the WVLA. A typical SWARM mode 1 shape will yield,

$$
\sum_{h=1}^{16} \frac{\phi_{1}\left(z_{h}\right) \phi_{1}\left(z_{h}\right) \Delta z}{\rho\left(z_{h}\right)}=0.9989
$$

very close to the ideal output of 1.0. Higher modes have more energy outside the depths sampled by the WVLA, so the above "auto-filtration" degrades, yielding outputs of $0.9949,0.9881,0.9803,0.9681,0.9269$, and 0.8243 for modes 2 through 7 , respectively. The higher modes suffer more from partial coverage than they do from 
undersampling, so a simple output gain factor (like dividing the filter output by its auto-filtration level) may be useful if level comparisons between mode arrivals are to be made. However, an output gain factor will not provide relief from modal-crosstalk, which is also inherent with undersampling and partial coverage.

One must keep in mind that mode coefficient outputs of the mode-filtration are dependent on the quality of both the computed mode shapes and the measured pressure field. The most significant error associated with pressure field measurements is uncompensated array tilt. Deviations from a perfectly vertical array will cause phase differences between hydrophones that degrade the mode coefficient computations.

\subsection{Array Tilt}

The designed navigation system for the WVLA was extensive and complete, with three transponder travel-times recorded at four hydrophones on the array. Unfortunately, the travel-times from one of the transponders to three of the hydrophones did not record properly, leaving just one channel (the shallowest hydrophone) fully navigated. Moreover, all of the navigation travel-times appear to be heavily influenced by small-scale temperature fluctuations caused by internal waves. The waves caused navigation travel-time changes that were three to five times larger than those expected from mooring motion. The time scale of these travel-time changes is on the order of the navigation sample rate (once every four minutes), consistent with soliton passage. The longer time-scale navigation travel-time changes are similarly out of proportion with expected motions of the array. They are probably due to path dependent temperature fluctuations, as well; thus these navigation data are considered largely unusable.

We can try to estimate the array tilt by hydrodynamics. The length of the of the mooring is approximately 60 meters and it has net buoyancy of 3230 Newtons. Assuming laminar flow in calculating the form drag for the cable and flotation sphere, 
a barotropic current will cause upper hydrophone displacements that are roughly proportional to the square of the current speed, giving 1 meter at $30 \mathrm{~cm} / \mathrm{s} ; 2$ meters at $40 \mathrm{~cm} / \mathrm{s}$; and 3 meters at $50 \mathrm{~cm} / \mathrm{s}$. The current records obtained from a bottommounted acoustic Doppler current meter (ADCP), moored about $3 \mathrm{~km}$ from the WVLA, showed barotropic currents that seldom exceeded $30 \mathrm{~cm} / \mathrm{s}$, with occasional baroclinic currents from large solitons of up to $50 \mathrm{~cm} / \mathrm{s}$.

The effects of baroclinic currents on mooring motion are hard to predict, but the movement at the top hydrophone is certainly less than what would be produced by a barotropic current of the same magnitude. This line of reasoning predicts mooring motions that are generally less than a meter in amplitude at the top hydrophone (equivalent to 1 degree of tilt) with occasional excursions that might approach 2 meters. A more detailed analysis of the WVLA navigation data is provided in Appendix A.

\subsection{Mode Filtering}

\subsubsection{Errors from Array Tilt}

As mentioned earlier, phase differences caused by array tilt will cause errors when the 16 channels of acoustic data from the WVLA are converted to normal mode arrivals through mode-filtering. Figure 3-1 illustrates the effects of array tilt on some typical $400 \mathrm{~Hz}$ mode-filter outputs; the inputs are pure mode shapes from the same sound speed profile (SSP) as the mode-filter. With no tilt, all four mode filters do an exceptional job of passing their respective mode and rejecting all others. The mode-filters continue to perform acceptably for small deflections (less than 1 meter) of the upper hydrophone, but most become significantly degraded for deflections approaching 2 meters. With the exception of mode 2 rejection, the mode 1 filter performs robustly all the way to 2 meters of tilt. 


\section{Typical Tilt Dependence}
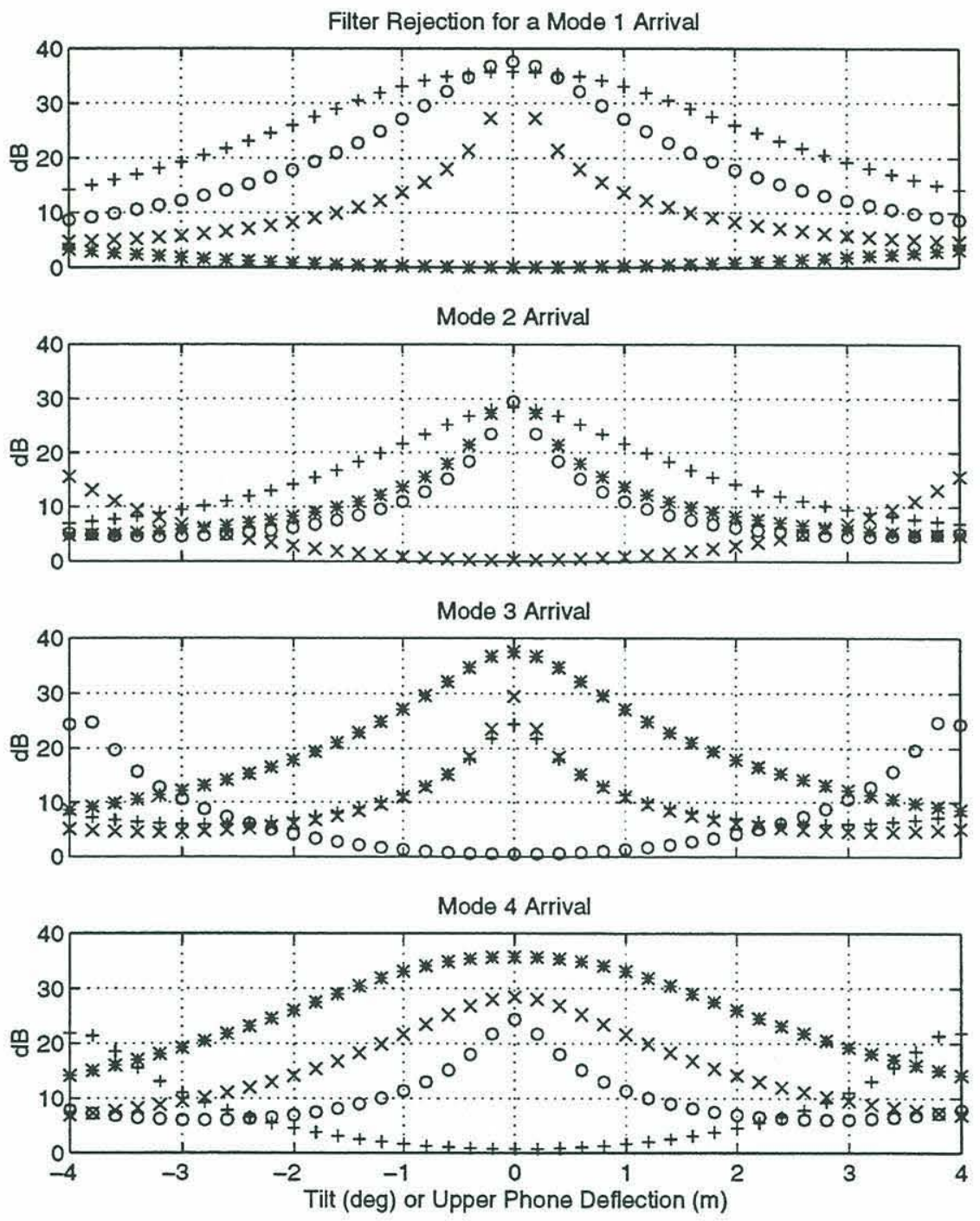

Figure 3-1: Mode rejection levels from mode-filter outputs, $A_{1}(*), A_{2}(\mathrm{x}), A_{3}(\mathrm{o})$, and $A_{4}(+)$, as a function of array tilt. The four plots represent tilted inputs of pure mode 1 , mode 2 , mode 3 , and mode 4 arrivals. These plots use mode filters and mode arrivals generated from the same typical SWARM SSP. 


\section{Additional Error from Mode Shape Differences}

Another source of error for the mode filter is the difference between the sound speed profile used in creating the mode filter and the actual sound speed profile during the acoustic arrival. The 22 sequences of a transmission are split into two groups of eight and one group of six sequences for further analysis. The mode filter for each group is calculated by using the WVLA thermistor record samples whose sample time is closest to the arrival time of the middle sequence of the group. The thermistors on the WVLA are sampled every 30 seconds, and an eight sequence group covers about 41 seconds. In only three of the 22 sequences is the arrival of a particular sequence offset from the sample time of the mode-filter by more than 30 seconds, and the worst of these three is 42 seconds. Under most circumstances this error will be insignificant, but during the passage of a soliton, offsets of 30 seconds can produce noticeable effects.

\subsubsection{Time Dependence of Filter Performance}

Even with zero-tilt and no difference in mode shapes, modal cross-talk cannot be avoided completely (see Figure 3-1 at zero-tilt). This minimum level of cross-talk is borne of under sampling and partial coverage (i.e. discretization error). The exact amount of cross-talk is dependent on the mode shapes in question, and, as such, the zero-tilt filter performance at the WVLA is time varying. Figure 3-2 plots the "Best Case" (i.e. zero-tilt with no time offset) performance of the mode 1-4 filters calculated from thermistors on the WVLA during the period between about 1300 on yearday 216 and 0130 on yearday 217. The temporal variation is significant, but the cross-mode rejection levels never drop below $20 \mathrm{~dB}$. The mean "Best Case" mode filter rejection 


\section{Best Case Time History}
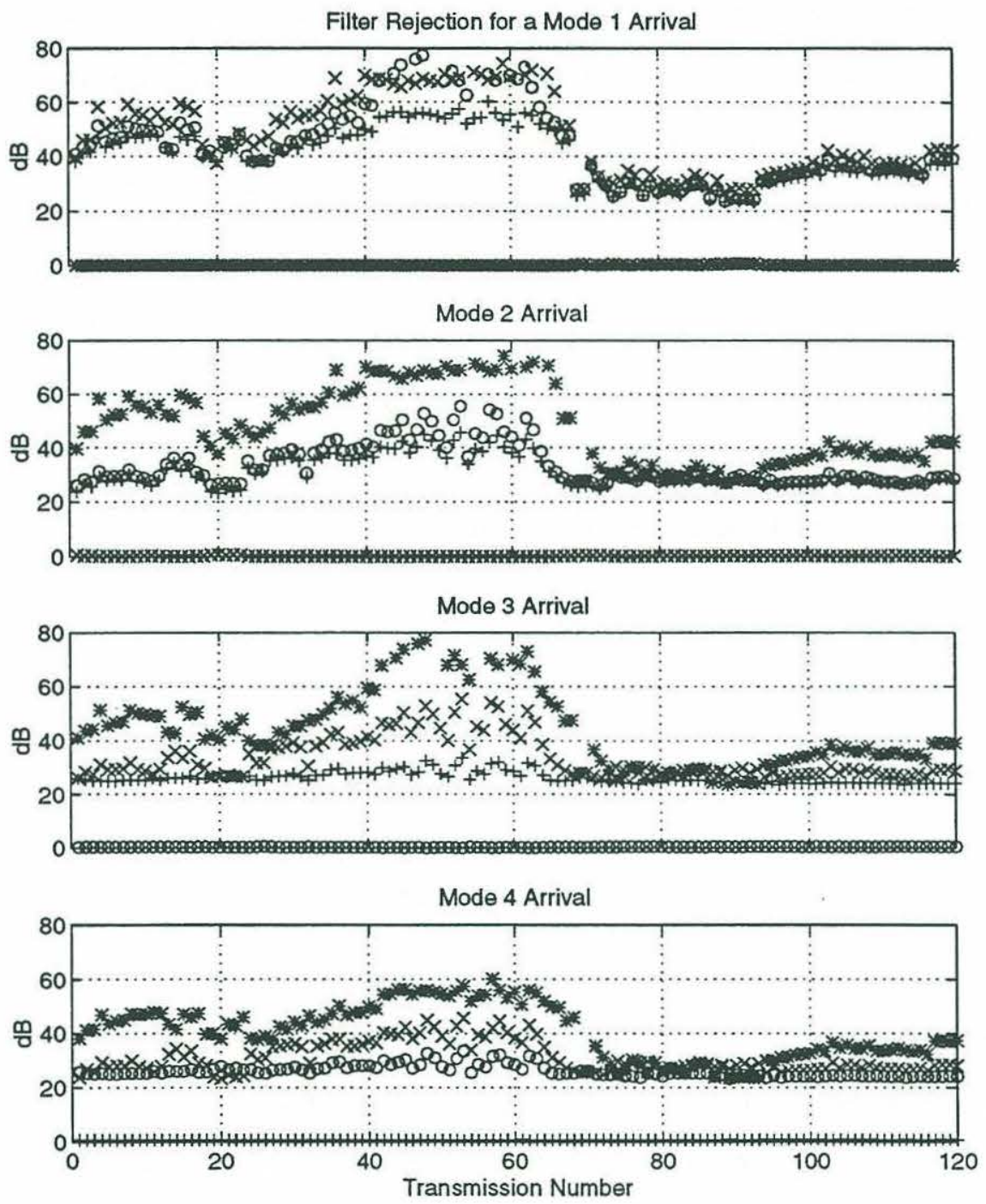

Figure 3-2: Mode rejection levels from mode-filter outputs, $A_{1}(*), A_{2}(\mathrm{x}), A_{3}(\mathrm{o})$, and $A_{4}(+)$, as a function of transmission number. The four plots are based on a series of untilted inputs of pure mode 1 , mode 2 , mode 3 , and mode 4 arrivals, where the mode filters and mode arrivals are generated from the same series of SSPs. 
levels from Figure 3-2 are

$$
\left\langle-20 \log \left(\sum_{h=1}^{16} \frac{\phi_{i}\left(z_{h}, t\right) \phi_{j}\left(z_{h}, t\right) \Delta z}{\rho\left(z_{h}\right)}\right)\right\rangle=\left[\begin{array}{rrrr}
0 & 47 & 46 & 40 \\
49 & 0 & 34 & 31 \\
46 & 34 & 0 & 26 \\
40 & 31 & 26 & 1
\end{array}\right]
$$

in matrix form, where levels are in $\mathrm{dB}$.

The above performance levels, as stated, are optimistic; they may be taken as upper bounds. The "Worst Case" performance levels for this same period, shown in Figure 3-3, can be thought of as lower bounds. They assume a constant tilt of one degree and a 30 second offset between the arrivals and the mode filters. As stated before, tilts may exceed $1 \mathrm{~m}$ from time to time and time offsets of up to 42 seconds are possible, but the "true worst case" is a fairly elusive concept; mode shapes are only tabulated in 30 second intervals, and the tilt distribution tail function is difficult to quantify. Given all this, the term "nearly worst case" might be better; still, the tilt and time offset parameters chosen for Figure 3-3 will bound at least 90 percent of the arrivals, and parameters sufficient to bound the performance 100 percent of the time would provide little in terms of a useful comparison.

The "Worst Case" filter performance is significantly reduced, relative to the "Best Case" scenario. Most of this reduction can be attributed to the assumed one degree of tilt, but, occasionally, the passage of a soliton at the WVLA causes a large rapid change in mode shapes during a mode arrival. The assumed 30 second time difference between arrivals and filters can then cause an additional marked drop in performance. The drop seen at transmission number 27 is one such occurrence. Overall, the "Worst Case" mode filter rejection levels from Figure 3-3 are still quite acceptable, but there are indications that the quality of individual arrivals may be relatively poor from 


\section{Worst Case Time History}

Filter Rejection for a Mode 1 Arrival
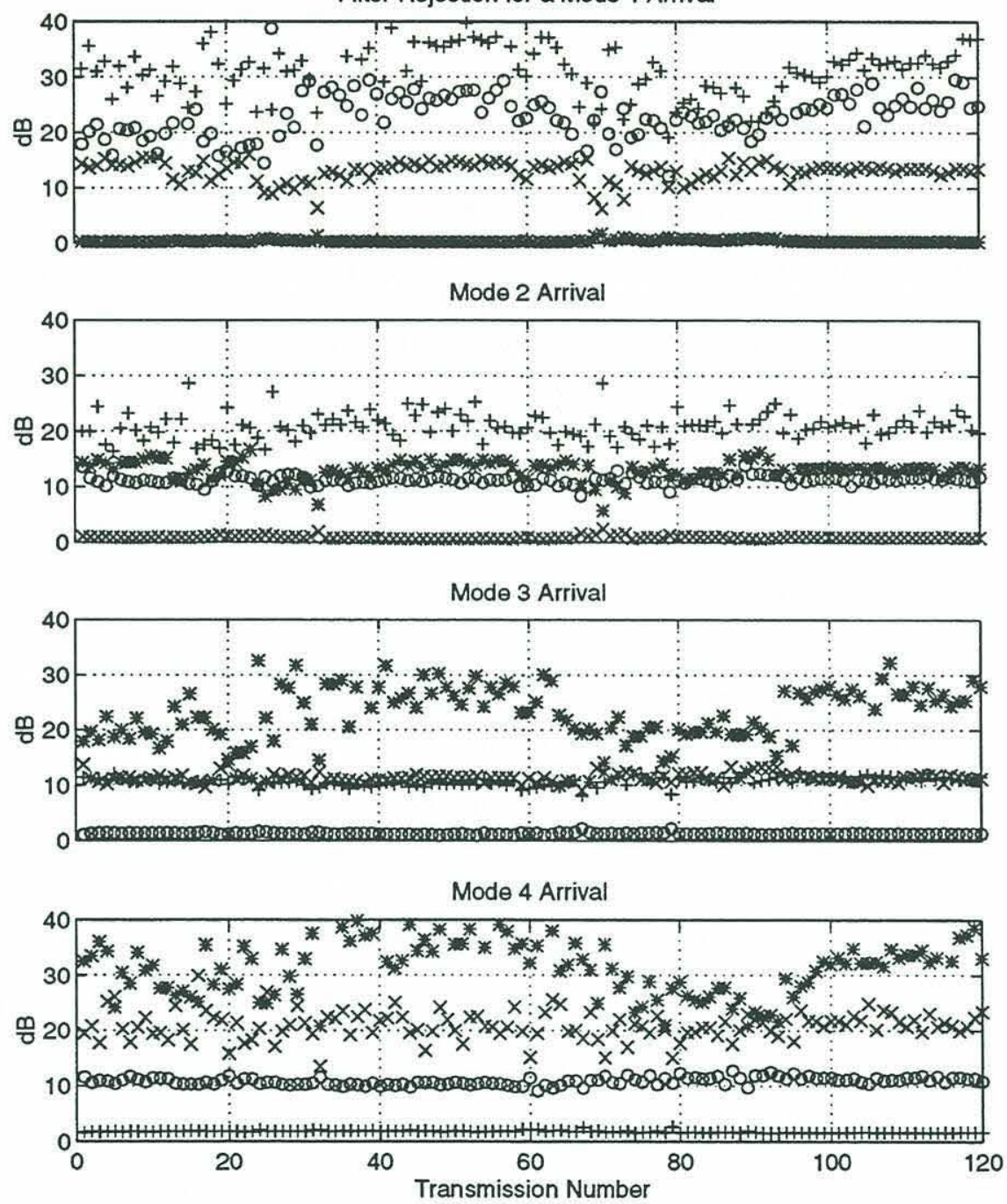

Figure 3-3: Mode rejection levels from mode-filter outputs, $A_{1}(*), A_{2}(\mathrm{x}), A_{3}(\mathrm{o})$, and $A_{4}(+)$, as a function of transmission number. The four plots are based on 1 degree tilted inputs of pure mode 1 , mode 2 , mode 3 , and mode 4 arrivals, using mode filters and mode arrivals generated from the SSPs separated by 30 seconds. 
time to time. The mean "Worst Case" values in matrix form are (again in dB levels)

$$
\left\langle-20 \log \left(\sum_{h=1}^{16} \frac{\phi_{i}\left(z_{h}, t\right) \phi_{j}^{*}\left(z_{h}, t-30\right) \Delta z}{\rho\left(z_{h}\right)}\right)\right\rangle=\left[\begin{array}{rrrr}
0 & 13 & 24 & 32 \\
13 & 1 & 11 & 20 \\
24 & 11 & 1 & 11 \\
32 & 21 & 11 & 2
\end{array}\right] .
$$

Where $\phi_{j}^{*}\left(z_{h}, t-30\right)$ is a one degree tilted version of $\phi_{j}\left(z_{h}, t\right)$ that is also offset in time by 30 seconds.

A reasonable estimate of the expected performance for the four mode filters might be found by taking the average of the mean of both cases. The result is

$$
\left[\begin{array}{rrrr}
0 & 31 & 35 & 36 \\
31 & 1 & 22 & 26 \\
34 & 22 & 1 & 18 \\
36 & 26 & 18 & 1
\end{array}\right]
$$

in matrix form. These levels are quite good, but they must be considered in light of the potential for large differences between modal intensities at the receiver (e.g. the average filtered peak height differential between mode 1 and mode 4 is about $10 \mathrm{~dB}$.)

\subsubsection{Mode Filter Outputs}

As discussed previously, we do not expect to achieve perfect resolution of a given mode arrival, but we may reasonably expect the lower mode-filter outputs to generally reflect the true mode arrival structures. Figure 3-4 shows mode 1-4 filtered outputs for ten sequences, each separated by 6 minutes, over a 54 minute period. Most of the arrival structures do not seem to follow any clearly recognizable patterns, and the temporal variability over a six minute period is quite dramatic. The patterns are basically uncorrelated. 

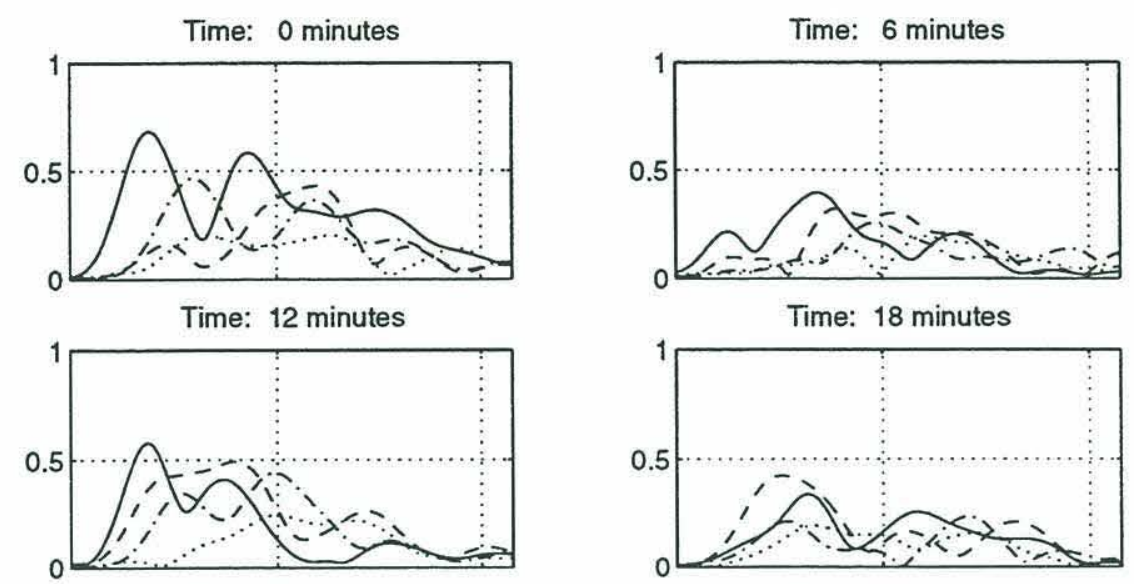

Time: 24 minutes
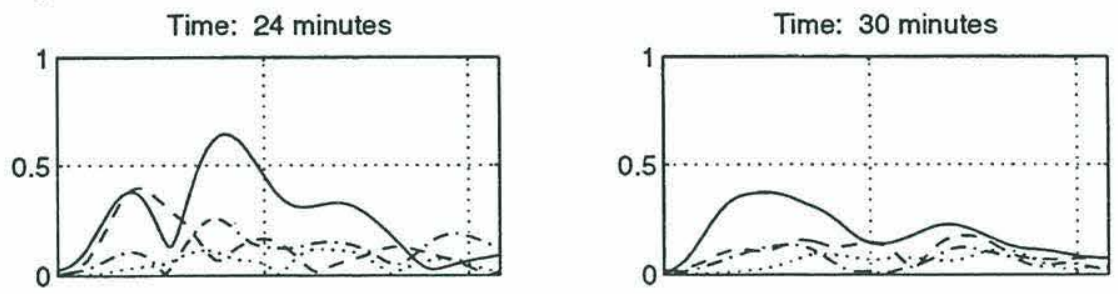

Time: 36 minutes

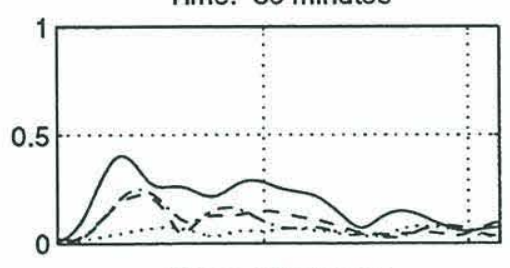

Time: 42 minutes
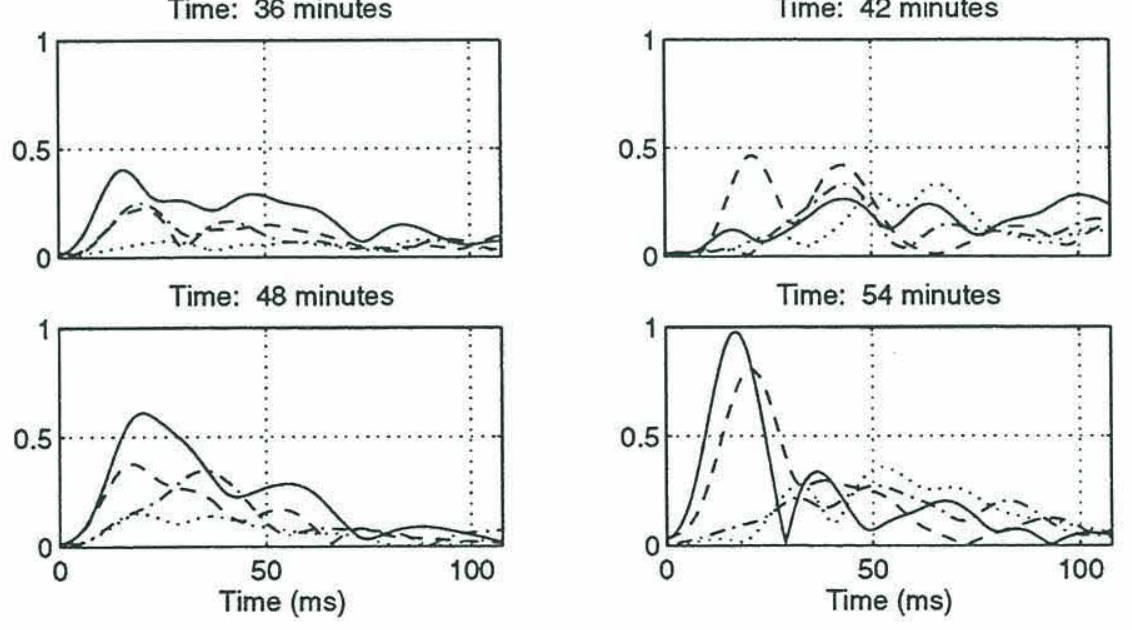

Figure 3-4: Mode Coefficient outputs, $A_{1}$ (solid), $A_{2}$ (dashed), $A_{3}$ (dot-dash), and $A_{4}$ (dotted), as a function of time. The ten plots depict the arrival structure for the first of 22 captured sequences out of ten sequential transmissions. 
Figure 3-5 illustrates the temporal variability of mode arrivals over a shorter time scale by following the evolution of the time zero transmission in Figure 3-4 over a period of 92 seconds. This figure shows some fairly strong correlations between sequences separated by upwards of one minute; a more quantitative measure of the temporal coherence can be reached through computing the correlation coefficient between the first sequence of a transmission and the twenty subsequent sequences in that transmission. Figure 3-6 displays the results of these computations when averaged over ten transmissions. For the analyzed time period, the average $3 \mathrm{~dB}$ down points for the correlation functions fall between 70 and 90 seconds. A good question to try to answer is why the lowest mode decorrelates least quickly and the highest mode decorrelates most quickly. It is probably related to the percentage of scattered energy in each arrival pattern, and it should be investigated further in the future. However, it will not be simple, as the broken sampling schedule at the WVLA is not very conducive to correlation analysis. Significant levels of decorrelation often do not appear over the course of a transmission.

The arrival structures we have observed are clearly indicative of a large amount of mode scattering between the source and the receiver. The source of most of the scattering is very probably the numerous internal wave solitons observed by the thermistors on the WVLA and other moorings deployed during SWARM. A numerical model for mode dispersion and coupling will be a useful tool in identifying the relevant statistics of these complicated arrival patterns. 

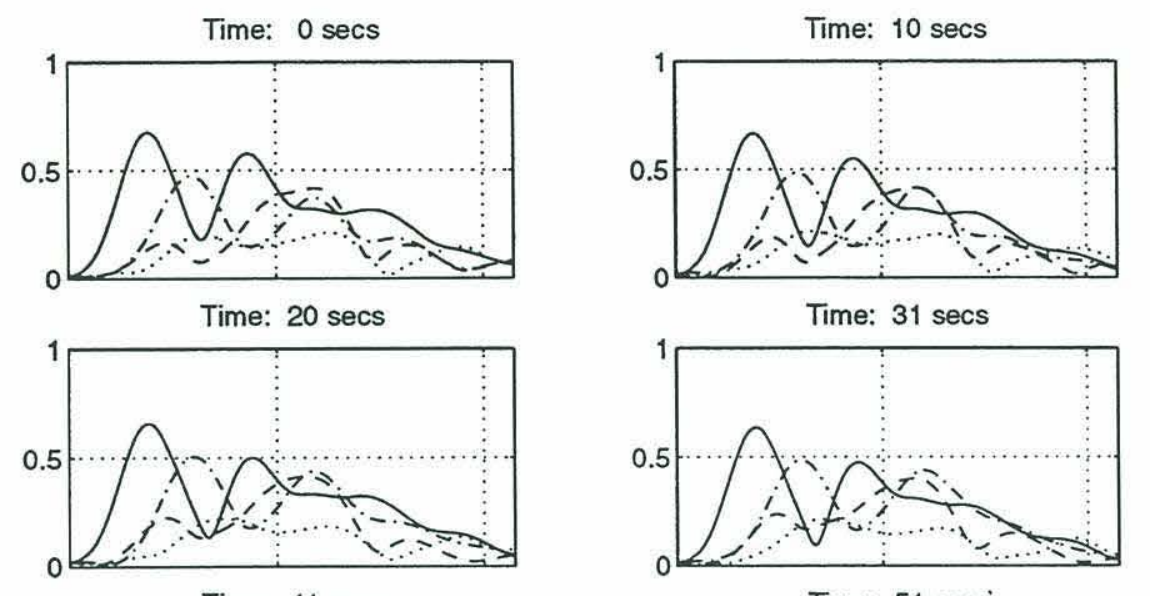

Time: 41 secs

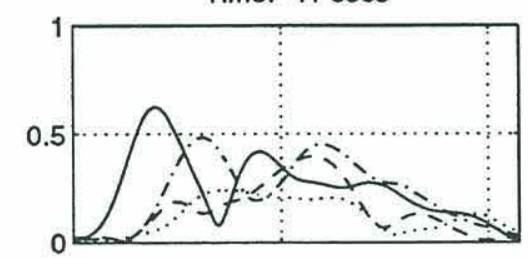

Time: 51 secs
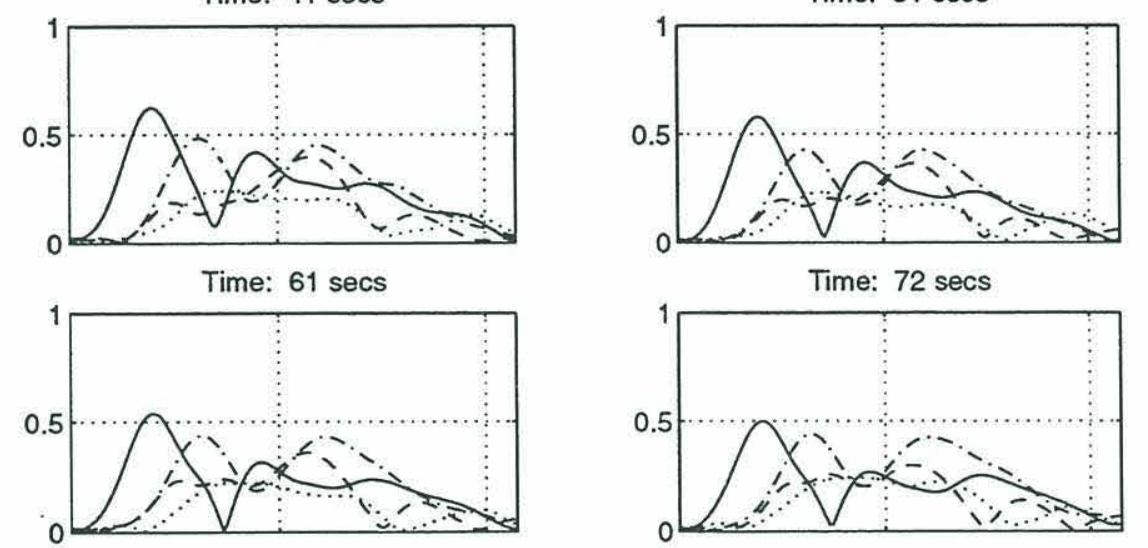

Time: 82 secs

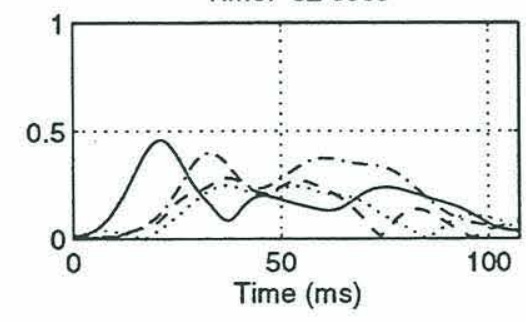

Time: 92 secs

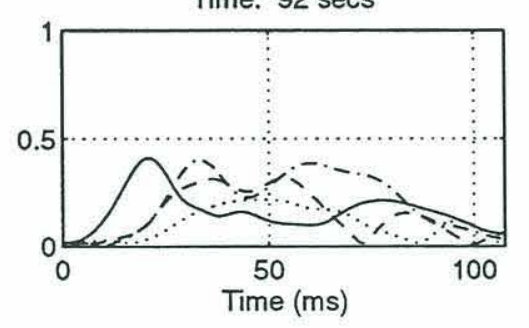

Figure 3-5: Mode coefficient outputs, $A_{1}$ (solid), $A_{2}$ (dashed), $A_{3}$ (dot-dash), and $A_{4}$ (dotted), as a function of time. The ten plots depict the arrival structure for every other sequence from the first to the 19th of 22 captured sequences from the time zero transmission in Figure 3-4. 


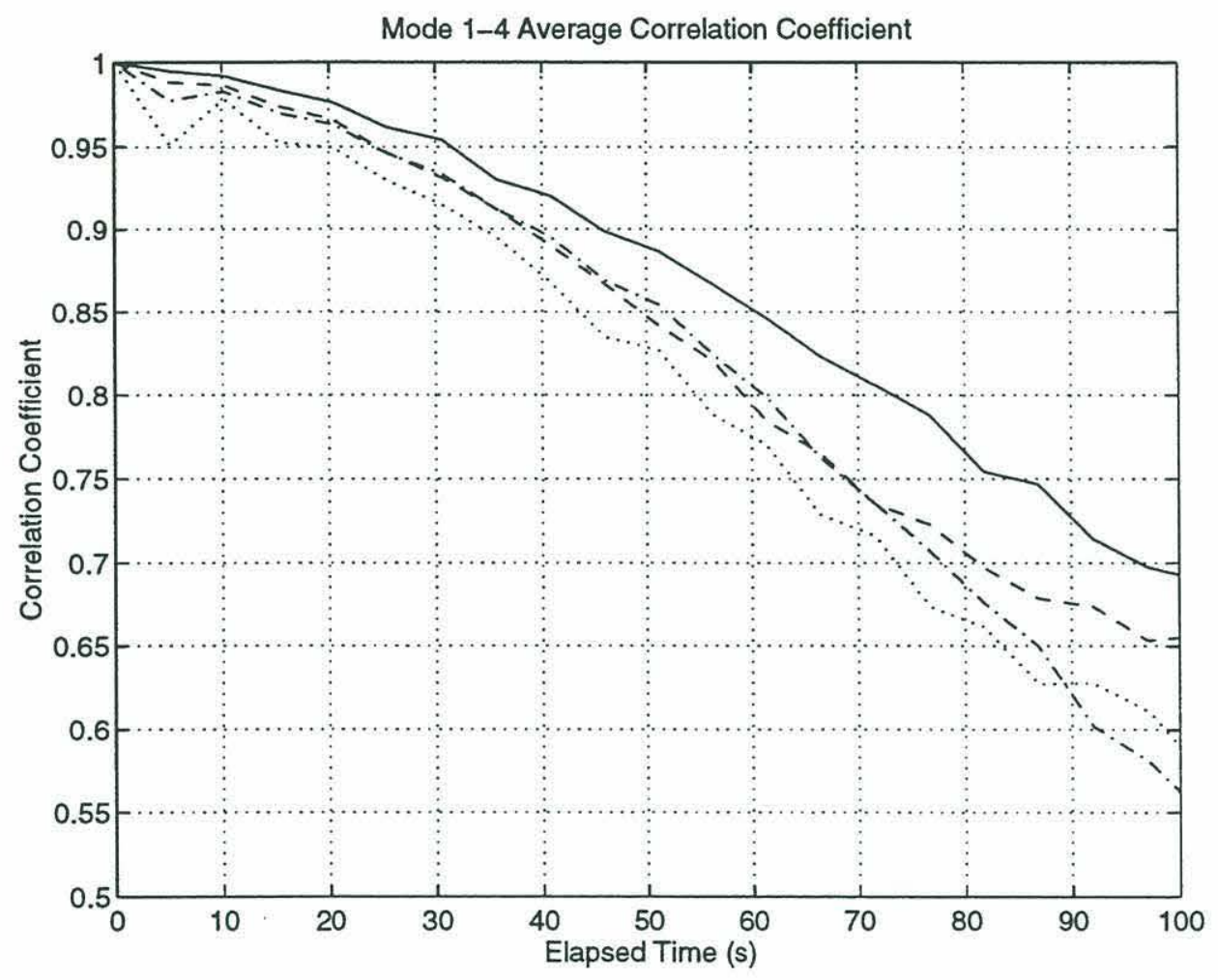

Figure 3-6: Average correlation coefficients for outputs, $A_{1}$ (solid), $A_{2}$ (dashed), $A_{3}$ (dot-dash), and $A_{4}$ (dotted), as a function of elapsed time. The average is taken over the ten transmissions of Figure 3-4. 


\section{Chapter 4}

\section{A Simple Scattering Model}

Several avenues are available for developing a model that exhibits the main features of the scattering behavior seen in the acoustic data. We will start with the simplest possible time domain model and examine some of its behavior; we will then transition to a broadband model that includes added complexity.

\subsection{Normal Mode Pulse Propagation}

In order to perform analysis of the acoustic arrivals at the WVLA, the acoustic signal transmitted from the $400 \mathrm{~Hz}$ source is treated as a sum of vertical modes

$$
p_{s}(z, t)=A_{n} \phi_{n}(z) \tilde{p}(t)
$$

where repeated indices indicate summation, $\phi_{n}(z)$ is the mode shape, $A_{n}$ is the mode coefficient, and $\tilde{p}(t)$ is the pulse-shape of the transmitted sequence after baseband replica correlation (see Figure 2-2). Each of the modes transmitted can couple into other modes when large horizontal sound speed gradients are encountered between the source and receiver. The large gradients could be caused by temperature fronts, salinity intrusions, or the internal wave solitons which are of particular interest here.

To estimate the coupling of modes across the soliton gradients, an approximate 
single-scatter one-way coupled mode approach from Jensen et al. [13] is employed that uses an arithmetic mean of pressure and velocity matching conditions across the interface to produce the elements of the coupling matrix

$$
C_{l m}(z, t)=\frac{1}{2} \int \frac{\phi_{l}^{j+1}(z) \phi_{m}^{j}(z)}{\rho^{j+1}(z)} d z+\frac{k_{r m}^{j}}{2 k_{r l}^{j+1}} \int \frac{\phi_{l}^{j+1}(z) \phi_{m}^{j}(z)}{\rho^{j}(z)} d z,
$$

where $k_{r m}^{j}$ is the horizontal wave number of mode $m$ in region $j$, and the coupling among mode coefficients is given by

$$
A_{l}^{j+1}=C_{l m}^{j+1} A_{m}^{j},
$$

or in matrix notation,

$$
A^{j+1}=C^{j+1} A^{j} .
$$

Parabolic Equation (PE) simulations by Presig and Duda [2] indicate that the mode coupling caused by a single soliton (whose width is less than about $400 \mathrm{~m}$ ) can be reasonably modeled by a sharp transition into and out of the soliton's sound speed profile (SSP). This sharp interface approximation (SIA), illustrated in Figure 4-1, provides an avenue for treating acoustic transmissions as an ever-increasing sum of mode pulses propagating through the waveguide. Figure 4-1 also shows the idealized SSPs for the regions inside and outside the inverted top hat. The nearly isovelocity background SSP is unrealistic, but its sinusoidal, frequency-independent, mode shapes are useful in this initial, simplistic, look at the problem.

\subsubsection{Total Field Summation}

If there are $J$ trapped modes in both the background and "soliton filled" wave guides, each mode pulse will couple into $J$ further pulses as it transitions from one "environment" to the other, creating an $J^{2}$ increase in the number of pulses for each of the double transitions seen in individual soliton interactions. Extrapolating this line of 

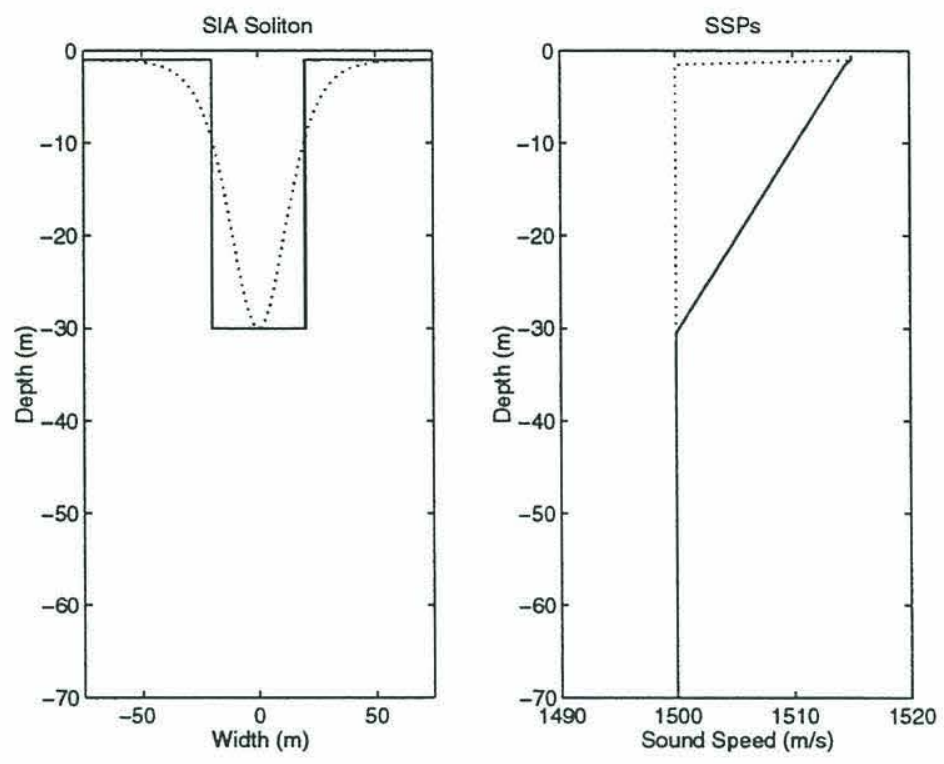

Figure 4-1: An illustration of the SIA approximation for solitons (left), and the idealized SSPs (right) used to model the interior and exterior regions of the SIA soliton. The dotted curve represents the exterior or background region, and the solid curve represents the interior.

thought to a number of soliton interactions yields the following time-domain pulse synthesis representation for the signal at the WVLA

$$
\begin{aligned}
& p_{w v l a}(z, t)=\phi_{a}(z) C_{a b}^{(N)} C_{b c}^{(N-1)} C_{c d}^{(N-2)} \ldots C_{j k}^{(1)} A_{k} \\
& \times \exp \left[i k_{r a}^{N}\left(r^{w v l a}-r^{N}\right)+i k_{r b}^{N-1}\left(r^{N}-r^{N-1}\right)+\cdots+i k_{r k}^{0}\left(r^{1}-r^{0}\right)\right] \\
& \times \tilde{p}\left(t-\frac{\left(r^{w v l a}-r^{N}\right)}{u_{a}^{N}}-\frac{\left(r^{N}-r^{N-1}\right)}{u_{b}^{N-1}}-\cdots-\frac{\left(r^{1}-r^{0}\right)}{u_{k}^{0}}\right),
\end{aligned}
$$

where repeated indices indicate summation, and $u_{a}^{N}$ is the mode $a$ group velocity in region $N$.

Figure 4-2 illustrates the effects of propagating four modes through $32 \mathrm{~km}$ of this idealized wave guide with one 200 meter width soliton at the $20 \mathrm{~km}$ point. The solid curves represent the expected mode coefficients or mode-filter outputs, $A_{1}(t), A_{2}(t)$, $A_{3}(t)$, and $A_{4}(t)$. The dotted curves are the individually scattered subarrivals. These individual subarrival pulses are not detectable in actual acoustic receptions. However, 


\section{Scattering from One Soliton}

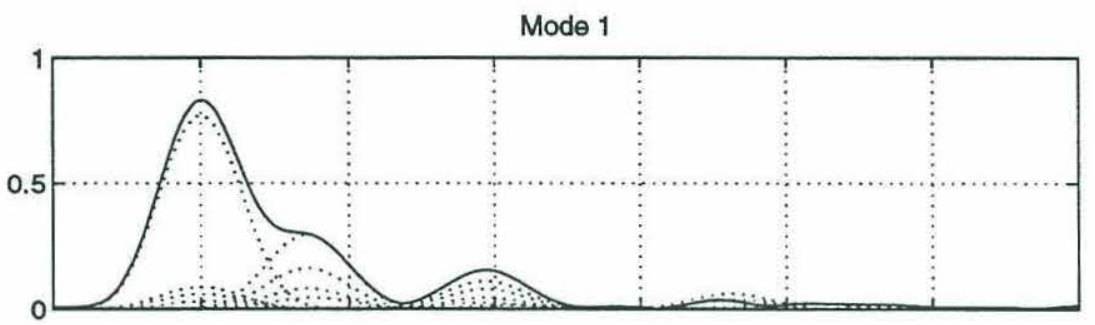

Mode 2

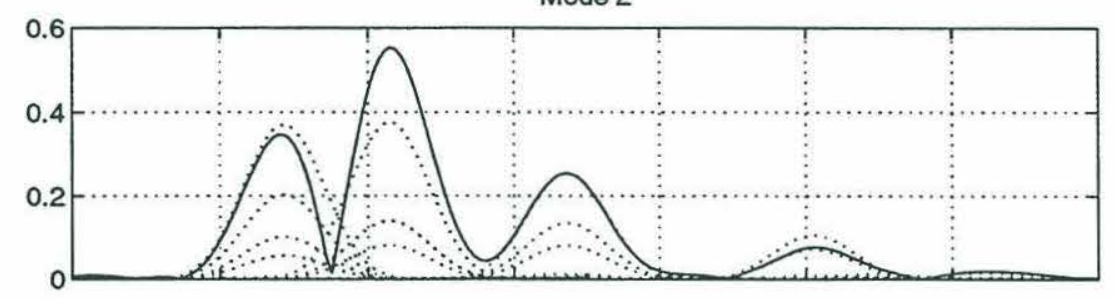

Mode 3

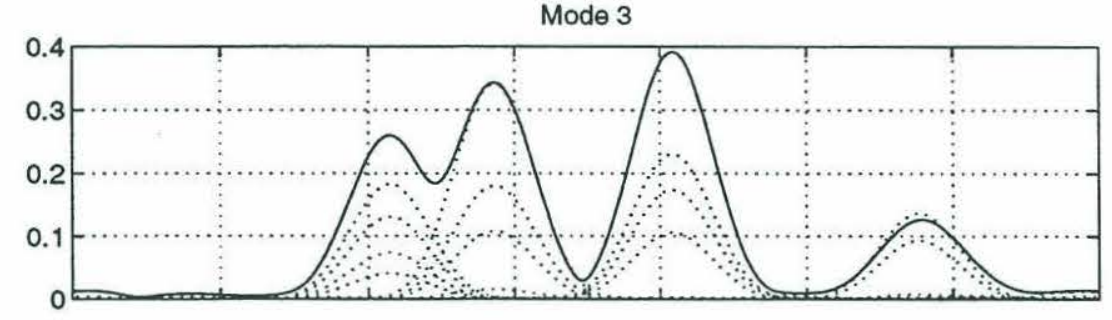

Mode 4

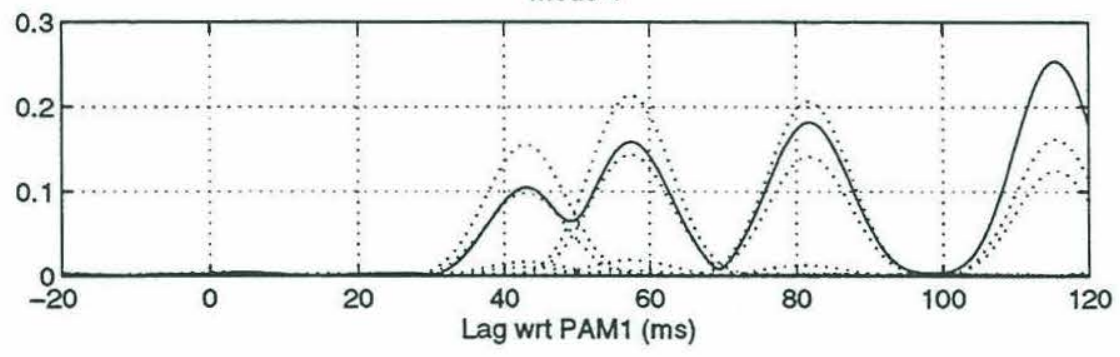

Figure 4-2: Propagation of four modes through $32 \mathrm{~km}$ of an idealized wave guide with one 200 meter soliton at the $20 \mathrm{~km}$ point. The solid curves are the absolute value of coherent sums of the $J^{2}=16$ dotted individually scattered arrivals. Starting amplitudes for mode 1 through 4 were $1.0,0.8,0.6$, and 0.4 respectively. 
as seen in Figure 4-2, we can sometimes infer, from the coherent sum, the relative levels of influence from some "general" paths through range-modenumber space.

Each of the mode 1-4 arrivals in Figure 4-2 shows influence from four different "general" paths through range-modenumber space. We call the first hump in the mode 1 arrival envelope a pseudo-adiabatic mode 1 (PAM1) arrival, as its peak arrives on or very near the time predicted for an adiabatically coupled mode 1 arrival. The general path for this PAM1 arrival hump could be referred to as "the 1:1 path" in this simple case, because all the energy traveled in mode 1 before reaching the soliton, took various paths through the soliton, and transited the remaining $11.8 \mathrm{~km}$ in mode 1. The energy in the second hump of the mode 1 arrival envelope (2:1 path) traveled the first $20 \mathrm{~km}$ in mode 2, took various paths through the soliton, and transited the remaining $11.8 \mathrm{~km}$ in mode 1 . Likewise, the energy in the first hump of the mode 2 arrival envelope (1:2 path) traveled the first $20 \mathrm{~km}$ in mode 1 , took various paths through the soliton, and transited the remaining $11.8 \mathrm{~km}$ in mode 2 . Similar "general" paths could be inferred from the remaining humps in the arrival envelopes.

The addition of a second soliton in the path increases the complication significantly (Figure 4-3); each mode arrival pattern now consists of the coherent sum of 256 $\left(=J^{2} \times J^{2}\right.$, where $\left.J=4\right)$ pulses that are shifted in phase, amplitude, and time. The arrival envelopes have more humps and inferences about their general paths are more difficult to make. The first humps in the mode 1-4 envelopes might be reasonably labeled 1:1:1, 1:1:2, 1:1:3, and 1:1:4 respectively, and their last humps 4:4:1, 4:4:2, 4:4:3, and 4:4:4 respectively, but with the interior it becomes more difficult. One could consider 16 possible general paths for each envelope, but the near-symmetry of the soliton placement in the waveguide would create very little separation between some of them (e.g. the 1:2:1 and 2:1:1 paths in the mode 1 arrival envelope).

Most of the humps do not correspond to any particular general path. Instead, they are the result of interference patterns between two or more general paths. This interference actually occurs to a greater or lesser extent in all the humps. The 4:4:4 path 


\section{Scattering from Two Solitons}
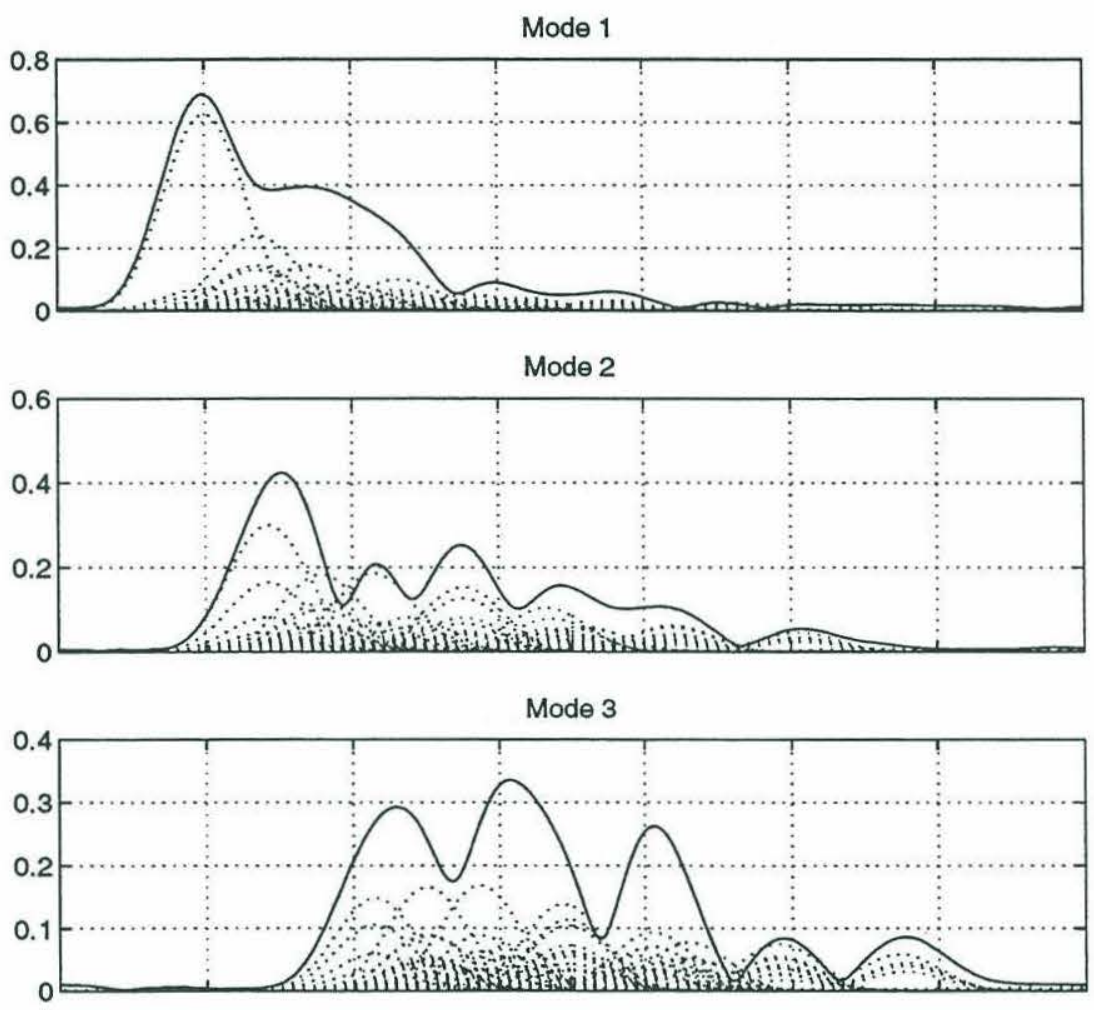

Mode 4

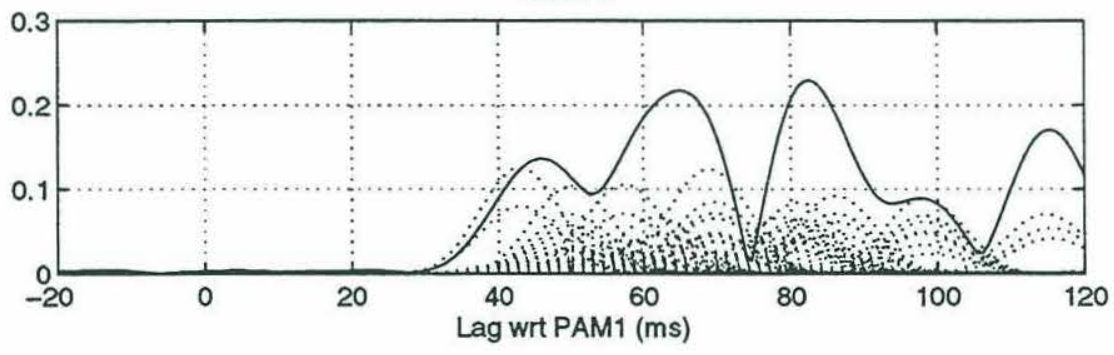

Figure 4-3: Propagation of four modes through $32 \mathrm{~km}$ of an idealized wave guide with 200 meter solitons at the 10 and $20 \mathrm{~km}$ points. The solid curves are the coherent sums of the $N^{2} \times N^{2}=256$ dotted individually scattered arrivals. Initial mode 1 through 4 amplitudes were $1.0,0.8,0.6$, and 0.4 respectively. 
(i.e. the PAM4 arrival), for example, shows little interference, while the interference in the neighborhood of the 1:1:3 path is significant.

The total summation approach can provide some valuable insight when dealing with one or two solitons, but it is quickly swamped by the exponential growth in subarrival pulses for multiple solitons in the path between the source and receiver (e.g. 65,536 pulses per mode with four modes and four solitons).

\subsubsection{Step Summation}

A computationally tractable alternative to the above method is to sum the pulses for each mode after each coupling event and create a new pulse shape that can be propagated to the next step. The new pulse shape for step $n$ is given by

$$
\tilde{p}_{a}^{n}(t)=C_{a b} \times \exp \left[i k_{r b}^{n-1}\left(r^{n}-r^{n-1}\right)\right] \times \tilde{p}_{b}^{n-1}\left(t+\frac{\left(r^{n}-r^{n-1}\right)}{u_{1}^{n-1}}-\frac{\left(r^{n}-r^{n-1}\right)}{u_{b}^{n-1}}\right) .
$$

The time shifts are again tracked as lag with respect to the PAM1 arrival. These time-shifts are subject to round-off error, if the step size is too small.

The step summation efficiently handles numerous sequential coupling events. Figure 4-4 illustrates temporal variations in mode propagation through 7 solitons as the solitons progress in lock-step $(0.8 \mathrm{~m} / \mathrm{s})$ up the waveguide. Even though lock-step propagation is unrealistic, it is shown as a type of lower bound on the variation in mode arrivals caused by soliton propagation. Actual solitons have phase speeds that are inherently variable, and they can propagate in offset directions that cause dramatic increases in apparent phase speed. A pattern of propagation with varying phase speeds causes even more variation in the arrival patterns, but it is not clear as to how much variation in phase speed would constitute a "more realistic" environment. Even with 7 solitons, the PAM1 arrival is still a dominant feature in many of the realizations.

These simulated arrivals show time-spreading and distortion levels that are quite 


\section{Scattering from Seven Solitons}
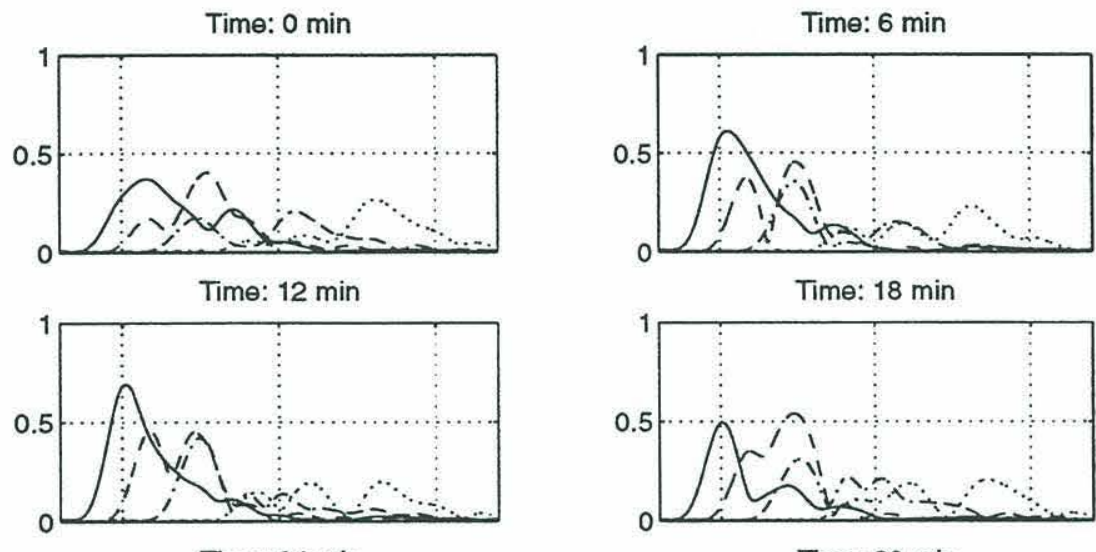

Time: $18 \mathrm{~min}$

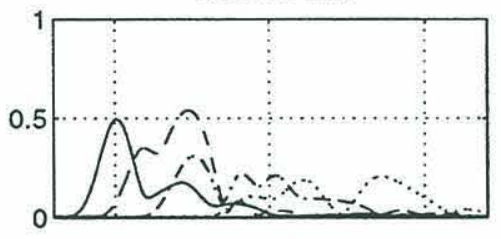

Time: $24 \mathrm{~min}$

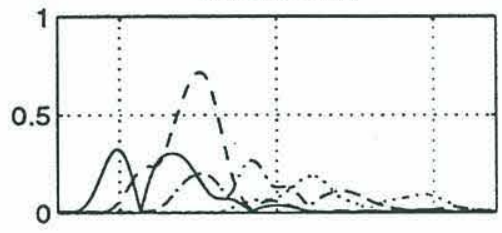

Time: $30 \mathrm{~min}$

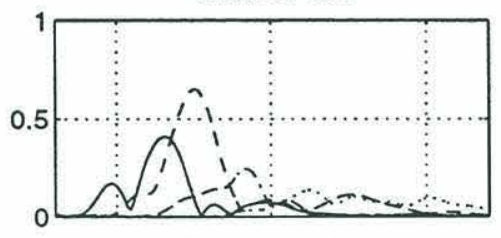

Time: $36 \mathrm{~min}$

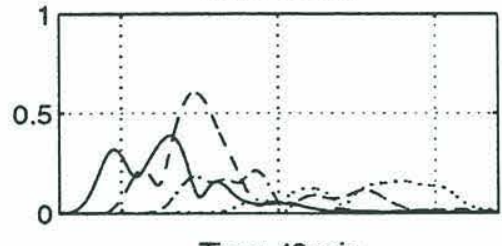

Time: $42 \mathrm{~min}$
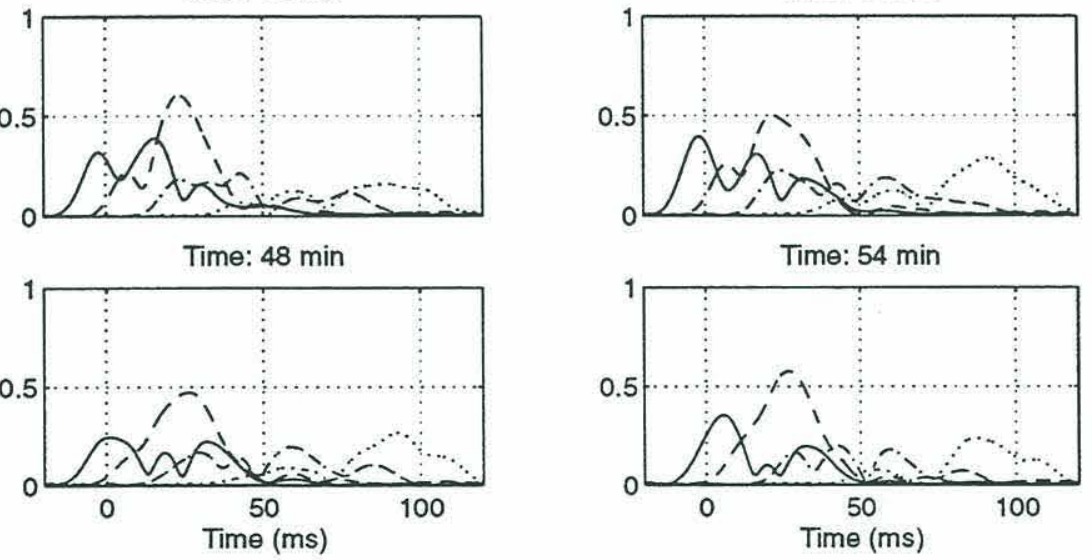

Figure 4-4: Simulated mode coefficient outputs, $A_{1}$ (solid), $A_{2}$ (dashed), $A_{3}$ (dot-dash), and $A_{4}$ (dotted), for propagation through seven idealized solitons. The solitons propagated toward the receiver in lock step at $0.8 \mathrm{~m} / \mathrm{s}$. The ten plots depict the arrival structures for sequential realizations at six minute intervals. 


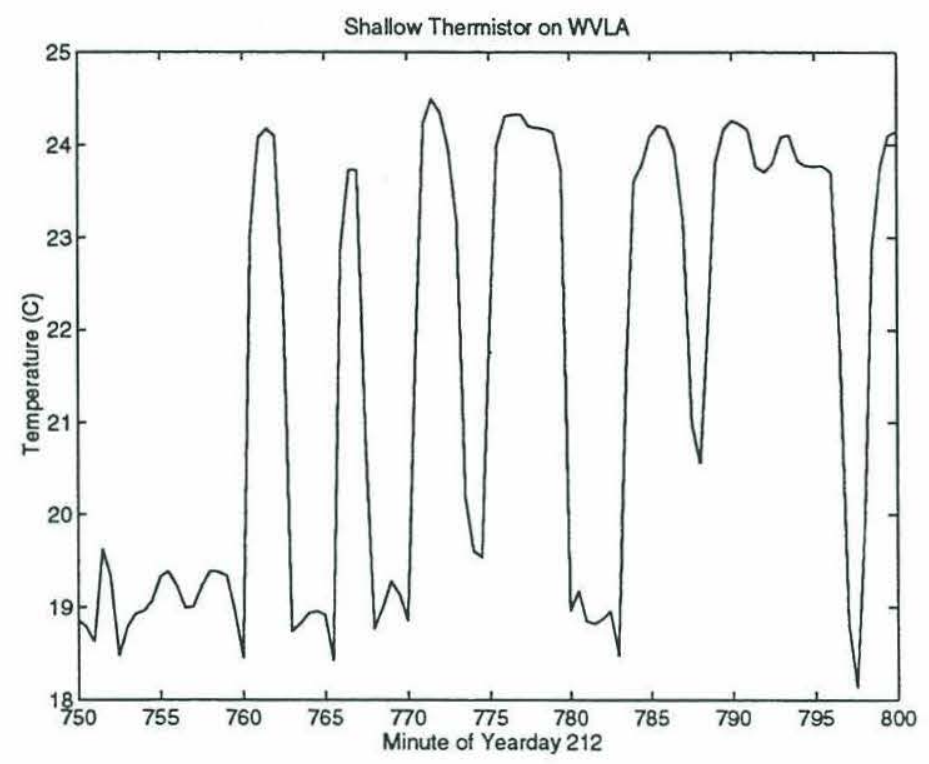

Figure 4-5: Time-series of temperature at a depth of 12.5 meters on the WVLA.

similar to the mode-filtered outputs of Figure 3-4.

\subsection{Incorporation of Field Measured SSPs}

The WVLA was instrumented with six thermistors that recorded temperature samples every 30 seconds. This record is principally used to create time-dependent mode filters that are used to analyze the acoustic arrivals at the array, but it is also a convenient source of realistic SSPs for use in the pulse propagation model.

Solitons influence the SSP through almost the entire water column, but the largest temperature fluctuation signals occur at depths within the main thermocline. Figure 4-5 shows 50 minutes of temperature data from the shallowest $(12.5 \mathrm{~m})$ thermistor on the array. The main thermocline for SWARM usually has a top edge at about 8 to $10 \mathrm{~m}$ depth, so the location of this thermistor is well suited for the detection of solitons; the SSPs from minute 777 and minute 781 were chosen as representative of profiles with and without a soliton. These SSPs, including extrapolations above $12.5 \mathrm{~m}$ and below $60.5 \mathrm{~m}$, are shown in Figure 4-6. The iso-velocity model utilized 


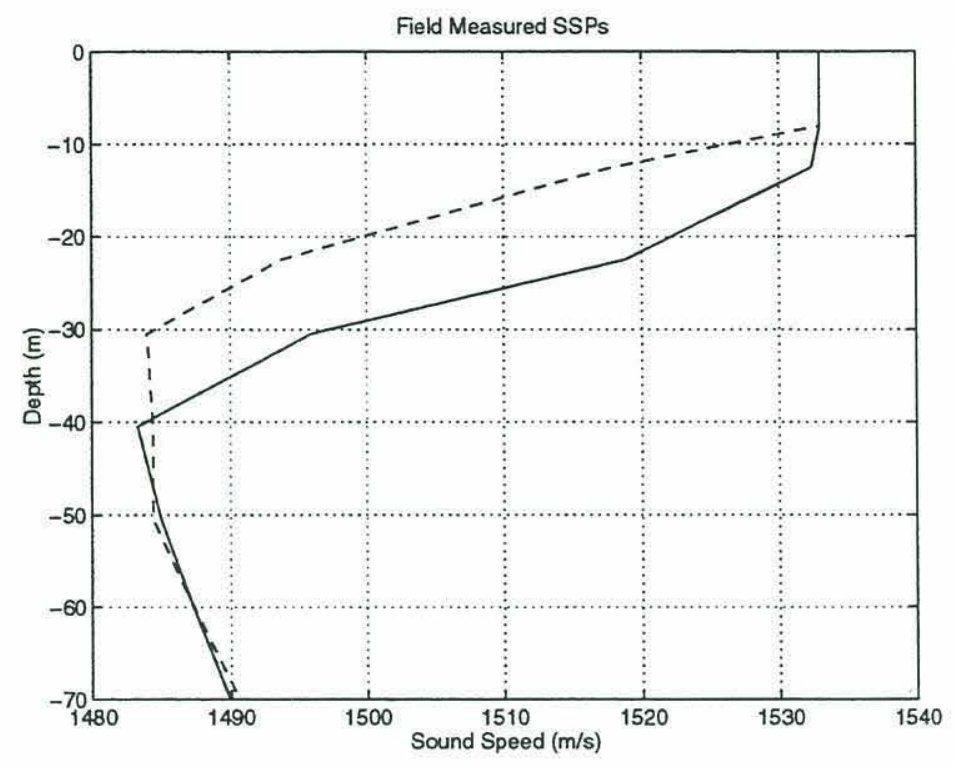

Figure 4-6: Field measured SSPs for use in the pulse-propagation model. The dashed line is representative of a standard SSP with no soliton. The solid line represents the soliton SSP.

a pressure release bottom for simplicity. The field measured SSPs were coupled to a bottom that is based on a sedimentary analysis in the vicinity of the WVLA [1]. The bottom has a sound speed that increases linearly from 1500 to $1800 \mathrm{~m} / \mathrm{s}$ in a $30 \mathrm{~m}$, $1.8 \mathrm{~g} / \mathrm{cm}^{3}$ density, sub layer over a rigid bottom. The best estimate of compressional wave attenuation in this sublayer is $0.092 \mathrm{~dB} / \mathrm{m}$ at $400 \mathrm{~Hz}$ (Altan Turgut, NRL, Washington, D.C., private communication), but as later results will indicate, this number is either too large by at least a factor of four or, more likely, is not applicable for the majority of the actual SWARM waveguide.

These SSPs should provide the model with mode dispersion characteristics that are closer to reality than the iso-velocity SSP used previously, but they also bring to light another question about the narrowband approximation of the model. 


\subsubsection{Validity of $400 \mathrm{~Hz}$ Narrowband Models for a Broad- band Pulse}

Working with an iso-velocity water profile and a pressure release bottom provides several advantages, including the fact that the sinusoidal mode shapes are frequency independent. As a result, the mode group speeds are also frequency independent. The simple pulse propagation model previously discussed used a narrowband approximation in the determination of coupling matrices and group velocities. The validity of this approximation becomes a significant issue with the introduction of realistic SSPs to the model.

Figure 4-7 compares soliton present and soliton absent mode shapes at the extremes of the pulse frequency domain $(355-445 \mathrm{~Hz})$. The mode shapes for these SWARM SSPs have a frequency dependence that appears minimal in modes 1 and 2, but it becomes somewhat more visible in modes 3 and 4 . The mode shape differences for the higher modes will contribute to some frequency dependence in the coupling matrices, particularly coupling in and out of mode 4 . This is not considered in the narrowband pulse propagation model; the pulse is assumed to travel and couple in accordance with the $400 \mathrm{~Hz}$ mode shapes.

Mode shape frequency dependence also leads to frequency dependence in group velocity. The group velocity is defined as

$$
u_{n}(\omega)=\frac{d \omega}{d k_{r n}} .
$$

Jensen et al. [13], using perturbation theory, derive an equation for the reciprocal of the group velocity,

$$
\frac{d k_{r}}{d \omega}=\frac{\omega}{k_{r}} \int_{0}^{D} \frac{\phi^{2}(z)}{\rho(z) c^{2}(z)} d z,
$$

that illustrates the dependence of group velocity on mode shape. Figure 4-8 shows the degree of frequency dependence in phase and group speed for the first four modes of the standard field measured SSP. Pulse distortion from frequency dispersion in the 


\section{Mode Shape Frequency Dependence}
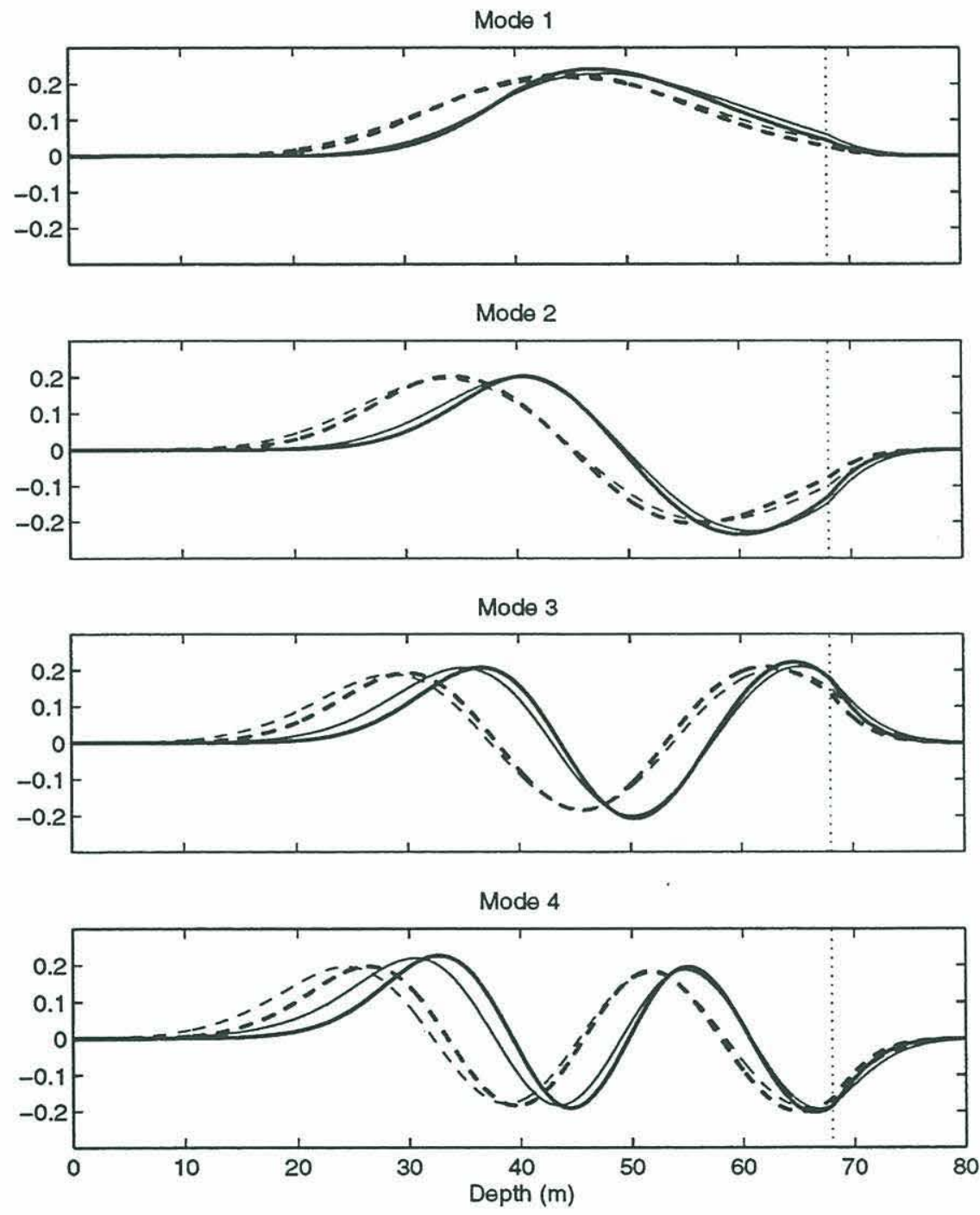

Figure 4-7: Mode shapes for field measured (SWARM) SSPs. The dashed lines are the $355 \mathrm{~Hz}$ (thin lines) and $445 \mathrm{~Hz}$ (thick lines) mode shapes corresponding to the standard SSP with no soliton. The solid lines are the same modes for the soliton SSP. The dotted line at $68 \mathrm{~m}$ indicates the sediment interface. 


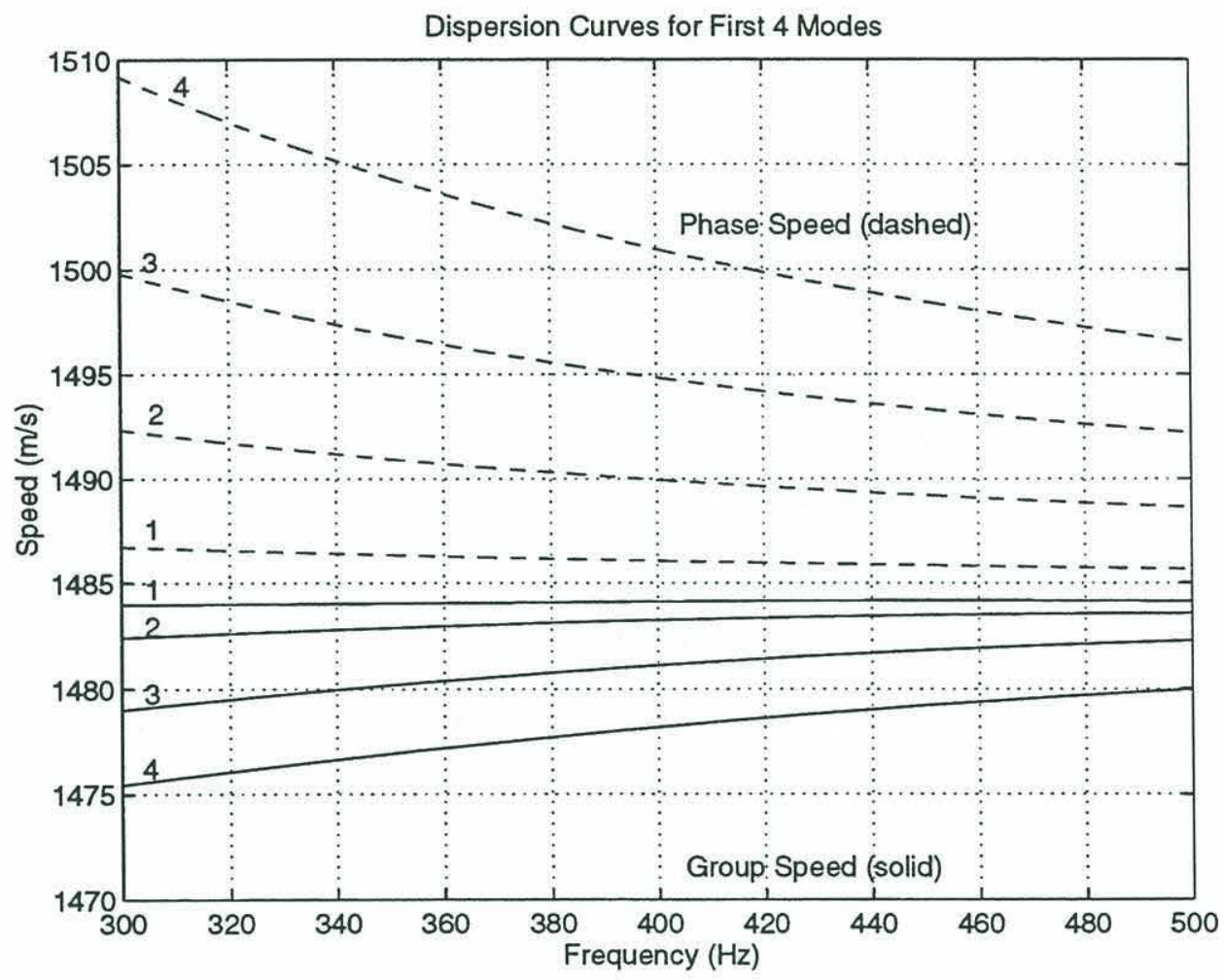

Figure 4-8: Phase and group speeds for the standard field measured SSP. Mode 1 has the lowest phase speed and the highest group speed. The remaining phase speeds increase monotonically with mode number, and the group speeds decrease monotonically with mode number. 
higher modes will clearly occur; the present time-domain model does not replicate this.

The switch from a time-domain propagation model to the frequency domain is fairly straight forward. Instead of simply propagating the replica correlated pulse shape, $\tilde{p}(t)$, at the $400 \mathrm{~Hz}$ modal phase and group speeds, allow its individual Fourier components $P(\omega)$, to propagate prior to synthesis, the resulting transmitted pulse shape,

$$
\tilde{p}_{n}(r, t)=\frac{1}{2 \pi} \int P(\omega) e^{i \omega t} e^{-i k_{r n}(\omega) r} d \omega,
$$

will show the spreading and intensity loss that is due to frequency dispersion. The synthesis results for different modes will reflect the differences in group speed between modes through the frequency dependence of the horizontal wavenumbers, $k_{r n}(\omega)$. The narrowband SIA coupling matrices used in the time domain model are linear operators that apply equally in the frequency domain. Figure 4-9 illustrates both the separating effects of mode dispersion and the pulse distortion effects of frequency dispersion that might be expected for individual mode pulses that travel the entire 32 $\mathrm{km}$ waveguide without any soliton scattering. An ideal frequency domain propagation model would also include frequency dependence in the coupling matrices, and in fairly simple waveguides, this is a possibility (e.g. the KRAKEN [12] field synthesis method, described in Appendix B), but, as also shown in Appendix B, the effects are minimal and the added complexity and computation time is not warranted.

\subsubsection{Adding Modal Attenuation}

As mentioned earlier, one of the estimated properties for the field measured SSPs was a compressional wave attenuation of $0.092 \mathrm{~dB} / \mathrm{m}$ in the bottom. This causes significant attenuation of the higher modes that interact with the bottom. The attenuation can be modeled through an imaginary term, $i \alpha$, added to the horizontal wave number, $k_{r}$. The KRAKEN [12] calculated $400 \mathrm{~Hz}$ mode attenuation levels for the SWARM SSPs are given in Table 4-1. The presence of the soliton depresses the SSP significantly, 

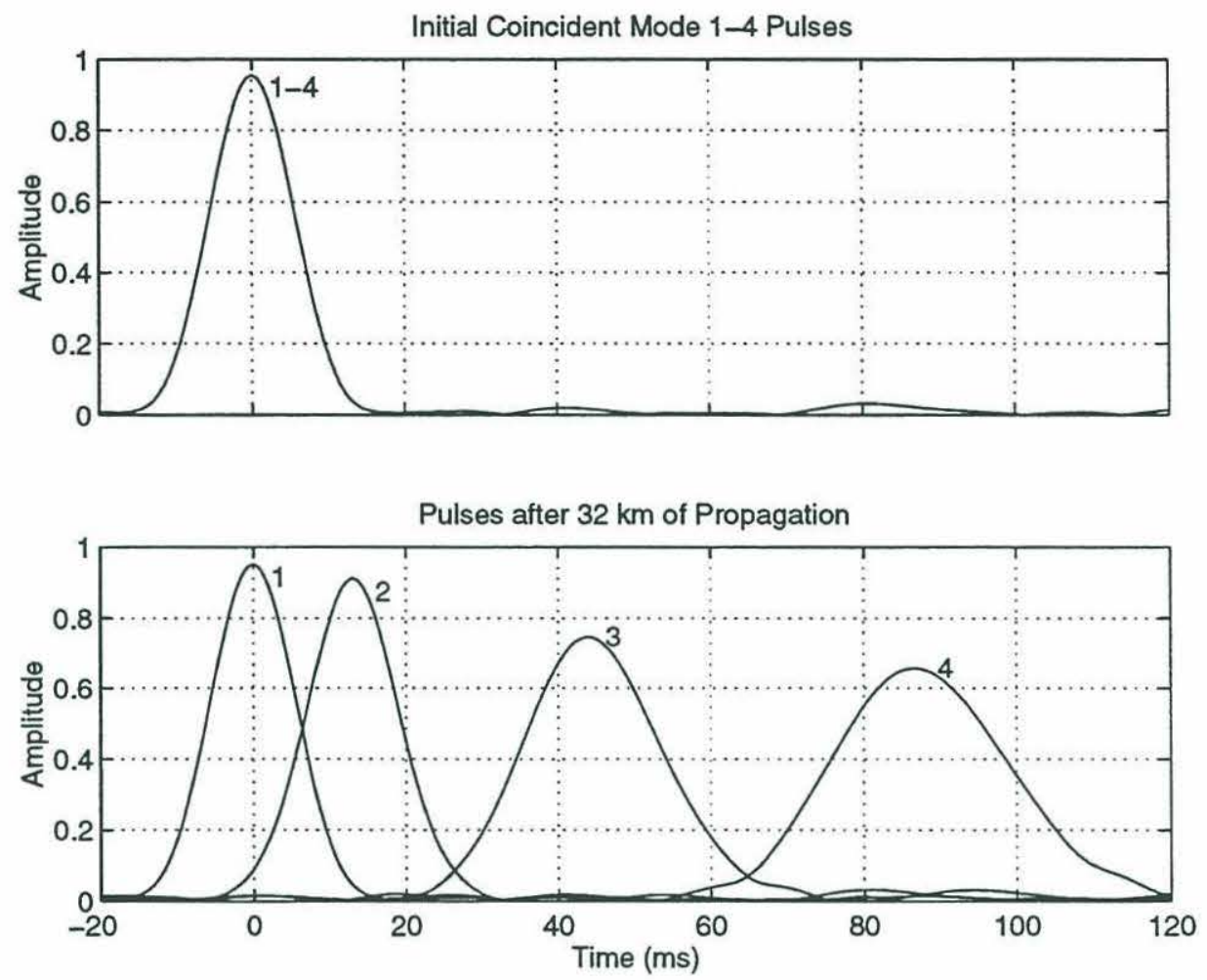

Figure 4-9: Effects of mode and frequency dispersion on single mode transmissions over $32 \mathrm{~km}$. The transmissions include no soliton scattering or intrinsic mode attenuation. The attenuation in the higher modes is due solely to frequency dispersion.

\begin{tabular}{|c|c|c|}
\hline \multirow{2}{*}{ Mode } & \multicolumn{2}{|c|}{ Attenuation $\left(\mathrm{km}^{-1}\right)$} \\
\cline { 2 - 3 } & Standard & Soliton \\
\hline 1 & 0.0094 & 0.0235 \\
2 & 0.0706 & 0.1782 \\
3 & 0.2042 & 0.3323 \\
4 & 0.3060 & 0.4090 \\
5 & 0.3751 & 0.5204 \\
6 & 0.4639 & 0.5547 \\
7 & 0.5465 & 0.5742 \\
8 & 0.7132 & 0.7457 \\
\hline
\end{tabular}

Table 4.1: Intrinsic mode attenuation parameters, $\gamma$, for various mode numbers. Values for both a soliton SSP and the standard field measured SSP without a soliton present are given. 

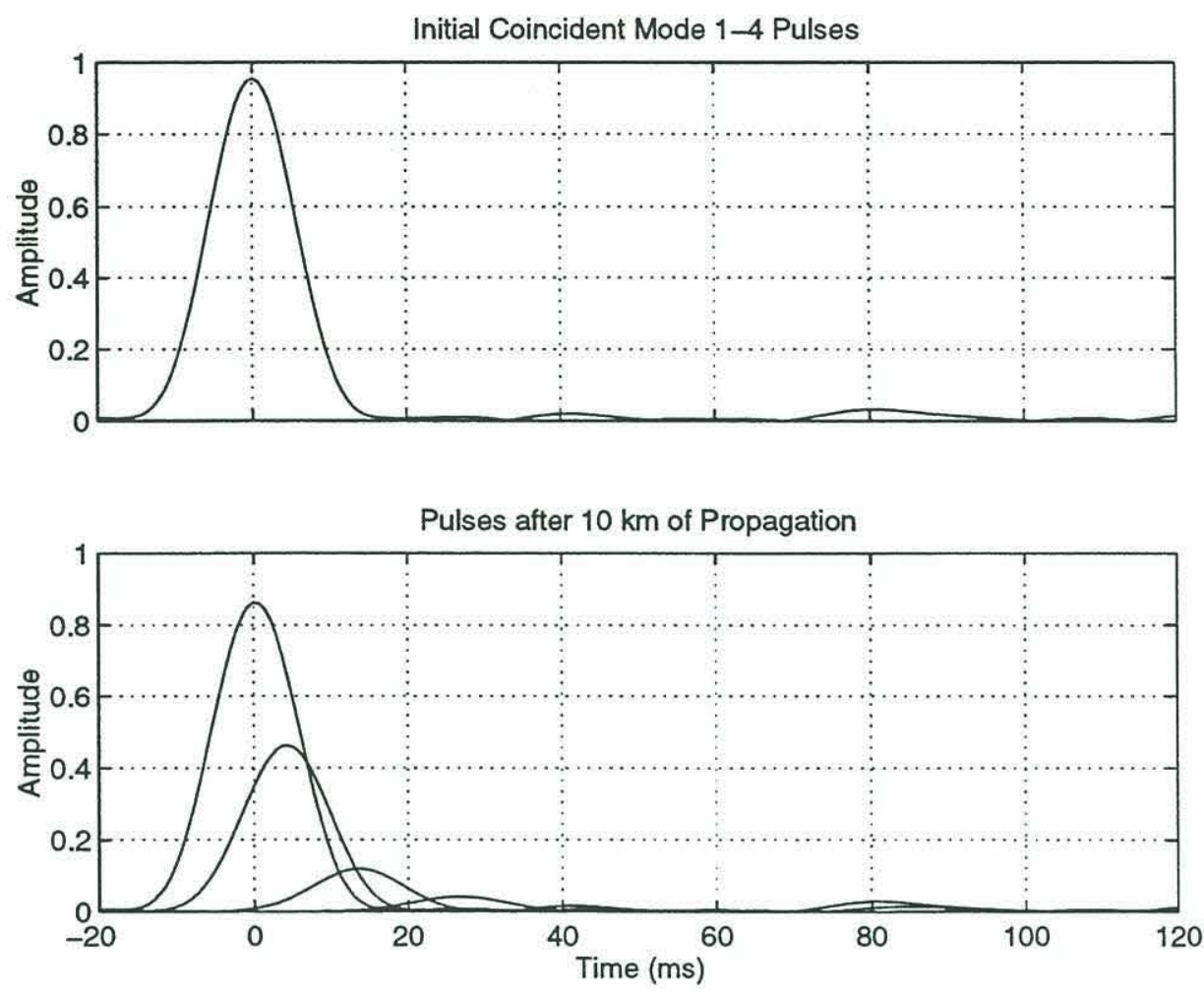

Figure 4-10: Effects of mode and frequency dispersion combined with intrinsic mode attenuation on single mode transmissions over $10 \mathrm{~km}$. The transmissions include no soliton scattering.

causing more bottom interaction and greater modal attenuation. Even the standard SSP attenuation levels are quite dramatic; figure 4-10 illustrates the effects of their application to a $10 \mathrm{~km}$ modal transmission. A field with an average number of solitons would have higher attenuation levels applied over about 10 percent of the range, causing even greater attenuation. These attenuation levels are probably too high, considering observed levels of time-spreading in actual data. If left at this level, the attenuation will all but eliminate modes 3 and 4 after a full $32 \mathrm{~km}$ transmission. As will be shown in Chapter 5, an "effective range averaged" compressional wave attenuation of $0.0115 \mathrm{~dB} / \mathrm{m}$ is more appropriate. 


\subsubsection{The Effects of Bottom Topography}

Waveguides with little horizontal variation in water column properties may still experience mode coupling, if there is significant variability in bottom topography. For soliton filled waveguides, the coupling caused by bottom topography produces a baseline level of scattering upon which is added time-varying volume (e.g. soliton) scattering. Given the bottom topography of the SWARM waveguide (see Figure 2-1), some noticeable level of baseline scattering is expected.

The simulations shown in Figure 4-11, which used a range dependent waveguide based on the bottom topography of Figure 2-1, can provide a good estimate of this baseline. The range independent SSP used in the "No Ducting" case is a modified version the "non-soliton" SSP of Figure 4-6. The modification consists of imposing the $50.5 \mathrm{~m}$ temperature from $50.5 \mathrm{~m}$ to the bottom, producing a "worst case" scenario for mode 1 scattering. The "Ducting" SSP is the same, except the $60.5 \mathrm{~m}$ temperature is imposed from $50.5 \mathrm{~m}$ to the bottom depth, noticeably reducing the topographic coupling effects for the lower modes. The thinner lines in this figure represent predicted arrival shapes for modes that propagate adiabatically (i.e. no scattering of energy between modes).

\subsection{Incorporation of a Background Internal Wave Spectrum}

Although the primary modeling concern in this paper is internal wave solitons, linear internal wave fields, which are also present in shallow water, should not be neglected. They are usually modeled and studied as a stochastic process that produces random sound-speed fluctuations, $\delta c(r, z, t)$, about a background profile, $c_{0}(z)$. A "standard method" of producing these random sound speed fluctuations and resultant coupling matrices in deep water was developed by Dozier and Tappert [14]; it generally consists of using a GM spectrum as the basis for creating a random realization of the 


\section{Coupling from Bottom Topography}
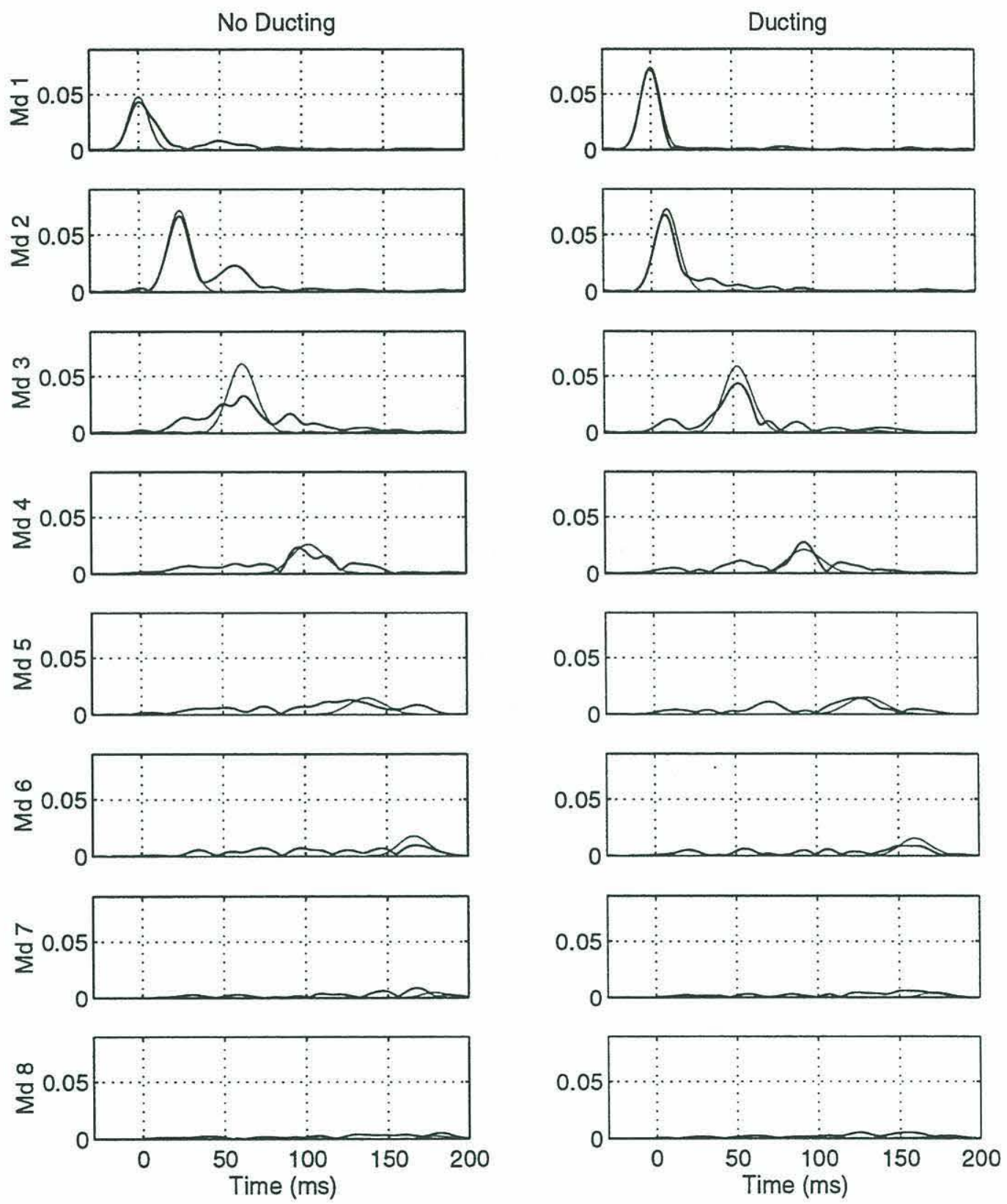

Figure 4-11: Mode coupling effects of the bottom topography shown in Figure 2-1 when the SSP is a modified version the "non-soliton" SSP of Figure 4-6. The "No Ducting" modification consists of imposing the $50.5 \mathrm{~m}$ temperature from $50.5 \mathrm{~m}$ to the bottom depth, and the "Ducting" modification consists of imposing the 60.5 $\mathrm{m}$ temperature from $50.5 \mathrm{~m}$ to the bottom depth. The thinner lines in this figure represent predicted arrival shapes for modes that propagate adiabatically. 
background internal wavefield, $\zeta(r, z, t)$, which is proportional to $\delta c(r, z, t)$. The resulting range and depth averaged $\left\langle\left(\delta c / c_{0}\right)^{2}\right\rangle$ value of the field controls the rate at which energy is transferred between modes. The amount of energy transferred between modes is estimated by sampling $\delta c(r, z, t)$ and computing coupling matrices at a fairly coarse interval (Dozier and Tappert [15] use $1 \mathrm{~km}$ ). The results are then averaged over numerous realizations.

It is unclear as to how close a GM spectrum approximates shallow water internal waves. Lynch et al. [16] present a spectral analysis of data from a thermistor string in $400 \mathrm{~m}$ of water that is consistent with a GM spectrum at frequencies above about 8 cpd, but the spectrum in the 3 to $8 \mathrm{cpd}$ region is significantly less energetic relative to GM. This single string could not provide any information concerning the directional spectrum in the region.

In the absence of any other data to the contrary, the GM spectrum assumption provides a method of developing somewhat realistic levels of vertical and horizontal coherence. The vertical coherence is critical to the calculation of the coupling matrix at a given range and the horizontal coherence provides some realism to the range dependence of these matrices. On the other hand, since the GM spectrum may not really be representative of shallow water, the modeling of correlation functions through a rather complicated GM internal wave synthesis may not be the best approach.

\subsubsection{EOF Soundspeed Perturbation Modes}

Empirical orthogonal function (EOF) modes of thermistor string temperature perturbations are a good alternative to internal wave synthesis. Unlike the GM synthesis approximations, the EOF modes will include influence from both linear and nonlinear internal waves, so the EOF modes can be used as stand alone random realizations. The range and depth averaged $\left\langle\left(\delta c / c_{0}\right)^{2}\right\rangle$ value of an EOF realization is easily scaled up or down. When scaled down it can be used in conjunction with SIA solitons as a background field, providing additional scattering between SIA solitons. 
The main limitation associated with this or any other model that includes Dozier and Tappert [14] coupling is that the results are only valid in a stochastic sense. Dozier and Tappert [15] restrict their own results to averages over a hundred random realizations. The Dozier and Tappert [14] computations and several issues concerning the implementation of EOF fields are discussed in more detail in Appendix C.

\subsection{Fine Scale Coupled Mode Computations}

The large step size employed with Dozier and Tappert [14] coupling is the primary impediment to achieving a deterministic mode arrival model, but as shown in Appendix $\mathrm{C}$, even when implemented with fine scale sampling, they are still not reliable predictors of deterministic mode arrival patterns.

A more accurate and workable fine scale, range dependent, frequency domain, coupled mode model can be developed using the equation (4.2) narrowband coupling matrix. The range dependence of the model is determined by "propagating", with-

out dispersion, the SSPs measured at the WVLA towards the source at $0.8 \mathrm{~m} / \mathrm{s}$. Sampling the WVLA SSPs every minute yields a $48 \mathrm{~m}$ step size for the "propagated thermistor string" waveguide; a series of coupling matrices can then be calculated for the transitions between the SSPs.

Equation (4.2), as it stands, works well enough for the limited numbers of coupling events required with SIA solitons, but it requires modification if this finer scale (48 $\mathrm{m}$ ) range dependence is to be employed. Ideally a numerical computation of equation (4.2) using the same SSP on both sides would yield,

$$
C=\frac{1}{2} \int \frac{\phi(z) \phi(z)}{\rho(z)} d z+\frac{k}{2 k} \int \frac{\phi(z) \phi(z)}{\rho(z)} d z=I
$$

an identity matrix, but the six decimal place, 1001 point, ASCII mode shapes provided 
by the KRAKEN [12] plotting routine "plotmodeM" yields

$$
\int \frac{\phi_{n}(z) \phi_{n}(z)}{\rho(z)} \neq 1
$$

The integrals are very close to one, typically around 0.9996 for the higher modes, but the cumulative effect over hundreds of coupling events is noticeable. The numerical implementation of equation (4.2) also produces small (4th decimal place) erroneous off diagonal terms.

All these small error sources can be readily eliminated for the trivial case of coupling into an identical environment by multiplying the equation (4.2) coupling matrix by its inverse, creating,

$$
C^{\prime}=C^{-1} C=I,
$$

the desired identity matrix. In a fine scale implementation, the coupling matrices are non-trivial, but the differences between SSPs in adjacent regions are small. This suggests a corrected coupling matrix,

$$
C_{12}^{\prime}=C_{22}^{-1} C_{12}
$$

Kuperman et al. [17] deal with a truncated coupling coefficient integral in a similar fashion. The correction matrix, $C_{22}^{-1}$, is always close to an identity matrix, insignificant over a small number coupling calculations, but it can be important when small range steps are used over large distances. Consider a $32 \mathrm{~km}$ waveguide with a $48 \mathrm{~m}$ step size. The 670 coupling matrices it requires will generate significant cumulative errors from small individual errors in coupling coefficients (e.g. .9996 $6^{670}=.765$ ).

The effectiveness of this correction factor can be seen in the close fit of the mode arrival patterns in Figure 4-12. Both sets of patterns were generated using the same $32 \mathrm{~km}$ waveguide, but one used a $48 \mathrm{~m}$ step size (dotted), and the other employed 

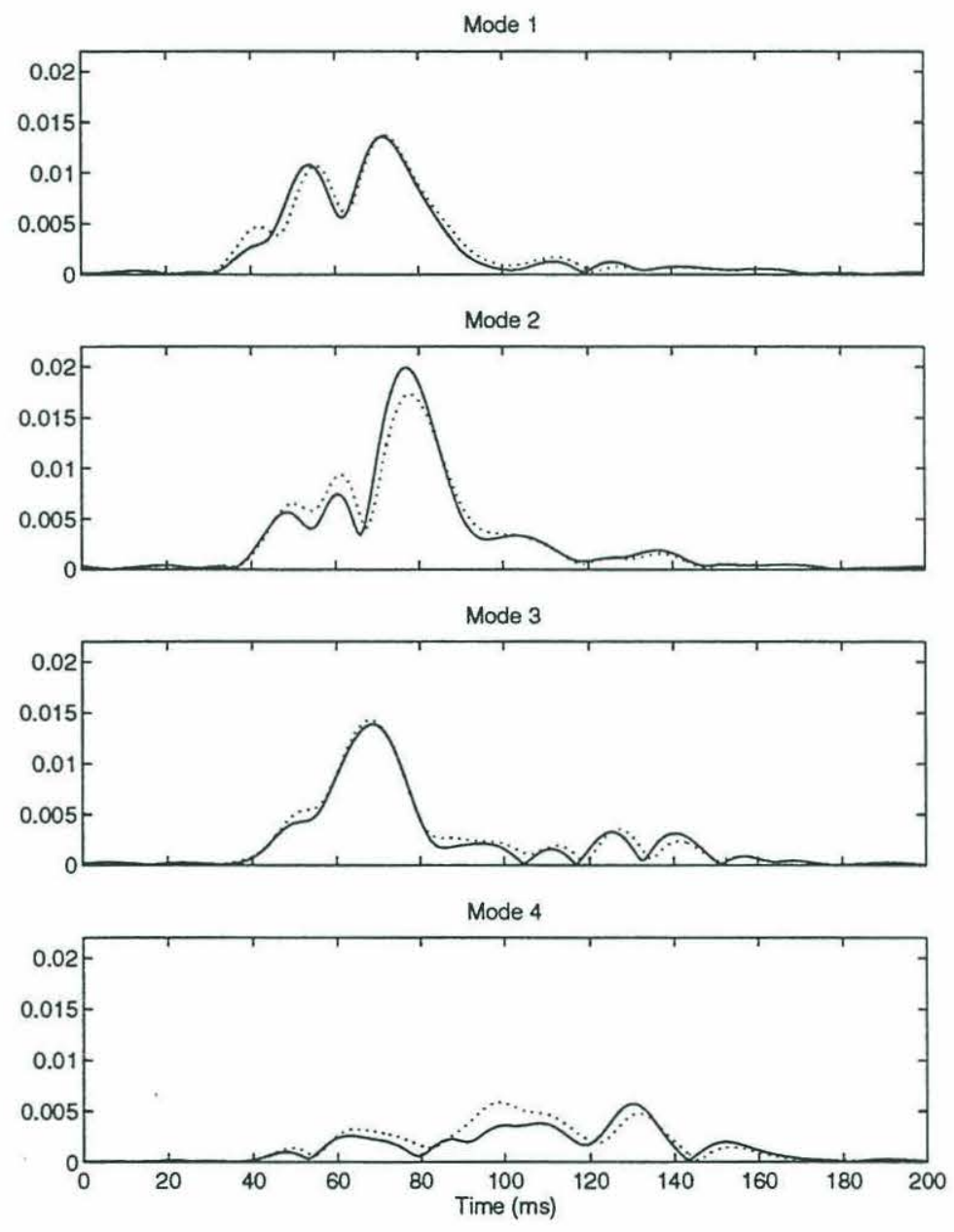

Figure 4-12: Comparison of fine scale coupled mode arrivals using $24 \mathrm{~m}$ (solid) and $48 \mathrm{~m}$ (dotted) step sizes. The $32 \mathrm{~km}$ wave guide under evaluation was generated from range propagated WVLA thermistor records. 
a $24 \mathrm{~m}$ step size (solid), requiring twice as many coupling calculations. The $32 \mathrm{~km}$ waveguide is derived directly from range propagated WVLA thermistor records. The transformation from time dependence to range dependence is made by assuming all the temperature disturbances are propagating without dispersion at $0.8 \mathrm{~m} / \mathrm{s}$ along the same line as the acoustic transmission path. The reasoning behind this assumption is discussed in the horizontal EOF analysis section of Appendix C. Had the coupling matrices not been corrected properly, the smaller step size model, with 1340 coupling events, would have diverged noticeably from the $48 \mathrm{~m}$ step size model. The closeness of fit also indicates that the model is at or near solution convergence in terms of step size at the $48 \mathrm{~m}$ point.

\subsection{Putting it All Together}

Starting with a simple near-isovelocity narrowband SIA soliton coupling model, we have explored and considered various improvements on the basic model. We will now sharpen our focus and limit further analysis to two basic model types. The first model, emphasizing speed, has the following steps:

1. Create waveguides by alternating between two different SSPs, making solitons in the Preisig and Duda [2] SIA fashion. The SSPs, shown in Figure 4-6, are from the WVLA at periods with and without a soliton present.

2. Conduct mode propagation in the frequency domain, using interpolated, KRAKEN calculated, modal wavenumber and attenuation spectra over the bandwidth of the SWARM $400 \mathrm{~Hz}$ signal.

3. Use the narrowband approximation in coupling matrices.

This model will be used primarily for the purpose of analyzing the effects of solitons on mode 1 arrivals. The addition of bottom topography is feasible, but complicated, and, as shown in Figures 4-11 and 4-12, it would have a very limited impact on mode 
1 scattering. As discussed above, consideration was given to adding background scattering, in the form of EOF sound speed perturbations and Dozier and Tappert [14] coupling, to this SIA model. This would slow the model considerably and add an extra analysis dimension in the form of the background field strength. This added complexity will be rejected in favor of an even slower, but more straightforward, fine scale coupling method using range-propagated WVLA thermistor records. This second model will:

1. Use $32 \mathrm{~km}$ waveguides derived directly from range propagated WVLA thermistor records. The transformation from time dependence to range dependence is made by assuming all the temperature disturbances are propagating without dispersion at $0.8 \mathrm{~m} / \mathrm{s}$ along the same line as the acoustic transmission path. We will use a $48 \mathrm{~m}$ step size that corresponds conveniently to one minute of the thermistor record.

2. Conduct mode propagation in the frequency domain, using interpolated modal wavenumber and attenuation spectra over the bandwidth of the SWARM 400 $\mathrm{Hz}$ signal.

3. Use the narrowband approximation in coupling matrices.

This method implicitly includes the soundspeed perturbations from both linear and non-linear internal waves. The addition of bottom topography in this model is not feasible, because the modeled temporal variations in the waveguide rely on a range independent depth. 


\section{Chapter 5}

\section{Model Analysis}

\subsection{Monte Carlo Analysis of the SIA Model}

The mode arrival structures of actual data (Figures 3-4 and 3-5) are very complicated, so choosing relevant statistics to describe the shape or distortion of arrivals is not straightforward. The SIA numerical model described in the previous chapter is used to analyze the sensitivity of various measures of mode arrival distortion (e.g. spread and bias) to changes in the soliton field between the source and receiver.

\subsubsection{Relevant Statistics}

The main purpose of these simulation runs is to study the effects of varying the numbers and locations of solitons on the mode 1 arrival spread and bias. The spread is a measure of the width of the arrival pulse and the bias is a measure of the difference between the average arrival time of mode 1 energy and the PAM1 arrival time.

Measurement of spread and bias in an arrival is somewhat subjective; there are many similar mathematical measures that give slightly different results; the subjectivity comes from choosing which is "best". Some reasonable bias measurement candidates are the displacements of the peak, mean, and median arrival times of the mode 1 arrival envelopes relative to the PAM1 arrival time. There are several can- 


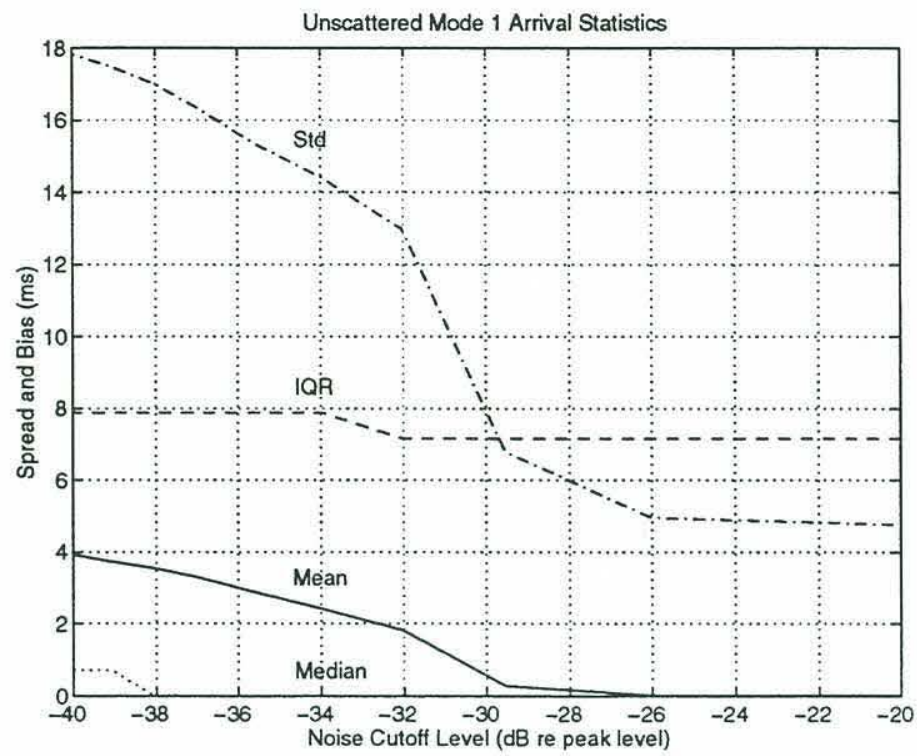

Figure 5-1: The mean (-), median $(\cdots)$, standard deviation (-•), and interquartile range (- -) of an unscattered mode 1 arrival plotted as a function of noise cutoff level. The median reaches its correct value of zero at a noise cutoff level of $-38 \mathrm{~dB}$ relative to the peak of the arrival.

didates for gauging the spread: the standard deviation of the arrival envelope; the interquartile range (the difference between the the 75 th and 25 th percentile of the arrival envelope); and the difference between the mean and peak arrival time.

Unfortunately, several of these commonly used statistical quantities can be corrupted by the trailing noisy side lobes of the replica correlation pulse (Figure 2-2). A clear picture of this corruption can be seen by computing the mean, median, standard deviation, and interquartile range (IQR) of the pulse shape in figure 2-2 as a function of noise cutoff level (Figure 5-1). At a noise cutoff of level of $40 \mathrm{~dB}$ below the peak, the sidelobe induced corruptions in the mean and standard deviation are about 4 and $13 \mathrm{~ms}$ respectively. The differences between initial ( $-40 \mathrm{~dB})$ level and asymptotic levels for the median and IQR are quite small in comparison. The median reaches its correct value of zero at a noise cutoff level of $-38 \mathrm{~dB}$, and the IQR reaches its asymptotic value at a cutoff of $-32 \mathrm{~dB}$. As the cutoff threshold is raised, all the errors go to zero, with the asymptotic levels in the standard deviation and IQR consistent 


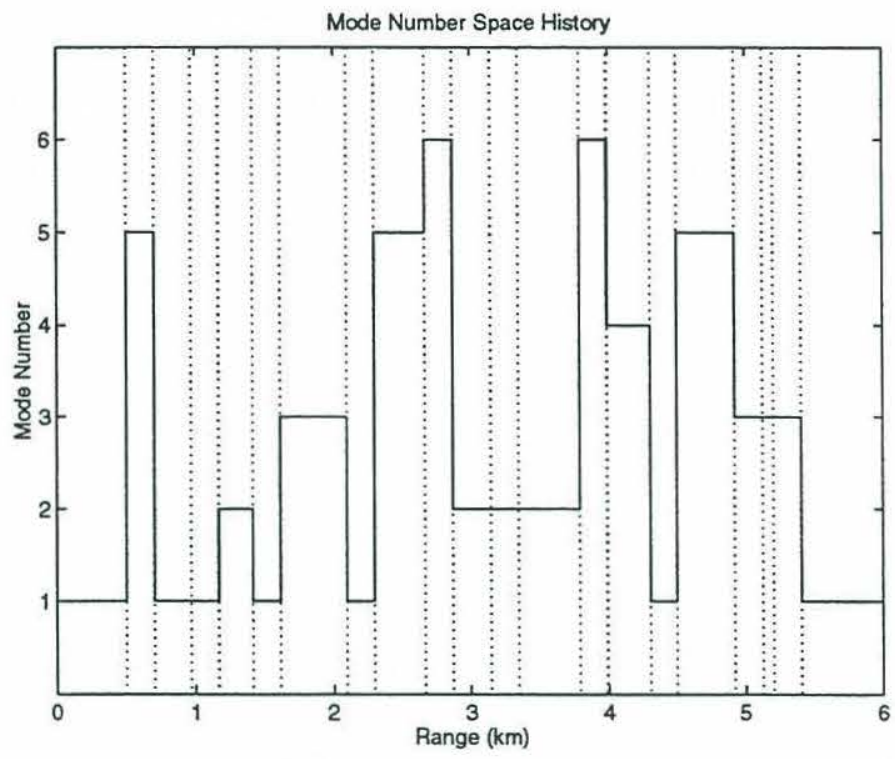

Figure 5-2: The solid line shows the mode number space history of one of the $6^{20}$ subarrivals that would make up the mode 1 arrival from a six mode transmission through the 10 SIA soliton waveguide that is indicated by the dotted lines.

with a gaussian pulse (IQR is about 1.35 times the standard deviation for a gaussian pulse). A noise cutoff at $-25 \mathrm{~dB}$ re peak was chosen as appropriate whenever the mean and standard deviations are to be used for spread and bias quantification; for consistency, it was used in calculations of the median and IQR as well.

The travel time statistics analyzed in this paper will, for the most part, be limited to the above described statistics, but this list is by no means exhaustive. Travel time statistics are not even limited to computations involving the time-domain mode arrival envelope. Colosi and Flatté [10], for example, estimate modal travel time by determining the average slope of modal phase as a function of frequency.

To some degree, the spread and bias of a mode 1 arrival depends on the wavenumber space (mode vs. range) history of each subarrival that makes up a given arrival. The solid line in Figure 5-2 shows the mode number space history of one of the $6^{20}$ subarrivals that would make up a mode one arrival in the 10 SIA soliton waveguide that is indicated by the dotted lines in the same figure. The phase relationships between the subarrivals are very important, but, on average, higher percentages of 
higher modes in the histories cause greater bias, and greater diversity in mode number histories of the subarrivals cause greater spread. In other words, on average, the bias in an arrival is determined by the mean modenumber history of the subarrivals and the spread of the arrival is determined by the variance of the modenumber histories of the subarrivals.

\subsubsection{Simulation Parameters}

Eight modes were propagated and scattered through the simulated waveguides that will be discussed in this section. The modeled waveguides support upwards of 40 propagating modes for the $400 \mathrm{~Hz}$ source, but the modes above 8 are of little consequence after about $5 \mathrm{~km}$. The most serious drawback of this truncated sum approach is exposed when one considers the problem of adequately modeling coupling events that occur within the first few kilometers of the source. The "near-source" coupling of energy from modes above 8 might reasonably affect the mode 1-8 arrivals at the receiver. However, trials using 6,8 , and 10 modes in the model show convergence for the mode 1-4 arrivals at the receiver when 8 modes are used in "near-source" coupling scenarios. The simple nature of the SIA model, as implemented, limits the number of other parameters open for adjustment. The "soliton" and "no soliton" SSPs are fixed by design; other parameters, like source depth and receiver range, are set to try to match the conditions in the actual experiment.

Another parameter we would like to match to the actual conditions at the SWARM site is bottom attenuation. Chirp sonar inversions for bottom parameters near the WVLA infer a sediment layer (specific gravity - 1.8) about $30 \mathrm{~m}$ deep, whose sound speed ranges from 1500 to $1800 \mathrm{~m} / \mathrm{s}$ [1]. Given these characteristics, Altan Turgut (NRL, Washington D.C., private communication) estimates the compressional wave attenuation in the bottom to be in the neighborhood of $.092 \mathrm{~dB} / \mathrm{m}$ at $400 \mathrm{~Hz}$. Although this level is on the low end of published attenuation level estimates for sediments with soundspeeds in the 1500 to $1800 \mathrm{~m} / \mathrm{s}$ range (e.g. clay, silt, sand, and 
gravel) [13], the resulting modal attenuation levels are still quite significant (see Figure 4-10).

Figure 5-3 shows six random realizations of the type of SIA soliton waveguides that will be referred to in the analysis that follows. Using a compressional wave attenuation of $.092 \mathrm{~dB} / \mathrm{m}$ in the modeled bottom of these waveguides yields predictions for mode 1 spread that are significantly below the values observed in actual arrivals (not shown), and while a compressional wave attenuation of $.046 \mathrm{~dB} / \mathrm{m}$ (half of the predicted value) provides simulation results that are somewhat better (Figure 5-4), they are still off the mark. A comparison of the simulated arrivals in Figure 5-4 with actual arrivals in Figure 3-4 illustrates this point. The spread is still not large enough, and the PAM1 arrival is much stronger than the average real data arrival. Reducing the compressional wave attenuation in the bottom to $0.023 \mathrm{~dB} / \mathrm{m}$ provides even more spread and dampens the PAM1 arrival (not shown), and finally, as shown in Figure 55 , using a bottom attenuation of $0.0115 \mathrm{~dB} / \mathrm{m}$ produces arrivals that are very similar to the outputs shown in Figures 3-4 and 3-5. Using these comparisons, an attenuation level of $0.0115 \mathrm{~dB} / \mathrm{m}$ appears to be the best choice.

The differences between results using $0.023 \mathrm{~dB} / \mathrm{m}$ and $0.0115 \mathrm{~dB} / \mathrm{m}$ are not large; limited Monte Carlo simulations using both $0.023 \mathrm{~dB} / \mathrm{m}$ and $0.0115 \mathrm{~dB} / \mathrm{m}$ were conducted, and the results were similar, but the average mode 1 arrival bias levels were about 20 percent with $0.023 \mathrm{~dB} / \mathrm{m}$. Part of the "required reduction" in the level of bottom attenuation stems from the range dependence of bottom characteristics; the sediment layer is reduced to less than $1 \mathrm{~m}$ in thickness over much of the path [1]. A reduction in the thickness of our simulated bottom to $1 \mathrm{~m}$ reduces modal attenuation levels by a factor of three. However, no attempt has been made to introduce range dependence into the bottom characteristics; the simulations shown in the next section were all calculated using the $30 \mathrm{~m}$ sediment with an "effective range averaged" compressional wave attenuation level of $0.0115 \mathrm{~dB} / \mathrm{m}$. 


\section{Random SIA Waveguides}

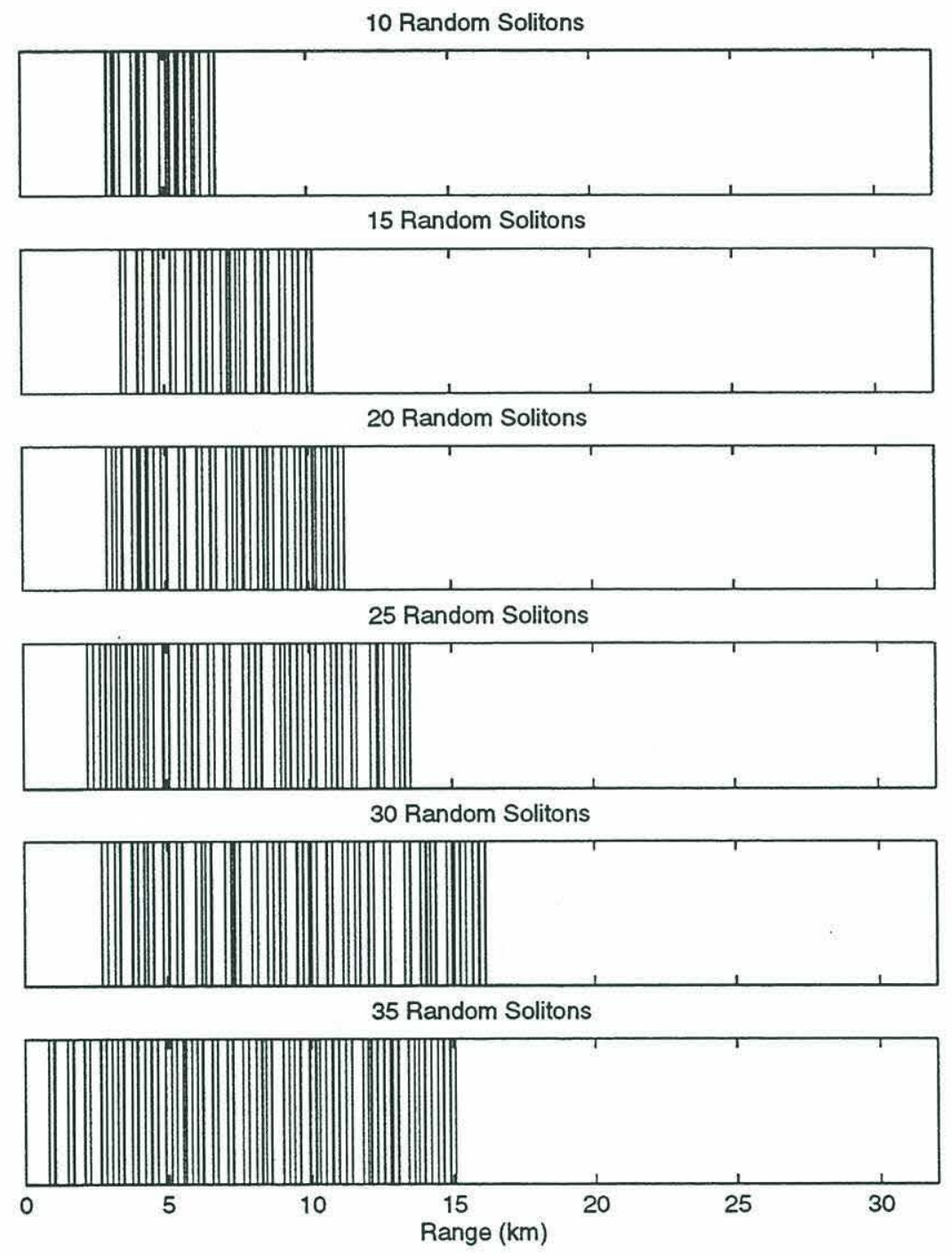

Figure 5-3: Random realizations of test waveguides with from 10 to $35200 \mathrm{~m}$ SIA solitons over $32 \mathrm{~km}$. The first soliton is placed randomly about $2.5 \mathrm{~km}$ from the source, and the remainder are added randomly from there, with an average of $450 \mathrm{~m}$ between leading edges. 

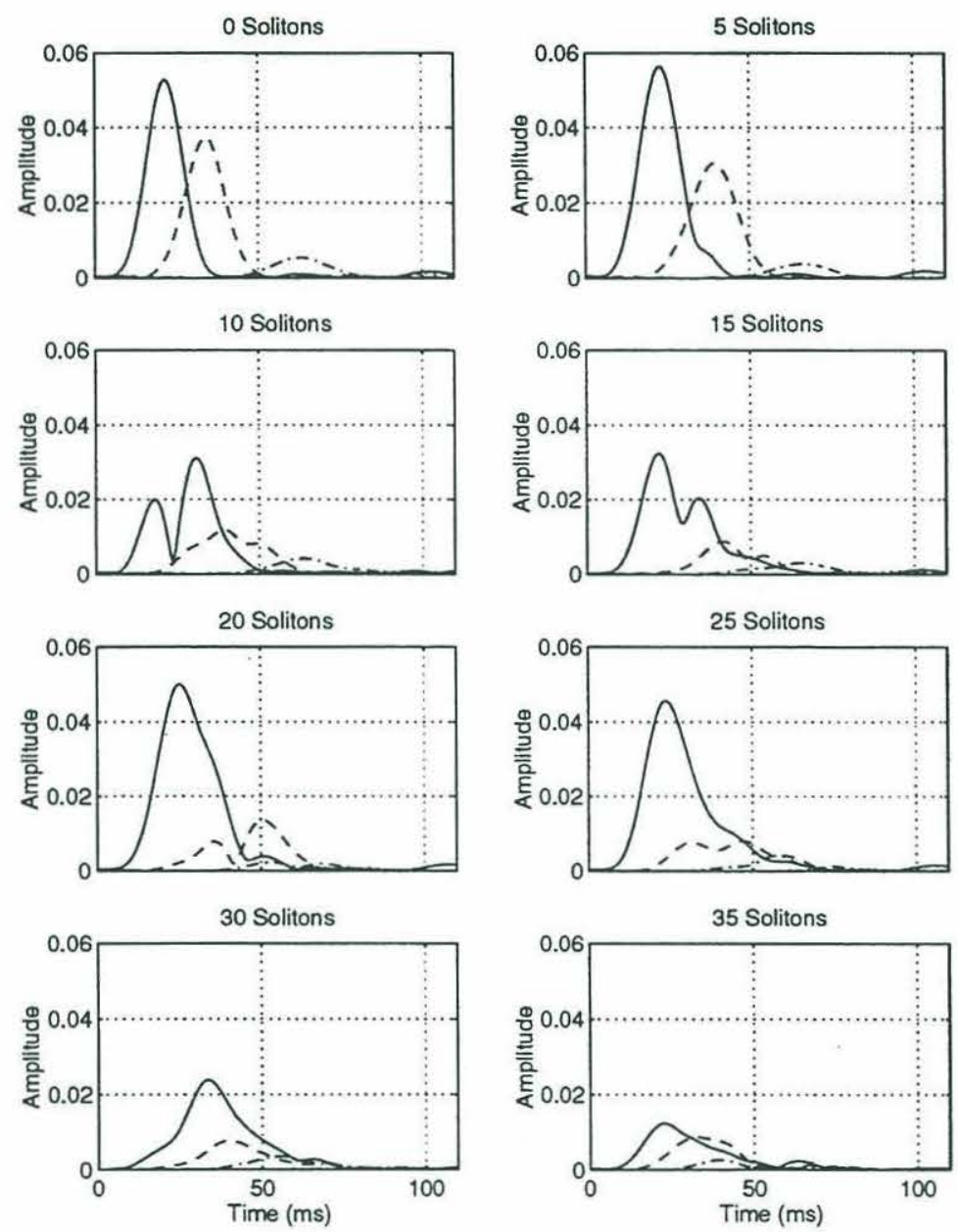

Figure 5-4: Single realizations of mode 1 (solid), mode 2 (dashed), mode 3 (dotdash), and mode 4 (dotted) arrivals for 0 to $35200 \mathrm{~m}$ SIA solitons over $32 \mathrm{~km}$. The simulated waveguides used are similar to those shown in Figure 5-3. The simulated source depth is $27 \mathrm{~m}$ in $68 \mathrm{~m}$ of water, and the compressional wave attenuation in the bottom is $.046 \mathrm{~dB} / \mathrm{m}$ at $400 \mathrm{~Hz}$. Note: Mode 4 arrivals are not visible at this level of attenuation. 

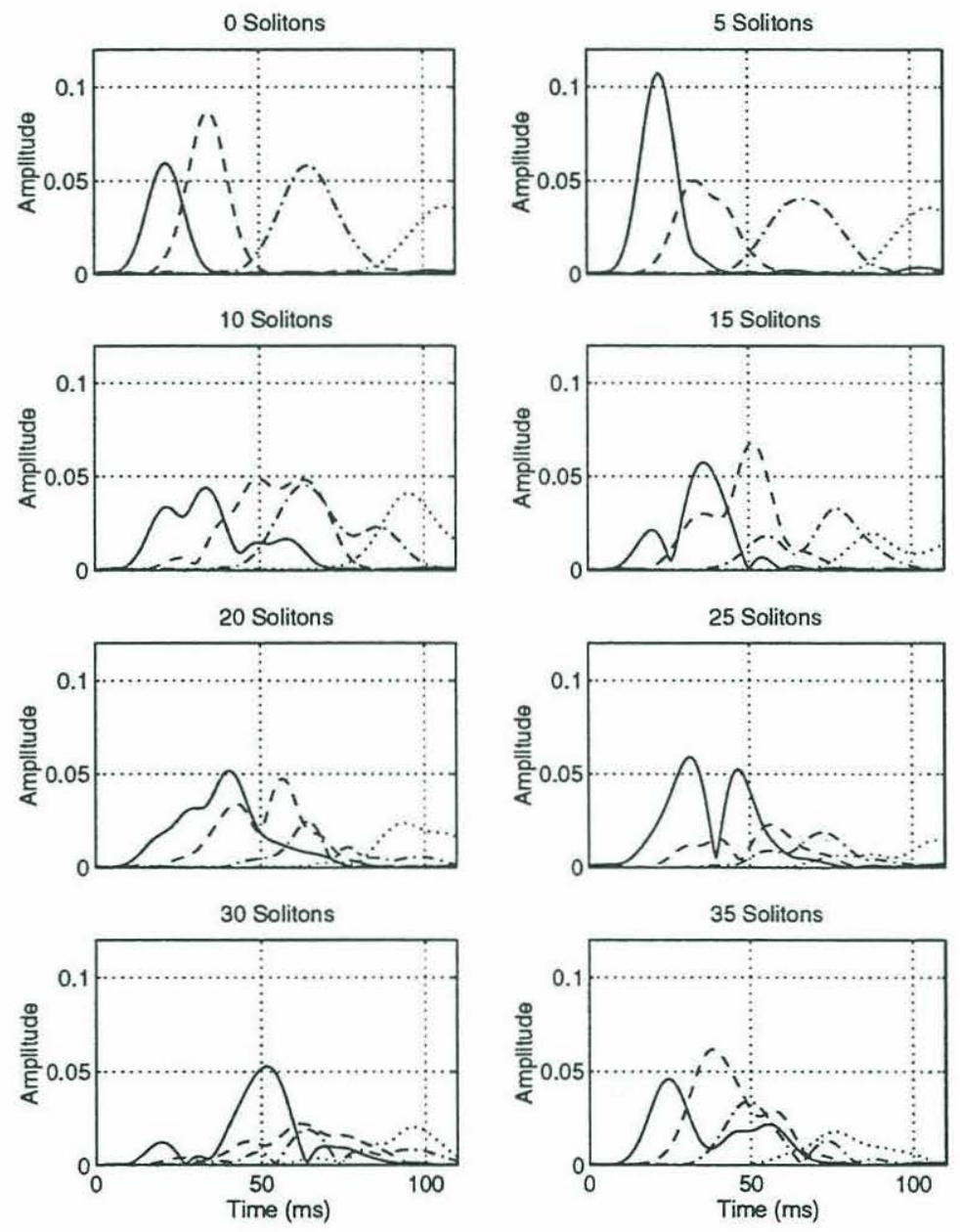

Figure 5-5: The same scenario as Figure 5-4, except the compressional wave attenuation in the bottom has been reduced from .046 to $0.0115 \mathrm{~dB} / \mathrm{m}$. 


\section{Peak of Mode 1}
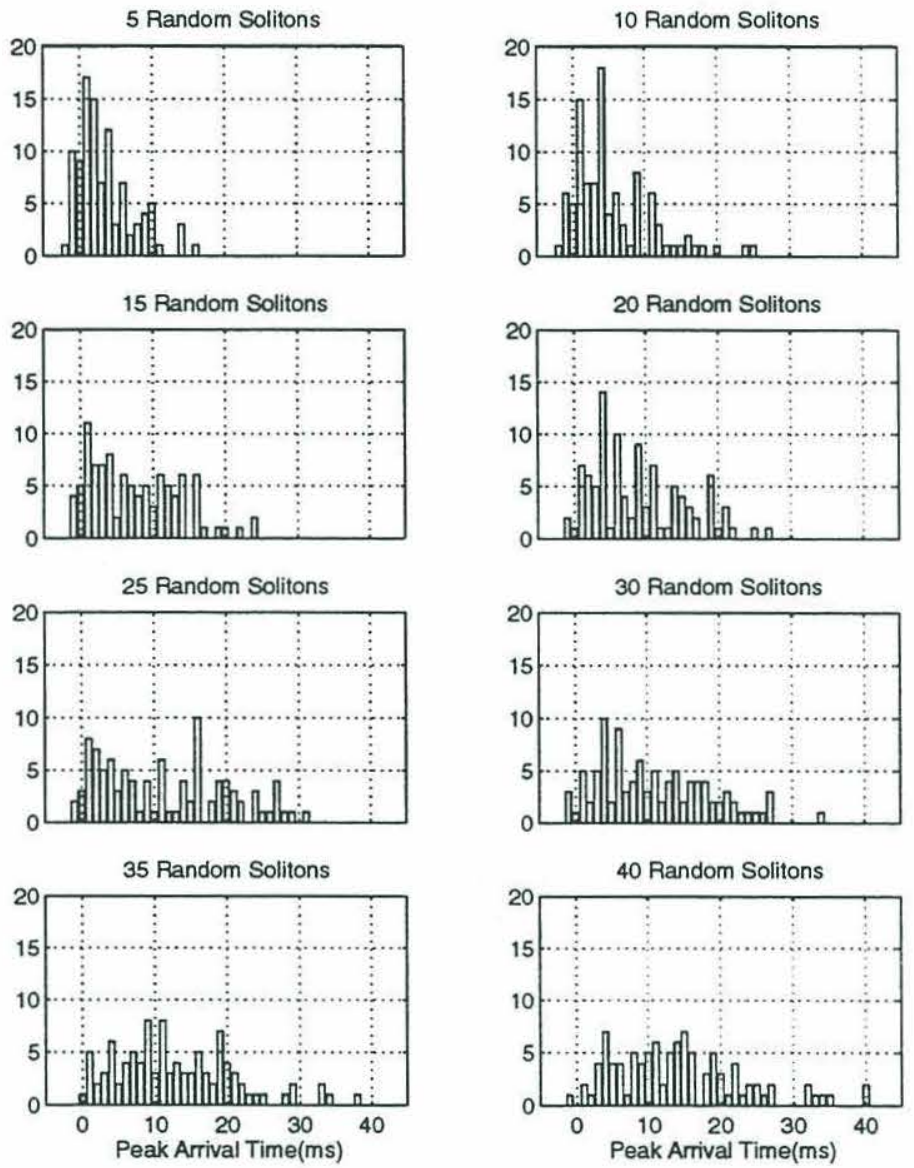

Figure 5-6: Histograms for peak position of mode 1 arrivals for 5 through 40 random solitons. The solitons start about $2.5 \mathrm{~km}$ from the source with an average separation of $450 \mathrm{~m}$ between soliton leading edges. Time zero corresponds to the PAM1 arrival.

\section{Soliton Number Effects}

Ten computer runs of 100 realizations each were conducted to evaluate the effects of adding a number of solitons between the source and receiver. The $200 \mathrm{~m}$ solitons are randomly placed with an average separation of $250 \mathrm{~m}$ between solitons, starting at an average distance of $2.5 \mathrm{~km}$ from the source. Figure 5-6 shows that the addition of solitons is clearly accompanied by an increase in the probability that dispersed mode 1 energy will dominate the arrival pattern. The averages of spread and bias statistics taken over the 100 trials are displayed as function of the number of solitons in Figure 


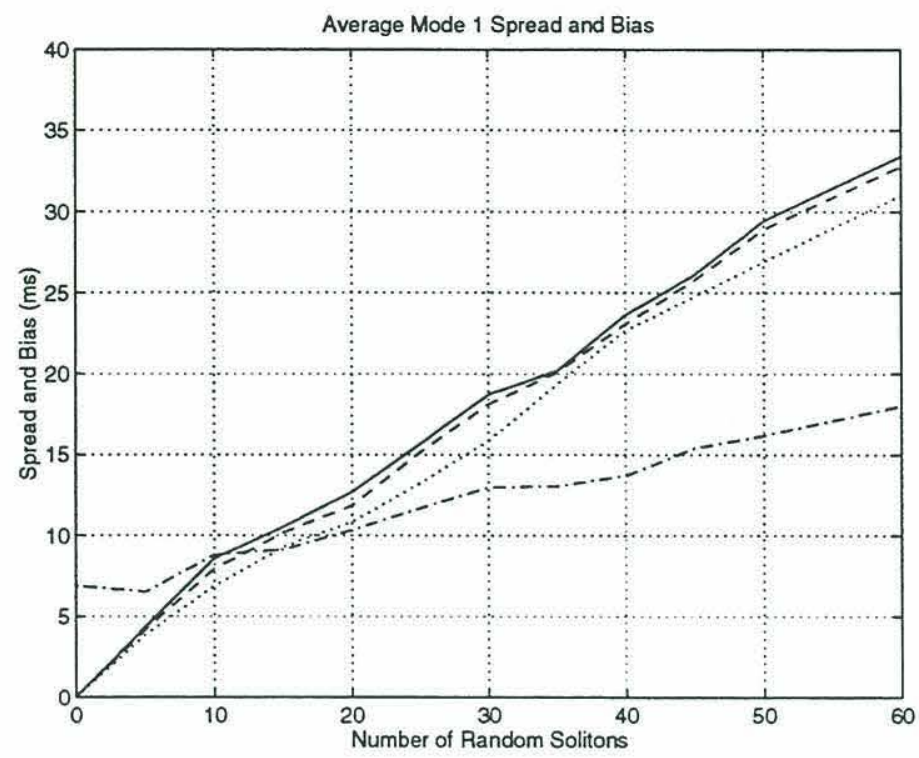

Figure 5-7: The average mean (-), median (- $)$, and peak $(\cdots)$ lag with respect to the pseudo-adiabatic arrival, and standard deviation (--) of scattered mode 1 arrivals plotted as a function of the number of solitons placed between the source and the receiver, where the solitons are added starting from the source end. The upper three lines are all bias measures, and the lower line is a measure of spread.

5-7. Note the close agreement between all three measures of signal bias; the small lags between the three are indicative of a fairly regular mean pulse shape with a longer tail on the trailing edge.

Figure 5-8 shows the situation to be entirely different if the solitons are added starting from the receiver end. It contains a re-plot of the data in Figure 5-7 plus additional curves for averages of spread and bias statistics when the solitons are added starting near the receiver.

Adding a few solitons near the receiver dramatically affects the spread of mode 1 arrivals, building quickly to a peak spread for about 20 solitons. The effect on bias is also dramatic when measured by the mean arrival, but it is less so when the median is used. Both of these measures of bias seem to be approaching asymptotic levels at the 25 soliton point. When peak position for less than 15 solitons is considered, no real difference can be seen between the "adding near the source" and the "adding near 


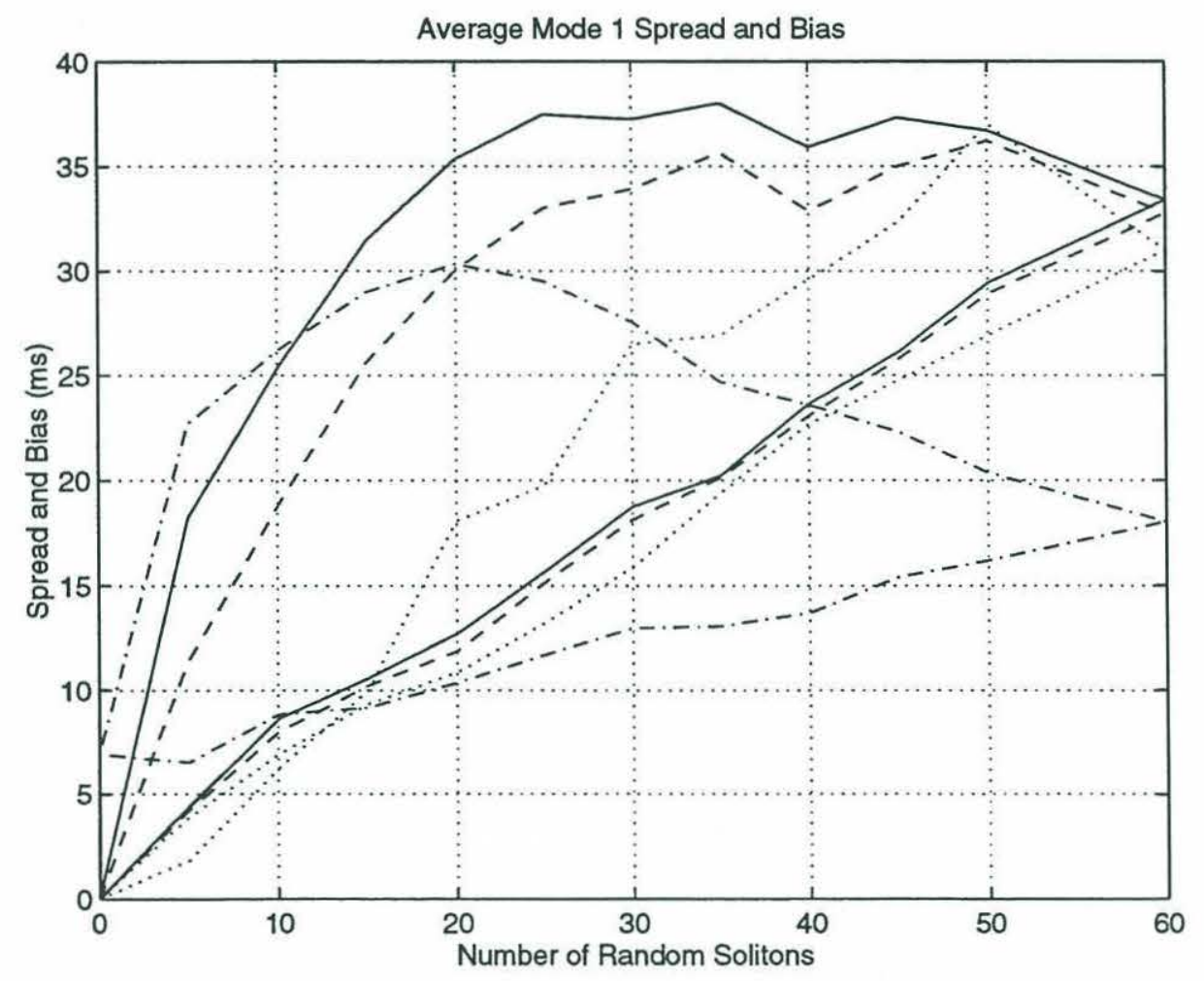

Figure 5-8: The average mean (-), median (- -), and peak ( $\cdots)$ lag with respect to the pseudo-adiabatic arrival, and standard deviation (-.) of scattered mode 1 arrivals plotted as a function of the number of solitons placed between the source and the receiver. The upper curves, averages over 50 realizations, represent the case where the solitons are added starting at the receiver end. The lower set of curves is replotted from figure 5-5. 60 solitons is essentially saturation, and the same points are used in both sets of curves. 
the receiver" results. The large differences between mean, median, and peak position for moderate numbers of near receiver solitons are indicative of a mean pulse shape with a substantial tail on the trailing edge.

All these effects can be understood, when one considers the amount of modal dispersion produced by this waveguide over a distance of $32 \mathrm{~km}$ (see Figure 4-9). If scattering interactions are constrained to occur only near the receiver, the peak of the mode 1 arrival is difficult to affect, but the tail of that arrival will be dramatically altered. The mean arrival time and the standard deviation of the arrival envelope are very sensitive to changes in the tail. The median and IQR of the arrival envelope are less sensitive, and the peak is by definition insensitive to the tail, unless, of course, the tail becomes the peak.

The asymptotic level of bias reached in the "adding near the receiver" case is to be expected. With no scattering, the history of the mode 1 arrival (single subarrival) is mode 1 only. As the number of scattering events is increased the mean modenumber history is weighted more and more by higher modes, but there is a limiting value for the average percentage of mode $\mathrm{n}$ history in a mode 1 arrival.

Dozier and Tappert [15] have shown in a simplified model of scattering with no modal attenuation that the equilibrium energy state is an equipartition of energy between the modes. The asymptotic limit for mode 1 modenumber history will, therefore, approach equal percentages, with some additional weighting (that depends upon the average distance required to randomly redistribute the energy in mode 1 , relative to the total distance traveled) given to mode 1. Similar arguments hold for the other modes. In more realistic scenarios, the asymptotic mode 1 modenumber history percentages will still approach the equilibrium energy partitions, but, as shown by Creamer [18], with attenuation these will not be equal percentages. The energy partitions and modenumber histories will be weighted towards the modes with the least attenuation.

The soliton effects on spread can be more easily visualized by considering the 
most simplistic case of two modes that sequentially scatter with all four coupling coefficients equal to 0.5 . For equal mode 1 and mode 2 intensities and equally spaced scattering events, with the last occurring just before the receiver, this scenario will yield an average mode 1 subarrival modenumber history of 50 percent travel in mode 1 and 50 percent travel in mode 2 . There will always be some variance between the individual subarrival histories with some subarrivals spending more time in mode 1 and others more time in mode 2, but the spread will decrease as the number of scattering events increases. For narrow pulses with adiabatic mode 1 and mode 2 travel-times separated by $\Delta T$, the spread of a mode 1 arrival, as measured by the $N$ normalized arrival standard deviation is

$$
\sigma=\frac{\Delta T}{2 \sqrt{n}},
$$

where $n$ is the number of scattering events and $N=2^{n}$ is the number of subarrivals. Of the $N$ mode 1 subarrivals, one is the PAM1 arrival, a second is coincident with PAM2 arrival (scattered off right before the receiver), and the remaining $2^{n}-2$ arrive in $k$-groups, where $k$ is number of increments traveled in mode 2 . The number of subarrivals in a $k$-group is given by the familiar formula,

$$
\frac{n !}{k !(n-k) !} \text {, }
$$

for finding the number of $k$-combinations of $n$ distinguishable items [19]. The PAM1 and "coincident with PAM2" arrivals can also be considered as $k$-groups, with $k$ equal to 0 and $n$, respectively.

Table 5.1 summarizes the subarrival statistics for the case where $n=4$ and $\Delta T=$ 1. Clearly, the same scenario with only one increment (i.e. one scattering at the very end) will still yield an average bias value of .50 , but the spread will be maximized (also equal to .50). Any additional scattering prior to this late event decreases the spread, with no change in bias. Conversely, the two-mode case with only a single 


\begin{tabular}{|c|c|c|c|c|c|c|}
\hline \multicolumn{7}{|c|}{$\begin{array}{l}\text { Simple Two Mode Scattering Scenario } \\
\text { with Four Evenly Spaced Increments }\end{array}$} \\
\hline & \multicolumn{5}{|c|}{ Subarrival $k$-group } & \multirow[b]{2}{*}{ Total } \\
\hline & 0 & 1 & 2 & 3 & 4 & \\
\hline Number in Group & 1 & 4 & 6 & 4 & 1 & 16 \\
\hline Group Bias & 0 & .25 & .50 & .75 & 1.00 & .50 \\
\hline Group Spread & 0 & 0 & 0 & 0 & 0 & .25 \\
\hline
\end{tabular}

Table 5.1: Subarrival statistics for the simple two mode 50/50 scattering scenario where there are four equally spaced increments and the difference between the PAM1 and PAM2 arrival times is $\Delta T=1$.

scatter occurring early in the path will produce a small amount of spread and bias; additional scattering events occurring later will cause an increases in both.

The Figure 5-8 simulation runs are more complicated than the above two-mode example, but the same effects can be seen. The eight-mode cross-modal coupling coefficients are much less than 0.5 , so the "adding near the receiver" peak spread is not reached until around 20 solitons. We can also see the bias stabilize at about the same number of solitons, indicating saturation in mean modenumber history of subarrivals. The two-mode "adding near the receiver" example was saturated from the outset.

\section{Soliton Location Effects}

The above section has illustrated, via Figure 5-8 in particular, that the general location of solitons in a waveguide has more impact on spread and bias levels than does the number of solitons. In this section we will show that, in addition to the general location, the specific location of solitons contributes significantly to the nature of mode 1 arrival patterns. Figure 5-9 demonstrates this with two trains of six SIA solitons propagating in lock-step down a waveguide at $0.8 \mathrm{~m} / \mathrm{s}$. As the soliton trains progress towards the source, the location of the last scattering event moves towards the source, reducing the potential for mode 1 arrival bias. As expected, this 
general trend in mean and peak arrival bias is evident in Figure 5-9, but, even more interesting is the fact that the mean and peak arrival time bias is very sensitive to small shifts in soliton train positions.

Figure 5-10, a companion to Figure 5-9, provides a good qualitative sense of the mode 1 arrival pattern's dependence on the position of the soliton trains. The outline of the first arrival at the bottom of the figure shows four distinct humps that roughly correspond in location to the mode 1-4 arrivals shown in Figure 4-9. For the majority of the arrivals (see Figure 5-9), the peak arrival resides on the mode 1 hump, but it often, almost periodically, shifts to the mode 3 hump, and it occasionally resides on the mode 2 hump. The mean arrival fluctuations are equally dramatic. The sensitivity of the arrival shapes and bias measurements to small changes in the location of solitons is chiefly due to the constructive and destructive interference patterns of scattered subarrivals. The undulations evident in the "mode 1" hump on Figure 5-10 contribute significantly to the peak position jumps in Figure 5-9. The source of these undulations is alternating constructive and destructive interference between mode 1 and 2 in the first soliton train. The mode cycle distance between mode 1 and 2 is about 1420 $\mathrm{m}$; this combined with a train speed of $48 \mathrm{~m}$ per minute, yields the half hour cycles evident in both figures.

Figure 5-11, a companion to Figure 5-10, shows two snapshots of the arrival shapes for all eight modes propagated in this simulation. The left hand column shows the minute 0 arrival shapes, and the right column shows the minute 300 arrival shapes. A comparison of the two columns shows the movement of the two soliton trains towards the source markedly reduces the "spread level" for all eight modes. The thinner lines in this figure represent predicted arrival shapes for modes that propagate adiabatically (i.e. no scattering of energy between modes). The eighth mode is only minimally excited by the source at $27 \mathrm{~m}$ depth, and it undergoes the most attenuation, so it is not even visible as an unscattered arrival. There is little modal dispersion beyond mode 6 , and as a result, the scattered arrivals for modes 6-8 show only negative spread 

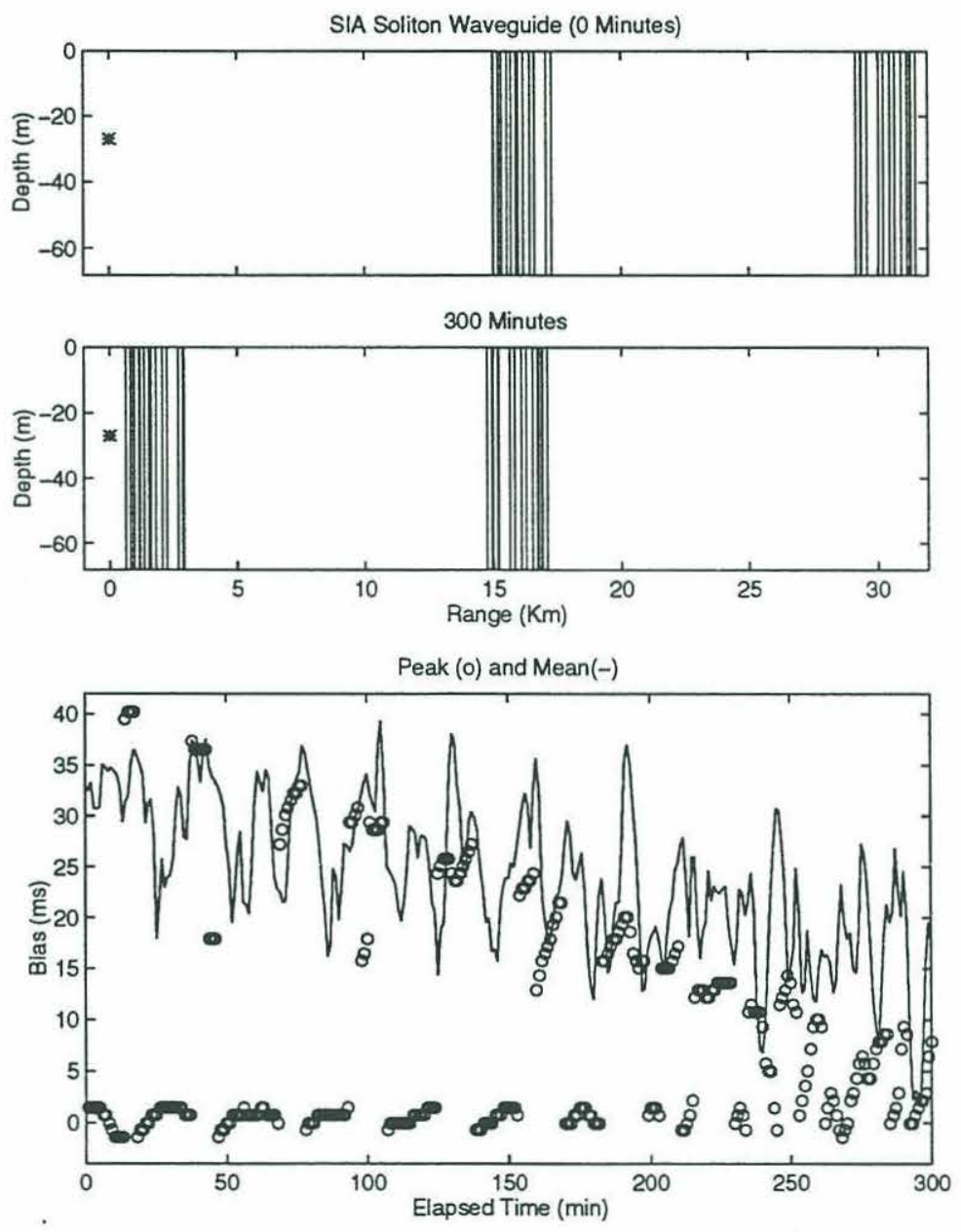

Figure 5-9: Time dependent waveguide with two sets of six SIA solitons. The upper frame shows the starting positions, the middle frame shows the positions five hours later, and the lower frame is plot of peak position bias (re PAM1) as a function of time. The solitons propagate in lock-step, traversing $48 \mathrm{~m}$ per minute, the time between each peak position data point (o). The mean arrival data is also in one minute increments, but the points are connected by a solid line. 


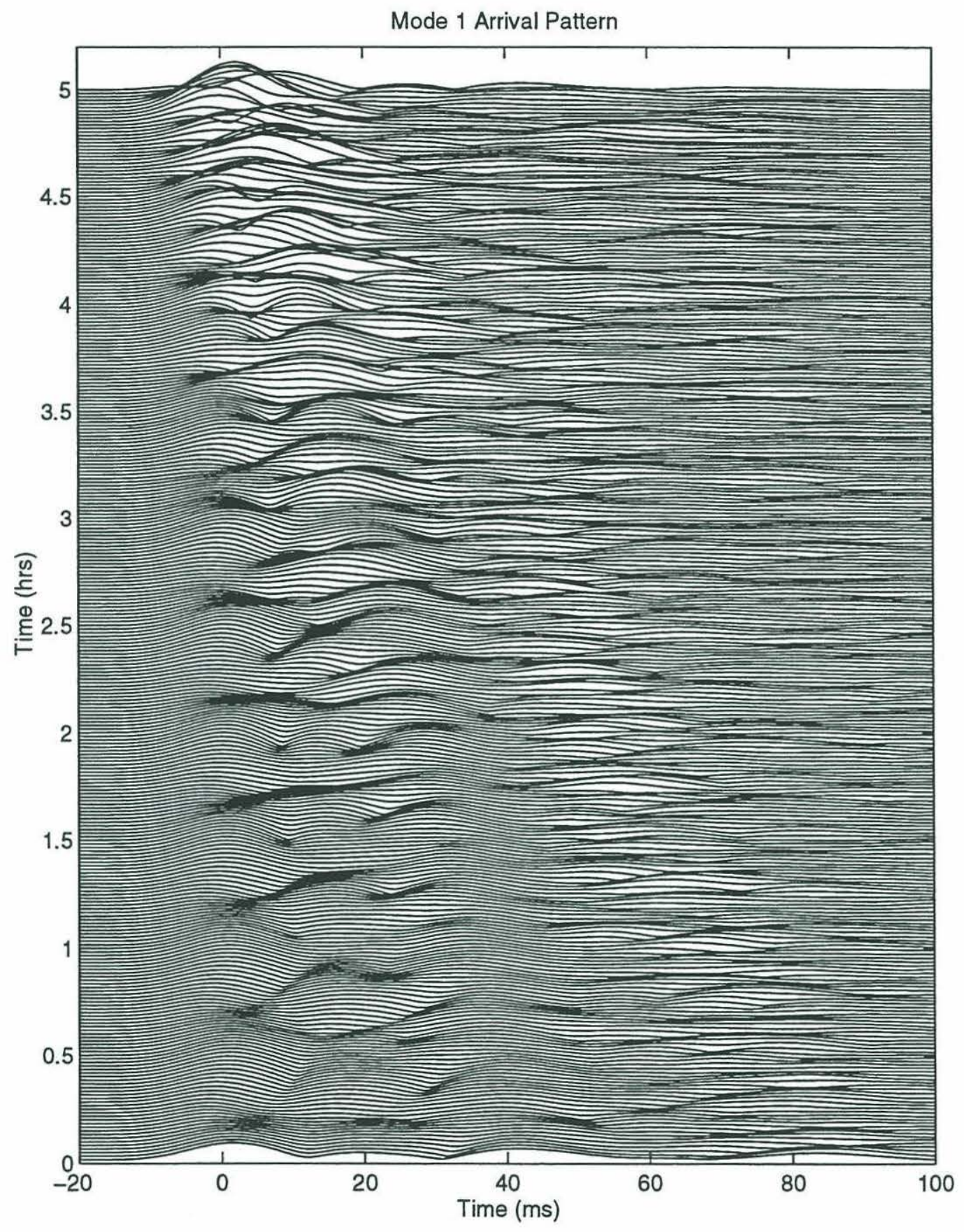

Figure 5-10: Waterfall of mode 1 arrival patterns from the time dependent waveguide with two sets of six SIA solitons. The 300 peak positions in the lower frame of Figure 5-9 were picked from these arrival patterns. 

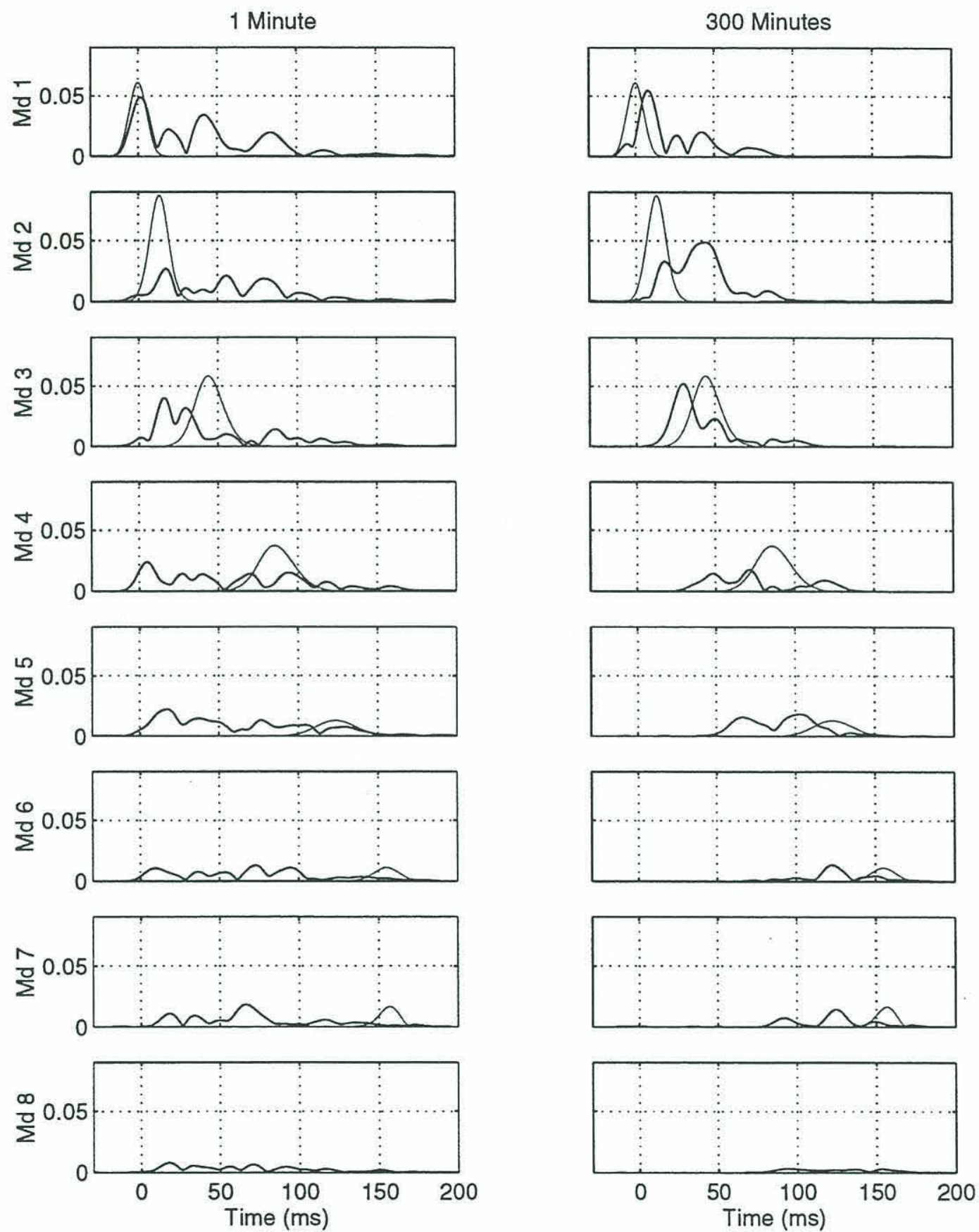

Figure 5-11: Snapshots of the arrival shapes for all eight modes propagated in the Figure 5-10 simulation. The left hand column shows the minute 1 arrival shapes and the right column shows the minute 300 arrival shapes. The thicker lines are the scattered arrival shapes, and the thinner lines show the corresponding unscattered mode arrivals. The eighth mode is only minimally excited by the source at $27 \mathrm{~m}$ depth, and it undergoes the most attenuation, so it is not even visible as an unscattered arrival. 


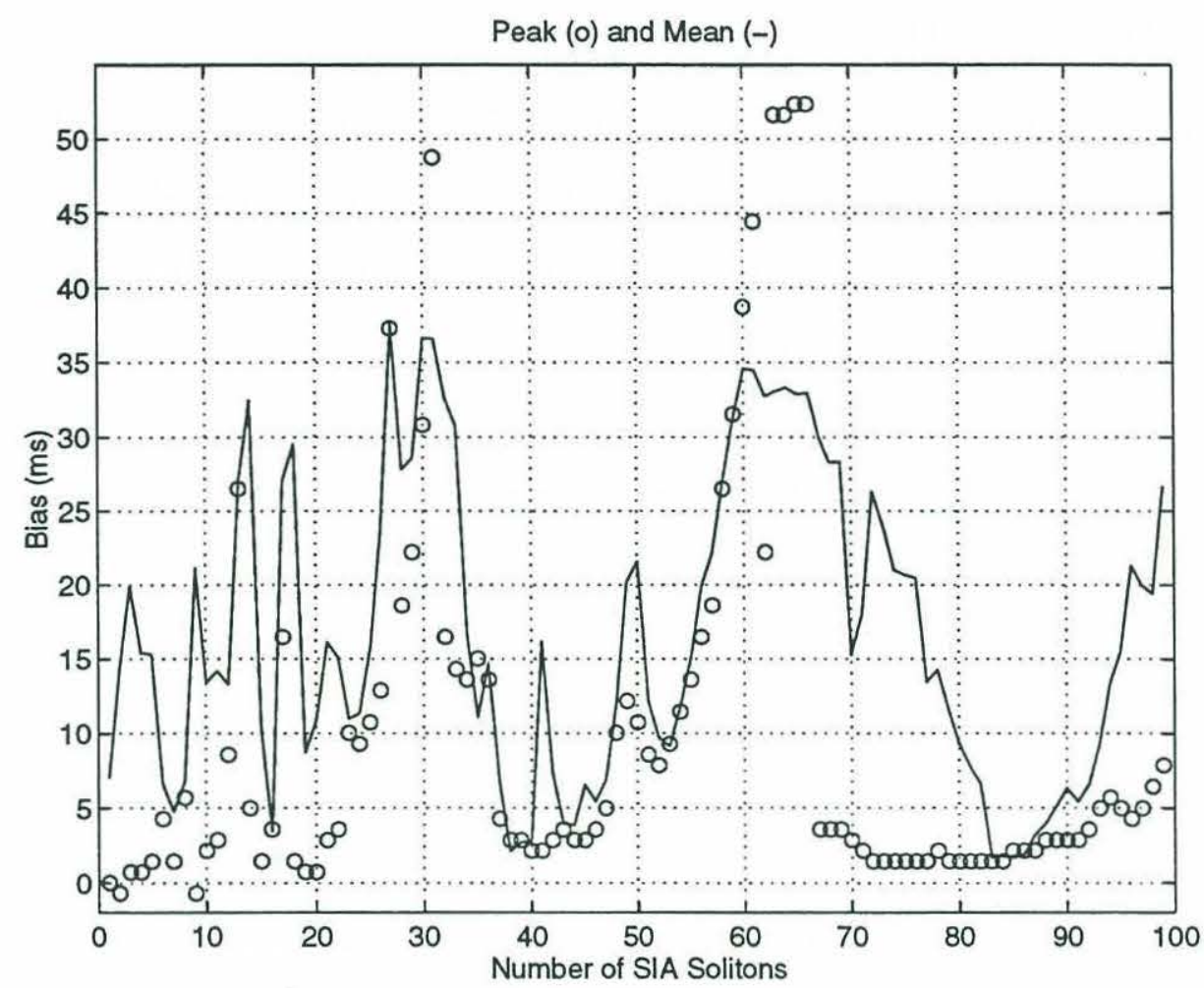

Figure 5-12: A comparison of mean (-) and peak (o) arrival time as function of the number of evenly spaced solitons in a $32 \mathrm{~km}$ waveguide. The first soliton is always $200 \mathrm{~m}$ from the source and the last soliton is roughly $32 \mathrm{~km}$ divided by the number of solitons away from the receiver.

in travel time.

Another example of the sensitivity of mode 1 bias to specific soliton location can be observed by considering mode 1 bias level as a function of the number of evenly spaced $200 \mathrm{~m}$ SIA solitons in a $32 \mathrm{~km}$ waveguide. The first soliton is set at $200 \mathrm{~m}$ from the source, the rest are evenly spaced between there and the receiver. An additional soliton in the arrangement creates a series of small changes in soliton location and spacing; their cascading effects cause the large changes in the mode 1 arrival bias plotted in Figure 5-12. 


\section{Source Excitation Effects}

We will now look at one final contributor to mode arrival shape fluctuations. In a conceptual sense, the mode excitation at the source determines the size of the individual mode pulses leaving the receiver; these pulse sizes in turn control the size of all the descendent subarrivals, which will in turn combine to form the mode arrivals at the receiver.

Solitons routinely cause upwards of $10 \mathrm{~m}$ depressions in the thermocline; the effects of these depressions can be seen in the upper frame of Figure 5-13 which shows a 2 hr record from the shallowest thermistor on the WVLA. The source excitation levels (source depth of $29 \mathrm{~m}$ ) in the middle frame were obtained from a series of mode shapes derived from the same 2 hrs of WVLA thermistor data. A depth of $68 \mathrm{~m}$ was used for the mode shape calculations. The waveguide for this simulation is similar to the ones shown in Figure 5-9, except it is frozen (i.e it does not move); the source excitation is the only parameter that changes. The leading edge of the first train was fixed at $5 \mathrm{~km}$ from the source and the trailing edge in the second train was fixed at $5 \mathrm{~km}$ from the receiver. The resulting shifts in the mean arrival are quite dramatic, but the mode excitation changes are insufficient to shift the peak significantly.

\subsection{Analysis of the "Propagated Thermistor String Model"}

\subsubsection{An Example Waveguide}

The fine scale "propagated thermistor string" model described in Chapter 4 is much slower than the SIA model, but the use of thermistor string data brings more realistic size and spacing characteristics for solitons, as well as added scattering from other sources (e.g. linear internal waves). An example of this type of waveguide overlaid with isopycnal surface displacements taken from a high frequency backscatter record 

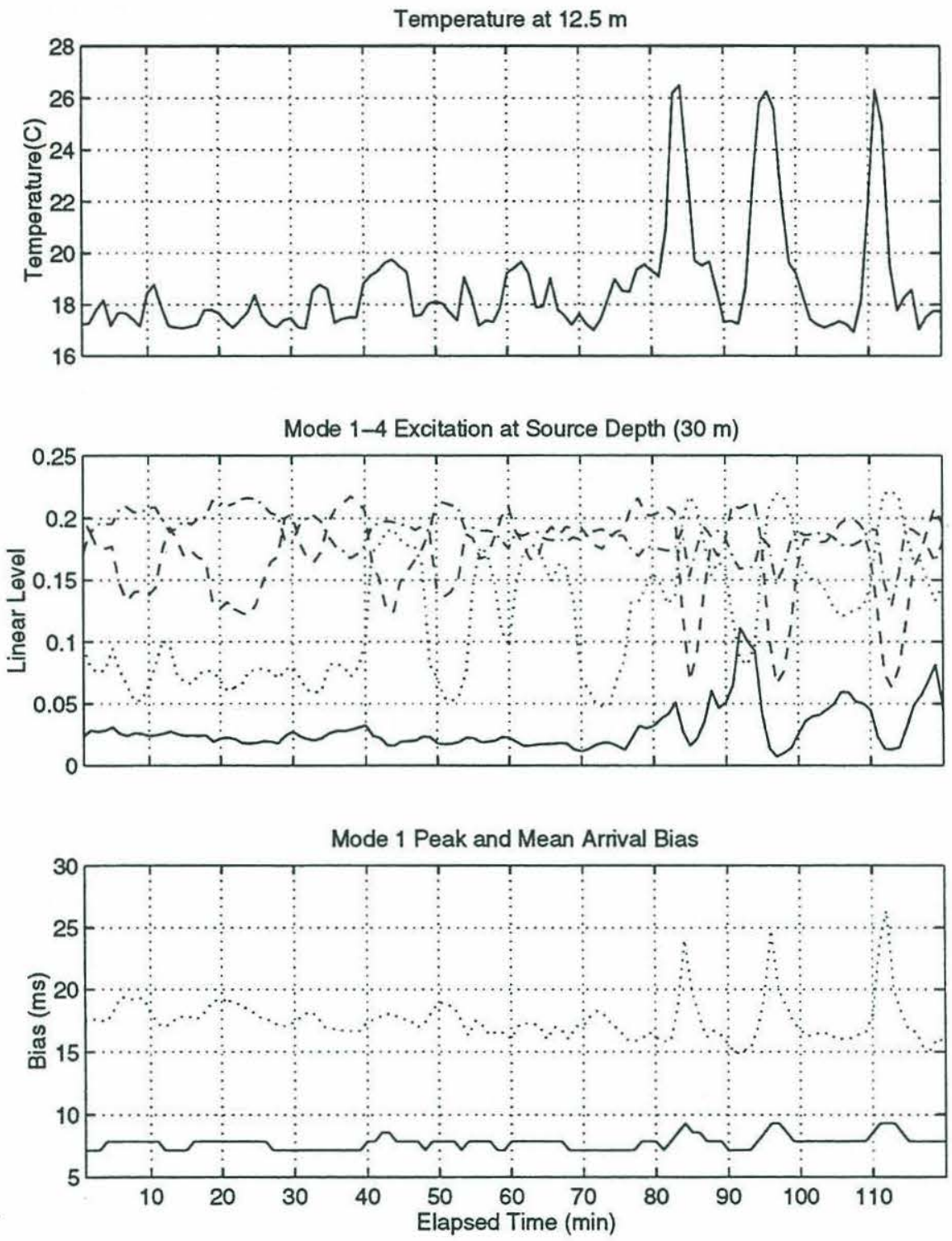

Figure 5-13: The top graph shows $12.5 \mathrm{~m}$ depth temperature readings from the $2 \mathrm{hrs}$ of thermistor data used to simulate modal excitation levels. The middle graph shows the resulting modal excitation levels for mode 1 (solid), mode 2 (dashed), mode 3 (dot-dash), and mode 4 (dotted). Mode 5 is similar in magnitude and used as an input to the model, but it is omitted from this figure to avoid clutter. The lower graph shows the output bias levels corresponding to the excitation inputs shown in the middle graph. The solid line is the mode 1 arrival envelope peak arrival time in excess of the PAM1 arrival time and the dotted line is the mode 1 arrival envelope mean arrival time in excess of the PAM1 arrival time. 
provided by Marshall Orr (NRL, Washington D.C., private communication) is shown in Figure 5-14. The waveguide in this case is produced from records from a dedicated thermistor string (not the WVLA thermistors) used during SWARM. This "T-string 598" data was used in this comparison with the Orr backscatter record, because the WVLA was not in the water early enough to record these events. Various comparisons between different instruments point to non-linear internal wave propagation speeds in the range of 0.6 to $0.8 \mathrm{~m} / \mathrm{s}$. The propagation speed of $0.62 \mathrm{~m} / \mathrm{s}$ used in Figure 5-14 was chosen for best overall agreement between the records. The agreement is, in fact, quite good - better than one would have expected using the "constant velocity" assumption for soliton and internal tide propagation.

\subsubsection{Model Results}

The WVLA, with its thermistors spanning $12.5 \mathrm{~m}$ to $60.5 \mathrm{~m}$ in depth, provides better waveguides for simulating tomographic transmissions. The sound speeds are extrapolated for depths above $12.5 \mathrm{~m}$ and below $60.5 \mathrm{~m}$, and by assuming a WVLA temperature signal propagation speed of $.8 \mathrm{~m} / \mathrm{s}$ along the acoustic path, each 670 minutes of thermistor string data is transformed into a 32160 by $68 \mathrm{~m}$ waveguide. The profile at the source determines the "eight mode" starting field for the model, and an "eight mode" transmission is then propagated through the waveguide and relevant statistics are obtained from the mode arrivals.

\section{Peak Arrivals}

Figure 5-15 shows several days of mode 1 peak arrival times in both absolute and wander-corrected terms. There are six minutes between each acoustic transmission, and, in order to provide some qualitative sense of the variability in the strength of the arriving signals, the size of each arrival dot is scaled by the amplitude of the peak arrival. The peak arrivals consistently display a tendency towards positive travel time bias relative to the PAM1 arrival, but there is a notable exception for the period 


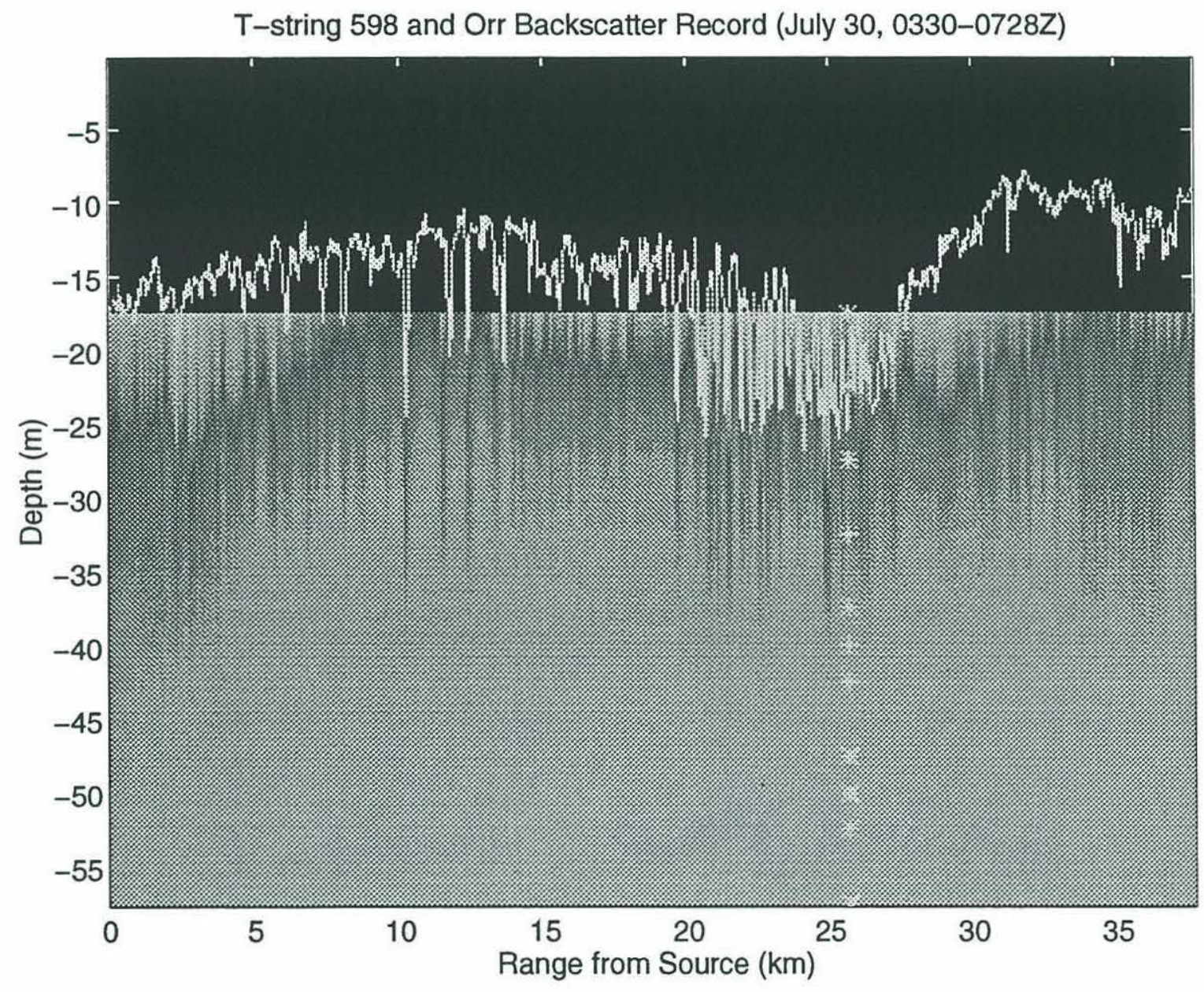

Figure 5-14: Pycnocline displacements from an Orr backscatter record compared to a thermistor string record using a propagation speed of $.62 \mathrm{~m} / \mathrm{s}$ over the distance between the back scatter instrument and the thermistor string at each backscatter reading. 
"Propagated Thermistor String" Model
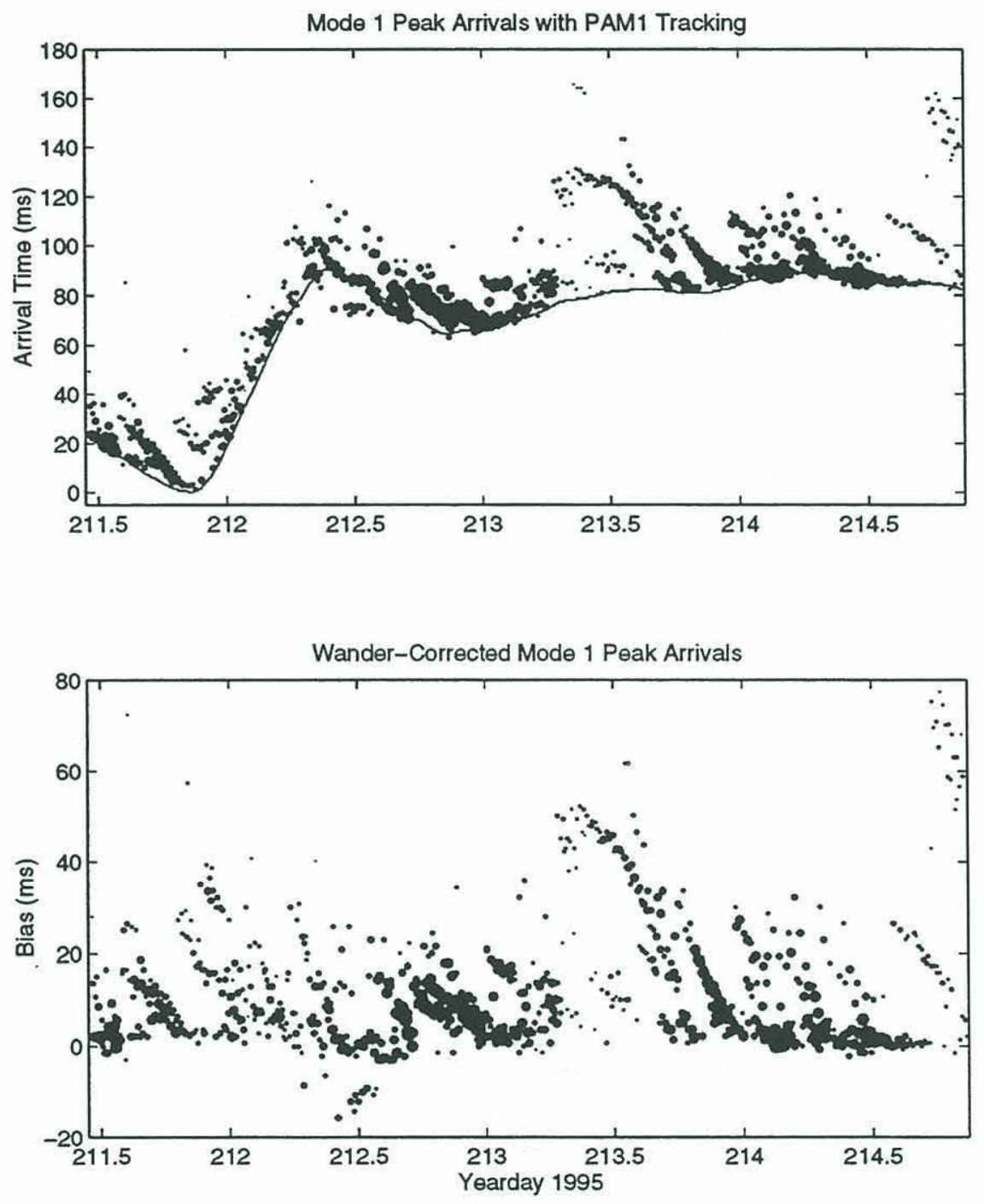

Figure 5-15: Distribution of peaks for the mode 1 arrivals of simulated transmissions using sequential WVLA thermistor string generated waveguides. The sequential 32160 by $68 \mathrm{~m}$ waveguides are separated by six minutes. The size of each arrival dot is scaled by the amplitude of the peak arrival. The upper frame includes a plot of the PAM1 arrival time in conjunction with the individual peak arrival times; the zero reference on the arrival time axis corresponds to 21.6 seconds. The lower frame plots the bias or offset of the peak arrivals relative to the PAM1 arrival time for each realization. 
around day 212.5. During this period, the PAM2 arrival is actually earlier than the PAM1 arrival, allowing for negative bias in some mode 1 arrival times.

For periods where the PAM1 arrival is the earliest arrival, the distributions of mode 1 peak arrivals tend to exhibit a fairly clean leading edge that coincides with the PAM1 arrival time. The arrivals with the largest peak amplitudes tend to fall within 10 to $20 \mathrm{~ms}$ of this leading edge. Arrivals with peak bias levels in the 40 to $60 \mathrm{~ms}$ range are partially the result of destructive interference among the early subarrivals, and as a result, the peak heights are noticeably smaller.

The story for mode 2 arrivals is somewhat different. Figure 5-16 displays the peak mode 2 arrival distributions obtained from the simulated transmissions. The temporal variations in peak amplitude are much stronger for mode 2 , while the correlation between bias level and peak amplitude appears weaker relative to the mode 1 relationship. Also, save for the period where the PAM2 arrival is earlier than the PAM1 arrival, we generally see significant peak arrival time bias in both the negative and positive directions, so, unlike the PAM1 arrival time, the PAM2 arrival time is not readily discernible from the total distribution of mode 2 peak arrivals. The PAM1 arrival was generally discernible, because it was usually "smeared" in only one direction.

A simple "thought experiment" may prove useful. Consider a curve thickly drawn in wet paint across a clean white surface. Now from one side of the curve, direct a high velocity wind across its surface. The paint will smear, but only in one direction; the original shape of the curve is still discernible. However, if the winds are instead directed in a random fashion from both sides of the curve, the original shape will be lost.

Therein lies the problem with the mode 2 and higher arrivals. They are "smeared" in both directions, severely limiting their utility in standard tomographic inversions for range-average soundspeed profiles. However, the smearing levels themselves may prove to be a useful input to an inversion for something else in the future (e.g. location 


\section{"Propagated Thermistor String" Model}
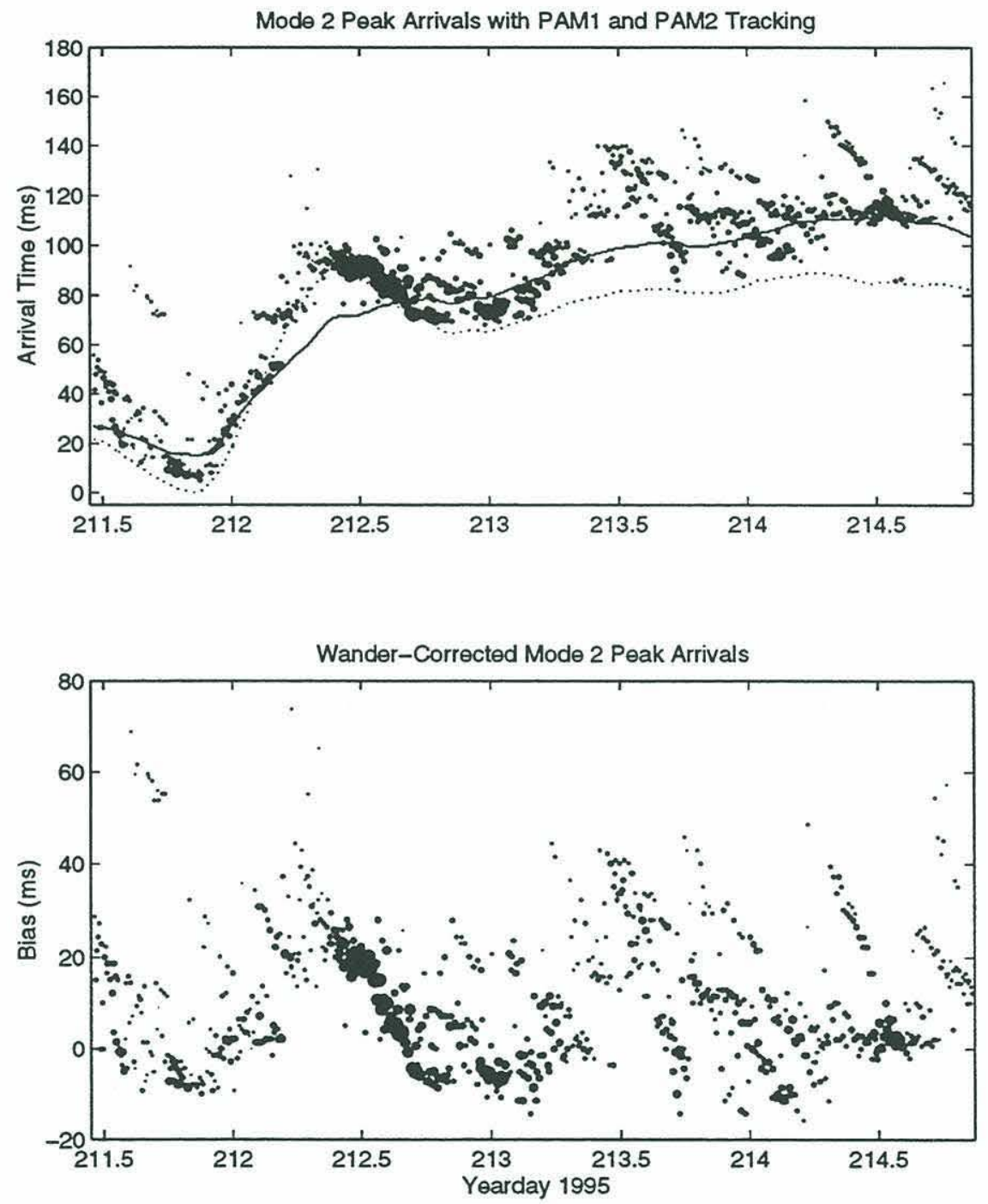

Figure 5-16: Distribution of peaks for the mode 2 arrivals corresponding to the mode 1 arrivals of Figure 5-15. The upper frame includes a plot of both the PAM1 arrival time (dotted line) and PAM2 arrival time (solid line), in conjunction with the individual peak arrival times; the zero reference on the arrival time axis corresponds to 21.6 seconds. The lower frame plots the bias or offset of the mode 2 peak arrivals relative to the PAM2 arrival time for each realization. 
of a soliton train).

\section{Spread Statistics}

The interquartile range (IQR) of a mode arrival envelope provides a robust estimate for that mode's time spreading. Figure 5-17 plots these spread statistics for mode 1 and 2 , in 30 minute bin averages, as an overlay with the WVLA temperature profiles for the simulation test period. For a given spread reading the coincident temperature profile was used in modeling the SSP at the receiver. The profile at a time 0.46 days to the left was used at the source, and the profiles in between were used in the interior of the modeled $32,000 \mathrm{~m}$ waveguide.

On average, larger amounts of spread tend to be accompanied by a significant amount of soliton activity in the latter half of the waveguide (i.e. in the region from the time in question to about 0.25 days to left), but this is not always the case. The large peaks in the the mode 1 spread history around yeardays 213.4 and 214.8 , for example, are almost inexplicable in terms of the accompanying soliton activity in the waveguide or the excitation levels at the source. These are further examples of the sensitivity of spread and bias statistics to the details of the modeled waveguide. Mode 2 spread, on the other hand, seems to be more closely tethered to the "general level" of soliton activity, showing a near tidal periodicity in spread fluctuations.

One other notable region of divergence between the two spread histories is in the region around day 212.5. Mode 1 shows moderate spread, while the spread of mode 2 is at near minimum, and the corresponding soliton activity is low to moderate. Fluctuations in soliton activity are probably not the main source of spread fluctuations during this period, rather Figure 5-15 reveals this to be the region where the PAM2 arrival time is shifting significantly relative to the PAM1 arrival time.

The correlation between spread levels and soliton activity is difficult to quantify; there is no single statistical measure of soliton activity. If the standard deviation of the temperature at $22.5 \mathrm{~m}$ depth over the previous $4.3 \mathrm{hrs}$ is used as a crude measure 

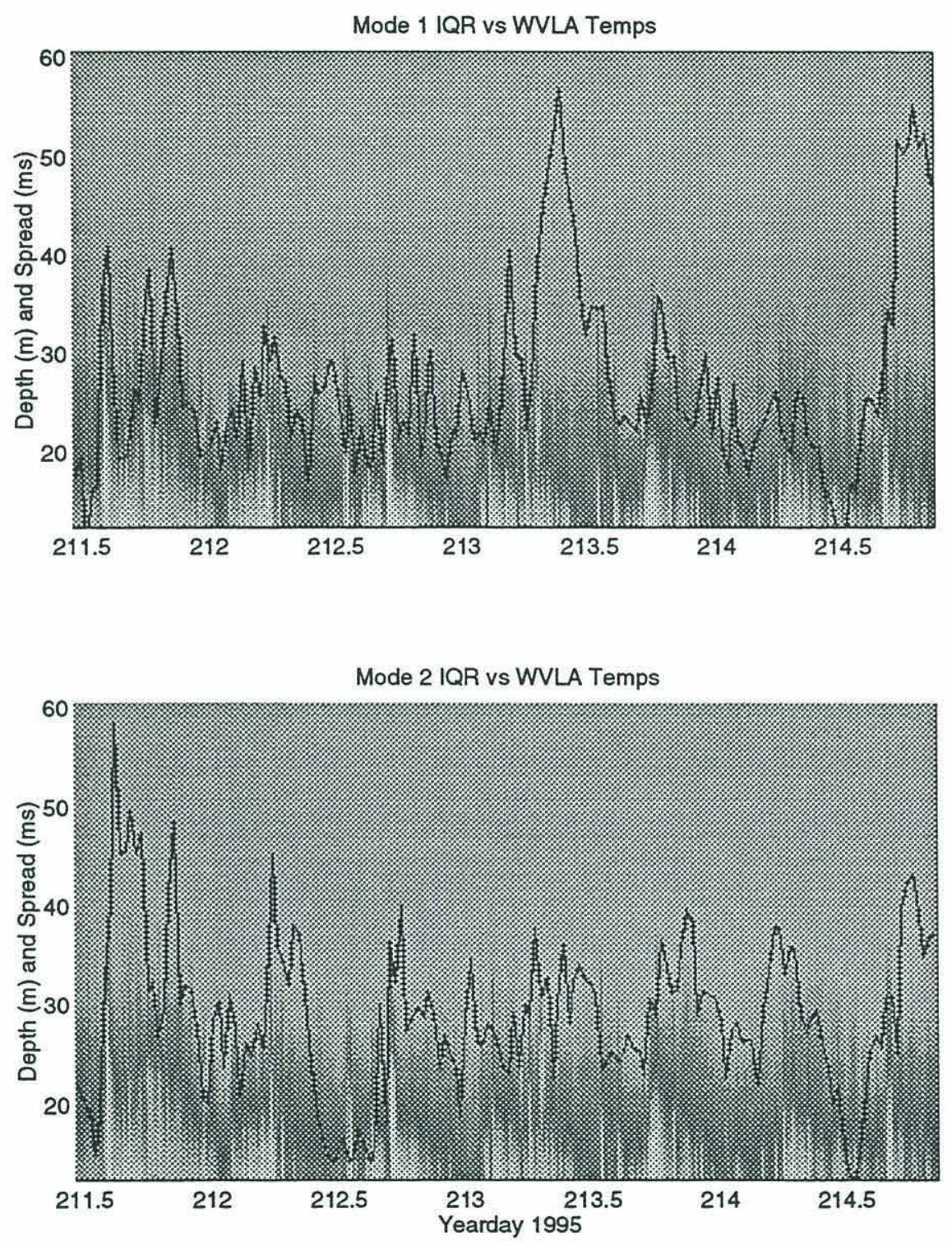

Figure 5-17: 30 minute bin averaged IQR measurements and the WVLA temperature profile for the simulation test period. The profiled temperatures range from 7.2 to 25.9 degrees $\mathrm{C}$. The top frame is for mode 1 and the bottom is for mode2. 
of soliton activity near the receiver, then an attempt at measuring the correlation can be made. Unlike spread levels, this statistic cannot change appreciably from minute to minute, but the long $(\sim 12 \mathrm{~km})$ reach is necessary to encompass the most critical of region soliton scattering. Figure 5-18 shows $1 \mathrm{hr}$ bin averaged spread statistics plotted against $1 \mathrm{hr}$ bin averages of this statistic for modes 2-4. Here we see similar rise and fall patterns in all the statistics, and computed zero-lag cross-correlations between the temperature statistic and the mode $2-4$ spread levels are $.43, .14$, and .17 respectively (all relative peaks in correlation, except mode 4 which peaks at .34 with a $3 \mathrm{hr}$ lag). Similarly, the zero-lag cross-correlation between the temperature statistic and mode 1 spread is .34 .

The search for a better general soliton activity statistic, in terms of correlation with spread levels, will be difficult; there are several contributing factors to consider. Small changes in the position and spacing of solitons, as well as variations in mode excitation at the source, can have a significant impact on spread statistics, and almost any general measure of soliton activity imaginable will only change appreciably over hourly or greater time scales. Spread levels in both simulated and actual data can change appreciably in less than one minute.

As observed in Figure 5-15, increased bias in mode 1 peak position is generally accompanied by a reduction in the mode 1 peak height. The same relation holds between spread and peak height, and the correlation is fairly strong, as evidenced by Figure 5-19. This figure also shows that peak height fluctuations for mode 2 have a negative a zero-lag correlation with mode 1 spread statistics, presumably the result of a corresponding "smearing" of mode 2 energy. However, this negative correlation is diminished as the mode number increases. This sort of analysis can be performed using the spread statistics from higher modes; a tabulations of the zero-lag results are provided in Table 5.2. Not unexpectedly, the amounts of correlation vary considerably between modes, reflecting the complicated relationship between the degree of modal coupling and the amount of spread and peak intensity in higher mode number arrivals. 
Spread (ms) and T-String STD (centidegrees)
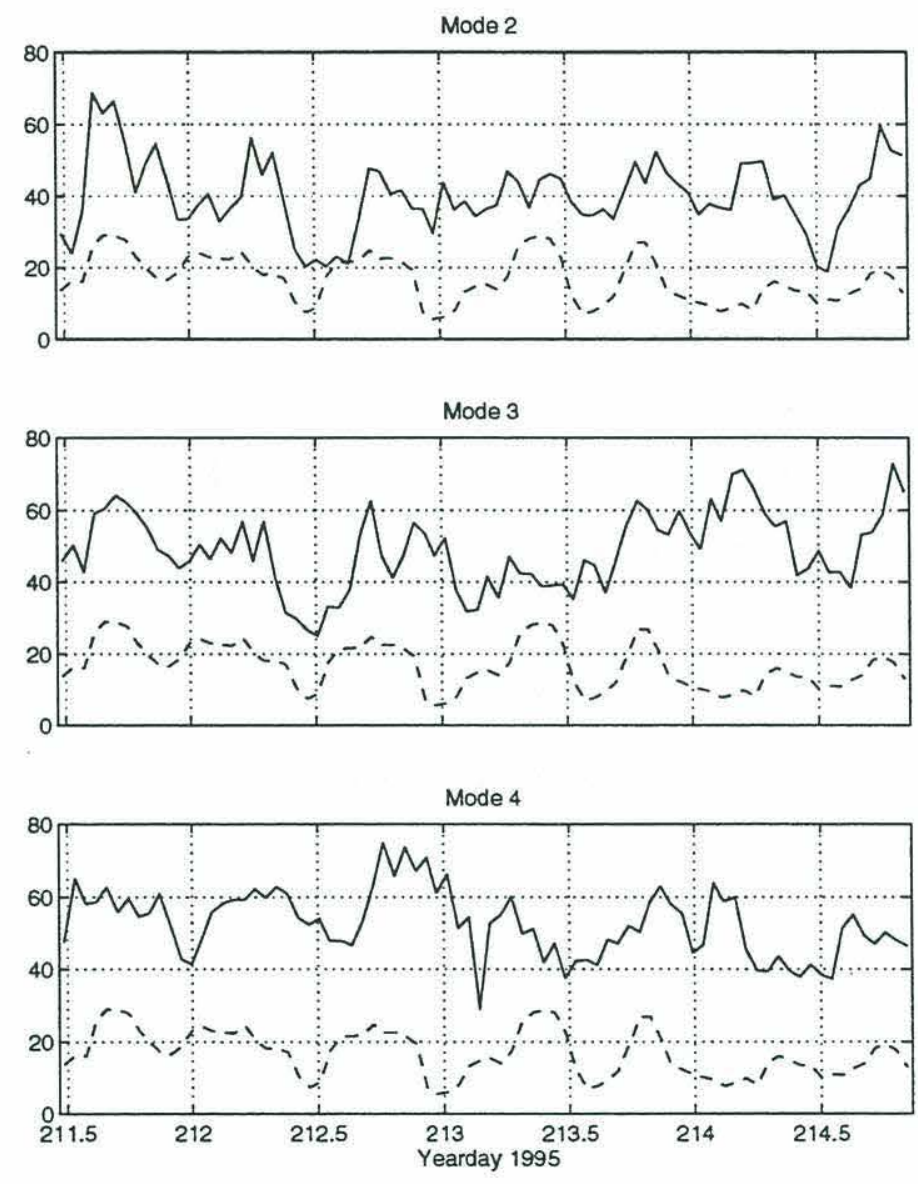

Figure 5-18: A comparison of 1 hour bin averaged IQR levels for the simulated mode 2-4 data (solid lines) and a $1 \mathrm{hr}$ bin average of the Standard Deviation of the Temperature at $22.5 \mathrm{~m}$ depth over the previous $4.3 \mathrm{hrs}$ (dashed lines). The standard deviation of the temperature is in tenths of a degree centigrade to facilitate comparison with the IQR levels in milliseconds. 


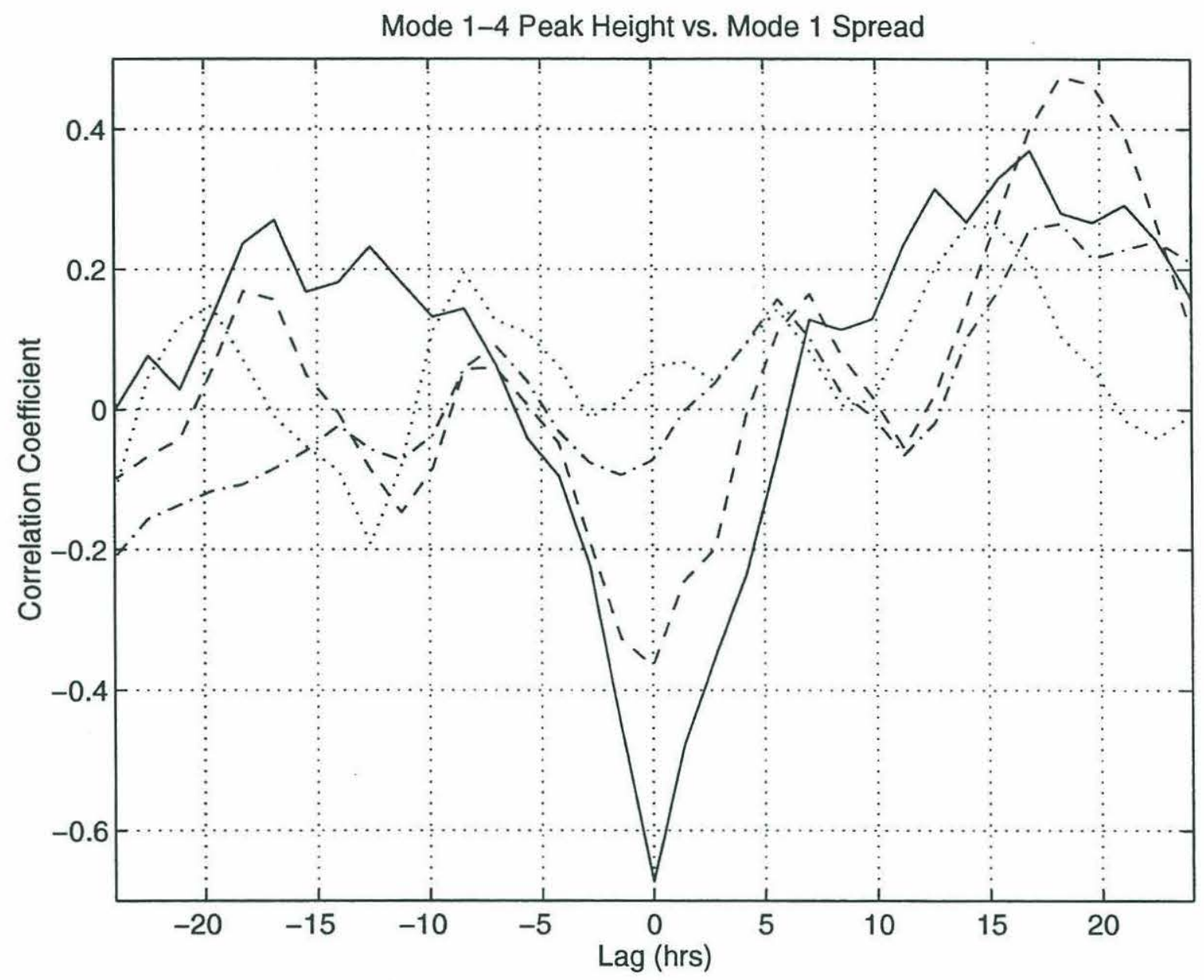

Figure 5-19: Cross-correlations of $1.4 \mathrm{hr}$ averaged peak height fluctuations for mode 1 (solid), 2 (dashed), 3 (dot-dashed), and 4 (dotted) with the corresponding $1.4 \mathrm{hr}$ averaged mode 1 IQR (spread) levels.

\begin{tabular}{|c|c|c|c|c|}
\hline \multicolumn{5}{|c|}{ Zero-Lag Cross-Correlations } \\
\hline Spread & \multicolumn{4}{|c|}{ Peak Height Mode } \\
\cline { 2 - 5 } Mode & 1 & 2 & \multicolumn{1}{c|}{3} & \multicolumn{1}{c|}{} \\
\hline 1 & -.67 & -.36 & -.07 & .06 \\
2 & -.27 & -.72 & -.35 & -.17 \\
3 & .10 & -.46 & -.62 & -.21 \\
4 & .30 & .04 & .23 & -.26 \\
\hline
\end{tabular}

Table 5.2: Zero-lag cross correlations between 1.4 hour bin averaged mode 1-4 peak heights and mode 1-4 mean spread levels. 


\subsection{The Transition to Real Data}

A problem with conversion from simulations to real data analysis is a loss of the knowledge of the exact location of the PAM1 arrival. In the simulation analysis this location was easily calculated, because the environments were deterministic. In real data, the location of this arrival is unknown. Furthermore, its location will change from sequence to sequence. This movement is referred to as the wander of the mode 1 arrival. Some small amounts of wander may occur over short time scales, but wander signals are generally dominated by large scale changes in temperature or current. These carry with them longer time scales than the soliton scattering that dominates the spread and bias of the mode 1 arrival. In our shallow waveguide, mode 1 energy is confined to the cold slow region below the pycnocline (see Figures 4-6 and 4-7), so passage of a soliton tends to push mode 1 deeper. The deepening of the mode 1 shape almost exactly compensates for a simultaneous increase in mean sound-speed, such that the mode 1 group velocity is relatively insensitive to the solitons in the waveguide. This translates to a very low mode 1 soliton wander signal. In our SIA model, a change from zero to 30 solitons caused only about a $1 \mathrm{~ms}$ drop in the PAM1 travel time. This compensating behavior combined with the fact that the most energetic linear internal waves are confined to the upper water column, should keep most sources of wander, at soliton time-scales, small compared to the bias signals caused by soliton induced scattering. The difference in time scales between the major sources of wander and major sources of bias provides the potential for separating them!

As illustrated by the relationship between the peak arrival distribution leading edge and the PAM1 arrival curve plotted in Figure 5-15, estimating the PAM1 arrival time should not be too difficult. The histograms of SIA model peak positions (Figure 5-6) provide some further insight; the PAM1 arrival is always in one of the first three bins, usually the second, so for a group of 100 random realizations of a simulated waveguide, the earliest peak sample time (plus one sample) may be a reasonably 
good estimate for the PAM1 arrival.

The applicability of this estimate to the wander of real mode 1 arrivals does not follow directly. The SWARM data has 22 sequences every six minutes, so approximately 200 sequences are available each hour. However, many of the sequences will be correlated. The measured correlation times are between 70 and 90 seconds, so our 200 samples of actual data may only be equivalent 15 or 20 random realizations. However, this should be sufficient; PAM1 estimation is clearly possible with the 10 samples per hour data rate shown in Figure 5-15.

Estimating the pseudo-adiabatic arrival times (i.e. wander) for higher modes will be more problematic. This makes the determination of bias levels for higher modes difficult and imprecise. However, the measures of spread used in the simulated data are not directly dependent on knowing either the wander or the bias, so the transition to real data analysis for this variable is straightforward. 


\section{Chapter 6}

\section{Analysis of Real Data}

\section{1 $400 \mathrm{~Hz}$ Mode 1 Arrival Analysis}

\subsubsection{Peak Arrival Time}

The nature of the SWARM waveguide is such that mode 1 generally has the highest group velocity, making the PAM1 arrival the earliest possible arrival for most transmissions. There are exceptions. During the early hours of day 212, at the WVLA, mode 2 has a higher group velocity than mode 1; if and when this is the case for the majority of the waveguide, the PAM2 arrival will precede the PAM1 arrival, allowing for some positively biased mode 1 energy, as seen in Figure 5-15.

The distribution of SWARM mode 1 peak arrival times shown in Figure 6-1 has a fairly clean leading edge; there is little or no indication of negatively biased peaks. This strongly suggests that the PAM1 arrival was the earliest arrival over most, if not all, of the period under evaluation. The pattern of the PAM1 arrival time (i.e. the leading edge) shows a clear semidiurnal tidal signal, as well as significant wander at even lower frequencies. The two strongest and most probable sources for these fluctuations are barotropic tidal currents along the path between the source and receiver and the tidal advection of deeper warm water (e.g. the foot of the shelfbreak 


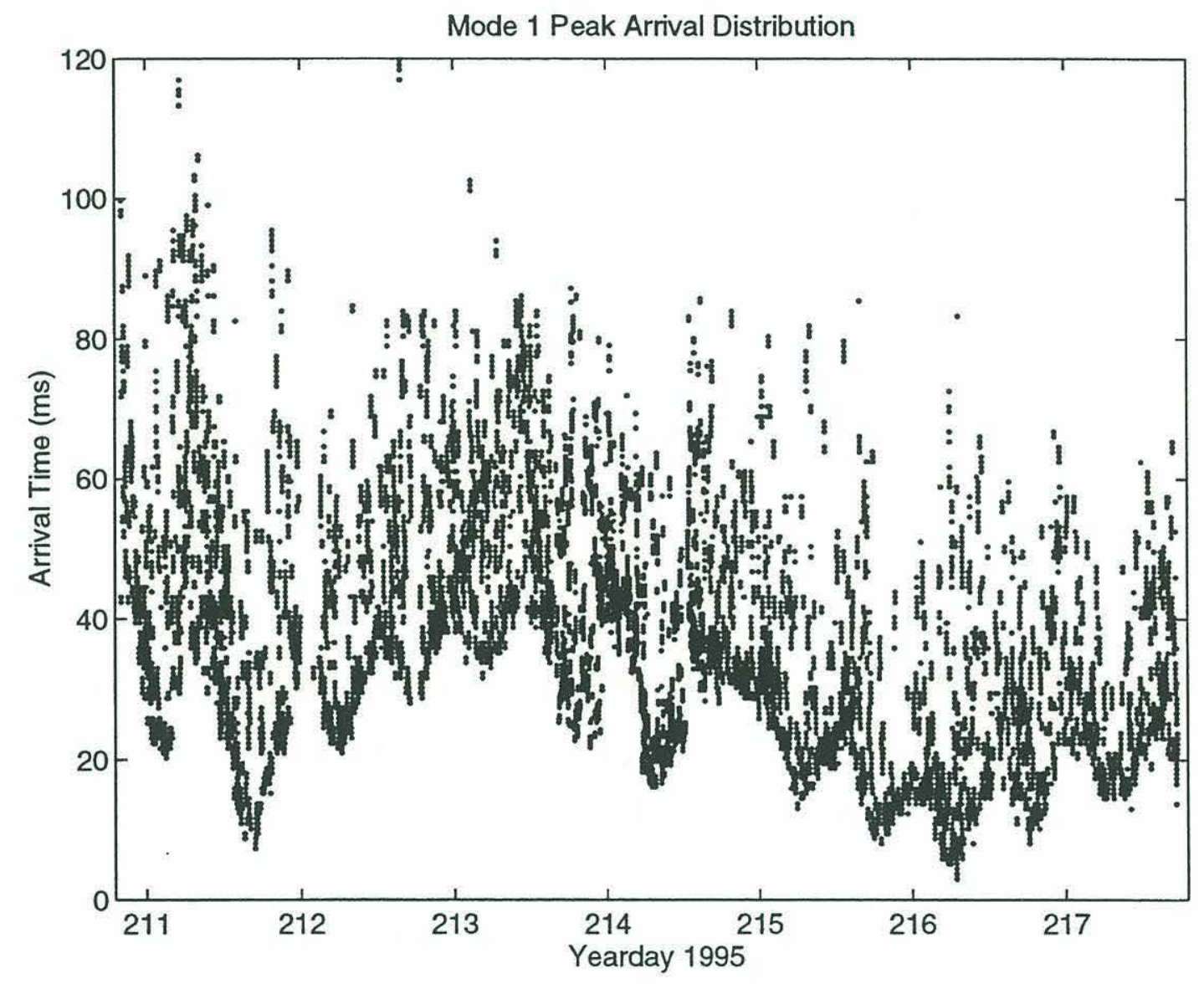

Figure 6-1: Distribution of SWARM Mode 1 Peak arrival times. The zero reference is at an arrival time of 21.985 seconds. The plotted transmissions occurred every six minutes, with each transmission producing 22 sequence arrivals over a two minute period. The location of the highest peak in each sequence is plotted. Though not evident in most cases, there are gaps in the record that range from single lost transmissions to occasional larger gaps of up to two hours in duration. 
front) in and out of the acoustic path.

Near the WVLA, Mode 1 energy is for the most part confined to the slower sound speed regions in the 40 to $60 \mathrm{~m}$ depth band (see Figure 4-7). An upward looking Acoustic Doppler Current Profiler (ADCP) moored near the WVLA in $75 \mathrm{~m}$ of water provides a measure of the currents in the region. Taking the average current in five $4 \mathrm{~m}$ depth bins between 39 and $59 \mathrm{~m}$ depth, low-pass filtering, and integrating the effects on travel time over the entire $32,000 \mathrm{~m}$ acoustic path (assuming range independent currents) yields the solid curve shown in Figure 6-2. Using 14 bins between 11 and 67 $\mathrm{m}$ depth produces the dotted line. The similarity between the two curves shows that the vast majority of the current signal in the depth band of interest (solid curve) is barotropic (baroclinic currents from solitons are removed by the lowpass filter). The barotropic current clearly accounts for some of the semidiurnal PAM1 wander, but it contains negligible lower frequency signals.

Subtracting the current signal from the mode 1 peak arrival times yields the residual distribution of peak arrival times plotted in Figure 6-3. The residual mode 1 wander at the semidiurnal tidal frequency is substantial, and a good portion of it can be correlated with temperature fluctuations in the vicinity of the WVLA. These were logged in deep water thermistor records from the WVLA and three dedicated thermistor strings moored in a cluster $4.5 \mathrm{~km}$ shoreward. The solid curve in Figure 6-3 is obtained from the average change in sound speed seen at seven of these thermistors applied over a distance of $8000 \mathrm{~m}$. The seven records used (three from the WVLA, two from one dedicated string, and one each from the other two) were in the depth band of interest for mode $1(40-60 \mathrm{~m})$ and judged to be reliable. The foot of the shelfbreak front, which has been observed (in CTD profiles) to advect across the position of the WVLA, seems a likely source of these temperature fluctuations.

The actual range between the thermistors is only $4500 \mathrm{~m}$, but the effects may be reasonably extended further, and the choice of $8000 \mathrm{~m}$ provides a level of predicted variance close to that observed in the acoustic data. However, the PAM1 wander 


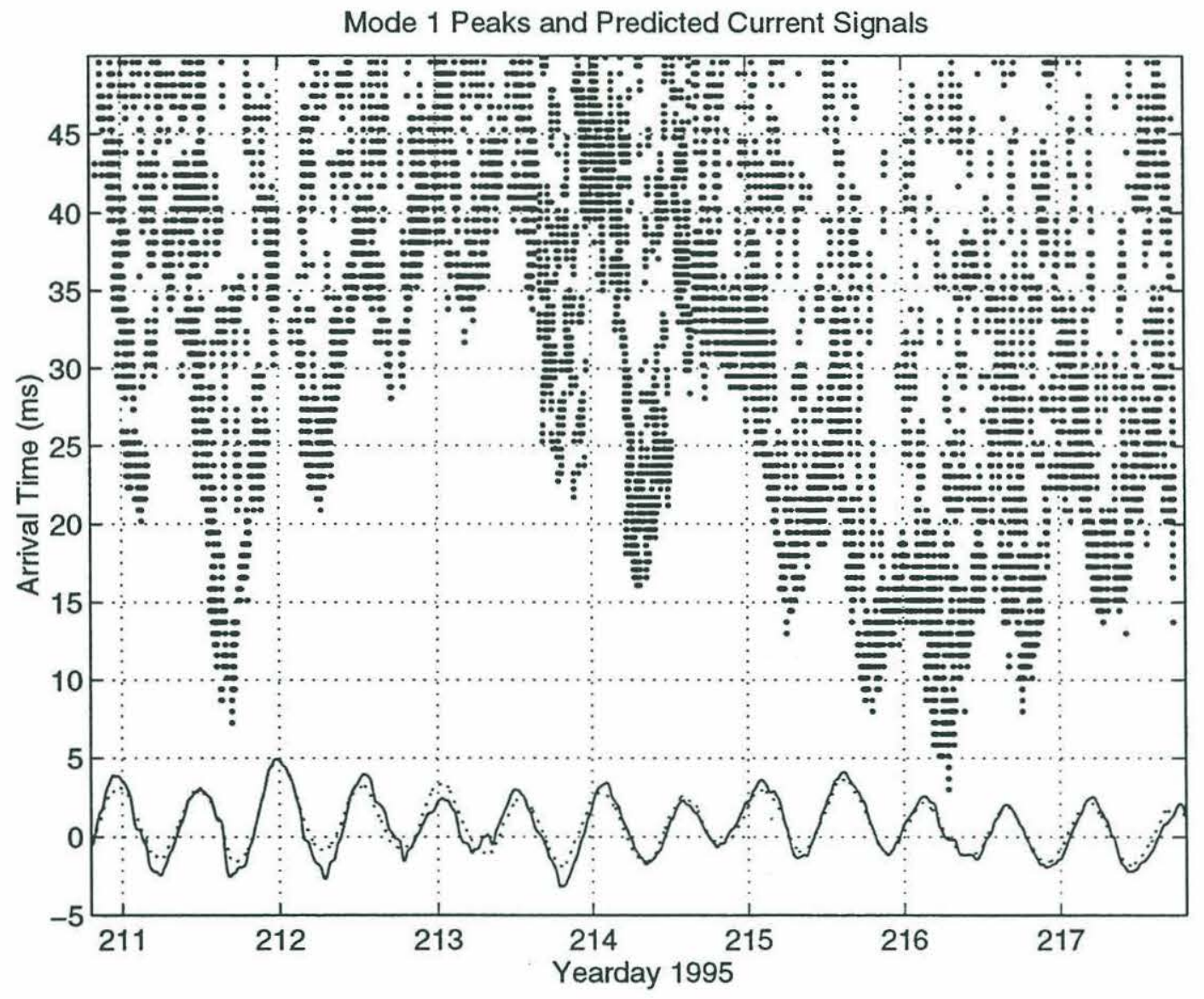

Figure 6-2: Predicted current effects on PAM1 arrival time plotted with the distribution of peak arrival times shown in Figure 6-1. The solid curve is based on the mean current between 39 and $59 \mathrm{~m}$ depth and the dotted curve is based on the average current between 11 and $67 \mathrm{~m}$ depth. 


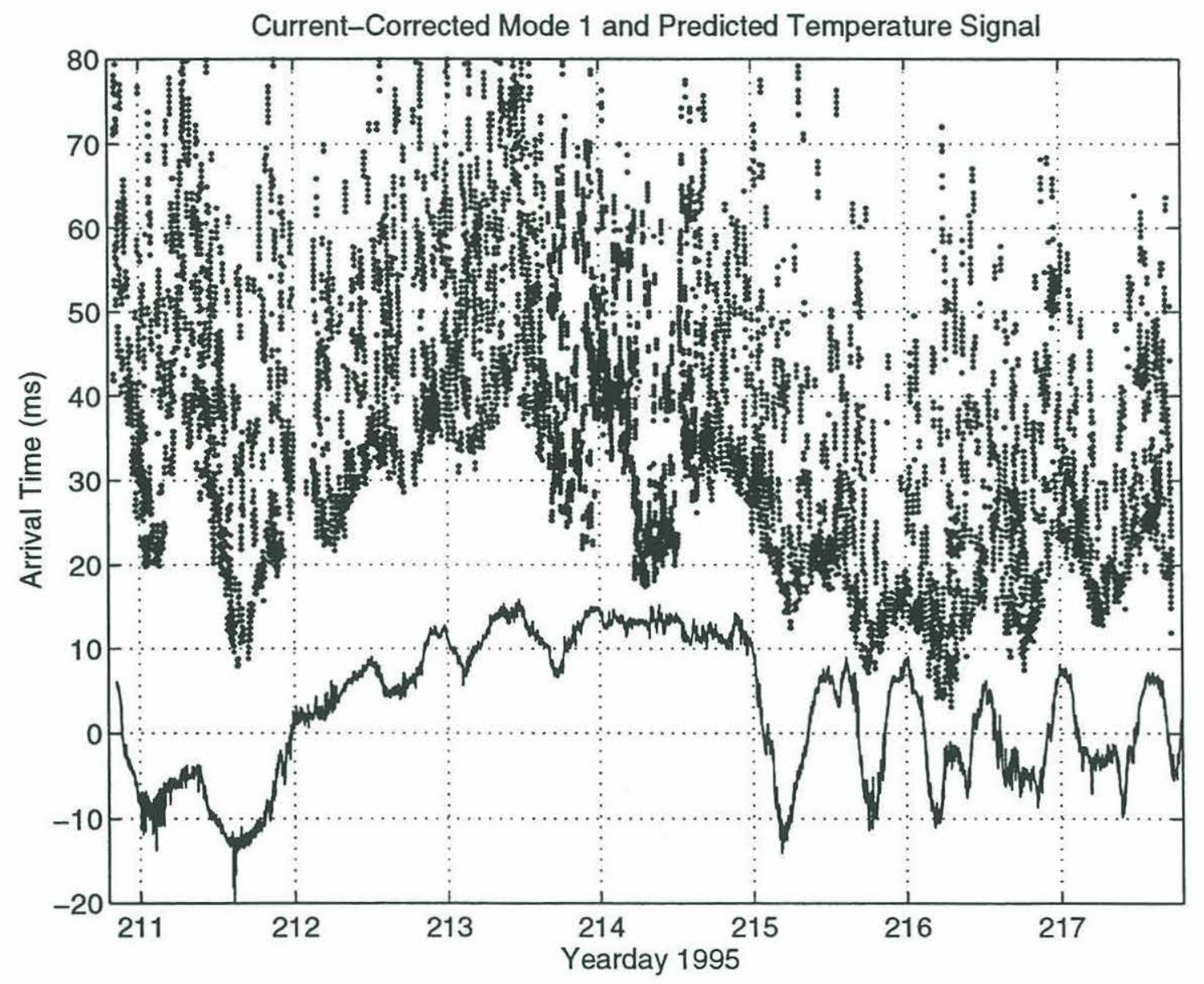

Figure 6-3: Predicted temperature fluctuation effects on PAM1 arrival time (solid curve) plotted with the distribution of "current-corrected" peak arrival times. The solid curve is zero mean (by definition) and offset from the data for convenience. 


\section{Mode 1 Peak Arrival Bias}
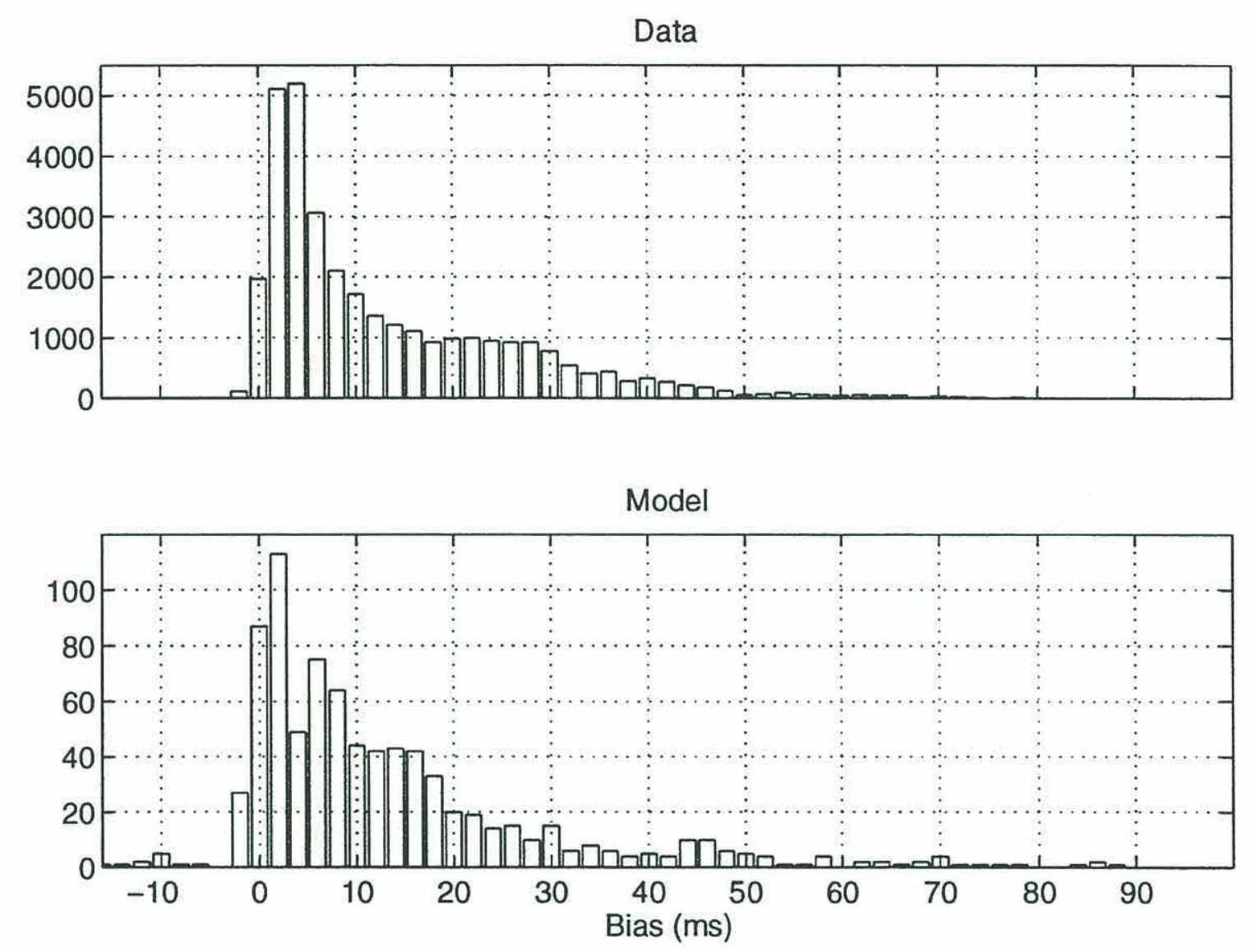

Figure 6-4: Mode 1 peak arrival time bias histograms for the data shown in Figure 6-1 and the model distribution shown Figure 5-15. The bias measurements are relative to estimated PAM1 arrival times (i.e. the leading edge) for the data and calculated PAM1 arrival times for the model.

occurring on yearday 214 is a clear indicator that large temperature fluctuations are not restricted to the area around the WVLA, and it is likely that changes in temperature along the entire $32 \mathrm{~km}$ path contribute at various times in varying degrees to the mode 1 wander signal at the WVLA.

Regardless of its source, the wander signal evident in Figure 6-1 can be estimated and subtracted from the distribution of peak arrival times. A histogram of the resulting peak arrival "bias" levels is shown in the upper frame of Figure 6-4. A mode 1 peak arrival time bias histogram obtained from the "propagated thermistor model" 
in Chapter 5 is shown in the lower frame of the same figure. The histograms are quite similar, even though the "Data" is based on over 32,000 arrivals and the "Model" uses only 816. Not surprisingly, the "Model" histogram is not quite as smooth; it also shows a negatively biased pocket that stems from the period when the modeled path averaged group speed for mode 2 was higher than mode 1 (see Figure 5-15).

\subsubsection{Arrival Spread}

The range and variability of positive bias seen in the Figure 6-1 peak arrival distribution is indicative of significant time spreading in the mode 1 arrival patterns. Spread is perhaps the simplest quantifying measure of signal distortion, and as such, it is a clear indicator of the mode scattering produced by the waveguide.

Spread is conceptually simple, yet, as discussed in Chapter 5, the choice of which mathematical definition is "best" to use is somewhat subjective. The four measures that will be considered here are: (1) the IQR of the arrival envelope; (2) the standard deviation of the arrival envelope; (3) the absolute value of the peak minus the mean arrival time; and (4) the standard deviation of the peak times in a 22 sequence transmission. This list is obviously not exhaustive but is a good selection of the possibilities.

Using method one, the IQR, several different bin averages of the WVLA mode 1 arrival spread are shown in Figure 6-5. The upper frame is a plot of the average spread

for each transmission, while lower frame includes five different bin averages ranging from 1.4 to 4.1 hours for comparison. The upper frame of Figure 6-5 reveals significant variability over a time scales ranging from several minutes up to semidiurnal tidal oscillations. The tidal oscillations, among some other low frequency signals, are also evident in the longer averages shown in the lower frame.

The second method, standard deviation, is very similar to the first; the main difference being in the sensitivity to outlying energy. The third method, absolute value of peak minus mean, strictly speaking, is more a measure of "nongaussianess" 

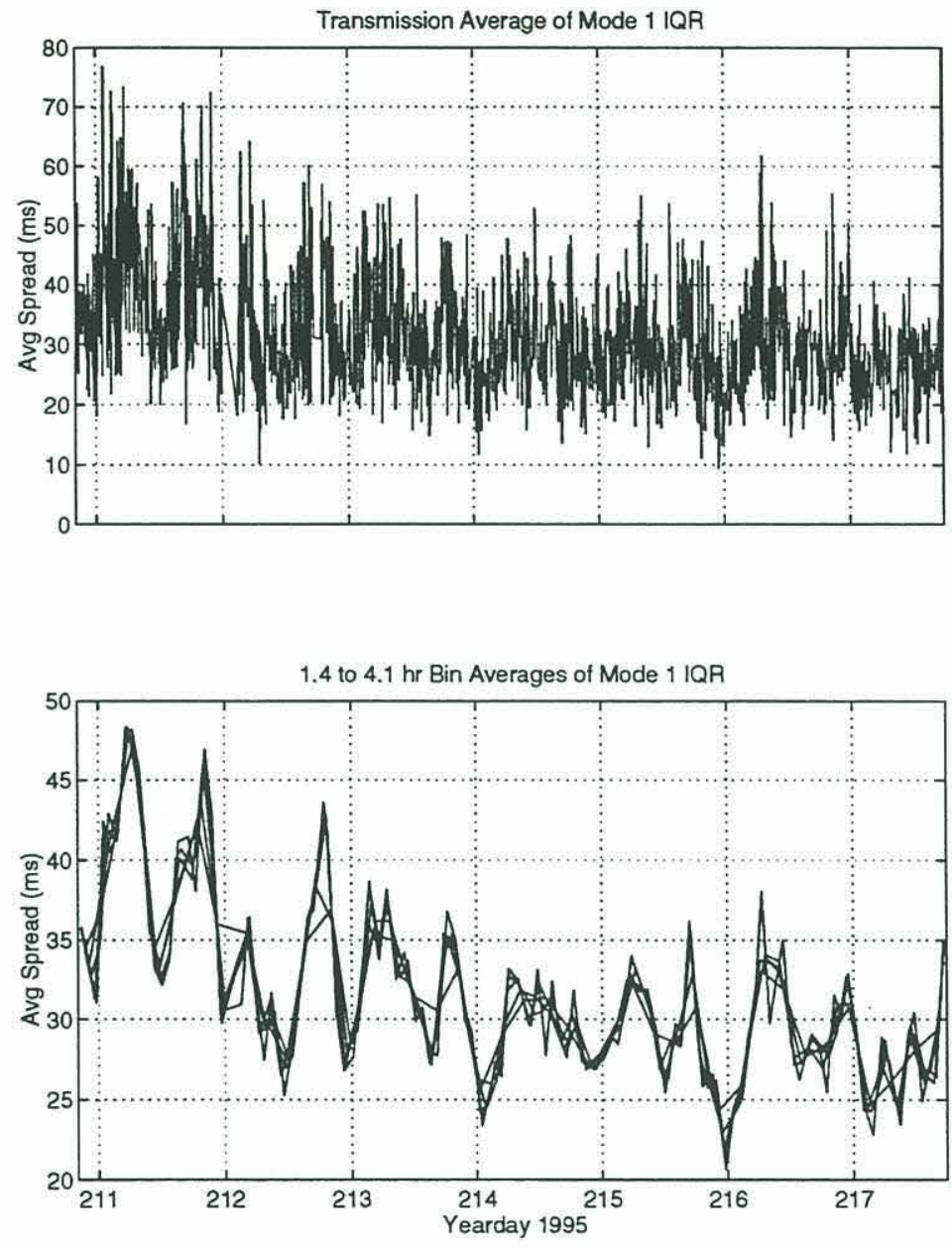

Figure 6-5: Mode 1 arrival spread, as measured by the IQR of the arrival envelope (unscattered IQR is about $7.2 \mathrm{~ms}$ ). The upper frame is plot of the spread when averaged over each transmission ( 22 sequences per transmission). The lower frame shows bin averaged spreads for five bin widths ranging from 1.4 to $4.1 \mathrm{hrs}$. 
than a measure of spread, but as we will see, these usually go hand-in-hand. Whereas the first three methods require evaluation of the arrival envelope, the fourth method, standard deviation of peak times, only requires the peak arrival times to be recorded. The tradeoff for this is that only one measurement can be obtained per transmission, as opposed to 22 per transmission for the other three.

Applying each of these methods to the mode 1 arrival records from SWARM produces varying degrees of similarity between methods. Using 1.4 hour bin averages (see Figure 6-5) as benchmarks, the following matrix of zero-lag cross-correlation values can be constructed:

$$
\left[\begin{array}{rrrr}
1.00 & .97 & .85 & .57 \\
& 1.00 & .82 & .60 \\
& & 1.00 & .29 \\
& & & 1.00
\end{array}\right]
$$

As expected, the correlation is the strongest between methods one and two, and the other combinations show limited similarity. The IQR will remain the primary method by which spread is measured.

\section{Tidal Signals in Spread Spectra}

Figure 6-6 contains the spectra of the spread time histories in the lower frame of Figure 6-5. The spectra confirm the existence of significant variability near the semidiurnal tidal frequency. This tidal variability is consistent with our understanding of the most probable source of the spread in the arrivals: internal wave solitons.

The relationship between tides and soliton formation appears to be strong, but it is not strict and exclusive, and it is certainly not linear; the numbers and sizes of solitons, as well as the extent of solibores (internal tide depressions) they are often associated with, can change dramatically from one tidal cycle to the next. This, coupled with multiple soliton-generation sites and the nonlinear relationships between 


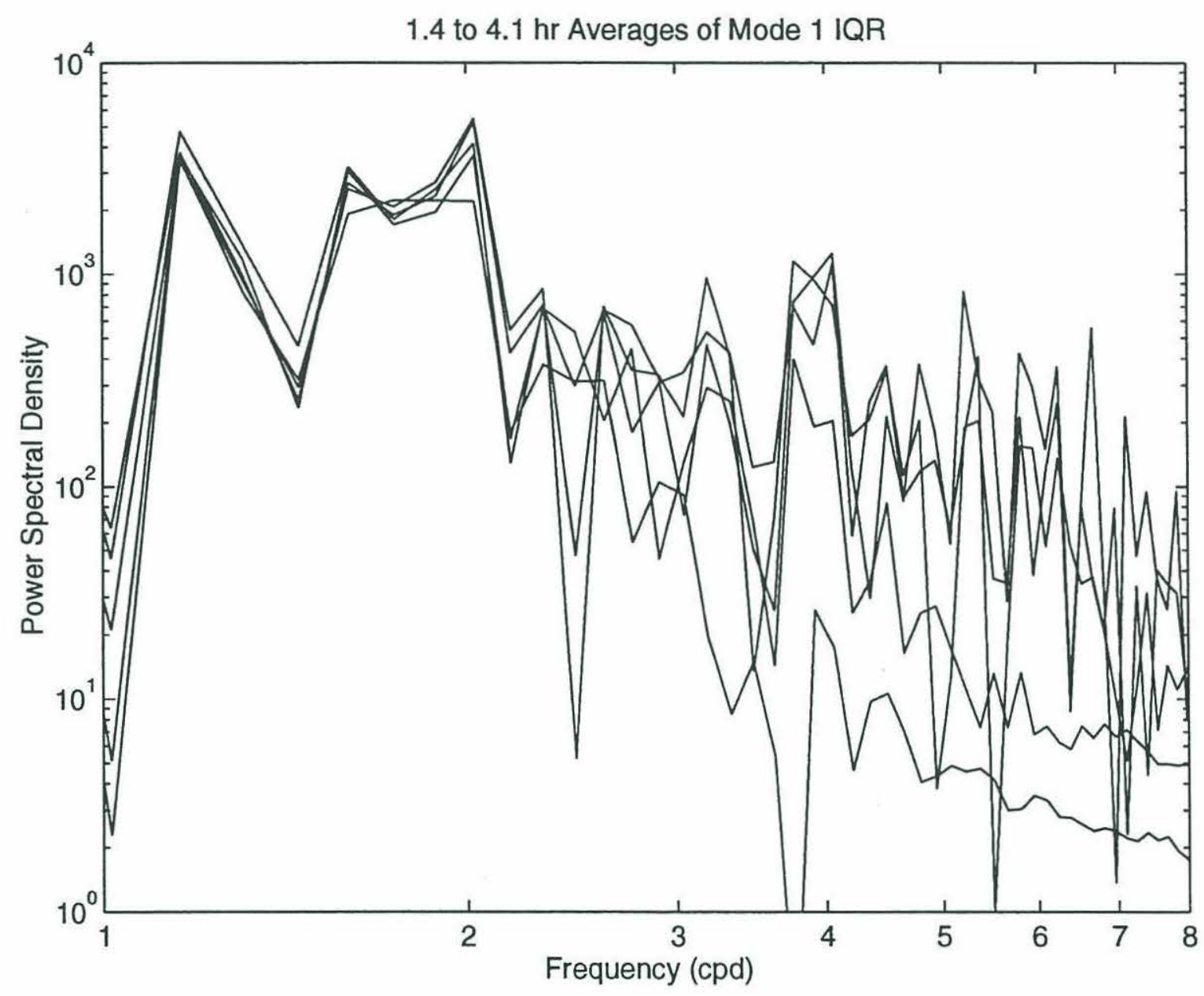

Figure 6-6: Power Spectral Density of the bin averaged spreads shown in the lower frame of Figure 6-5. 


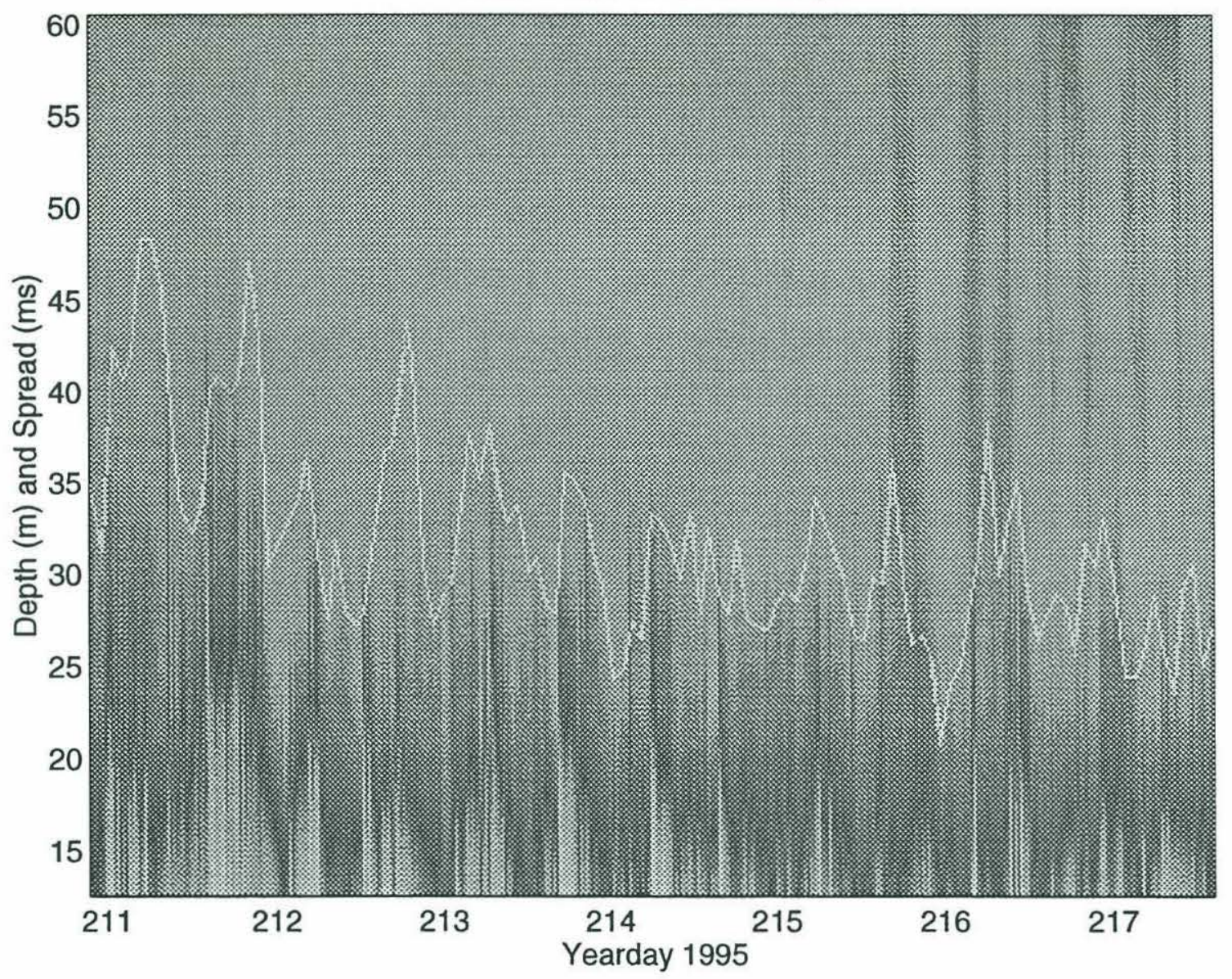

Figure 6-7: WVLA temperature as function of time and depth overlaid by the $1.4 \mathrm{hr}$ bin averaged IQR of mode 1 arrivals at the WVLA. The temperatures in this figure range from 7.2 to 26.5 degrees $\mathrm{C}$.

spread and soliton density, accounts for the broadband nature of the low frequency spread spectrum.

Figure 6-7 illustrates both the variability of the soliton packets from cycle to cycle and the variability in the degree to which mean spread levels are controlled by the density of solitons in the near receiver half of the waveguide.

This figure is similar to its simulation counterpart, the upper frame of Figure 5-17, but it differs in several important considerations: first, the mean spread levels are from actual arrivals, not simulations; second, the mean spread levels are calculated using 
upwards of 100 sequences per hour (depending on the number of gaps in the data), instead of just 10 per hour in the simulations; third, the bin width for averaging is 1.4 hrs, vice 30 minutes; and finally, the temperature profiles in the area 670 minutes to the left of the spread reading in question are only an indication of what the waveguide may have looked like at the time of the transmission.

Both figures show some degree of correlation between spread levels and soliton activity near the receiver, but it is difficult to quantify. If the standard deviation of the temperature at $22.5 \mathrm{~m}$ depth over the previous $4.3 \mathrm{hrs}$ is again used as a crude measure of soliton activity near the receiver, then we can see a clearer picture of the correlation, at least for the first half of the record, as shown in Figure 6-8. This figure also shows that there is some correlation between the mean spread levels from the simulations and the mean spread levels found in the data. Obviously the similarities could be coincidental, as the correlation is not strong and consistent, but given the nonlinear spread response to soliton density and the unavoidable differences between the modeled and actual waveguides, one cannot expect extremely close agreement. The mere fact that the behaviors show some limited agreement may not be solid proof of the veracity of the model, but neither are the differences evidence against that proposition!

\subsubsection{Peak Arrival Intensity}

Mode 1 arrivals with large amounts of distortion, gained that distortion through mode-coupling interactions. Greater amounts of spread generally indicate greater percentages of subarrivals having spent large amounts of time in higher modes. The act of time-spreading alone will reduce the peak intensity of a mode 1 arrival, and this, in combination with travel in higher modes causing more attenuation, should create a signal in the mean peak intensity of the arrivals that is inversely proportional to mean spread in the arrivals. A comparison between average levels of spread and relative intensity loss of mode 1 arrivals is made in Figure 6-9. The degree to which 
Mode 1 IQR (ms) and T-String STD (centidegrees)
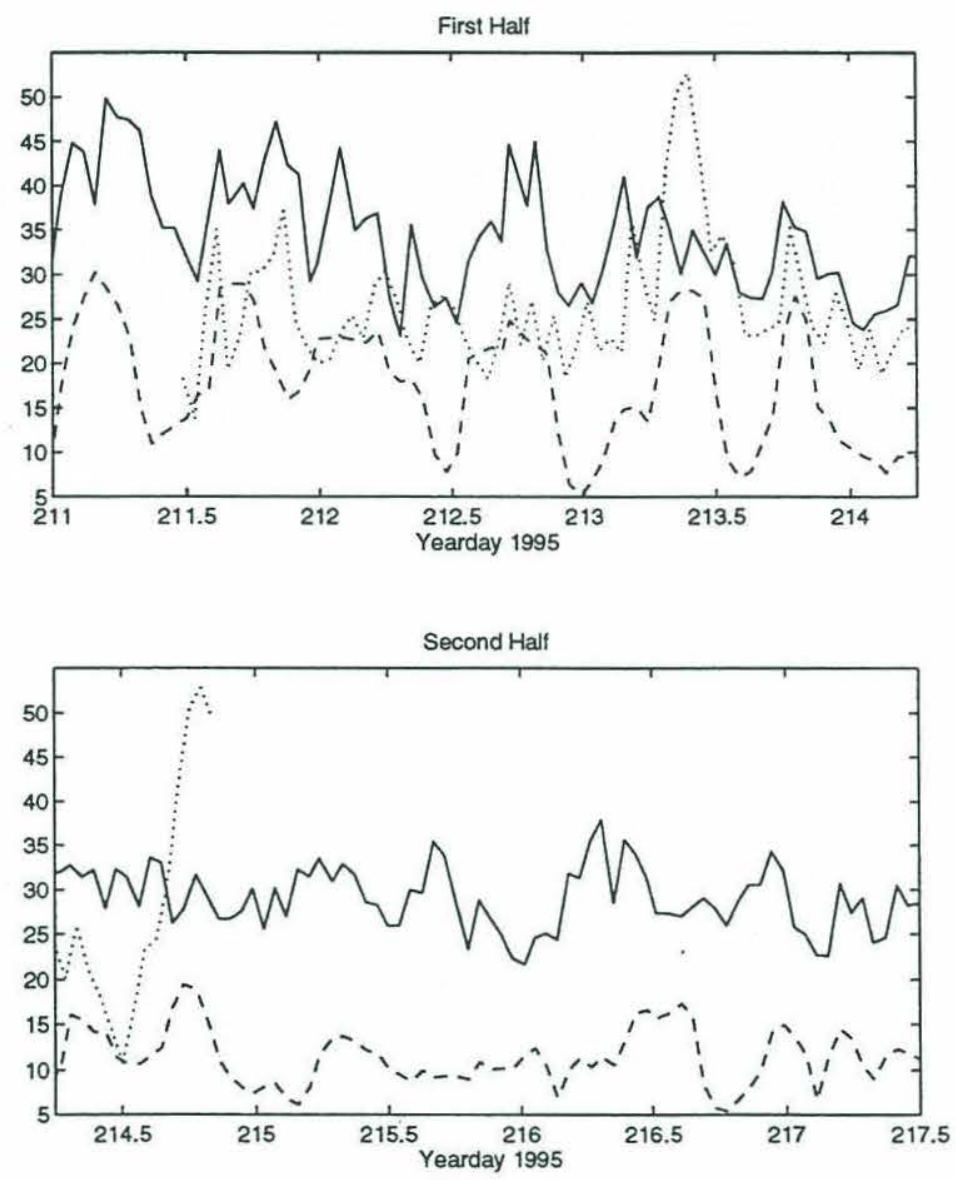

Figure 6-8: A comparison of 1 hour bin averaged IQR levels for the SWARM data (solid line), the corresponding propagated thermistor string model (dotted line), and the Standard Deviation of the Temperature at $22.5 \mathrm{~m}$ depth over the previous $4.3 \mathrm{hrs}$ (dashed line). The standard deviation of the temperature is in tenths of a degree $\mathrm{C}$ to facilitate comparison with the IQR levels in milliseconds. 

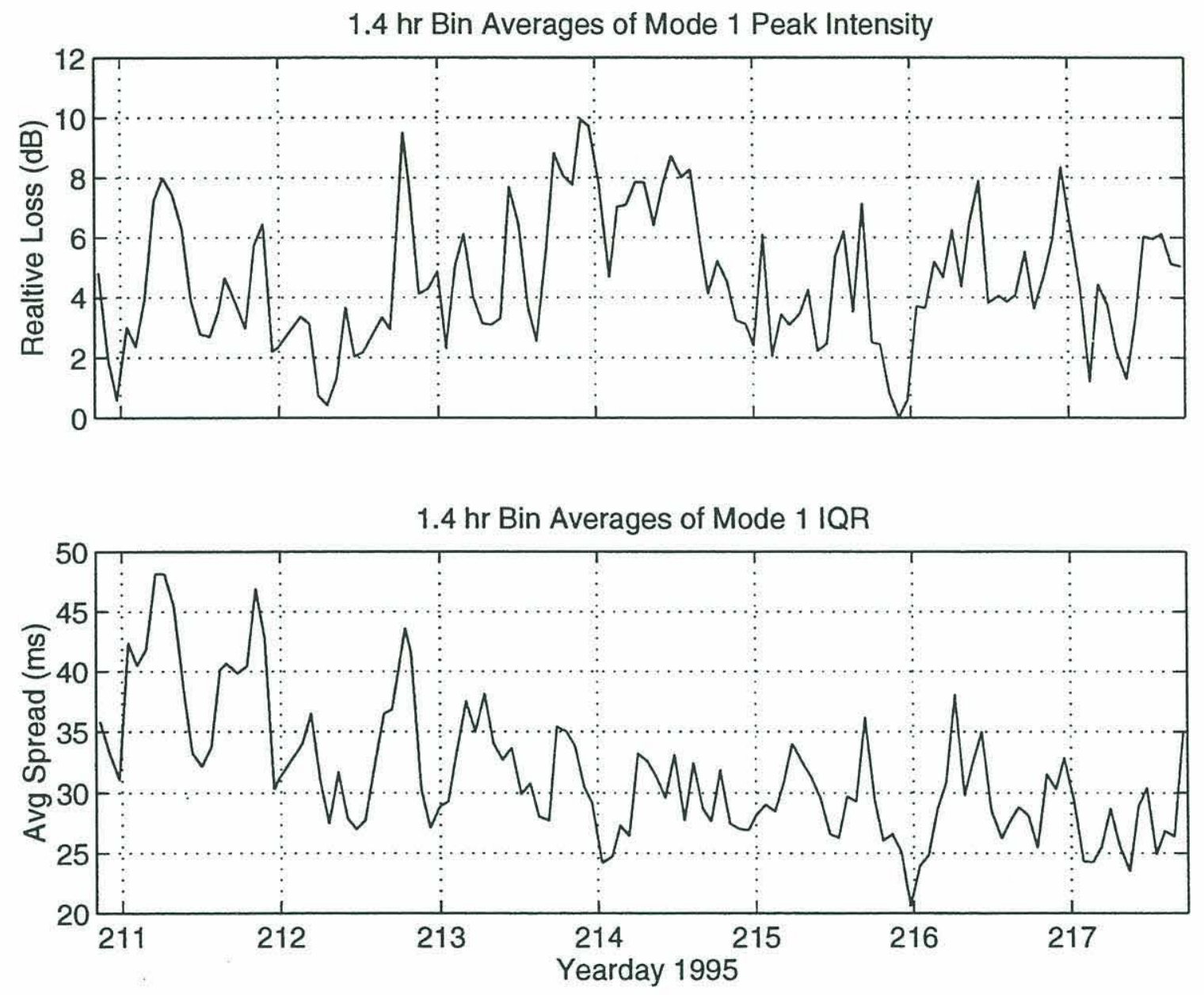

Figure 6-9: A comparison of $1.4 \mathrm{hr}$ averaged peak intensity fluctuations and the corresponding $1.4 \mathrm{hr}$ averaged IQR levels for the SWARM data. The peak intensity fluctuations are plotted in terms of relative loss to provide a signal that is in phase with the spread fluctuations. 
these correlate (the peak cross-correlation is .29 at zero lag) is an indication of the strength of the link between mode 1 spread and peak intensity. Much of the near-tidal variation in both statistics appears to be in phase, but the majority of the remaining variance does not appear to correlate. While scattering is probably the dominant influence on spread and a major source of intensity fluctuations, Figure 6-9 shows the relationships to be nonlinear and/or the influence of other factors to be substantial.

The mode excitation at the source, for example, can have variability over a wide range of time scales. The effect of this on peak intensity is somewhat predictable, but the influence on spread is situational. Conversely, as observed in the Chapter 5 simulations, shifts in relative group speed can influence spread without necessarily affecting peak intensity one way or the other.

\subsubsection{Higher Mode Statistics}

\section{Peak Height}

An interesting comparison can be made using the mean peak-height statistics, shown in Figure 6-10, for the first four modes. The zero-lag cross-correlation coefficients between pairs of these peak height statistics are compared with the same statistics for simulated data in Table 6-1. The peaks fluctuate in height coherently to a great degree; roughly half the variance is coherent between modes. The simulated data is the same shown in Figures 5-14 and 5-15, and the cross-talk contamination is simulated with incoherent mixing based on the "Average" filter performance matrix shown in section 3.3.2. The increases in the statistical correlations caused by this mixing are in some cases significant, and, no doubt, some part of the correlation seen in the SWARM data is also from cross-talk. The zero-lag cross correlations between the "unmixed" simulated data and the "cross-talk" simulated data are 0.98, 0.98, 0.92 , and 0.96 for modes 1 to 4 respectively.

Figure 6-10 shows that much of the similarity in the SWARM data is from what appear to be coherent tidal fluctuations in mode excitation and/or attenuation. The 


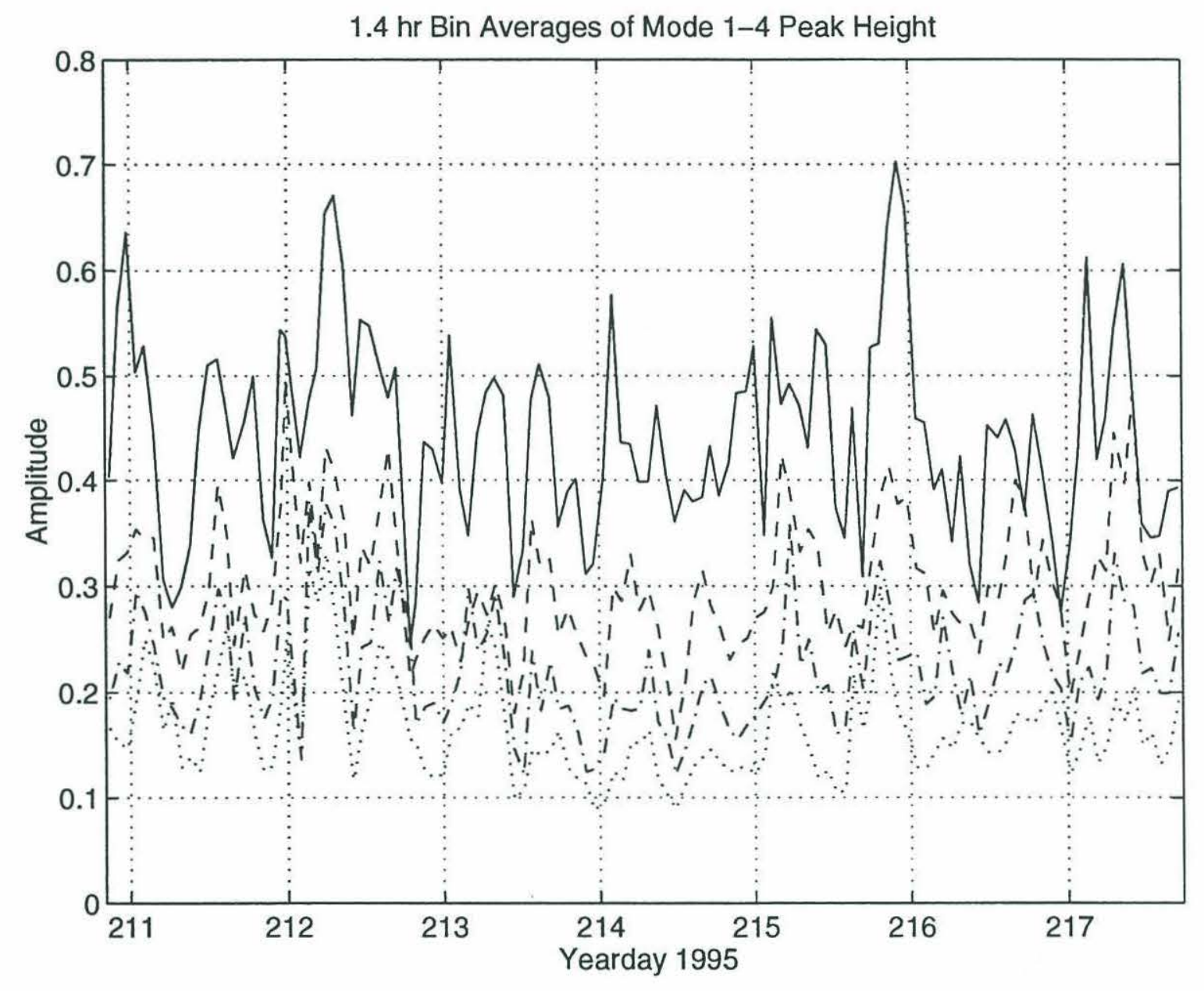

Figure 6-10: A comparison of $1.4 \mathrm{hr}$ averaged peak-height fluctuations for the first four modes of the SWARM data. Mode 1 is solid, mode 2 is dot-dashed, mode 3 is dashed, and mode 4 is dotted. 


\begin{tabular}{|l|c|c|c|}
\hline \multicolumn{4}{|c|}{ Bin Avg Peak Height } \\
\hline \multicolumn{3}{|c|}{ Zero-Lag Cross-Correlations } \\
\hline Mode & SWARM & \multicolumn{2}{|c|}{ Simulated Data } \\
\cline { 3 - 4 } Pair & Data & Unmixed & Cross-Talk \\
\hline $1: 2$ & .61 & .48 & .50 \\
$1: 3$ & .52 & .26 & .34 \\
$1: 4$ & .44 & .24 & .29 \\
$2: 3$ & .74 & .65 & .65 \\
$2: 4$ & .63 & .39 & .49 \\
$3: 4$ & .83 & .57 & .86 \\
\hline
\end{tabular}

Table 6.1: Zero-lag cross correlations of 1.4 hour bin averaged peak heights for the SWARM data and the simulated data of Chapter 5. The unmixed data (modes 1 and 2) are shown in Figures 5-14 and 5-15; the cross-talk contaminated is from the same source, with incoherent mixing based on the "Average" filter performance matrix shown in section 3.3.2.

tidal signal becomes more apparent and shifts in phase as the mode number increases.

\section{Peak Height and Spread Correlations}

Experience with the simulated transmissions in Chapter 5 and the existence of tidal fluctuations in the peak-height statistics suggests a probable correlation between spread and peak height statistics. Figure 6-11 shows the result of cross-correlations between mode 1 spread and mode 1-4 peak heights. Confirming our visual analysis of Figure 6-9, we see that peak height fluctuations for mode 1 have a negative a zero-lag correlation with mode 1 spread statistics. This is in agreement with simulations using the "propagated thermistor string" (see Figure 5-18). The correlation becomes positive and develops a tidal period as the mode number is increased; this is consistent with the concept of scattering being an energy sink for mode 1 and an energy source for higher modes.

The zero-lag cross-correlations between spread levels for modes 1-4 and peak heights for modes 1-4, tabulated in Table 6-2, are similar in magnitude and trend to those observed with the Chapter 5 simulations. The spread measures of this table 


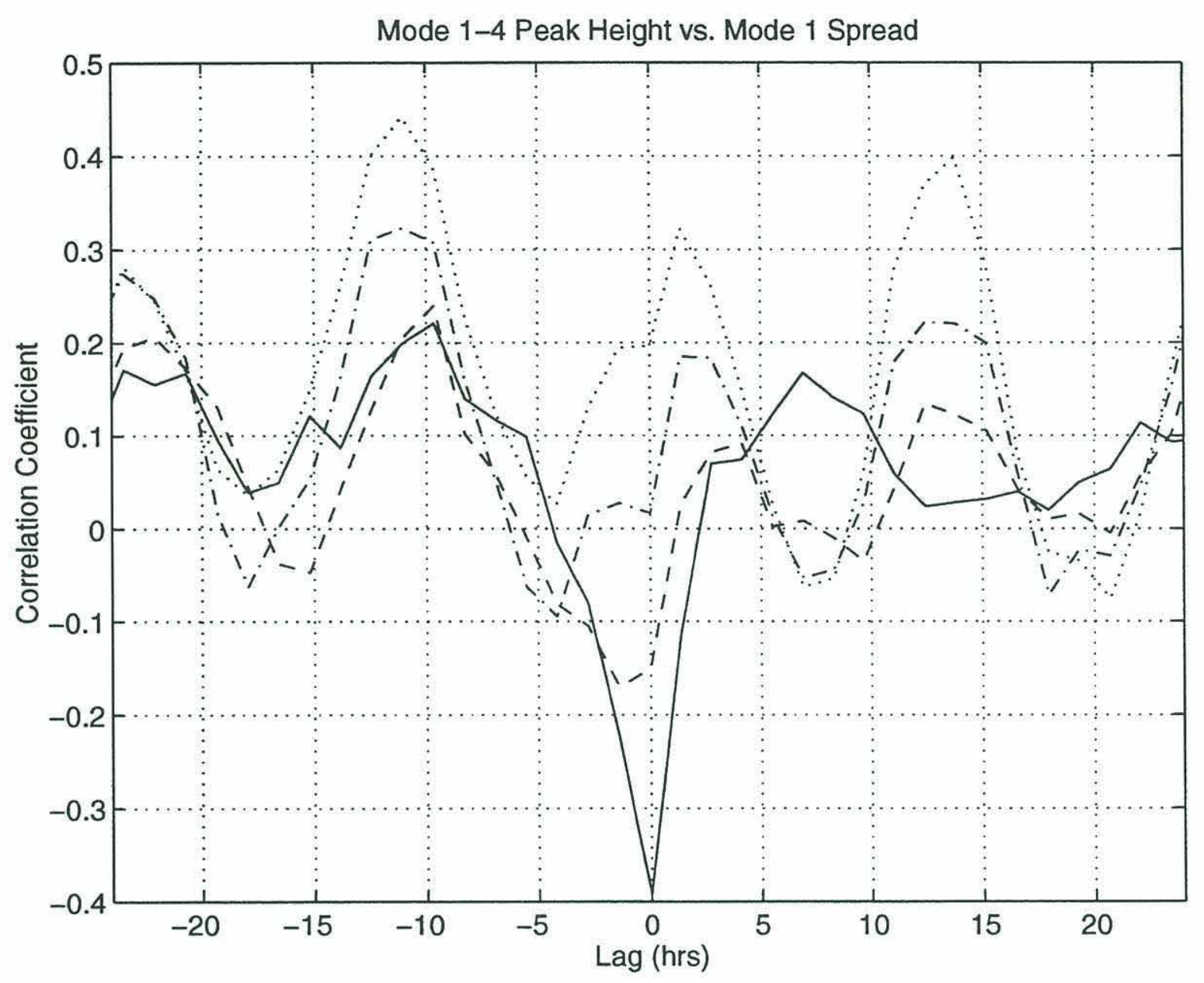

Figure 6-11: Cross-correlations of $1.4 \mathrm{hr}$ averaged peak height fluctuations for mode 1 (solid), 2 (dashed), 3 (dot-dashed), and 4 (dotted) with the corresponding $1.4 \mathrm{hr}$ averaged mode 1 IQR (spread) levels for the SWARM data.

\begin{tabular}{|c|c|c|c|c|}
\hline \multicolumn{5}{|c|}{ Zero-Lag Cross-Correlations } \\
\hline Spread & \multicolumn{4}{|c|}{ Peak Height Mode } \\
\cline { 2 - 5 } Mode & 1 & 2 & 3 & 4 \\
\hline 1 & -.53 & -.31 & -.21 & -.08 \\
2 & -.20 & -.48 & -.36 & -.26 \\
3 & -.11 & -.23 & -.35 & -.22 \\
4 & -.05 & -.27 & -.40 & -.43 \\
\hline
\end{tabular}

Table 6.2: Zero-lag cross correlations between 1.4 hour bin averaged mode 1-4 peak heights and mode 1-4 mean spread levels. The spread measurement employed for these cross-correlations is the standard deviation of peak arrival times over a transmission. 
are computed by method number four (as labeled above), the standard deviation of the peak arrival times taken over a transmission. This method, chosen primarily for its speed, produced a greater magnitude correlation between mode 1 spread and peak height than did the calculation using the IQR, shown in Figure 6-11.

\section{Peak Arrival Time}

Peak arrival scattergrams for the higher modes look similar to the mode 1 scattergram shown in Figure 6-1. The scattergram data for the first four modes are summarized, by way of their leading edges and bin averaged mean arrival times, in the plots shown in Figure 6-12. Although somewhat busy, this figure shows still another statistic with a strong tidal influence. The mean mode 4 arrival time has a definite tidal influence that is over and above the tidal modulation of the PAM1 arrival (i.e. the bumps in the latest dotted line have larger amplitudes than the bumps in the earliest solid line). These mean mode 4 arrivals are also negatively biased relative to the predicted PAM4 arrival, because they are generally within $80 \mathrm{~ms}$ of the mode 1 leading edge ( $80 \mathrm{~ms}$ being the average difference between computer simulated PAM1 and PAM4 arrivals).

\section{2 $224 \mathrm{~Hz}$ Arrival Analysis}

The $224 \mathrm{~Hz}$ tomography source, moored about $1000 \mathrm{~m}$ farther up the shelf, provides a second source of acoustic arrivals at the WVLA. The narrower bandwidth of the $224 \mathrm{~Hz}$ source yields a pulse width of about $60 \mathrm{~ms}$ versus the $10 \mathrm{~ms}$ pulse width of the $400 \mathrm{~Hz}$ signal.

\subsubsection{Peak Arrival Time}

The wider pulse width of the $224 \mathrm{~Hz}$ source no doubt contributes to the somewhat fuzzy distribution of $224 \mathrm{~Hz}$ mode 1 peak arrival times seen in Figure 6-13. It is 


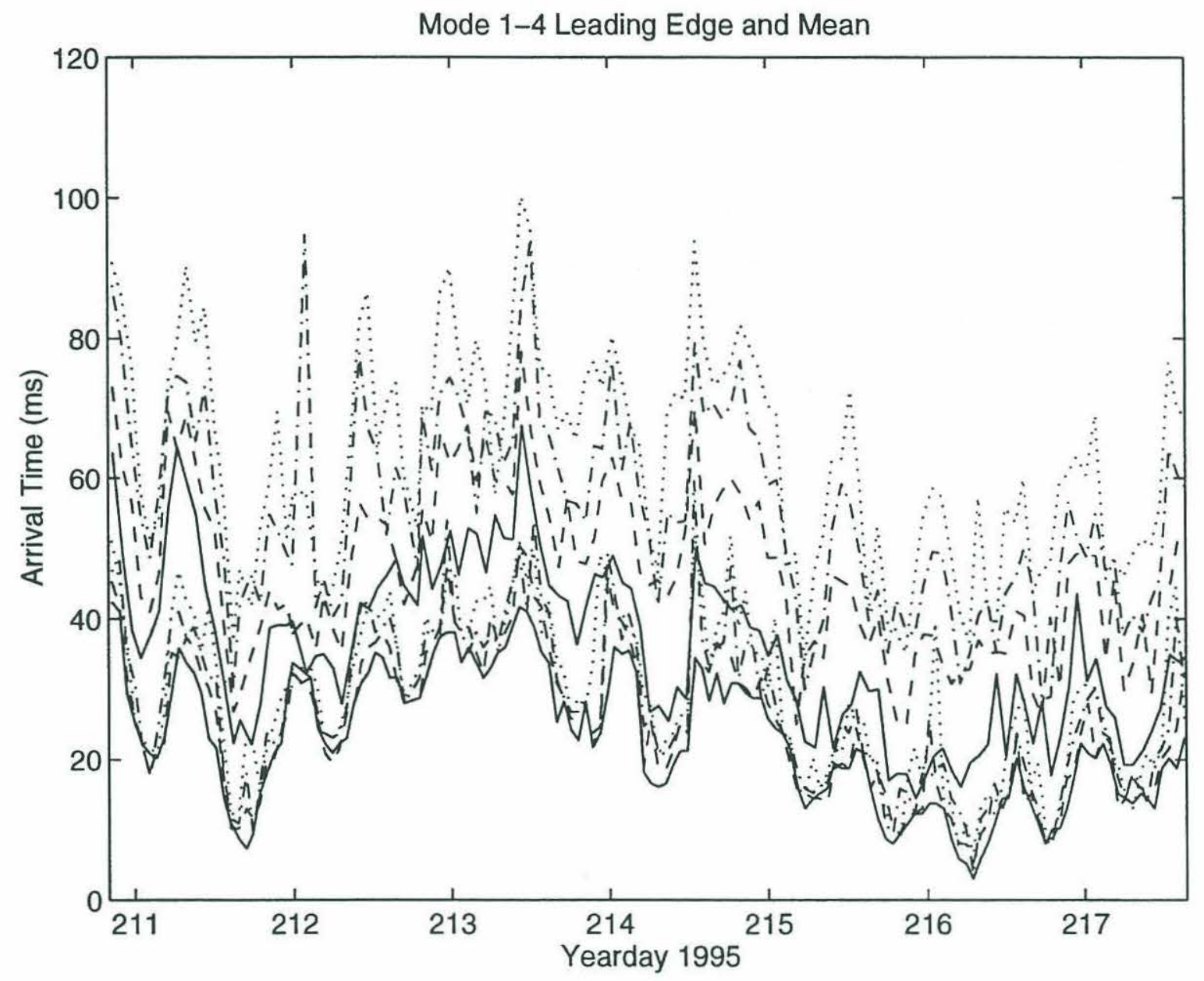

Figure 6-12: Mode 1 (solid), mode 2 (dashed), mode 3 (dot-dashed), and Mode 4 (dotted) peak arrival time leading edge (connects earliest peak arrivals in $1.1 \mathrm{hr}$ bins) and $1.4 \mathrm{hr}$ bin average mean peak arrival times. The leading edges for modes $2-4$ are bracketed between the two mode 1 solid lines, and the mean arrivals for modes $2-4$ are positioned above the upper solid line. 


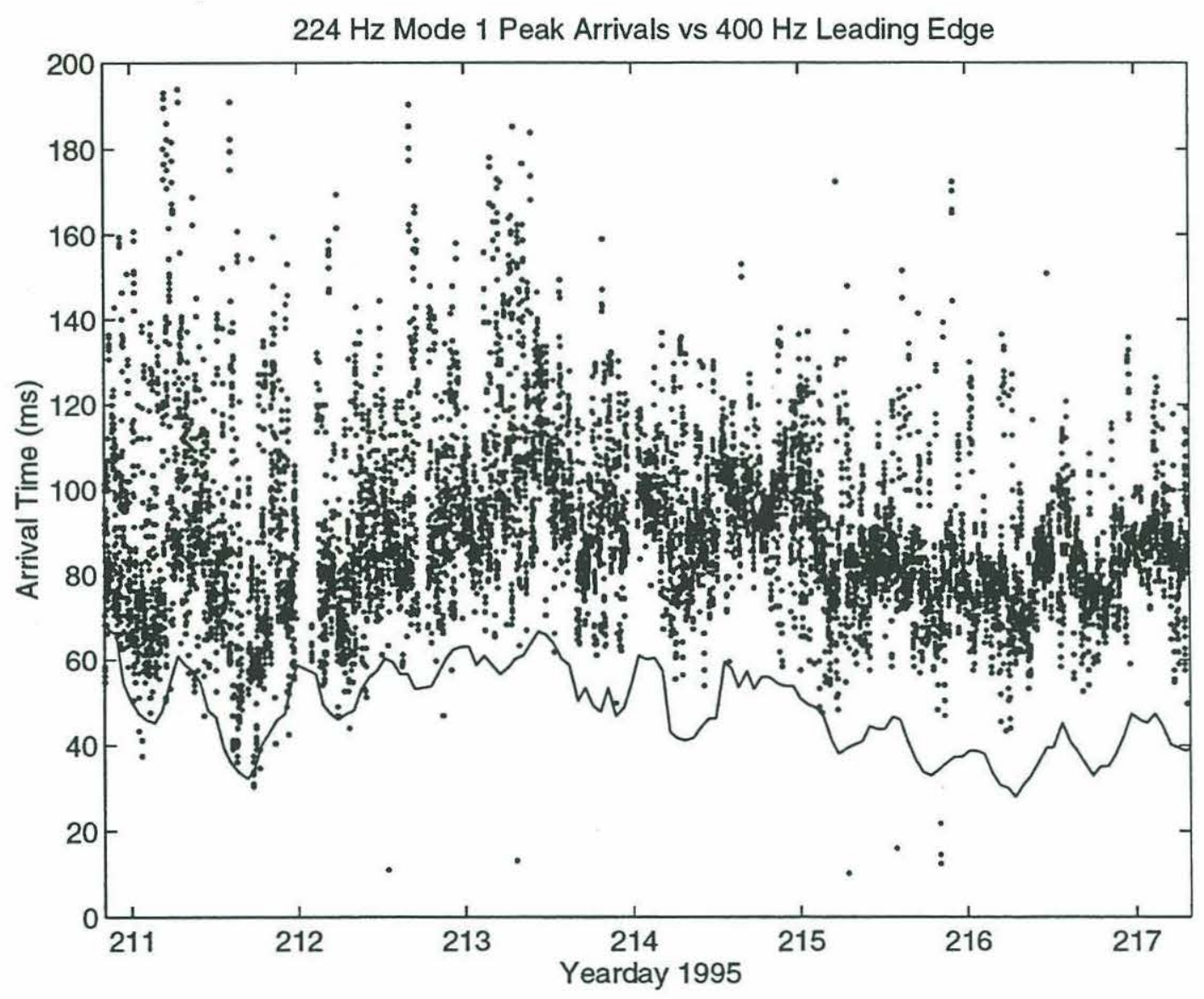

Figure 6-13: Distribution of SWARM $224 \mathrm{~Hz}$ Mode 1 Peak arrival times. The zero reference is at an arrival time of 22.453 seconds. The plotted transmissions occurred every five minutes. Only 8 sequences (every 4 th from 1 to 29) are shown of the 29 available. An upshifted leading edge of the $400 \mathrm{~Hz}$ peak arrival distribution is also shown for comparison. The later zero reference time, relative to Figure 6-1, stems from the difference in source mooring locations. 


\begin{tabular}{|c|c|r|r|r|r|r|r|r|}
\hline \multicolumn{8}{|c|}{$\begin{array}{l}\text { Daily Average } \\
\text { Range Independent Travel Times } \\
\text { in Excess of 21 Seconds (ms) }\end{array}$} \\
\cline { 3 - 9 } Mode & Freq & 211 & 212 & 213 & 214 & 215 & 216 \\
\hline 1 & 224 & 522 & 576 & 586 & 589 & 550 & 483 \\
1 & 400 & 518 & 574 & 579 & 584 & 545 & 476 \\
\hline \multicolumn{2}{|c|}{ Difference } & 4 & 2 & 7 & 5 & 5 & 7 \\
\hline 2 & 224 & 553 & 624 & 632 & 625 & 593 & 539 \\
2 & 400 & 533 & 584 & 603 & 608 & 562 & 504 \\
\hline \multicolumn{2}{|c|}{ Difference } & 20 & 40 & 29 & 17 & 29 & 35 \\
\hline 3 & 224 & 596 & 677 & 668 & 672 & 637 & 597 \\
3 & 400 & 556 & 636 & 640 & 622 & 601 & 542 \\
\hline \multicolumn{2}{|c|}{ Difference } & 40 & 41 & 28 & 50 & 36 & 55 \\
\hline
\end{tabular}

Table 6.3: Hypothetical range independent travel times corresponding to daily averaged SSPs at the WVLA. The travel time differences between $224 \mathrm{~Hz}$ and $400 \mathrm{~Hz}$ modes are also listed.

difficult to estimate just what portion of the variability in the $224 \mathrm{~Hz}$ peak arrival time should be attributed to the broader pulse, but using the shape of the $400 \mathrm{~Hz}$ leading edge as a guide, an uncertainty of around $\pm 5 \mathrm{~ms}$ appears to be appropriate. A similar qualitative analysis of Figure 6-2 leads us to believe the uncertaintity for the $400 \mathrm{~Hz}$ peak arrivals is less than about $\pm 2 \mathrm{~ms}$.

Even with the added uncertainty, tidal modulation in the $224 \mathrm{~Hz}$ PAM1 arrival is still apparent, and the separation between the $224 \mathrm{~Hz}$ and $400 \mathrm{~Hz}$ PAM1 arrival times can be seen to increase by 10 to $15 \mathrm{~ms}$ over the course of the week. One possible source of this divergence is perhaps the frequency dependence of the temporal changes in modal group speed. In support of that hypothesis, Table 6-3 lists hypothetical range independent travel times corresponding to daily averaged SSPs at the WVLA. Unfortunately, the table shows that, at least in the vicinity of the WVLA, temporal changes in mode 1 group speed differences do not appear to be large enough to account for the divergence seen in Figure 6-13. The trend is basically correct, but the range 
in values is only $5 \mathrm{~ms}$. There may be greater (unmeasured) temporal fluctuations at other locations along the acoustic path that might account for the divergence, but an equally likely explanation might involve a fading of the PAM1 arrival as the dominant source of the leading edge. The edge is quite fuzzy to start with, and the loss of a strong PAM1 signal is not unprecedented; PAM1 signal fading is clearly evident in Figure 5-15. A gradual fading of $224 \mathrm{~Hz}$ PAM1 dominance would account for a trend towards increased separation between the $400 \mathrm{~Hz}$ leading edge and the $224 \mathrm{~Hz}$ leading edge.

If one assumes the $224 \mathrm{~Hz}$ leading edge as never being exclusively controlled by the PAM1 arrival time, but rather being influenced by the PAM1, PAM2, and PAM3 arrival times in combination, another explanation of the Figure 6-13 divergence emerges. The PAM2 and PAM3 arrival times have more frequency dependence $(224 \mathrm{~Hz}$ vs. $400 \mathrm{~Hz}$ ) than the PAM1 arrival time, and an average of the three at $224 \mathrm{~Hz}$ produces the sizeable ramp shown in Figure 6-14 (when the $400 \mathrm{~Hz}$ PAM1 arrival time is subtracted). This ramp has the correct trend and shows an average increase in separation of about $15 \mathrm{~ms}$ over six days; but, be cautioned, it does not represent any sort of physical model; it is simply a reasonable combination of statistics with proper physics in the problem that can perhaps support the divergence observed in Figure 6-13.

The rough tidal signal observed in the $224 \mathrm{~Hz}$ arrivals of Figure 6-13 is not inconsistent with the idea of combined influence from all three modes at the leading edge. The leading edges of the higher mode $400 \mathrm{~Hz}$ arrivals (see Figure 6-12) are certainly influenced by the higher mode pseudo-adiabatic arrivals, yet the tidal signal is still evident; the same holds for the mean $400 \mathrm{~Hz}$ arrival times of Figure 6-12.

The corresponding tidal signal fades more rapidly in the $224 \mathrm{~Hz}$ arrivals. Only very rough tidal signals are evident in any of the time series of average $224 \mathrm{~Hz}$ peak arrival times and mean peak heights plotted in Figure 6-15, but as the corresponding power spectral densities (Figure 6-16) illustrate, M2 tidal influences are still present 


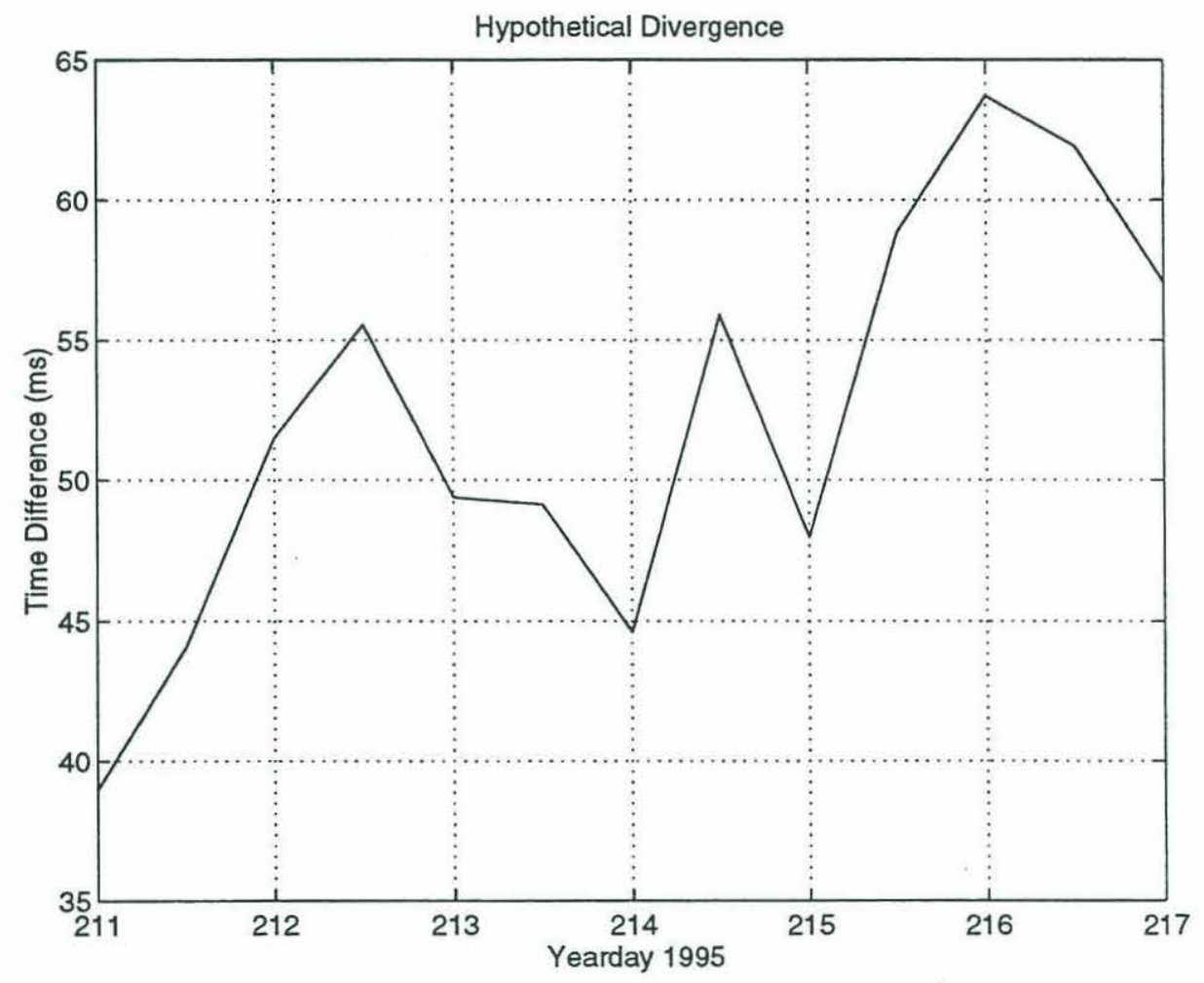

Figure 6-14: Average arrival time difference between the $224 \mathrm{~Hz}$ PAM1, PAM2, and PAM3 arrivals and the $400 \mathrm{~Hz}$ PAM1 arrival. The arrival times are based on 12 hour average SSPs at the WVLA employed in a range independent fashion over a distance of $32 \mathrm{~km}$. 

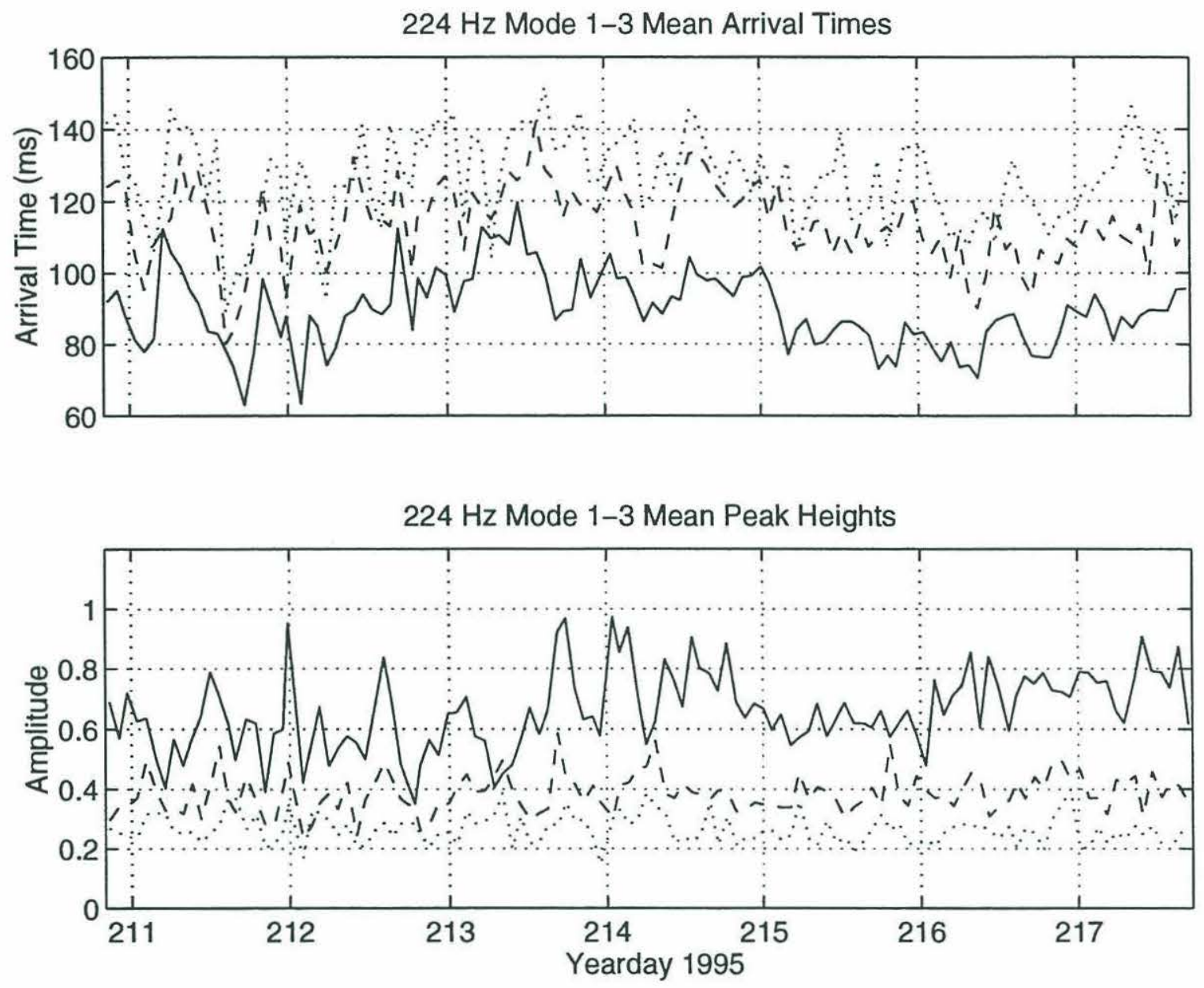

Figure 6-15: $224 \mathrm{~Hz} 1.4 \mathrm{hr}$ bin averaged peak arrival times and peak heights. Mode 1 is solid, mode 2 is dashed, and mode 3 is dotted.

in all six curves. Subtidal oscillations, on the other hand, which are evident in the Figure 6-12 time series of $400 \mathrm{~Hz}$ mean peak arrival time, can still be clearly observed in the time series of the $224 \mathrm{~Hz}$ records.

\subsubsection{Spread Statistics}

The time histories for $224 \mathrm{~Hz}$ and $400 \mathrm{~Hz}$ bin average mode 1 spread (IQR) statistics are compared in Figure 6-17. Both records exhibit fairly strong tidal modulation of spread during the first part of the recorded period. The latter portions of both records have limited tidal signatures, but the modulation that does exist is somewhat 

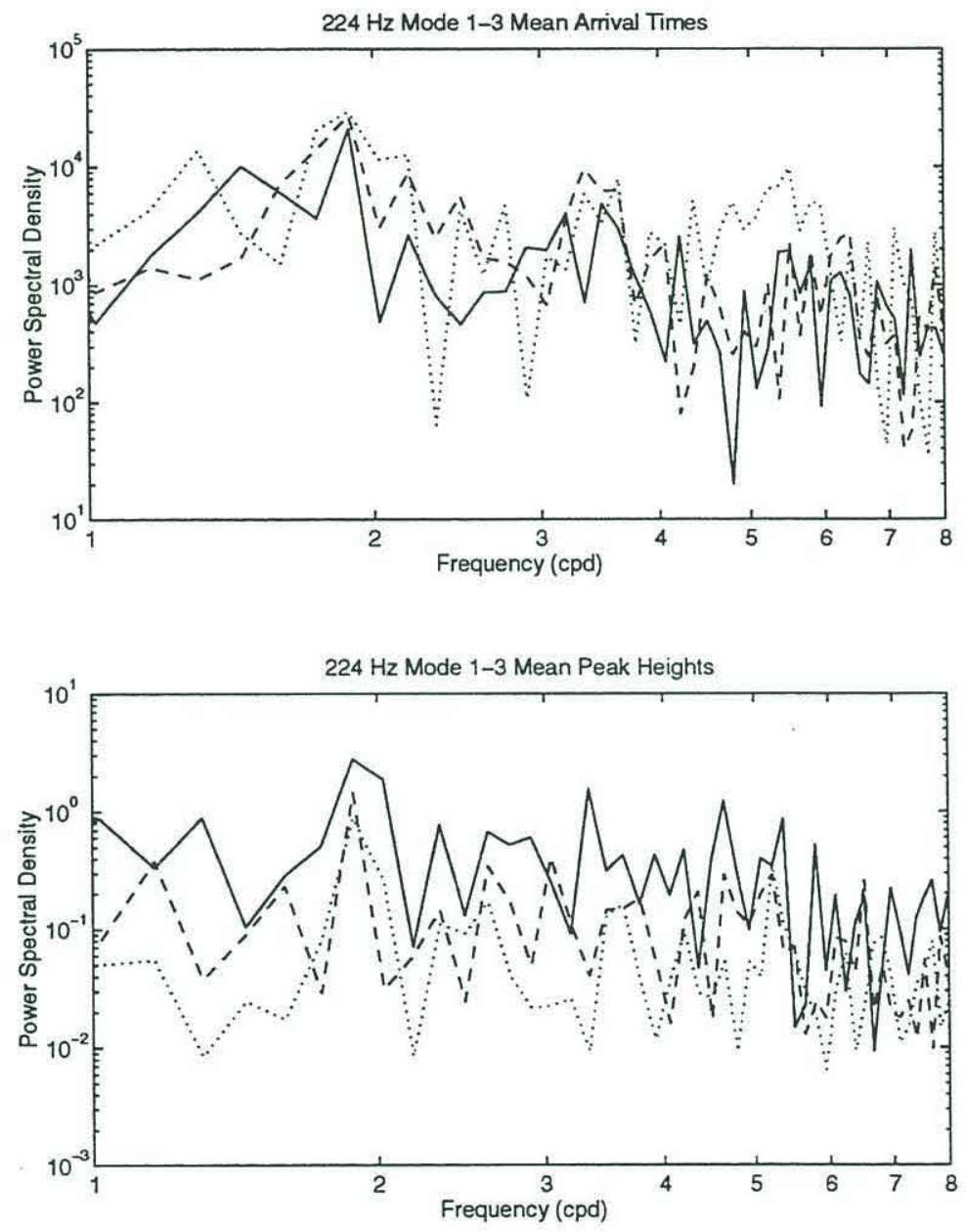

Figure 6-16: Power spectral densities for the $224 \mathrm{~Hz} 1.4 \mathrm{hr}$ bin averaged peak arrival time and peak height time series of Figure 6-15. Mode 1 is solid, mode 2 is dashed, and mode 3 is dotted. 


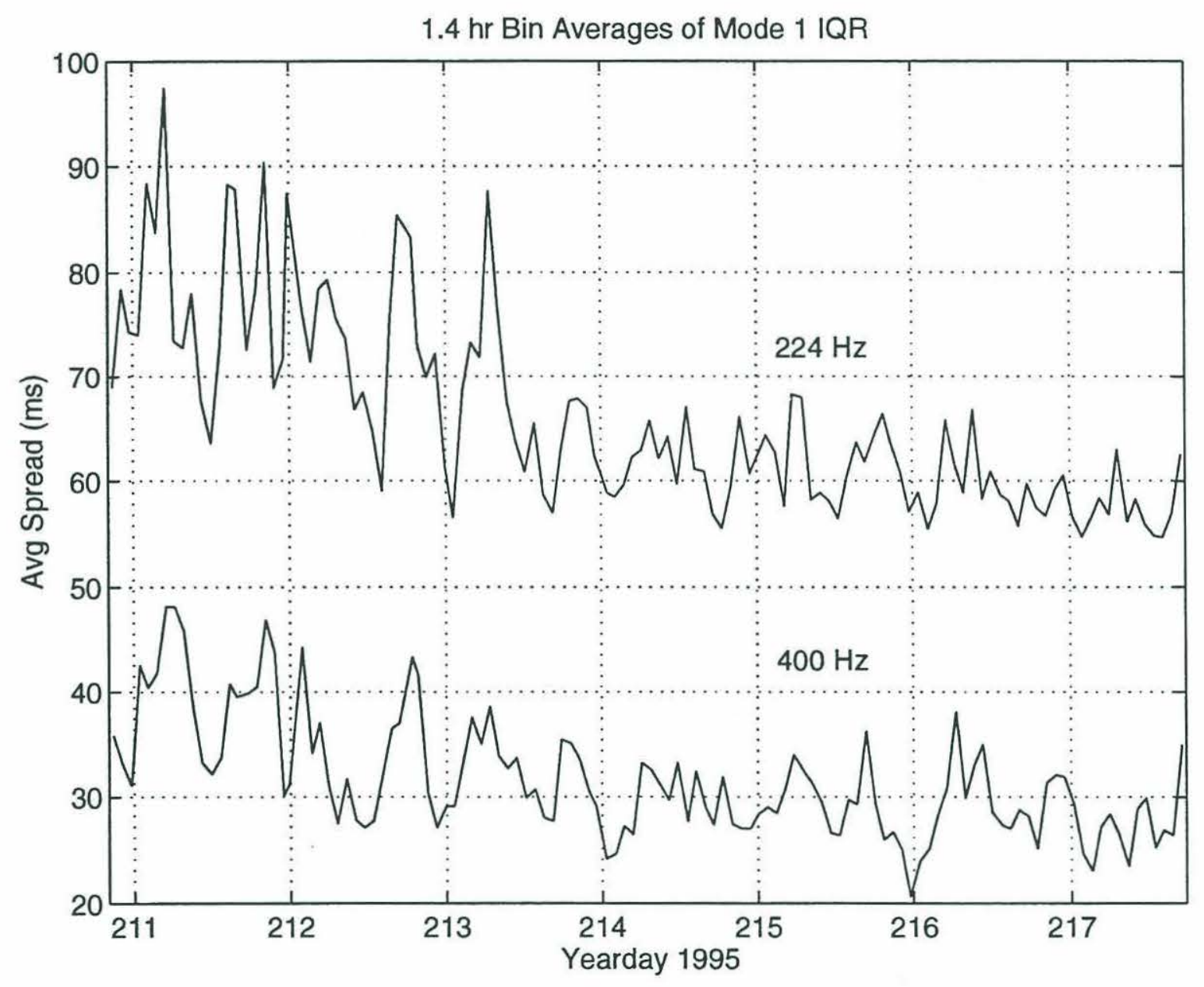

Figure 6-17: Comparison of $224 \mathrm{~Hz}$ and $400 \mathrm{~Hz} 1.4 \mathrm{hr}$ bin averaged IQR (spread). The IQR of an unscattered $224 \mathrm{~Hz}$ pulse would be about $37 \mathrm{~ms}(7 \mathrm{~ms}$ for a $400 \mathrm{~Hz}$ pulse). 
similar, and the mean levels of spread exhibited are both about $23 \mathrm{~ms}$ above their respective unscattered levels of 7 and $37 \mathrm{~ms}$. The zero-lag cross correlation between the two entire records is 0.72 .

\subsubsection{Utility of Further $224 \mathrm{~Hz}$ Analysis}

The analysis of $224 \mathrm{~Hz}$ arrivals has to this point been somewhat limited compared to the analysis of the $400 \mathrm{~Hz}$ arrivals, but, given the reduced resolution of the 224

$\mathrm{Hz}$ signals and the similarity they have exhibited with corresponding $400 \mathrm{~Hz}$ signals, further investigation in this area is less enticing. This is not to say that good information, possibly of better quality than exists at present, cannot be extracted from the $224 \mathrm{~Hz}$ arrivals. Rather, the information obtained would be, for the most part, very similar, but of a poorer quality, to that obtained from the $400 \mathrm{~Hz}$ signals.

Figure 4-7 illustrates the variation in mode shapes from 445 to $355 \mathrm{~Hz}(90 \mathrm{~Hz}$ spread). The differences between 400 and $224 \mathrm{~Hz}$, a $176 \mathrm{~Hz}$ spread, are similar, but as one might expect, larger by a factor of about two. These differences are notable but not dramatic, so there is little to be gained in the way of sampling a different portion of the water column. The $224 \mathrm{~Hz}$ and $400 \mathrm{~Hz}$ coupling characteristics are predictably similar, as evidenced by Figure 6-17, so if additional effort is to be expended on data analysis, the majority should be spent on additional analysis of the clearer $400 \mathrm{~Hz}$ signals. 


\section{Chapter 7}

\section{Conclusions}

\subsection{Conclusions}

The conclusions reached in this investigation, with some overlap, can be placed in two broad categories: modeling and data analysis. The overlap was particularly important in estimating the level of bottom attenuation and verifying the importance of soliton location to the level of signal distortion. A detailed listing of conclusions follows.

\subsubsection{Modeling}

The following conclusions can be drawn from the modeling efforts of Chapter 4 and Chapter 5:

1. The average $400 \mathrm{~Hz}$ compressional wave attenuation in the bottom, along the SWARM acoustic path, is in the neighborhood of 0.02 to $0.03 \mathrm{~dB} / \mathrm{m}$. A sedimentary compressional wave attenuation level of $0.092 \mathrm{~dB} / \mathrm{m}$ can be inferred from chirp sonar inversions for sound speed (linear increase from 1500 to 1800 $\mathrm{m} / \mathrm{s}$ over $30 \mathrm{~m})$ and density $\left(1.8 \mathrm{~g} / \mathrm{cm}^{3}\right)$ in sediment near the WVLA. Extending these bottom parameters in a range independent fashion towards the source produces almost complete attenuation of energy above mode 2 in simulated transmissions, but the actual data shows significant energy transmission 
up through and including mode 4 . An attenuation level of around $0.0115 \mathrm{~dB} / \mathrm{m}$ proved, through numerous trials, to be appropriate for our modeled $30 \mathrm{~m}$ subbottom; slightly higher levels $(0.02$ to $0.03 \mathrm{~dB} / \mathrm{m})$ would be more appropriate if observed along track reductions in the actual SWARM subbottom thickness were considered.

2. Mode arrival spread and bias statistics can be very sensitive to the specific locations of scattering events, but, in general, the more soliton activity there is in the portion of the waveguide closest to the receiver, the greater the spread and bias will be in the mode arrivals. The SIA model results show this very clearly, with the "propagated thermistor string" and actual SWARM data providing good support.

3. Under most shallow water circumstances (i.e. when mode 1 has the highest group speed), the PAM1 arrival time can estimated from a distribution of peak mode 1 arrival times by finding the leading edge envelope of the distribution.

4. Under most shallow water circumstances (i.e. when significant coupling between modes exists), the PAM2 and higher arrival times are not easily discernible from distributions of peak arrival times.

5. At present, the predictive modeling of mode arrivals from soliton filled oceanic waveguides is limited more by inadequate sampling of the environment in question than it is by the limitations of the physics included in the computer models. (It would be extremely difficult to obtain enough environmental data in a SWARM like area to "ground truth" a model, but temperature strings placed every $5 \mathrm{~km}$ or so would probably be enough to put the ball back in the "physics limited" court.) 


\subsubsection{Data Analysis}

The following conclusions can be drawn from the data analysis covered in Chapter 6:

1. Mode 1 has the highest path-averaged group speed for most, if not all, of the duration of the experiment.

2. Large fluctuations in the path-averaged mode 1 group speed occurred over tidal and subtidal periods during the course of the experiment.

3. A significant percentage of the mode 1 group speed fluctuations can be attributed to warm deep water advections in the latter 5 to $10 \mathrm{~km}$ of the waveguide. These advections, which are probably associated with the foot of a warm (and salty) shelfbreak front, can be seen in deep thermistor records near the receiver.

4. Both comparisons with model simulations and correlations with physical measurements point to solitons in the waveguide as the major source of mode coupling during the SWARM experiment.

5. Soliton-filled waveguides will produce mode arrival distortions with fluctuation time scales ranging from minutes to days.

6. The amount of mode arrival distortion a particular waveguide will produce can only be predicted in a general sense (e.g. solitons near the receiver increase the probability of mode 1 spread). Correlations between simple measures of soliton activity and simple measures of mode arrival distortion are weak, but can be seen, when averaged over one or two hours. 


\subsection{Proposed Directions of Future Investigation}

\subsubsection{Modeling}

In the area of modeling, the following ideas should be explored:

1. Investigate the possibility of incorporating depth dependence in the propagated thermistor string model through linear perturbations on depth independent coupling matrix coefficients and depth independent modal wavenumber functions.

2. Look for ways of increasing computational speed through the reduction of repeated calculations of modal wavenumber functions.

\subsubsection{Data Analysis}

In the area of data analysis:

1. The analysis of the WVLA and NRL VLA records should be coordinated to the greatest extent possible. The goal being to obtain similar sets relevant statistics from each instrument.

2. An attempt to measure temporal variations in the mode arrival decorrelation times should be made. The effort will be hampered by the broken nature of the sampling scheme.

\subsubsection{Experiments}

An attempt should be made to conduct a follow-on study. Some suggestions for that study are as follows:

1. Conduct it in a region where there are fewer soliton crossing patterns than were observed at the SWARM site. The ideal being a location that produces regular soliton packets with a substantial quiescent gap between them. 
2. A linear array of four sources at $15,20,25$, and $30 \mathrm{~km}$ from the receiver should be used to study range dependence effects.

3. Each array, as well as the source, should be instrumented with at least six thermistors. Two other strings, placed at 5 and $10 \mathrm{~km}$ from the source, would round out a good sampling of the waveguide. This would aid both modeling efforts and acoustic/environment correlation analysis.

4. Schedule transmissions with two sequences from each source every minute, with the $30 \mathrm{~km}$ source starting on the minute, the $25 \mathrm{~km}$ at 15 seconds, etc. This would provide one pulse compressed sequence per source per minute for analysis.

\subsection{Contributions}

The significant contributions this investigation has supplied to the field, again with some overlap, can be placed in two broad categories: modeling and data analysis.

\subsubsection{Modeling}

The following contributions are drawn from the modeling efforts of Chapter 4 and Chapter 5:

1. A simple method, termed the pulse propagation model (PPM), by which range dependent broadband acoustic propagation can be modeled is developed and employed to evaluate the effects of solitons on acoustic propagation. This PPM method, as shown in Appendix B, compares favorably to synthesizing broadband pulses from KRAKEN [12] complex pressure fields, but it is orders of magnitude faster.

2. By comparison with Marshall Orr's backscatter record, the use of a "propagated thermistor string" record to simulate soliton filled waveguides is shown to be a reasonable first order approach. 
3. The importance of soliton location to a waveguides mode scattering potential is clearly demonstrated.

\subsubsection{Data Analysis}

The following contributions are drawn from the data analysis covered in Chapter 6:

1. The first detailed analysis of actual acoustic propagation in a soliton filled waveguide is presented.

2. Experimental evidence supporting the concept of soliton location being critical to a waveguides mode scattering potential is presented.

3. Experimental evidence supporting the veracity of a "propagated thermistor string" record to simulate soliton filled waveguides is presented. 


\section{Appendix A}

\section{Array Navigation}

\section{A.1 Navigation Set-Up}

Three transponder balls $(11.0,11.5$, and $12.0 \mathrm{kHz})$ were placed in a pattern around the WVLA (Figure A-1). The pattern is not optimum, as the deflections we are concerned with (in the direction of the acoustic path) are nearly orthogonal to both the 11.0 and 11.5 transponder paths. A $10.5 \mathrm{kHz}$ pinger at the base of the array transmitted every four minutes and triggered the transponders. The arrival time of each frequency at the top hydrophone of the WVLA (channel zero) was recorded. The ranges from each transponder to the WVLA were varied to ensure good time separation in the arrivals. With the mean and ramping removed from the traveltimes, any remaining fluctuations that were not common to all three paths would, in theory, be due to motion of the the receiving hydrophone.

\section{A.2 Expected Motions of the Array}

The main mooring consists of a one meter diameter flotation sphere supporting a one inch diameter combination strength member and 16 channel hydrophone array. (Figure A-2). The length of the of the mooring is approximately 60 meters and it 


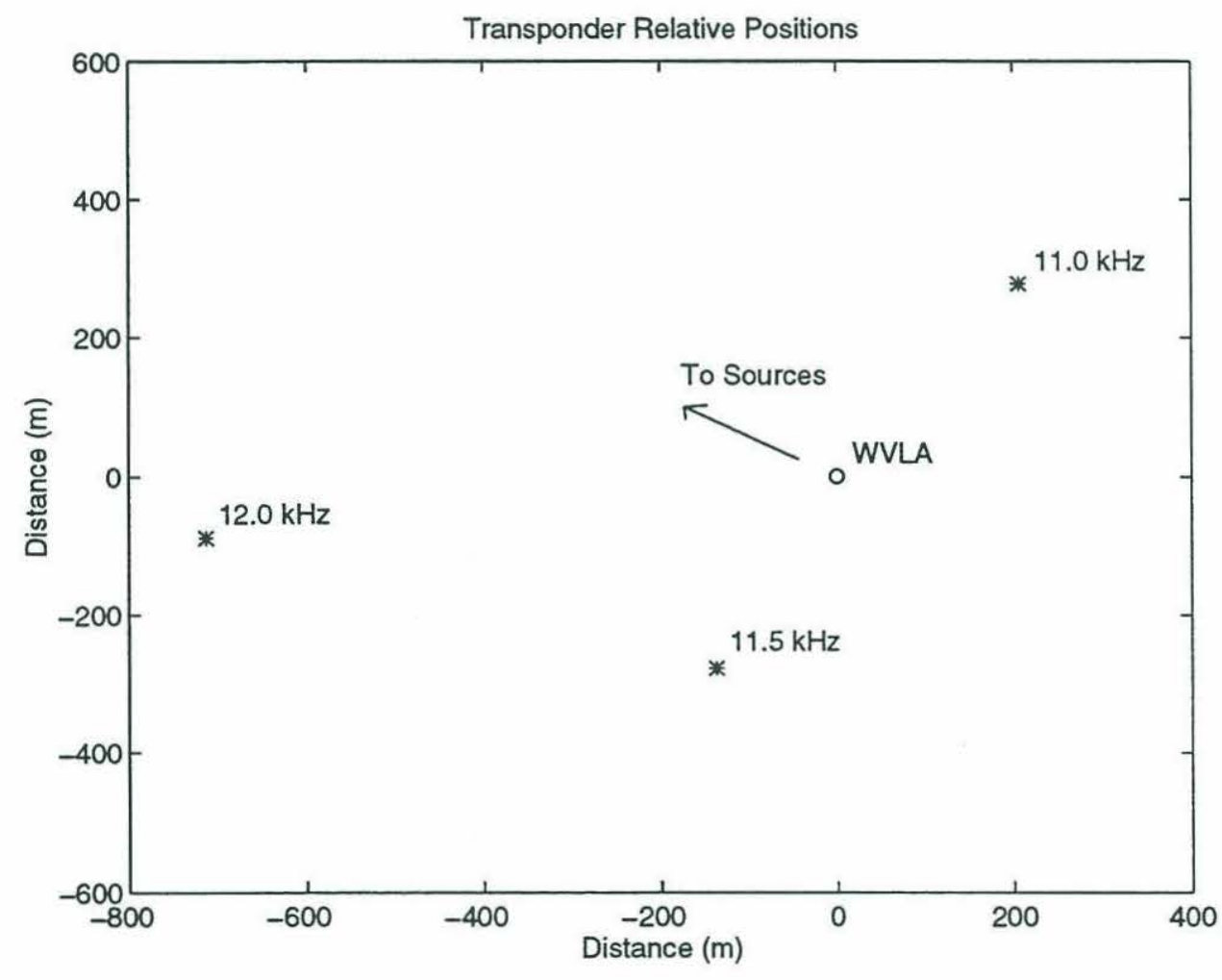

Figure A-1: Relative positions of transponder balls around the WHOI Vertical Line Array (WVLA). Transponders were interrogated every four minutes by a pinger at the base of the array. Round trip travel-time to the top hydrophone of the array was recorded. 


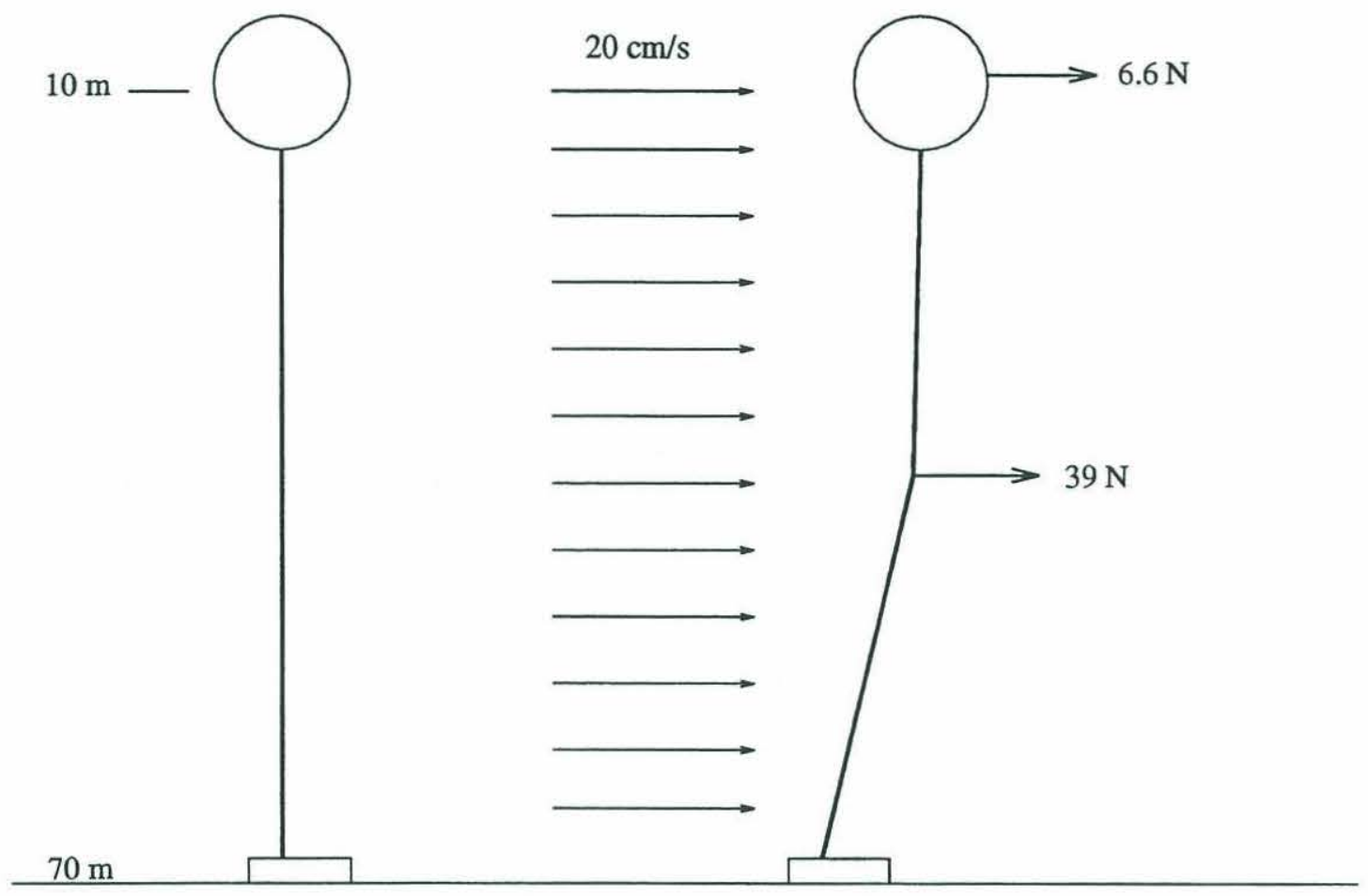

Figure A-2: A simple form drag model for deflection of the WVLA where the drag force on the cable is all applied at the midpoint. The $20 \mathrm{~cm} / \mathrm{s}$ current shown would create a displacement of about $.5 \mathrm{~m}$ at the top hydrophone.

has net a buoyancy of 3230 Newtons. The sum of the added mass and actual mass of the mooring is approximately equal to its displacement, so the undamped natural frequency, $\omega=(g / l)^{\frac{1}{2}}$, is about 3 or 4 cycles per minute. However, large impulseresponse type oscillations are not expected, because the damping from drag is quite significant. It is proportional the square of the flow velocity, with the equivalent linear damping reaching criticality (becoming over-damped) at flows above about $1 \mathrm{~cm} / \mathrm{s}$.

After calculating the form drag of the mooring sphere and cable in a barotropic current field, the tilt of the WVLA can be estimated by determining the equilibrium point between the drag forces and the righting moment provided by the spheres 
buoyancy. At flows around $20 \mathrm{~cm} / \mathrm{s}$ the drag on the sphere can be approximated by,

$$
D_{\text {sphere }} \approx .4\left(\frac{1}{2} \rho v^{2}\right)\left(\pi r^{2}\right)=6.6 N,
$$

and the drag on the cable would be about,

$$
D_{\text {cable }} \approx 1.2\left(\frac{1}{2} \rho v^{2}\right)(.0254 m)(60 m)=39 N \text {. }
$$

If all the cable drag was applied at the midpoint of the cable, the deflection at that point would be,

$$
\Delta x=(30 m) \sin (\arctan (39 / 3230))=.36 m,
$$

and the drag on the sphere would provide an additional,

$$
\Delta x=(60 m) \sin (\arctan (6.6 / 3230))=.12 m,
$$

for a total of about .5 meters at the top hydrophone, which is about $57 \mathrm{~m}$ above the bottom.

A more complicated inelastic cantenary equation solution can be derived by following Triantafyllou [20], replacing his vertical force "cable weight per unit length in water" with our horizontal force "drag per unit length" (our cable is roughly neutrally buoyant). The displacement equation is

$$
x=\frac{V}{D r}\left\{\sqrt{1+\left[\frac{H+D r L}{V}\right]^{2}}-\sqrt{1+\left[\frac{H+D r(L-s)}{V}\right]^{2}}\right\},
$$

where $s$ is the distance along the cable of length $L$ measured from the bottom. When 
the previously computed $20 \mathrm{~cm} / \mathrm{s}$ force quantities,

$$
\begin{aligned}
& V(\text { sphere vertical force })=3230 \mathrm{~N} \\
& H(\text { sphere horizontal force })=6.6 \mathrm{~N} \\
& D r(\text { cable drag })=.65 \mathrm{~N} / \mathrm{m},
\end{aligned}
$$

are inserted, a deflection of $.48 \mathrm{~m}$ is again obtained for the $60 \mathrm{~m}$ point on the cable.

Assuming continuous laminar flow (the sphere probably transitions to turbulence at around $30 \mathrm{~cm} / \mathrm{s}$ ), the displacement for barotropic flows will be roughly proportional to the square of the current speed, giving 1 meter at $30 \mathrm{~cm} / \mathrm{s} ; 2$ meters at $40 \mathrm{~cm} / \mathrm{s}$; and 3 meters at $50 \mathrm{~cm} / \mathrm{s}$.

Figure A-3 shows the upper and lower depth bins of a bottom-mounted acoustic Doppler current meter (ADCP) moored about $3 \mathrm{~km}$ from the WVLA. Most of the barotropic currents are less than $30 \mathrm{~cm} / \mathrm{s}$. The largest currents are baroclinic soliton currents which have a westward component near the surface and an eastward component near the bottom. The effects of baroclinic currents on mooring motion are hard to predict, but the movement at the top hydrophone is certainly less than what would be produced by a barotropic current of the same magnitude. This line of thought coupled with the ADCP data predicts mooring motions that are generally less than a meter in amplitude at the top hydrophone (equivalent to 1 degree of tilt) with occasional excursions that might approach 2 meters.

\section{A.3 Navigation Data Analysis}

\section{A.3.1 Inversion for Mooring Motion}

Initial inspection of the navigation data reveals travel-time fluctuations ranging from one to five milliseconds (Figure A-4). This roughly translates to deflections in hydrophone position of from 1.5 to 7.5 meters. A fairly clear tidal signal is apparent

throughout the $11.0 \mathrm{Khz}$ record; the tidal signal is weaker in the $11.5 \mathrm{Khz}$ signal, 


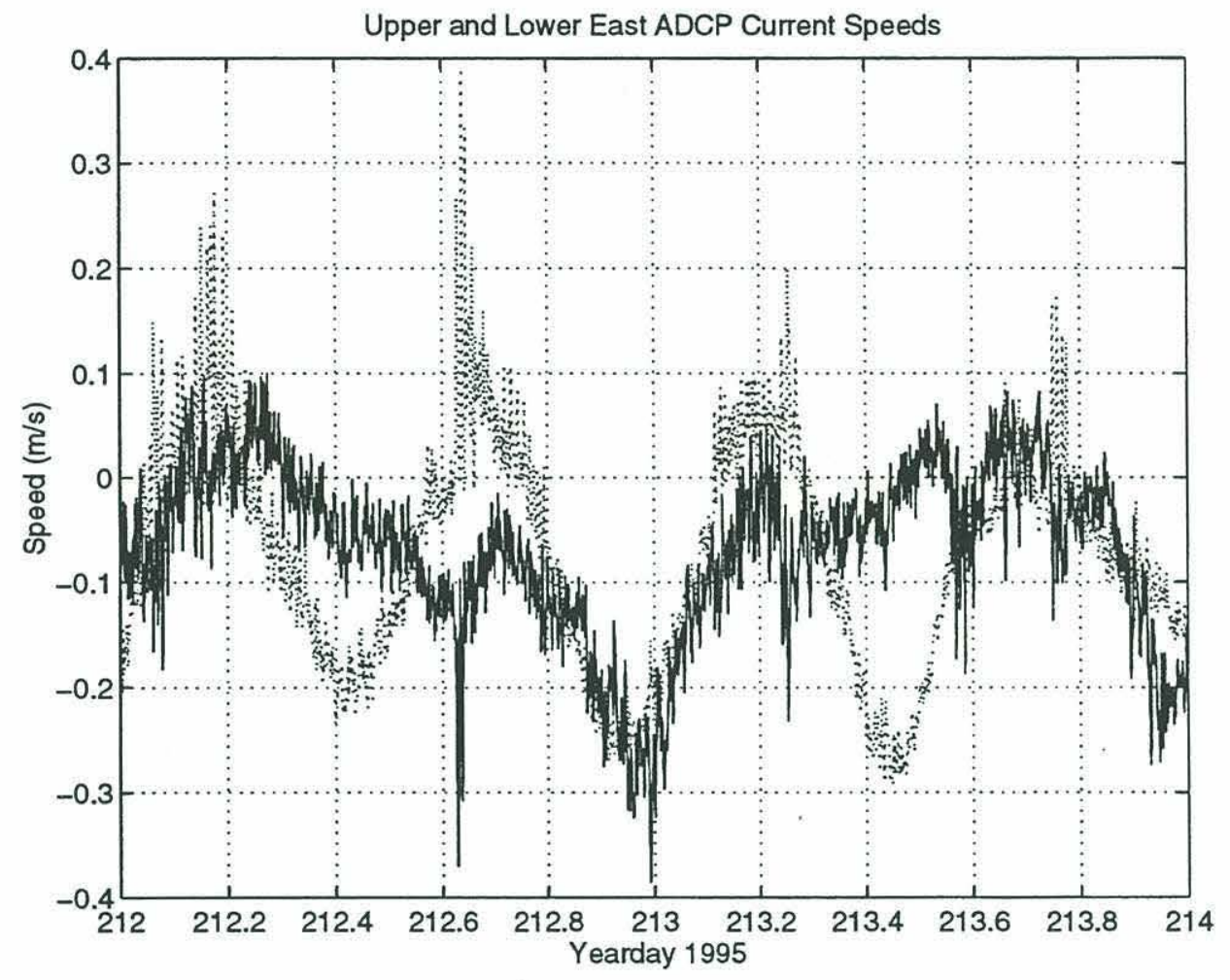

Figure A-3: The first (..) and fourteenth (-) depth bins of a 16 channel bottommounted acoustic Doppler current meter (ADCP) moored about $3 \mathrm{~km}$ from the WVLA. 


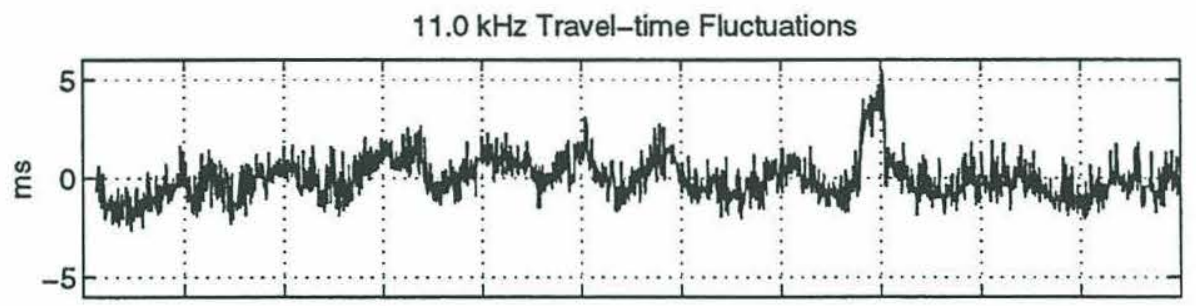

$11.5 \mathrm{kHz}$ Travel-time Fluctuations
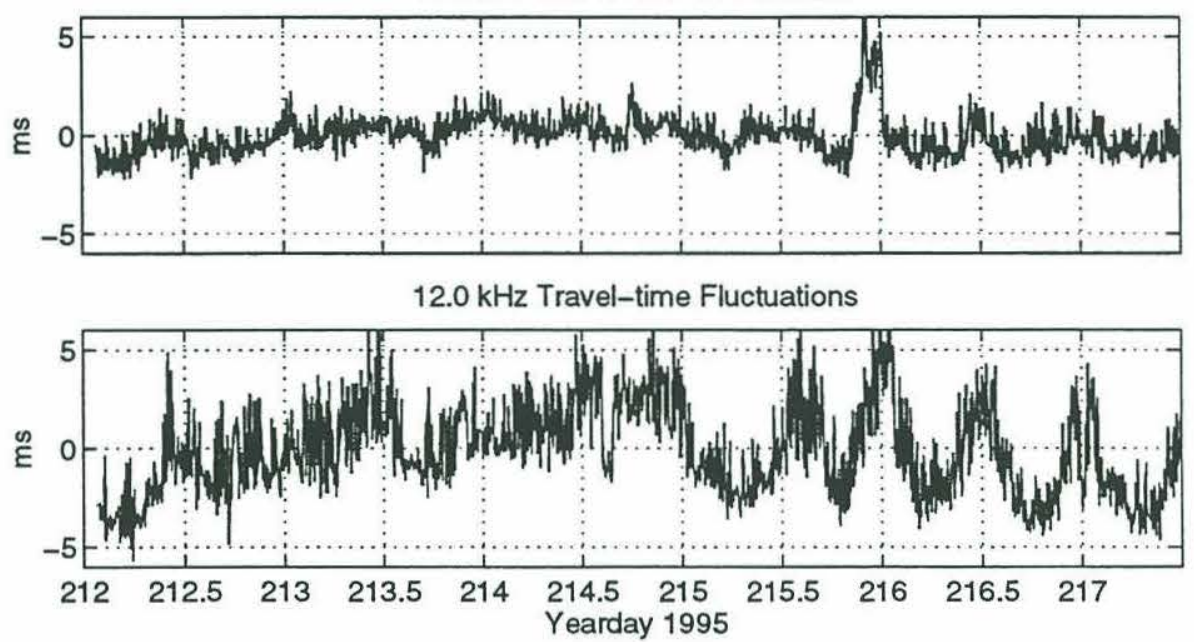

Figure A-4: Travel-time fluctuation data-vectors associated with the three transponder ball paths used to navigate the WVLA.

nonexistent in the first half of the $12.0 \mathrm{Khz}$ record, but very strong in the latter portions of the $12.0 \mathrm{Khz}$ record.

The travel-time fluctuations can be more directly converted to equivalent hydrophone displacement using a least-squares inversion of the following model,

$$
\frac{-1}{c}\left|\begin{array}{cc}
\cos \theta_{1} & \sin \theta_{1} \\
\cos \theta_{2} & \sin \theta_{2} \\
\cos \theta_{3} & \sin \theta_{3}
\end{array}\right|\left|\begin{array}{c}
\Delta x \\
\Delta y
\end{array}\right|=\left|\begin{array}{c}
\Delta t_{1} \\
\Delta t_{2} \\
\Delta t_{3}
\end{array}\right|,
$$



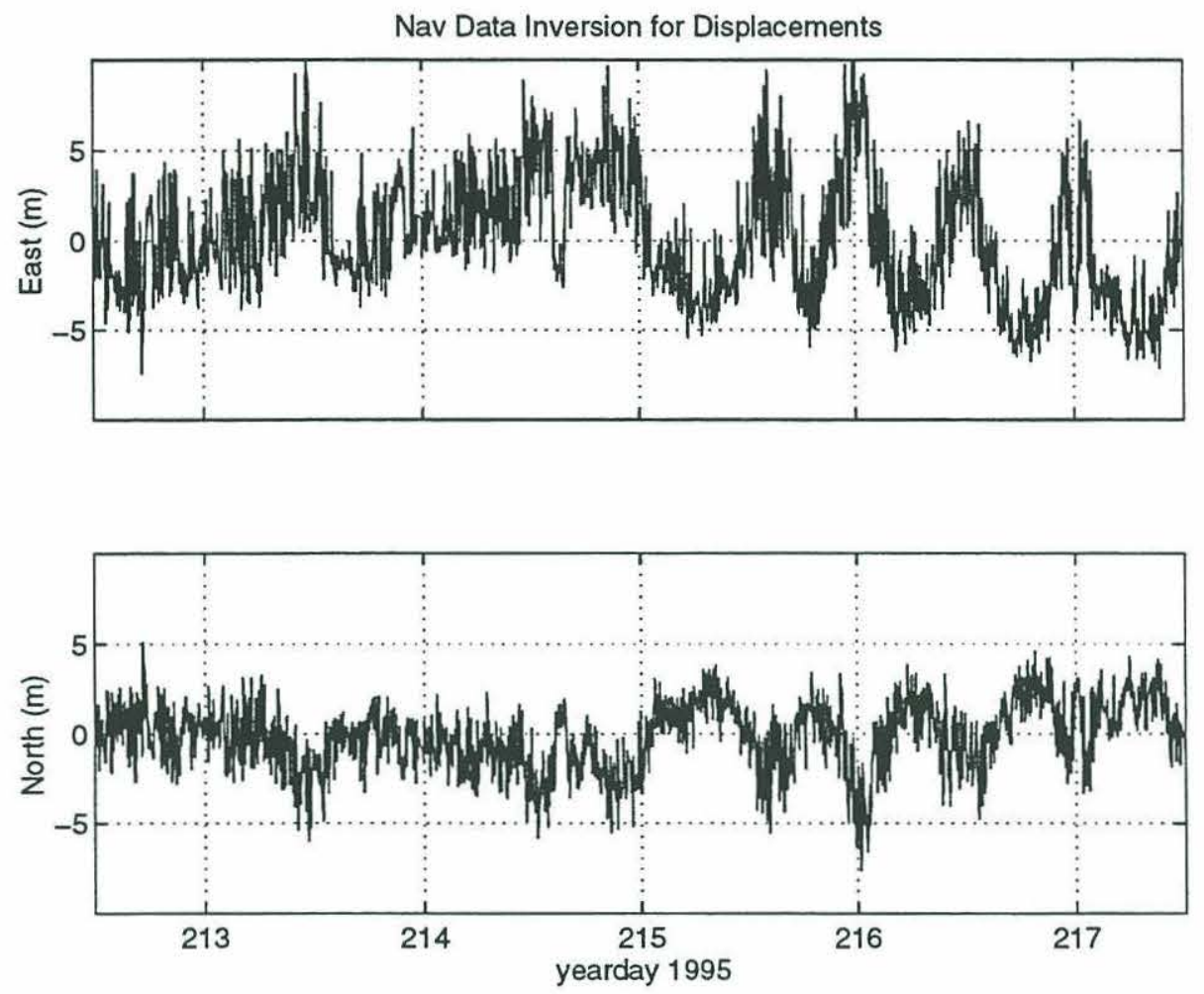

Figure A-5: Model vectors of an over-determined least-squares inversion of the navigation data for north and east displacement of the hydrophone array.

where,

$$
\begin{aligned}
& \theta_{n}=\text { relative bearing of due east from transponder bearing, } \\
& \Delta x=\text { east displacement } \\
& \Delta y=\text { north displacement } \\
& \Delta t_{n}=\text { travel-time fluctuation at transponder }
\end{aligned}
$$

and

$$
c=1500 \mathrm{~m} / \mathrm{s} \text {. }
$$

Figure A-5 illustrates two problems with the inverted displacements: they are much larger than expected from the ADCP data and form drag calculations; and the source of the high frequency oscillations is unclear.

The low frequency oscillations in the latter part of the record have a clear tidal signature, the east-west component being forced almost directly by the $12.0 \mathrm{Khz}$ 
transponder. The orientation of the tidal ellipse in the area is $319 \operatorname{deg} \mathrm{T}$ for the major axis component ( $\sim 14 \mathrm{~cm} / \mathrm{s})$ and $231 \mathrm{deg} \mathrm{T}$ for the minor axis component ( $~ 9$ $\mathrm{cm} / \mathrm{s}$ ). Given this tidal ellipse and the strong $12.0 \mathrm{Khz}$ tidal signal starting on day 215 , we would expect to see reasonably strong tidal signals from the 11.0 and 11.5 transponders, but we don't.

The source of the high frequency displacement signal is even more troublesome. If we choose to believe the low frequency inverted displacements of order $\pm 5 \mathrm{~m}$ are caused by tidal currents (i.e. gross errors in hydrodynamic calculations), then we must also accept the strong possibility that the higher frequency displacements of order $\pm 2.5 \mathrm{~m}$ are real, the result of high frequency current oscillations (see Figure A-3).

Another source of both high and low frequency transponder travel-time fluctuations is soundspeed changes. An internal tide can depress the thermocline causing a travel-time change that is linearly proportional to the affected pathlength; since the distance to the $12.0 \mathrm{Khz}$ transponder is twice as far as the the 11.0 and 11.5 transponders, our previous inversion could interpret this as a mooring motion. This does not appear to be a significant part of the tidal signal in the $12.0 \mathrm{Khz}$ record. If it were, we would expect to see similar fluctuations at about half the amplitude of 12.0 fluctuations in the 11.0 and 11.5 records.

The level of influence from internal tide displacements can be estimated more directly by using a modified form of the previous inversion (no longer over-determined) that solves for an average sound speed fluctuation along with the east and north hydrophone displacements. The speed deviation in this case is assumed to apply to the entire return path from each transponder to the upper hydrophone of the WVLA. In actuality it only applies to a portion of each path, but for long waves, the affected pathlength will be roughly a common fraction of each total pathlength. The near bottom paths from the interrogator to the transponders are assumed to have a stable 
sound speed. This inversion,

$$
\frac{-1}{c}\left|\begin{array}{ccc}
\cos \theta_{1} & \sin \theta_{1} & d_{1} / c \\
\cos \theta_{2} & \sin \theta_{2} & d_{2} / c \\
\cos \theta_{3} & \sin \theta_{3} & d_{3} / c
\end{array}\right|\left|\begin{array}{c}
\Delta x \\
\Delta y \\
\Delta c
\end{array}\right|=\left|\begin{array}{c}
\Delta t_{1} \\
\Delta t_{2} \\
\Delta t_{3}
\end{array}\right|
$$

where,

$$
\Delta c=\text { path average change in sound-speed, }
$$

and

$$
d_{n}=\text { distance from WVLA to transponder, }
$$

produces smaller amplitudes (still larger than expected) for the low frequency mooring motions (Figure A-6), and the added model vector for speed deviation shows some similar (and, as expected, smaller magnitude) fluctuations when compared to a record of sound speed in the upper water column (Figure A-7). The sound speed record is converted from a temperature record at a depth of 12.5 meters on the WVLA. This inversion shows no diminution in high frequency mooring motion; we would not expect high frequency soundspeed deviations to fit into the long wave assumption.

\section{A.3.2 Inversion with Temperature Correction Applied to Data}

The similarity between the thermistor record and the average sound speed model vector opens the possibility for applying a sound speed correction to the data vectors prior to inversion for mooring motion. As it turns out, the travel-time fluctuation data vectors can be modified slightly by applying the predicted local change in sound speed from the thermistor data to one eighth (a reasonable and optimum fraction for 

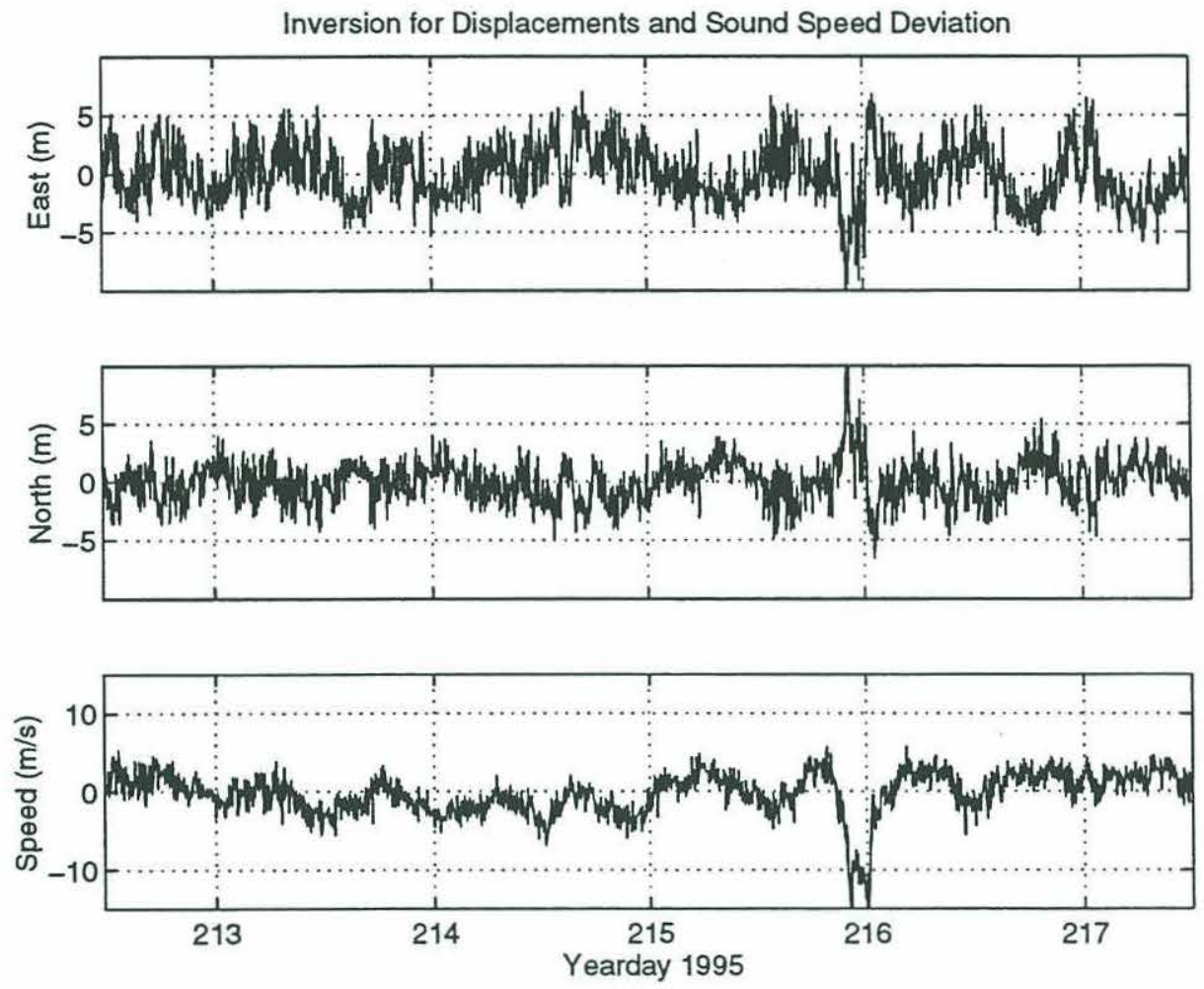

Figure A-6: Model vectors of a least-squares inversion of the navigation data for north displacement, east displacement, and an average deviation in sound speed common to all three transponder return path integrals. 

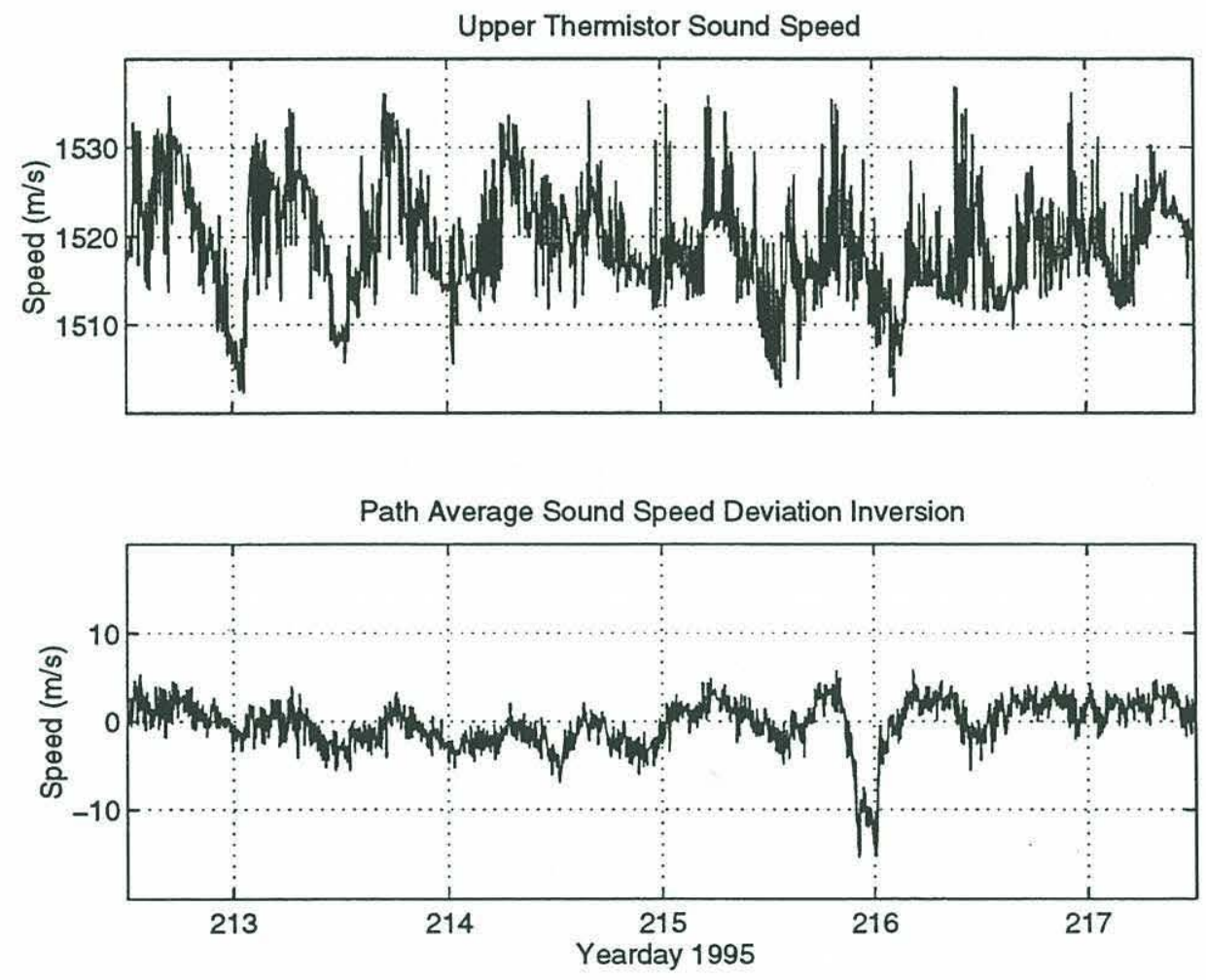

Figure A-7: A least-squares inversion for sound speed deviation produces fluctuations similar those observed in a 12.5 meter depth thermistor record that has been converted to sound speed. 
this data set) of the return path for all three transponders, but this inversion,

$$
\left|\begin{array}{cc}
\cos \theta_{1} & \sin \theta_{1} \\
\cos \theta_{2} & \sin \theta_{2} \\
\cos \theta_{3} & \sin \theta_{3}
\end{array}\right|\left|\begin{array}{c}
\Delta x \\
\Delta y
\end{array}\right|=\left|\begin{array}{c}
c \Delta t_{1}+\frac{d_{1} \Delta c}{8 c} \\
c \Delta t_{2}+\frac{d_{2} \Delta c}{8 c} \\
c \Delta t_{3}+\frac{d_{3} \Delta c}{8 c}
\end{array}\right|,
$$

is not a significant improvement over

$$
\left|\begin{array}{cc}
\cos \theta_{1} & \sin \theta_{1} \\
\cos \theta_{2} & \sin \theta_{2} \\
\cos \theta_{3} & \sin \theta_{3}
\end{array}\right|\left|\begin{array}{c}
\Delta x \\
\Delta y
\end{array}\right|=\left|\begin{array}{c}
c \Delta t_{1} \\
c \Delta t_{2} \\
c \Delta t_{3}
\end{array}\right|
$$

because applying the sound speed corrections to the data vectors only slightly decreases their variances from $2.4,2.3$, and $12.3 \mathrm{~m}^{2}$ to $2.2,2.2$, and $12.0 \mathrm{~m}^{2}$.

Utilizing the thermistor sound speed deviation over larger portions of the return path will actually increase the variance of the data vectors. This is not unexpected; the return paths start out deep $(70 \mathrm{~m})$ where the effects of solitons on the sound speed are not so pronounced as one will find at 12.5 meters. Also, it must be kept in mind that the thermistor record is influenced by both global and local temperature fluctuations.

\section{A.3.3 Inversion for Sound Speed Only}

Internal tides have 20 to $30 \mathrm{~km}$ wavelengths, but internal wave solitons have wavelengths on the order of 200 meters and could be an important source of the high frequency oscillations seen in the transponder records. An over-determined inversion looking only for speed fluctuations can be constructed to solve for two separate sound speed fluctuations. The 11.0 and 11.5 paths are more likely than any other pair to have speed deviations in common. The predominant direction of solitary wave propagation is $300^{\circ}$ by compass heading, so with the soliton phase fronts perpendicular 
to the propagation direction, the 11.0 and $11.5 \mathrm{kHz}$ locations could very possibly experience the simultaneous passage of a solitary wave (Figure A-1). An inversion that solves for a sound speed fluctuation common to the 11.0 and $11.5 \mathrm{kHz}$ transponder return path and a separate fluctuation for the $12.0 \mathrm{kHz}$ return path,

$$
\frac{-1}{c^{2}}\left|\begin{array}{cc}
d_{1} & 0 \\
d_{2} & 0 \\
0 & d_{3}
\end{array}\right|\left|\begin{array}{c}
\Delta c_{12} \\
\Delta c_{3}
\end{array}\right|=\left|\begin{array}{c}
\Delta t_{1} \\
\Delta t_{2} \\
\Delta t_{3}
\end{array}\right|,
$$

produces very interesting results. Figure A-8 shows that the deviations in sound speed from this inversion are again similar to the temperature (converted to speed) changes recorded at the top of the WVLA. A closer comparison between inverted speed deviations for the 11.0 and $11.5 \mathrm{kHz}$ transponders, $\Delta c_{12}$, and a demeaned upper thermistor speed record shows very good agreement for the early part of the record (Figure A-9).

A comparison between the $12.0 \mathrm{khz}$ travel-times and the two-way transponder range divided by the average of the three deepest sound speeds on the WVLA is shown in Figure A-10. While Figure A-9 indicates some sensitivity for shallow temperature fluctuations in the $11.0 / 11.5 \mathrm{khz}$ records, this figure shows the $12.0 \mathrm{khz}$ to be very sensitive to deep temperature fluctuations. The size and path dependence of these temperature signals make any inversion for mooring motion unreliable.

\section{A.4 Conclusions}

The following conclusions are reached in regard to navigation data at the WVLA:

1. The average length scale of temperature fluctuations in the vicinity of the WVLA was less than $1 \mathrm{~km}$ (i.e. most temperature fluctuation signals were not common to all three paths). 

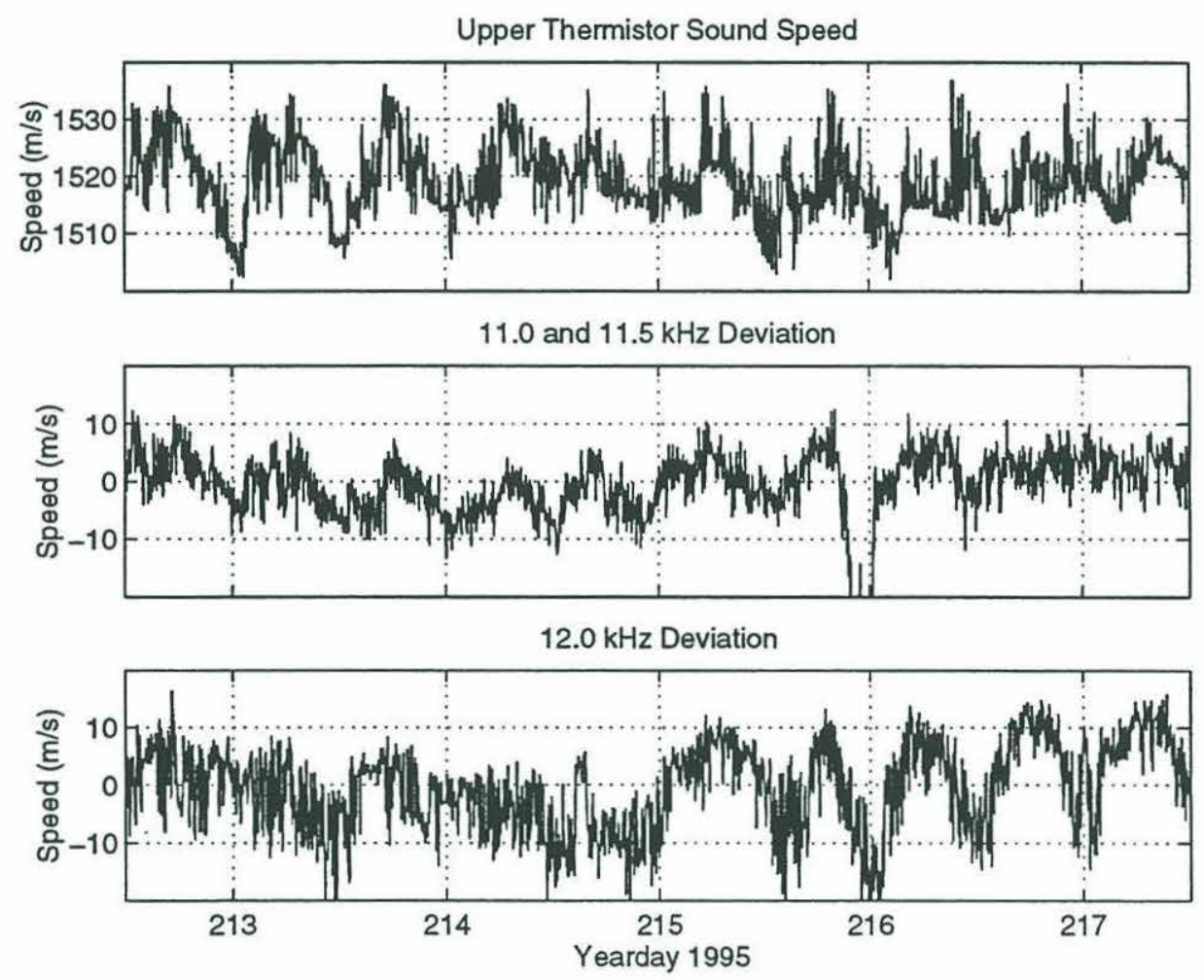

Figure A-8: A least-squares inversion for sound speed deviation produces fluctuations similar those observed in a 12.5 meter depth thermistor record that has been converted to sound speed. 


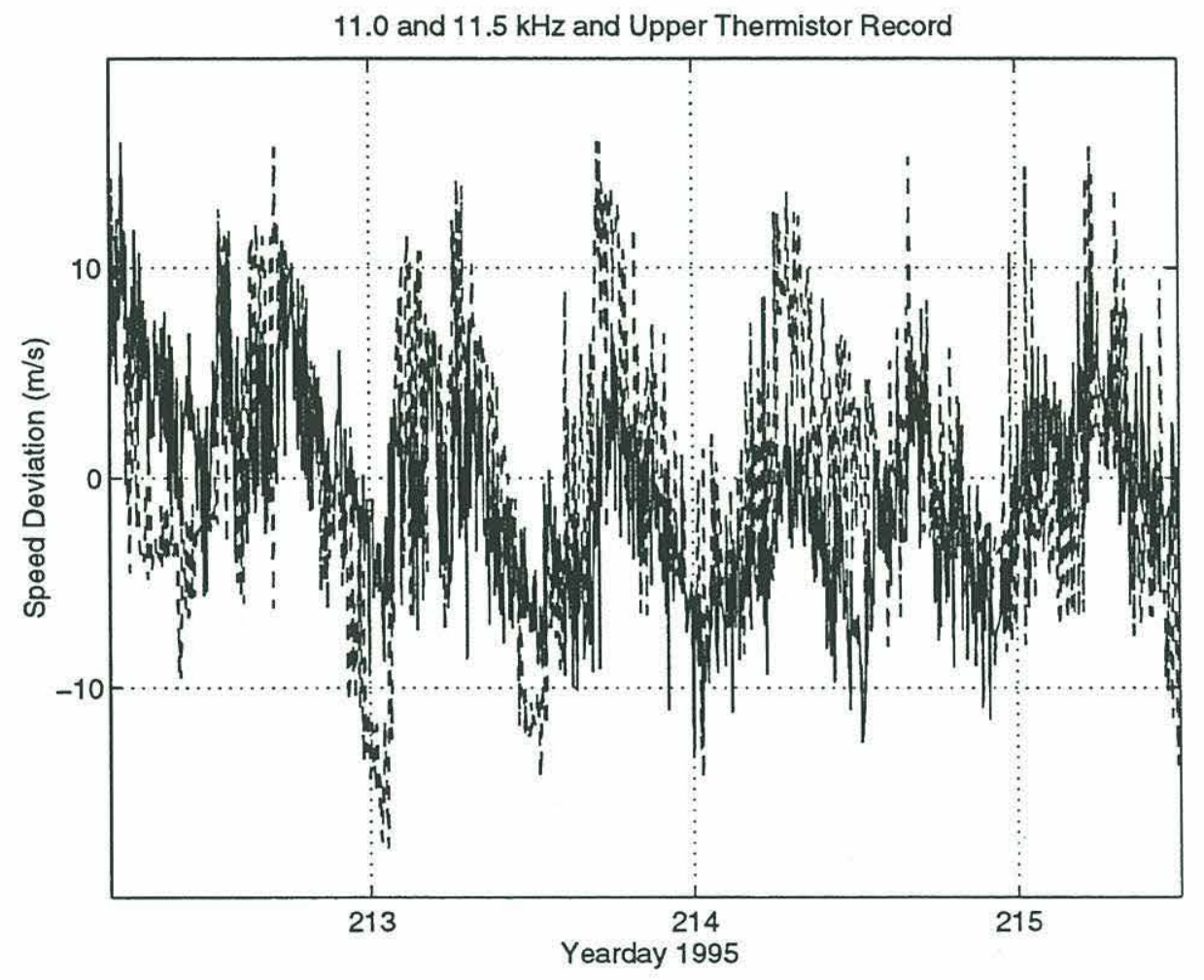

Figure A-9: A least-squares inversion for sound speed deviations yields an 11.0/11.5 $\mathrm{kHz}$ path sound speed fluctuation (solid line) that is very similar those observed in a 12.5 meter depth thermistor record (dashed line). 


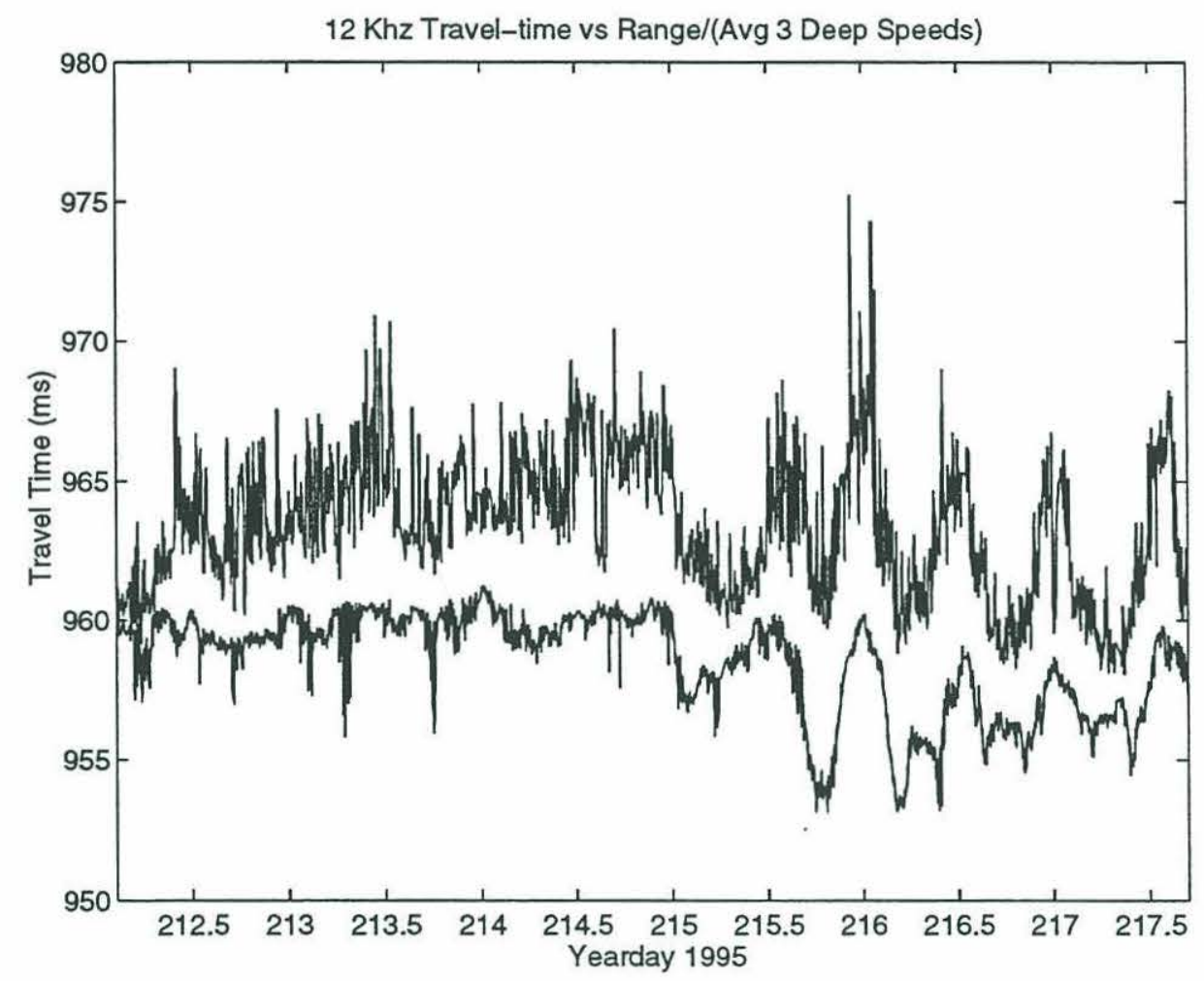

Figure A-10: $12.0 \mathrm{kHz}$ path travel-time fluctuations (upper line) are very similar those calculated by taking the range to the transponder and back divided the average of the WVLA soundspeeds at 40,50, and 60 meters depth. 
2. Mooring motion signals in the navigation data were too small to be detected above the background of temperature fluctuations.

3. Hydrodynamic estimates limit mooring motions to less than one degree of tilt for most periods, with occasional excursions approaching two degrees during the passage of large solitons. 


\section{Appendix B}

\section{Comparison of the Pulse}

\section{Propagation Model with Pulse}

\section{Synthesis using KRAKEN}

\section{Complex Pressure Fields}

The pulse propagation model (PPM) described in Chapter 4 relies on the KRAKEN[12] normal mode code for computation of mode shapes, wave numbers, and attenuation levels. The acoustic FIELD program, within the KRAKEN model, can also compute the complex pressure field, $C(r, z, \omega)=a(r, z, \omega)+i b(r, z, \omega)$, at a number of selected ranges and depths for a given source depth, source frequency, and acoustic environment.

If the field calculations are repeated for a number of frequencies (covering the bandwidth of the transmitted signal), the resulting complex spectrum of the field at a given point, when multiplied by the Fourier components of the source pulse, $P(\omega)$, can, after Fourier synthesis, yield a time domain pulse,

$$
p(r, z, t)=\frac{1}{2 \pi} \int C(r, z, \omega) P(\omega) e^{i \omega t} d \omega .
$$


Since complex pressure fields are not smooth functions of frequency, an accurate solution using the SWARM $400 \mathrm{~Hz}$ source requires 1201 KRAKEN runs for each different SSP in a range dependent problem. This procedure, which we will refer to as KRAKEN Field Synthesis (KFS), computes the arrival at a single hydrophone; it can be extended to predict mode arrivals by mode-filtering the predicted arrivals at vertical array of hydrophones.

Conversely, the mode arrival predictions of the pulse propagation model can be synthesized to yield the arrival at a given single hydrophone. This computation,

$$
p(r, z, t)=\sum_{n} \tilde{p}_{n}(r, t) \phi_{n}\left(z_{s}\right) \phi_{n}(z)
$$

where the $\tilde{p}_{n}(r, t)$ are the mode arrivals, the $\phi(z)$ are the mode shapes, and $z_{s}$ is the source depth, is very simple and provides an output for direct comparison with the KFS method. Since the modal wavenumber spectra it uses for propagation are fairly smooth functions of frequency, the PPM method requires only 5 KRAKEN runs for each SSP. The wavenumbers and attenuation levels for each modes 1201 non-zero frequency bins are then computed through cubic spline interpolation. The 5 KRAKEN runs with interpolation used in the PPM are orders of magnitude more efficient than the 1201 KRAKEN runs needed in KFS.

Figure B-1 shows a comparison between the two methods for simple range dependent case. The modeled waveguide uses the SSPs shown in Figure 4-6, with coupling from one SSP to the other occurring at 13,000 and $18,000 \mathrm{~m}$. The agreement between the two methods is very good at all ranges for this case.

A more complicated scenario (Figure B-2) yields results that are not as closely matched but still quite good, even at the $20 \mathrm{Km}$ point. There are several potential contributors to the deviation between the PPM and KFS results. The most obvious is the narrowband approximation used in PPM coupling matrices. The mode shapes, as illustrated in Figure 4-7, do have a slight dependence on frequency, and as such, the coupling matrices are also frequency dependent. This dependence is accounted 

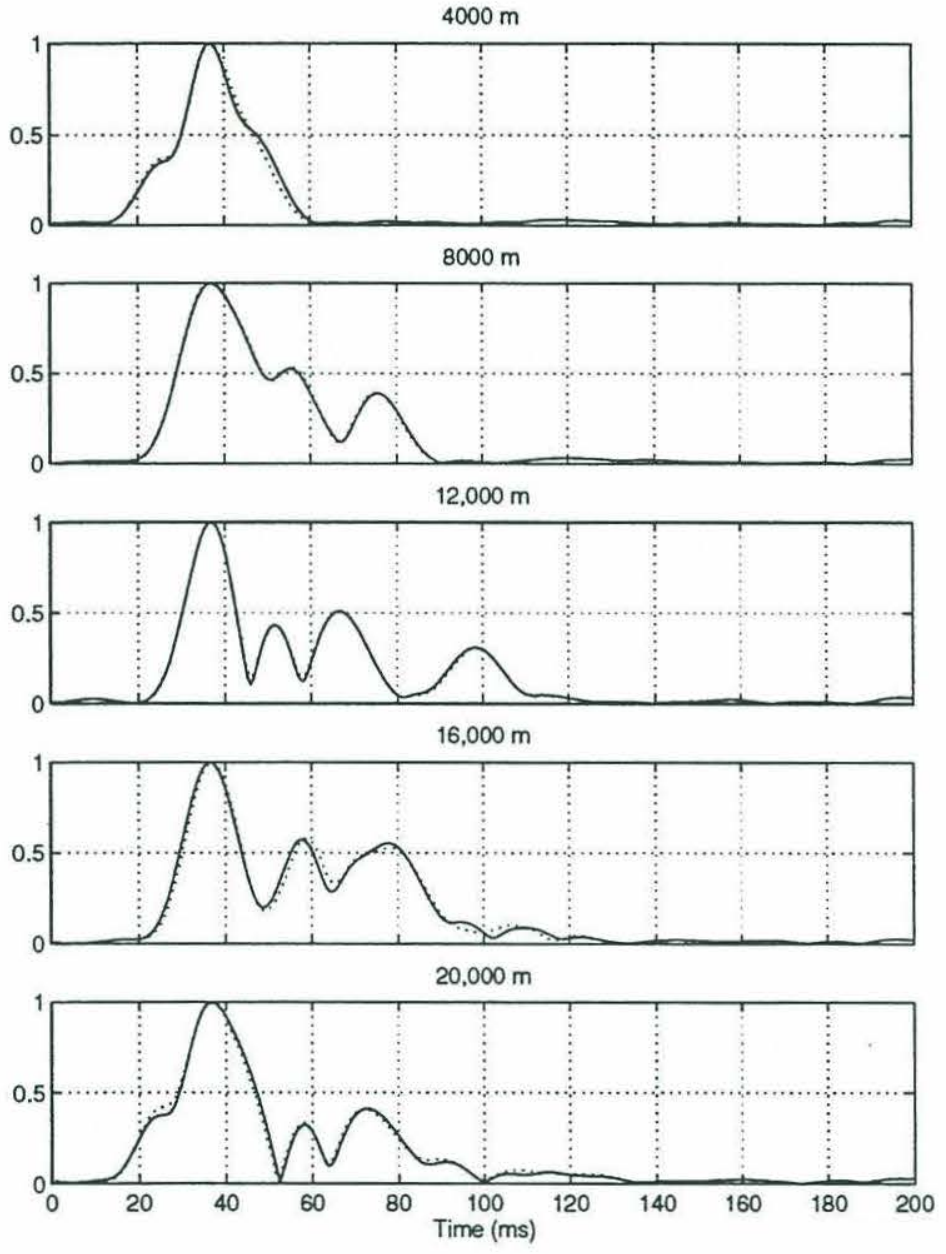

Figure B-1: A comparison of single hydrophone pulse arrivals computed from the PPM (solid) and KFS (dotted) methods. The modeled waveguide uses the SWARM SSPs shown in Figure 4-6, with coupling from one SSP to the other occurring at 13,000 and $18,000 \mathrm{~m}$. The source depth is $27 \mathrm{~m}$ and the receiver depth at all five ranges is $50 \mathrm{~m}$. 
Propagation through SIA Solitons
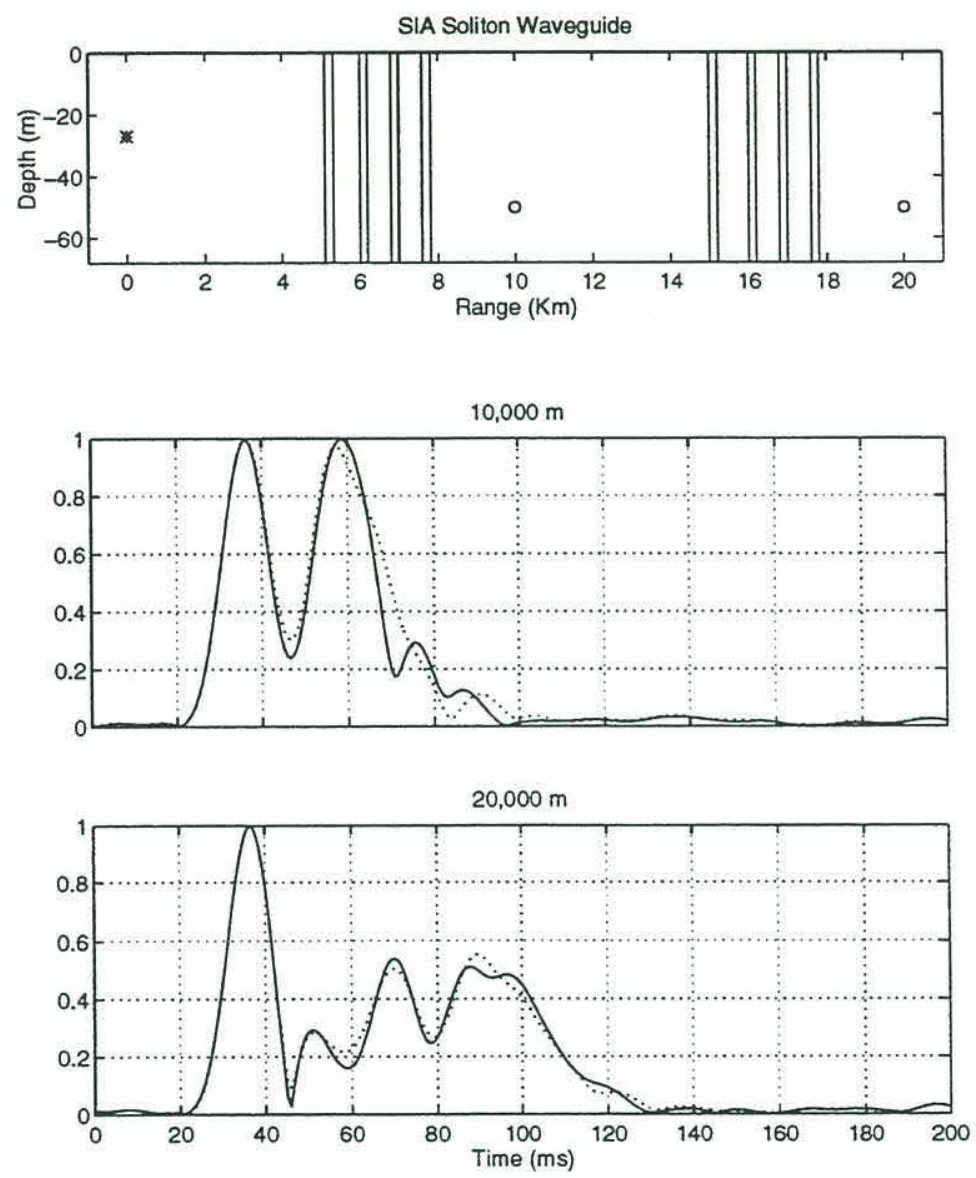

Figure B-2: An eight soliton waveguide (upper panel) and two corresponding arrivals computed from the PPM (solid) and KFS (dotted) methods. The modeled waveguide uses the SWARM SSPs shown in Figure 4-6 to simulate the solitons. The source $\left(^{*}\right)$ depth is $27 \mathrm{~m}$ and the receiver (o) depth at both ranges is $50 \mathrm{~m}$. 
Source Depth Effect on 10,000 m RIP
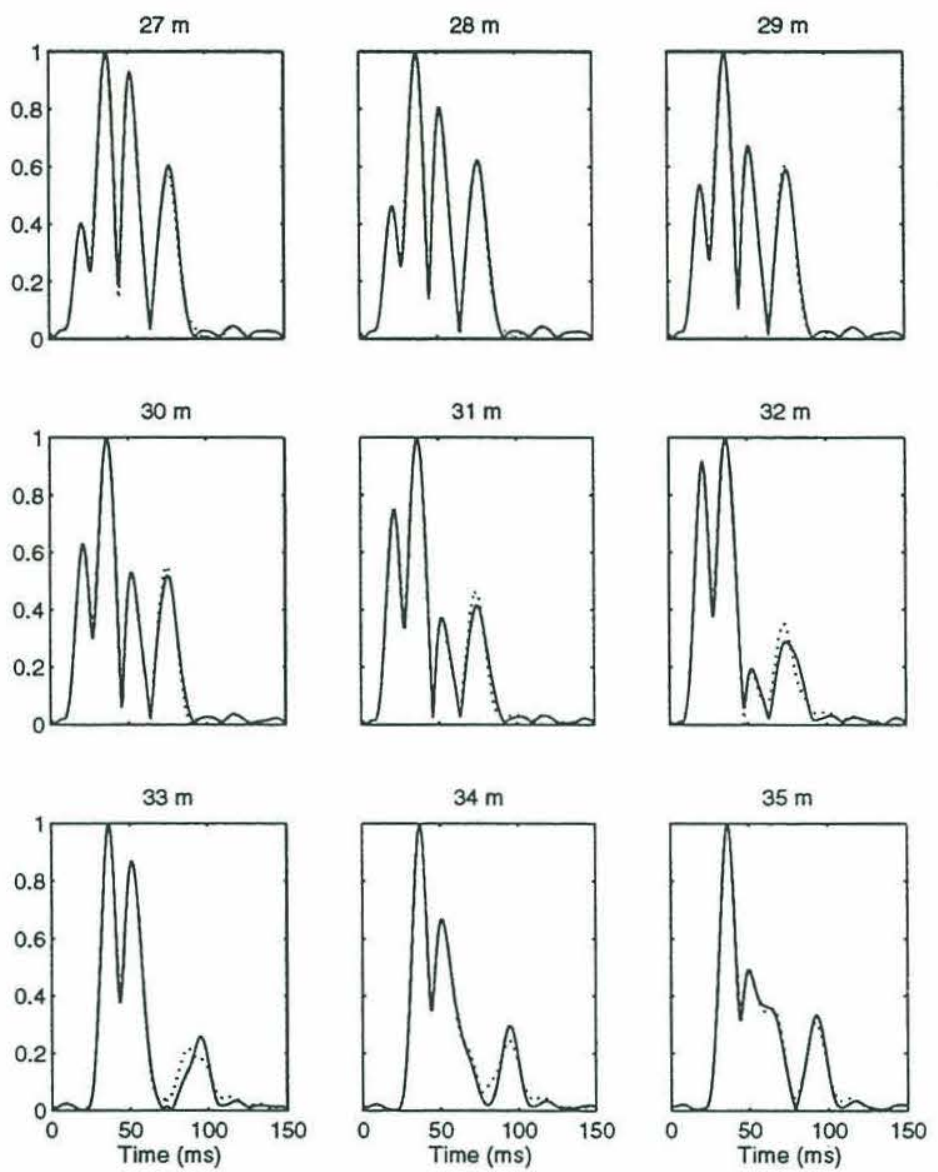

Figure B-3: Single hydrophone RIP pulse arrivals computed from the PPM (solid) and KFS (dotted) methods using various source depths with the receiver depth fixed at $50 \mathrm{~m}$.

for in the KFS method, but the PPM method assumes the $400 \mathrm{~Hz}$ coupling matrices apply at all frequencies. It is obviously not a major effect for one or two coupling events (see Figure B-1), but it may have cumulative effects over numerous coupling events.

The coupling matrices are not the only sources of deviation. Figure B-3 is evidence of the fact that deviations can exist between the two methods even in range independent propagation (RIP), where the aforementioned PPM narrowband coupling approximation is not a factor. The source depth dependence of the deviations 
in this figure point to the source excitation factor, $\phi_{4}\left(z_{s}\right)$, as a probable cause of the most significant deviations, but the modal wavenumber spectra and modal attenuation spectra used in the PPM cannot be ruled out as additional contributors to the deviations. 


\section{Appendix C}

\section{Incorporation of Background}

\section{Internal Wave Spectrum through Dozier and Tappert Coupling}

Our primary modeling concern in this thesis is internal wave solitons; their strong horizontal sound speed gradients cause significant scattering of energy between acoustic modes. Linear internal wave fields, which are also present in shallow water, cause additional scattering of energy between acoustic modes. They are usually modeled and studied as a stochastic process that produces random sound-speed fluctuations, $\delta c(r, z, t)$, about a background profile, $c_{0}(z)$. A "standard method" of producing these random sound speed fluctuations and resultant coupling matrices in deep water was developed by Dozier and Tappert (DT)[14]. We will follow their method here.

\section{C.1 Coupled Mode Formulation}

The DT method generally consists of using a GM spectrum as the basis for creating a random realization of the internal wavefield, $\zeta(r, z, t)$, which from Munk and 
Zachariasen [21] is proportional to $\delta c(r, z, t)$ through,

$$
\delta c(r, z, t) \propto \frac{N^{2}(z)}{g} \zeta(r, z, t),
$$

where $g$ is the gravitational acceleration and $N(z)$ is the Brunt-Vaisala (buoyancy) frequency. Dozier and Tappert [14] use the following normal mode formulation (with cylindrical spreading removed) for the pressure field,

$$
p(r, z, t)=e^{-i \omega t} \sum_{n=1}^{N} \xi_{n}(r, t)\left(k_{r n}\right)^{-1 / 2} \phi_{n}(z),
$$

where $\xi_{n}(r, t)=A_{n}(r, t)\left(k_{r n}\right)^{1 / 2}$ is a modified mode amplitude that yields a symmetric coupling matrix. Using the quasi-static and narrow angle forward scattering approximations, they developed the coupled mode equation

$$
\frac{\partial \xi_{n}}{\partial r}(r, t)-i k_{r n} \xi_{n}(r, t)=-i \sum_{m=1}^{N} R_{n m}(r, t) \xi_{m}(r, t),
$$

where the coupling matrix is

$$
R_{n m}(r, t)=\frac{\omega^{2}}{c_{0}^{2}\left(k_{r n} k_{r m}\right)^{1 / 2}} \int d z \frac{\delta c(r, z, t)}{c_{0}} \phi_{n}(z) \phi_{m}(z) .
$$

Creamer [18] closely follows Dozier and Tappert in his analysis of shallow-water waveguides; he retains the GM spectrum assumption and the basic form of the coupled mode equations, but he adds a bottom loss via the addition of $i \alpha_{n}$ to the horizontal wave number, $k_{r n}$, and introduces a simplification to the determination of $\delta c(r, z, t)$. Although the procedure is still complicated, he bypasses the actual determination of the internal wave field in favor of computing a $\delta c(r, z, t)$ field that is (1) zero-mean and gaussian and (2) has a correlation function consistent with that due to a GM internal wave spectrum.

It is unclear as to how closely a GM spectrum approximates shallow water inter- 
nal waves. For example, Lynch et al. [16] present a spectral analysis of data from a thermistor string in $400 \mathrm{~m}$ of water that is consistent with a GM spectrum at frequencies above about $8 \mathrm{cpd}$, but the spectrum in the 3 to $8 \mathrm{cpd}$ region is significantly less energetic relative to GM. This single string could not provide any information concerning the directional spectrum in the region.

Absent any other data to the contrary, the GM spectrum assumption provides a method of developing somewhat realistic levels of vertical and horizontal coherence. The vertical coherence is critical to the calculation of the coupling matrix at a given range and the horizontal coherence provides some realism to the range dependence of these matrices. Conversely, since the GM spectrum may not really be representative of shallow water, the modeling of correlation functions through a rather complicated GM internal wave synthesis may also not be the best approach.

\section{C.1.1 EOF Sound-speed Perturbation Modes}

Empirical orthogonal function (EOF) modes of thermistor string temperature perturbations are a good alternative to internal wave synthesis. Unlike the GM synthesis approximations, the EOF modes will include influence from both linear and nonlinear internal waves, so the EOF modes can be used as stand alone random realizations (i.e. no SIA solitons are required).

For small perturbations in temperature, the associated sound-speed fluctuations can be approximated by

$$
\delta c(r, z, t) \simeq(4.6-.11 T) \times \delta T(r, z, t)
$$

where $T$ is in degrees centigrade. With proper scaling, the EOF vertical mode shapes for the perturbations recorded by a vertical array of thermistors can be used to synthesize perturbations in sound speed as a function of depth. For a single realization

of sound-speed perturbations at a particular range, using an average temperature of 
18 degrees centigrade in the thermocline, we let

$$
\delta \hat{c}(r, z)=2.6 \sum_{n=1}^{N} \hat{B}_{n}(r) U_{v n}(z)
$$

where the $\hat{B}_{n}(r)$ are zero-mean gaussian random variables, and the $U_{v n}(z)$ are the vertical EOF modes obtained through a singular value decomposition of 12 days (over 17,000 samples) of data from an eleven thermistor string located between the source and receiver. The procedure determines 11 vertical modes, but only the first six are used in the numerical trials that follow.

The determination of horizontal correlations through the range dependence of $\hat{B}_{n}(r)$ is not as straight forward. EOF vertical mode coefficients, $b_{n}(t)$, are determined from modal analysis,

$$
b_{n}(t)=U_{v n}(z)^{-1} T(z, t),
$$

where $T(z, t)$ is the thermistor string record. The single time series $b_{n}(z, t)$ is then broken into 23 different time series that are one tidal cycle (12.42 hrs or 746 minutes) long. The transformation from time dependence to range dependence is made by assuming all the temperature disturbances are propagating without dispersion at 0.8 $\mathrm{m} / \mathrm{s}$ along the same line as the acoustic transmission path. This is reasonable for the nonlinear wave perturbations, as the majority observed during SWARM traveled in that direction. The solibore internal tide appears from thermistor records to also travel at a speed of around $.8 \mathrm{~m} / \mathrm{s}$. Propagation directions offset from the acoustic path would weight the average phase speed higher. For linear internal waves, at frequencies above about $3 \mathrm{cpd}$, phase speeds of around $.35 \mathrm{~m} / \mathrm{s}$ are appropriate. An isotropic directional spectrum for these linear internal waves yields a median phase speed of $.5 \mathrm{~m} / \mathrm{s}$ relative to the propagation path. The mean phase speed is infinite, but it lowers to $.8 \mathrm{~m} / \mathrm{s}$, if the minimum phase angle considered is set at .05 instead of 0 radians. The 0 radian limit is not reasonable if wavefront curvature is considered.

All things considered, $0.8 \mathrm{~m} / \mathrm{s}$ is reasonable estimate for an "all-wave average phase 
speed" relative to the propagation path. The resulting range dependent temperature profiles never existed in reality, but they are approximations of range dependent profiles that could reasonably exist. By this reasoning, each 746 minute set of mode coefficient data is assumed to represent $35.8 \mathrm{~km}$ in range through

$$
B_{n}(r)=b_{n}\left(t=\frac{r}{0.8 m / s}\right) .
$$

The 23 sets of vertical mode coefficients can then be analyzed for horizontal EOF modes, $U_{h m}(r)$. A single realization of $\hat{B}_{n}(r)$ is computed by

$$
\hat{B}_{n}(r)=\sum_{m=1}^{M} \beta_{m} U_{h m}(r)
$$

where the $\beta_{m}(r)$ are zero-mean gaussian random variables. This makes $\hat{B}_{n}(r)$ and $\delta \hat{c}(r, z)$ in turn zero-mean gaussian random variables. The 23 sets of data produce 22 horizontal EOF modes, but only the first 15 are used to synthesize sound-speed perturbation fields in the numerical trials that follow.

The standard deviations, $\sigma_{\delta c}$, of $\delta \hat{c}(r, z)$ are controlled by the standard deviations, $\sigma_{m n}$, of the $\beta_{m}$ for each $U_{h m}(r)$ of each $U_{v n}(z)$. These standard deviations are simply that statistic as obtained from the 23 EOF analysis outputs,

$$
\beta_{m}=U_{h m}(r)^{-1} B_{n}(r),
$$

of the 23 sets of horizontal data. The $\sigma_{m n}$ thus produced yield a model with a range and depth averaged $\left\langle\left(\delta c / c_{0}\right)^{2}>\right.$ of $4.4 \times 10^{-6}$. Creamer [18] considers from $10^{-6}$ to $10^{-5}$ to be typical values, and Tielbuerger et al. [9] report a value of $5 \times 10^{-7}$ for their model. 


\section{C.1.2 Numerical Trials}

Numerical trials with the Dozier and Tappert [14] coupled mode equations using EOF mode sound-speed perturbation fields will demonstrate their effects on modal propagation. The coupled mode equations (4.14) in matrix form are

$$
\frac{\partial \xi}{\partial r}=i\left[K_{N}-R(r)\right] \xi
$$

where $K_{N}=\operatorname{diag}\left[k_{1}, \ldots, k_{N}\right]$ [15]. This equation has a local solution that can be represented by

$$
\xi(r+\Delta r)=\xi(r) e^{i\left[K_{N}-R(r)\right] \Delta r} .
$$

The implementation of the above equation in discrete steps is obtained through diagonalization of the coupling matrix $\left[K_{N}-R(r)\right] \Delta r . R(r)$, which is calculated from (C.4), derives its range dependence from a realization of $\delta \hat{c}(r, z)$ computed by (C.6). The step size, $\Delta r$, used in these trials is $480 \mathrm{~m}$. This undersamples the fields actual perturbations in soundspeed, as did Dozier and Tappert's [15] $1 \mathrm{~km}$ step size, but the

idea is that the application of a $\delta c=\frac{1}{2}(\delta c(r)+\delta c(r+\Delta r)$ to a region $\Delta r$ is equivalent to using a $\langle\delta c\rangle$, spatially averaged over $\Delta r$, when the results are averaged over numerous trials.

\section{Propagation without Attenuation}

When there is no attenuation, Dozier and Tappert [14] have shown that these coupled mode equations conserve energy and tend towards an equal partition of energy among the modes involved. The assumption of no attenuation is not reasonable in our case, but running the model without attenuation provides data for comparison with Dozier and Tappert and demonstrates the coupling effectiveness of the EOF field. Figure C-1 shows that all the energy is initially transmitted in mode 1, and an equal partition of energy is achieved at about $120 \mathrm{~km}$. The rate at which the system tends towards equilibrium is controlled by the strength of the coupling. Figure 4-11 


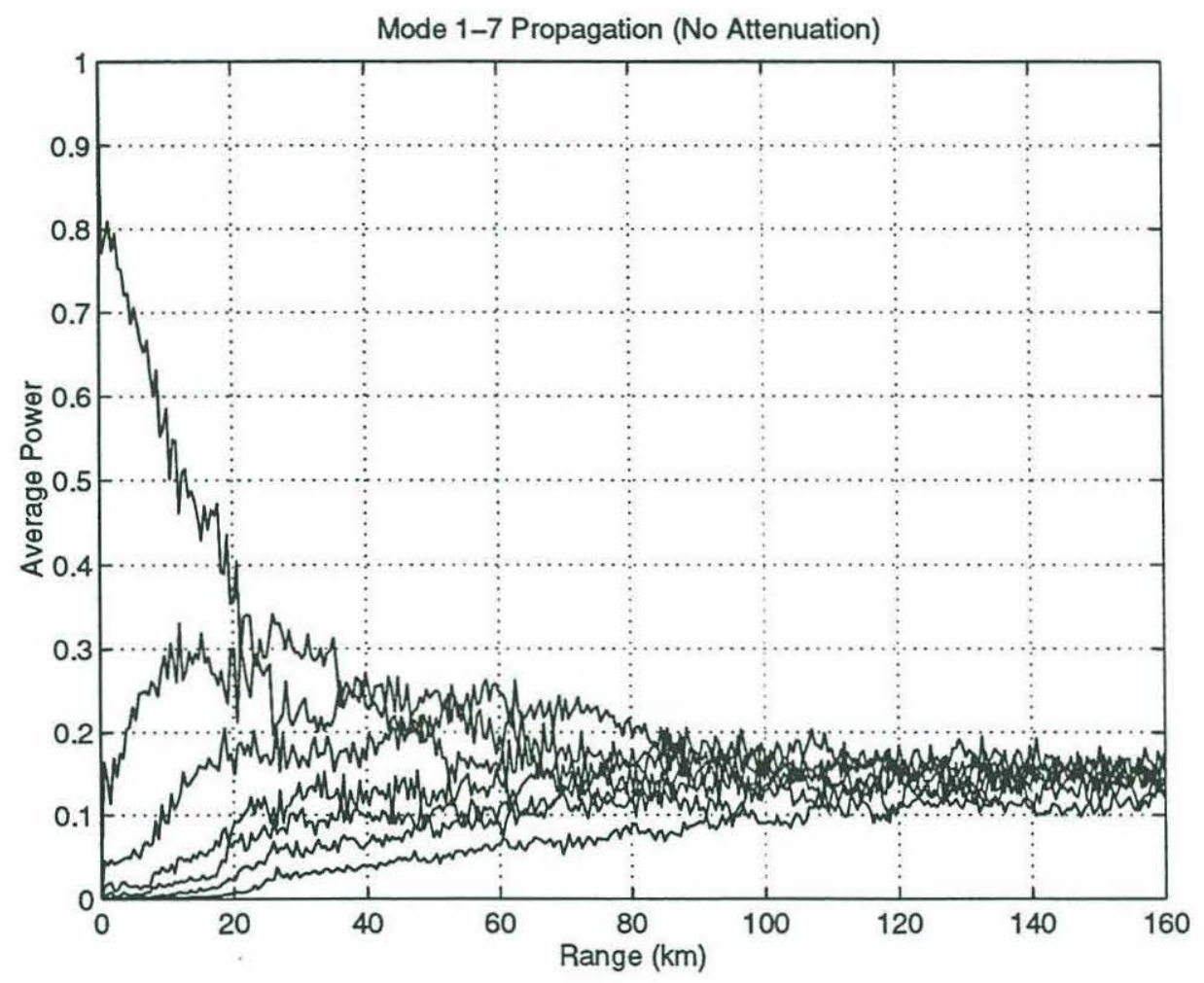

Figure C-1: Effects of linear internal wave scattering on mode propagation. The results shown are the average of 50 trials using a sound-speed perturbation model with a range and depth averaged $\left\langle\left(\delta c / c_{0}\right)^{2}>\right.$ of $1.4 \times 10^{-5}$. The mode shapes used in the coupling equations were from the standard field measured SSP at $400 \mathrm{~Hz}$. 
represents the average of 50 trials using random realizations of sound-speed perturbation fields produced by the EOF sound-speed model described above. The range and depth averaged $\left\langle\left(\delta c / c_{0}\right)^{2}\right\rangle$ was scaled up to $1.4 \times 10^{-5}$, speeding the approach to equilibrium. The field strength is easily scaled up or down. When scaled down it can be used in conjunction with SIA solitons, similar to a GM random realization, as a background field.

\section{Propagation with Attenuation}

When modal attenuation is added to the above coupling equations, Creamer [18] shows both analytically and numerically that in the limit of a large range, both the average modal intensity, $\langle I\rangle$, and fourth moment of pressure, $\left\langle I^{2}\right\rangle$, decay exponentially with range. The exponential decay rate of $\left\langle I^{2}\right\rangle$ is always less than or equal to the exponential decay rate of $\langle I\rangle^{2}$, because their difference, the variance of the intensity, must be greater than or equal to zero. Thus in the limit, the scintillation index, $S I$, defined as

$$
S I=\frac{\left\langle I^{2}\right\rangle}{<I\rangle^{2}}-1,
$$

grows exponentially in range. Repeating our numerical trials with modal attenuation applied reveals an asymptotic $S I+1$ exponential growth factor, $\chi$, of about 0.01 $\mathrm{km}^{-1}$ (Figure C-2). Creamer's [18] isovelocity model with average $\mathrm{GM}\left\langle\left(\delta c / c_{0}\right)^{2}\right\rangle$

perturbations of $10^{-5}$ yielded an $S I+1$ exponential growth rate of $0.02 \mathrm{~km}^{-1}$. The scattering ability of the EOF field clearly mimics that seen with GM fields, both with and without attenuation.

\section{C.2 Limitations of Dozier and Tappert Coupling}

Regardless of the form of internal wave field, the main limitation of any model that includes Dozier and Tappert [14] coupling is that the results are only valid in a stochastic sense. Both Dozier and Tappert [15] and Creamer [18] restrict their results 


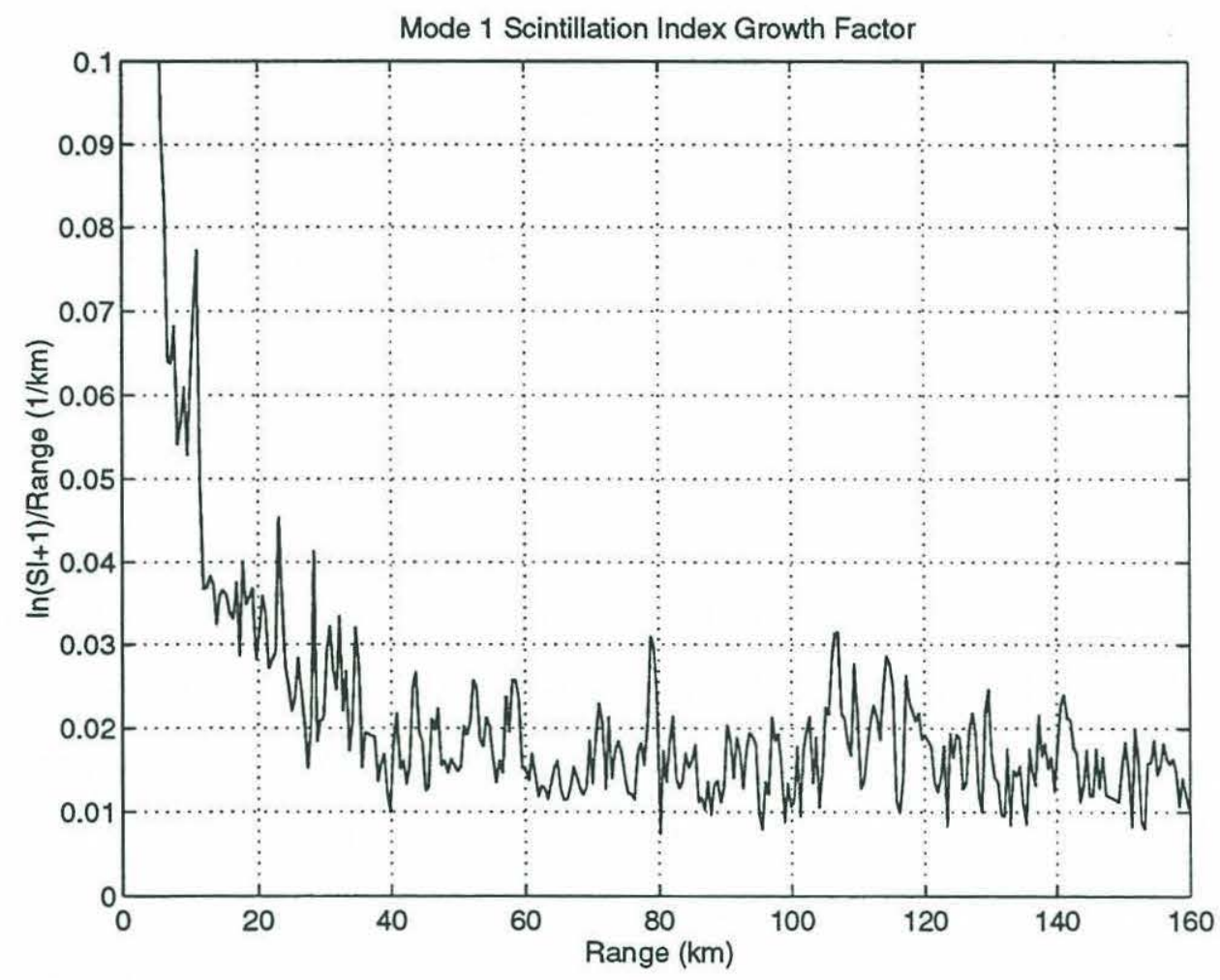

Figure C-2: Asymptotic behavior of $S I+1$ for mode 1 . The plotted quantity decreases to the asymptotic value of the growth rate factor $\chi$. The mode shapes and attenuation parameters used in the coupling equations were from the standard field measured SSP at $400 \mathrm{~Hz}$. 
to averages over a hundred or more random realizations.

\section{C.2.1 Fine Scale Application}

The large step size employed with Dozier and Tappert [14] coupling is the primary impediment to achieving a deterministic mode arrival model. If the implementation step size is reduced significantly, one conceivably moves from predicting the probability of energy transfer between modes over a large step to predicting actual levels of coupling over small steps, where $\delta c(r)$ at a point in the step is representative of $\delta c(r)$ over the entire step. The feasibility of this sort of fine scale application is evaluated using a test waveguide.

\section{Test Case}

In lieu of an arbitrary random realization of a GM or EOF internal wave field, we will attempt to apply these coupled-mode equations to waveguides derived directly from range propagated WVLA thermistor records. Using the arguments previously discussed for horizontal EOF analysis, the transformation from time dependence to range dependence is made by assuming all the temperature disturbances are propagating without dispersion at $0.8 \mathrm{~m} / \mathrm{s}$ along the same line as the acoustic transmission path.

Following the propagation step, the temperature records are interpolated in depth and converted to sound speed. The resulting range dependent sound speed profiles

$$
c(r, z, t)=c\left(z, t-\frac{r}{0.8 m / s}\right) .
$$

never existed in reality, but, again, they are approximations of range dependent profiles that might reasonably exist.

The next step involves subtracting a mean profile to create a sound speed pertur- 


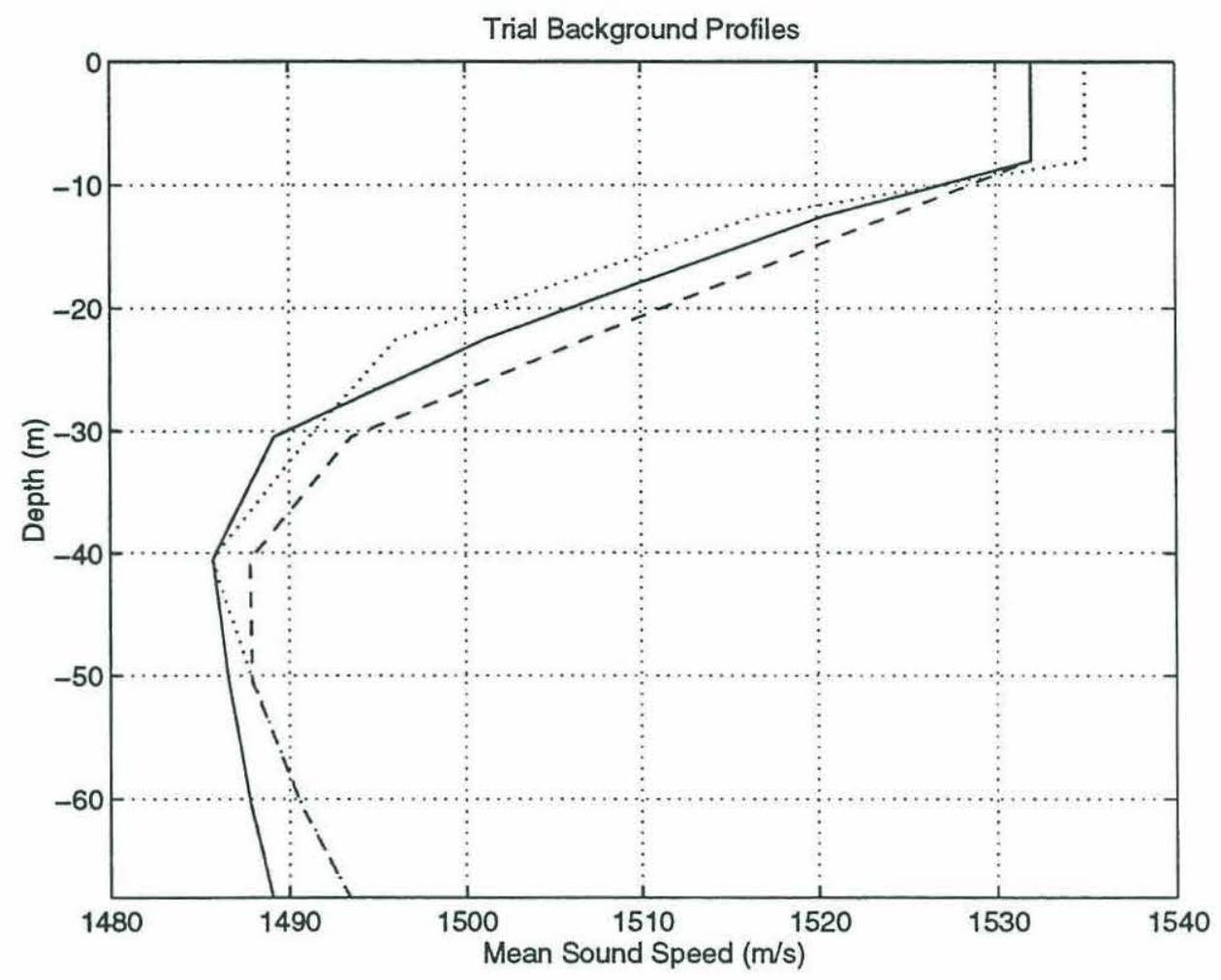

Figure C-3: Mean sound speed profiles obtained from the WVLA thermistor records. The solid line is an average over yearday 211-217, and the dashed line is averaged over yearday 211 only. The dotted SSP is taken from early in yearday 211 when no solitons were at the WVLA. All three are extrapolated for depths above $12.5 \mathrm{~m}$ and below $60.5 \mathrm{~m}$.

bation field,

$$
\delta c(r, z, t)=c(r, z, t)-c_{0}(z)
$$

Herein lies the difficulty, namely the choice of the mean profile to be used in computing the sound speed perturbation fields. Three possible choices, a one day average, a six day average, and an SSP chosen from a period without any solitons are shown in Figure C-3.

The choice of a mean profile determines the modeshapes, which, as shown in Figure C-4, affect the elements of the coupling matrices. Each mean profile also has slightly different dispersion curves; the phase and group speeds control the phasing between modes as a function of range, and the cumulative effect of the differences in dispersion 


\section{Coupling Coefficients (48 m increments)}
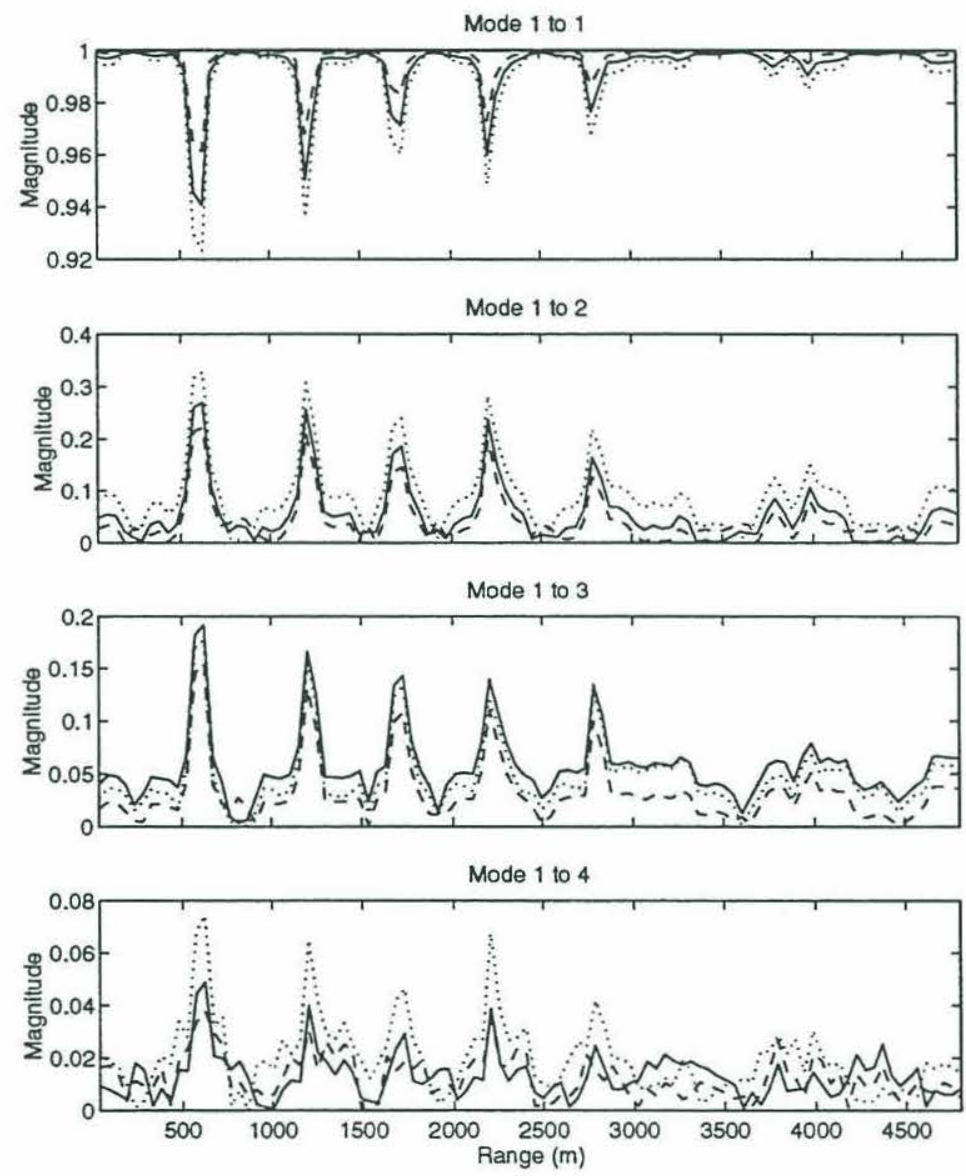

Figure C-4: Magnitudes of discrete coupling matrix coefficients using a $48 \mathrm{~m}$ step size with three different background profiles. The solid lines are computed using the yearday 211-217 mean profile, the dashed lines are from the yearday 211 mean profile, and the dotted lines are computed using the yearday 211 "no soliton" SSP. 


\section{Predicted Mode 1 Arrivals}
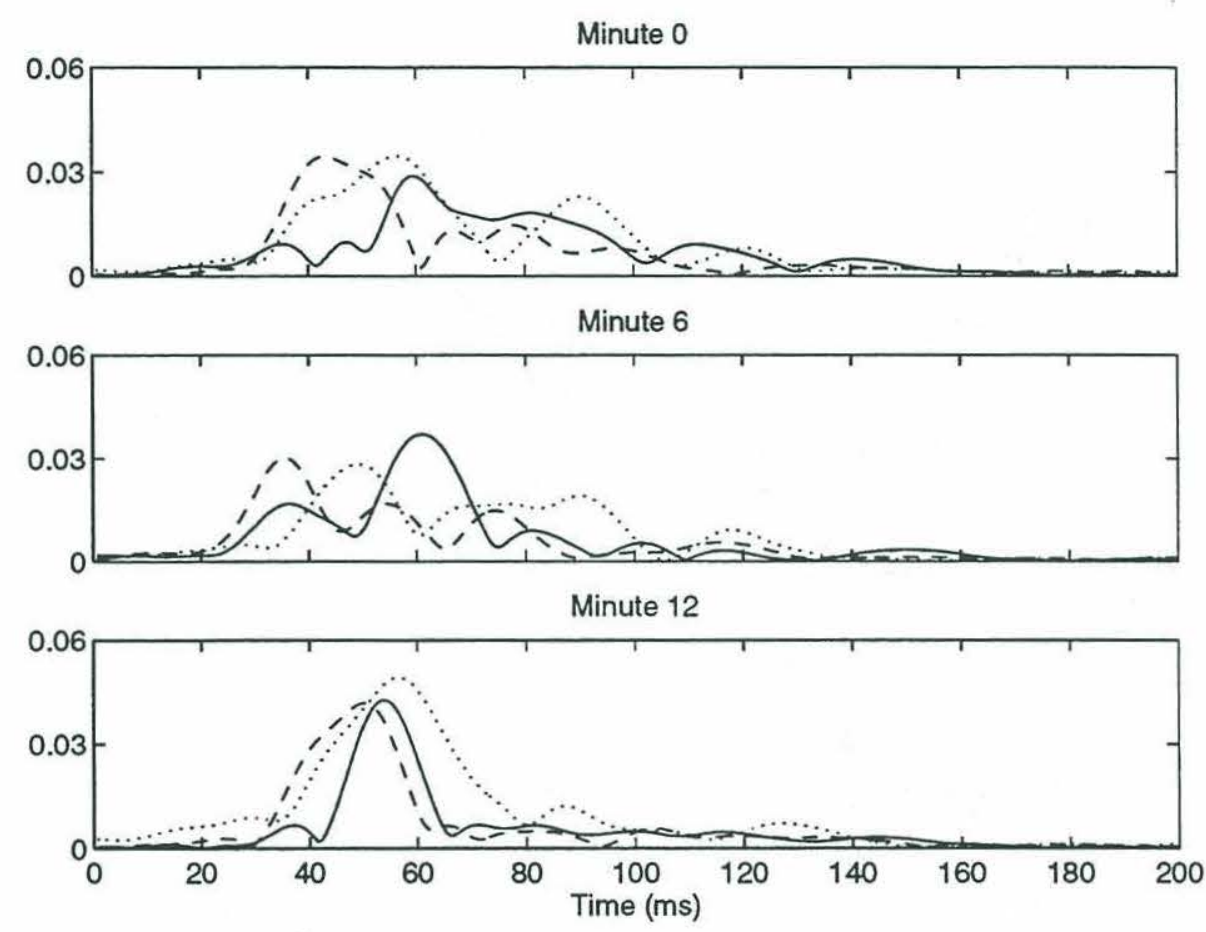

Figure C-5: Predictions of mode 1 arrivals made with three different background profiles. The solid lines are computed using the yearday 211-217 mean profile, the dashed lines are from the yearday 211 mean profile, and the dotted lines are computed using the yearday 211 "no soliton" SSP.

curves and coupling matrices can yield dissimilar outputs over long transmissions.

The outputs shown in Figure C-5 are from $32 \mathrm{~km}$ simulated transmissions that employ 670 propagation and coupling steps. These arrivals show dramatic differences between models for the minute 0 and minute 6 predictions, but the relatively unscattered minute 12 arrivals are surprisingly similar.

The statistical significance of the predicted arrivals using this method can be judged from Figure C-6, where 12 hours of arrivals are compared using two different mean profiles. Both show transitions from low to high scattering levels at about the same point in time, but the one day average mean, as might be expected, predicts less scattering in general. A near ideal (but impractical) choice for the background profile would be to use the range average of the soundspeed profiles used in each realization. 

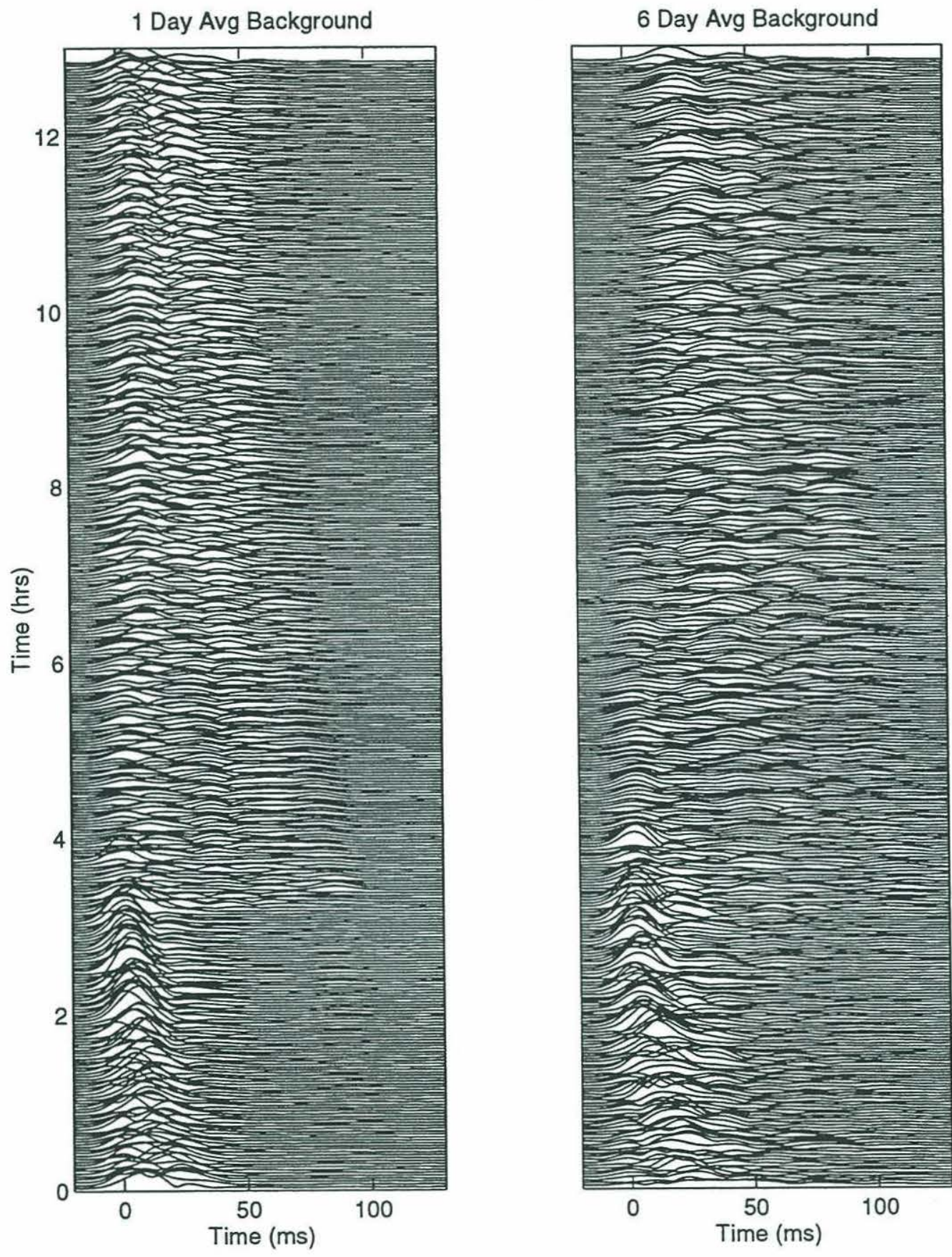

Figure C-6: Twelve hours of predicted mode 1 arrivals using the yearday 211 mean profile and the yearday 211-217 mean profile. 
This would amount to a series of $11 \mathrm{hr}$ averages and minimize the average magnitude of the sound speed perturbations for each transmission's waveguide. As it is, the yearday 211 mean profile yields a model with a $\left\langle\left(\delta c / c_{0}\right)^{2}>\right.$ of $6.4 \times 10^{-6}$, and the yearday 211-217 mean profile yields a model with a $\left\langle\left(\delta c / c_{0}\right)^{2}>\right.$ of $1.0 \times 10^{-5}$.

An argument can be made that in each case the soundspeed perturbations obtained from WVLA thermistor records are too large for the Dozier and Tappert coupled mode equations to be applicable. Clearly, as both long and short term fluctuations in sound speed decrease, the choice of the background profile becomes more deterministic, and the "mean profile" uncertainties on predicted mode 1 arrivals are diminished. Dozier and Tappert's [14] deep water model has sound speed perturbations that are at least an order of magnitude less. They have a maximum range averaged $<\left(\delta c / c_{0}\right)^{2}>$ of about $2.5 \times 10^{-7}$, and the perturbations decay exponentially in depth. Their model has no uncertainty in the mean profile, as it is assumed from the outset. Similarly, Creamer [18], who uses the Dozier and Tappert coupling equations in his shallow water model, has no uncertainty associated with his isovelocity mean profile, but his depth and range averaged $\left\langle\left(\delta c / c_{0}\right)^{2}\right\rangle$ values $10^{-6}$ and $10^{-5}$ are closer to the perturbations employed in our model.

Given the size of our soundspeed perturbations and the uncertainties associated with the background profile, we cannot expect to accurately predict a particular mode 1 arrival using equation (C.4). Therefore, regardless of the step size used, the predicted mode 1 arrival structures generated with Dozier and Tappert [14] coupling (Figure C-5 and C-6) are only significant in a statistical sense. 


\section{Appendix D}

\section{Modal Reciprocity}

The simulations discussed in Chapter 5 illustrate the sensitivity of mode spread and bias statistics to the position of solitons. Figures 5-9, 5-10, and 5-11, in particular, show how the movement of two soliton trains (away from the receiver and towards the source) reduces the potential spread in mode arrival envelopes.

This result predicts significant differences between the mode arrivals for reciprocal transmissions when the distribution of scattering regions between the transducers is highly asymmetric. In a test of this concept, a highly asymmetric SIA soliton waveguide (from Figure 5-9), is used for simulated reciprocal transmissions. The results of this test are shown in Figure D-1. The mode arrivals clearly show the expected decreases in modal spread for the reciprocal transmission, so there is no reciprocity in the individual mode arrivals. However, when the mode arrival predictions are synthesized to yield time domain pulses at the individual transducers,

$$
p(32 k m, 27 m, t)=\sum_{n} \tilde{p}_{n}(32 k m, t) \phi_{n}(27 m)
$$

(where the $\tilde{p}_{n}(r, t)$ are the mode arrivals and the $\phi(z)$ are the mode shapes), reciprocity between the source and receiver is revealed.

A somewhat simpler scenario is illustrated in Figure D-2. In this case a front, 

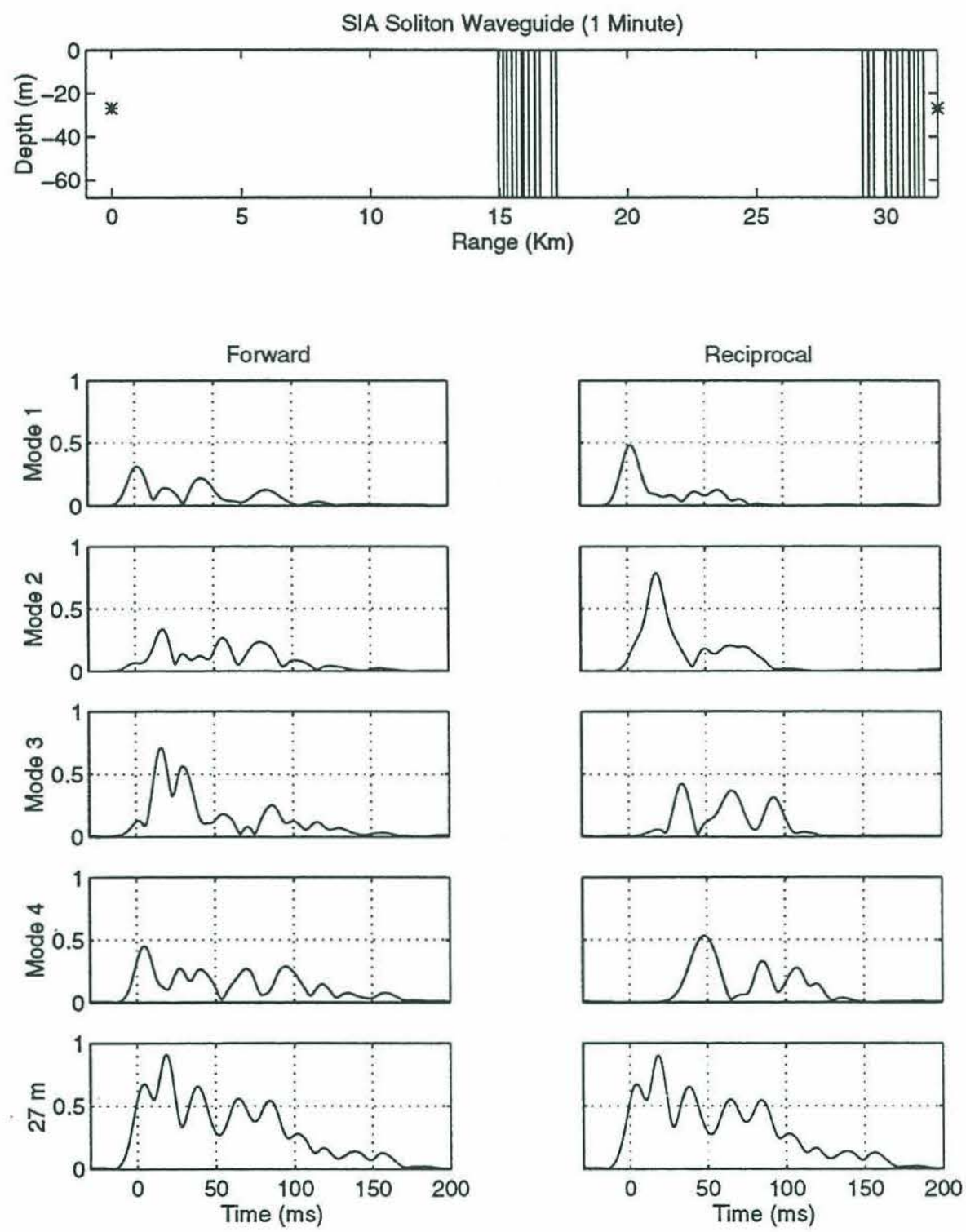

Figure D-1: A comparison of reciprocal transmissions through an asymmetric SIA soliton waveguide (upper frame). Mode 1 through 4 arrivals as well the time domain pulse at a single transducer $(27 \mathrm{~m})$ are shown for both the forward and reciprocal transmissions. The source/receiver depth is $27 \mathrm{~m}$ for both transducers. 

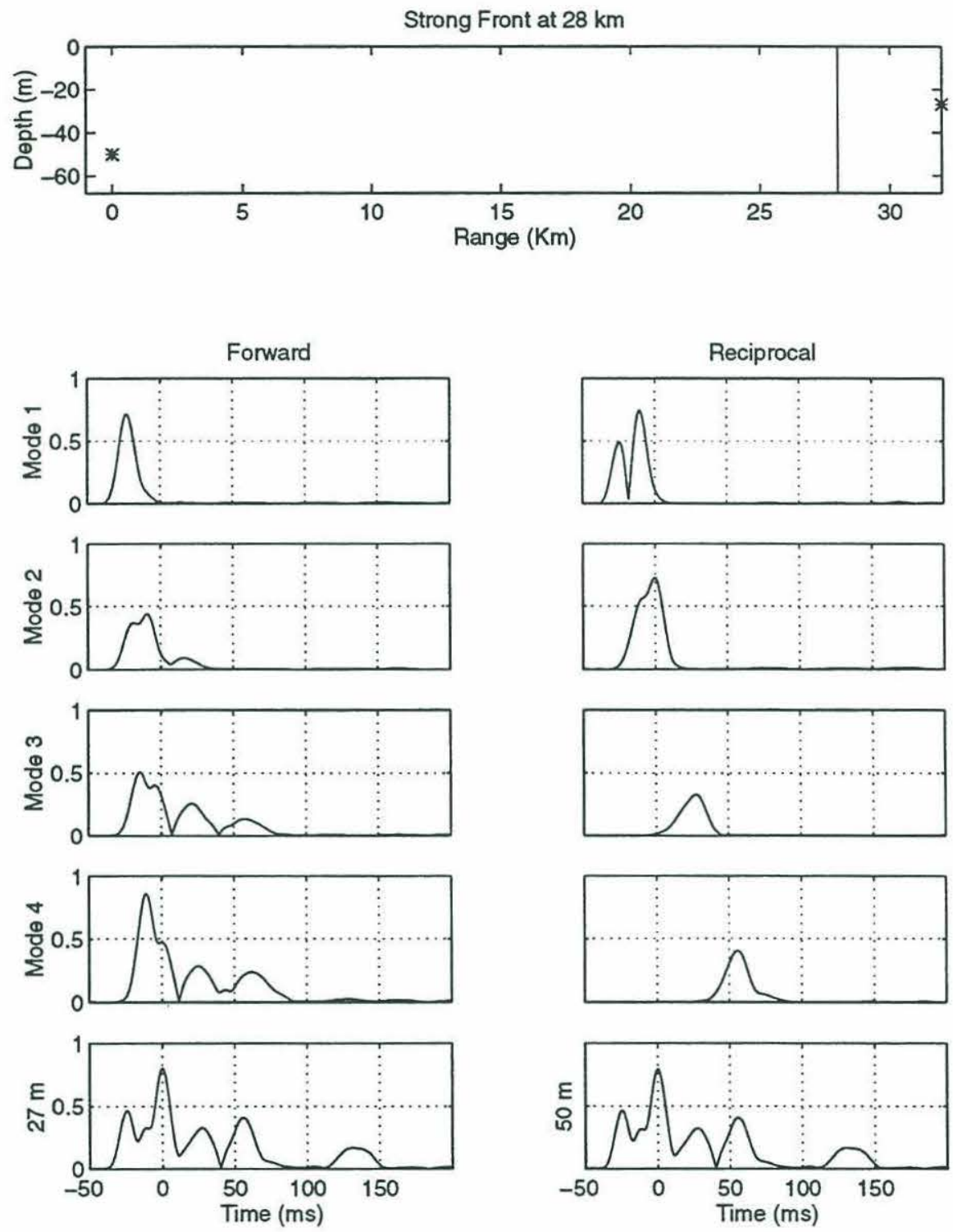

Figure D-2: A comparison of reciprocal transmissions through a strong front (upper frame). Mode 1 through 4 arrivals as well the time domain pulse at the receiver are shown for both the forward and reciprocal transmissions. The source/receiver depth is $50 \mathrm{~m}$ at $0 \mathrm{~km}$ and $27 \mathrm{~m}$ at $32 \mathrm{~km}$. 
requiring a single coupling event, is simulated at the $28 \mathrm{~km}$ point of $32 \mathrm{~km}$ waveguide. The standard "non-soliton" SSP is used from 0 to $28 \mathrm{~km}$ and the standard "soliton" SSP is used from 28 to $32 \mathrm{~km}$. Again there are significant differences in the individual mode arrivals, but the sums at the transducers show the reciprocity between the source and receiver. 


\section{Bibliography}

[1] The SWARM Group (J. R. Apel et al.). An overview of the 1995 swarm shallow water internal wave acoustic scattering experiment. IEEE JOE, accepted for publication, 1997.

[2] J. Preisig and T. F. Duda. Coupled acoustic mode propagation through continental shelf internal solitary waves. IEEE JOE, in press, 1997.

[3] C. Garrett and W. H. Munk. Space-time scales of internal waves. Geophys. Fluid Dyn., 2:225-264, 1972.

[4] G. Strang. Introduction to Applied Mathematics. Wellesley-Cambridge Press, Wellesley, Mass., 1986.

[5] J. R. Apel, L. A. Ostrovsky, and Y. A. Stepanyants. Internal solitons in the ocean. Technical Report MERCJRA0695, John Hopkins APL, July 1995.

[6] J. X. Zhou, X. Z. Zhang, and P. H. Rogers. Resonant interaction of sound wave with internal solitons in the coastal ocean. J. Acoust. Soc. Am., 90:2042-2054, 1991.

[7] J. X. Zhou, X. Z. Zhang, P. H. Rogers, D. Wang, and E. Luo. Anomalous sound propagation in shallow water due to internal wave solitons. I.E.E.E. J. of Oc. Sc., 90:2042-2054, 1993.

[8] J. X. Zhou and X. Z. Zhang. Modal sensitivities of acoustic interactions in shallow water. J. Acoust. Soc. Am., 98:2868, 1995. 
[9] D. Tielbuerger, S. Finette, and S. Wolf. Acoustic propagation through an internal wave field in a shallow water waveguide. J. Acoust. Soc. Am., 101:789-808, 1997.

[10] J. A. Colosi and S. M. Flatte. Mode coupling by internal waves for multimegameter acoustic propagation in the ocean. J. Acoust. Soc. Am., 100:3607-3620, 1996.

[11] A. V. Oppenheim and R. W. Schafer. Discrete-Time Signal Processing. PrenticeHall, Englewood Cliffs, N.J., 1989.

[12] M. B. Porter. The kraken normal mode program. Technical report, SACLANT Undersea Research Center, November 1992.

[13] F. B. Jensen, W. A. Kuperman, M. B. Porter, and H. Schmidt. Computational Ocean Acoustics. American Institute of Physics, New York N. Y., 1994.

[14] L. B. Dozier and F. D. Tappert. Statistics of normal mode amplitudes in a random ocean. i. theory. J. Acoust. Soc. Am., 63:353-365, 1978.

[15] L. B. Dozier and F. D. Tappert. Statistics of normal mode amplitudes in a random ocean. ii. computations. J. Acoust. Soc. Am., 64:533-547, 1978.

[16] J. F. Lynch, G. Jin, R. Pawlowicz, C.-S. Chiu, J. H. Miller, R. H. Bourke, A. R. Parsons, A. J. Plueddemann, and R. Muench. Acoustic travel time perturbations due to shallow water internal waves and internal tides in the barents sea polar front: theory and experiment. J. Acoust. Soc. Am., 99:803-821, 1996.

[17] W. A. Kuperman, M. B. Porter, J. S. Perkins, and R. B. Evans. Rapid computation of acoustic fields in three dimensional ocean environments. J. Acoust. Soc. Am., 89:125-133, 1991.

[18] D. B. Creamer. Scintillating shallow-water waveguides. J. Acoust. Soc. Am., 99:2825-2838, 1996. 
[19] A. W. Drake. Fundamentals of Applied Probability Theory. McGraw-Hill Book Company, New York, N. Y., 1967.

[20] M. S. Triantafyllou. Cable mechanics with marine applications. Technical report, MIT, May 1990.

[21] W. H. Munk and F. Zachariasen. Sound propagation through a fluctuating stratified ocean: Theory and observation. J. Acoust. Soc. Am., 59:818-838, 1976. 